\title{
GEOMETRIC EISENSTEIN SERIES
}

\author{
A. BRAVERMAN AND D. GAITSGORY
}

\section{Contents}

Introduction

0.1. Motivation

0.2. Survey of the main results

0.3. Contents

0.4. Conventions

1. Definition of Drinfeld's compactifications

1.1. Strongly quasi-affine varieties

1.2. Drinfeld structures

\begin{tabular}{lll}
\hline 1.3. & Parabolic Drinfeld's structures & 14
\end{tabular}

\begin{tabular}{lll}
\hline 2. & Main results on Eisenstein series & 17
\end{tabular}

\begin{tabular}{ll}
2.1. & Principal Eisenstein series \\
\hline
\end{tabular}

$\begin{array}{lll}2.2 . & \text { Relation with the classical theory } & 21\end{array}$

\begin{tabular}{lll}
\hline 2.3. & Non-principal Eisenstein series & 25
\end{tabular}

\begin{tabular}{|lll}
\hline $3 . \quad$ Action of Hecke operators (the principal case) & 27
\end{tabular}

$\begin{array}{lll}3.1 . & \text { The basic diagram } & 27\end{array}$

$\begin{array}{lll}3.2 . & \text { Preliminaries on the affine Grassmannian } & 29\end{array}$

3.3. Proof of Theorem 3.1.4 33

4. Action of Hecke operators (the non-principal case) 36

$\begin{array}{lll}4.1 . & \text { Variant of the basic diagram } & 36\end{array}$

\begin{tabular}{lll}
\hline 4.2. & Proof of Theorem 4.1.3 & 39
\end{tabular}

4.3. Proof of Theorem 4.1.5 43

\begin{tabular}{ll|l}
\hline 5. & The acyclicity theorem & 47
\end{tabular}

$\begin{array}{lll}5.1 . & \text { The notion of local acyclicity } & 47\end{array}$

\begin{tabular}{lll}
5.2. & Proof of Theorem 5.1.5 & 49 \\
\hline 5.3. & in the Borel case & 49
\end{tabular}

$\begin{array}{lll}5.3 . & \text { Proof of Theorem 5.1.5 in the general case } & 51\end{array}$

\begin{tabular}{lll}
\hline 6. & The structure of Drinfeld's compactifications & 54
\end{tabular}

\begin{tabular}{lll}
\hline 6.1. & Stratifications-1 & 54
\end{tabular}

\begin{tabular}{lll}
\hline 6.2. & Stratifications-II & 56
\end{tabular}

$\begin{array}{lll}\text { 6.3. } & \text { Proof of Theorem 6.2.10 } & 60\end{array}$

6.4. "Good" perverse sheaves and Theorem 2.3.4 63

$\begin{array}{lll}7 . & \text { The Functional equation } & 65\end{array}$

$\begin{array}{lll}7 \text { 7.1. } & \text { Theorem 7.1.6 } & 65\end{array}$

\begin{tabular}{lll}
\hline 7.2. & Composing Eisenstein series & 68
\end{tabular}

\begin{tabular}{lll}
\hline 7.3. & Computation in rank 1 & 70
\end{tabular}

\begin{tabular}{ll}
\hline Appendix & 75
\end{tabular} 


\section{INTRODUCTION}

\subsection{Motivation.}

0.1.1. Let $X$ be a curve over $\mathbb{F}_{q}$ and let $G$ be a reductive group. The classical theory of automorphic forms is concerned with the space of functions on the quotient $G_{\mathbb{A}} / G_{\mathcal{K}}$, where $\mathcal{K}$ (resp., $\mathbb{A}$ ) is the field or rational functions on $X$ (resp., the ring of adèles of $\mathcal{K})$. In this paper, we will consider only the unramified situation, i.e. we will study functions (and afterwards perverse sheaves) on the double quotient $G_{\mathbb{O}} \backslash G_{\mathbb{A}} / G_{\mathcal{K}}$.

Let $T$ be a Cartan subgroup of $G$. There is a well-known construction, called the Eisenstein series operator that attaches to a compactly supported function on $T_{\mathbb{O}} \backslash T_{\mathbb{A}} / T_{\mathcal{K}}$ a function on $G_{\mathbb{O}} \backslash G_{\mathbb{A}} / G_{\mathcal{K}}$ :

Consider the diagram

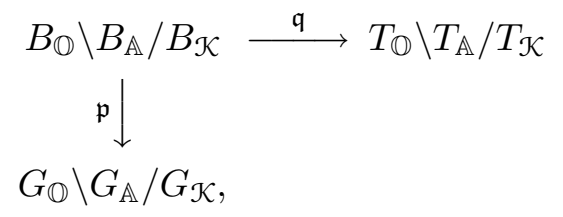

where $B$ is a Borel subgroup of $G$. Up to a normalization factor, the Eisenstein series of a function $S$ on $T_{\mathbb{O}} \backslash T_{\mathbb{A}} / T_{\mathcal{K}}$ is $\mathfrak{p}_{!}\left(\mathfrak{q}^{*}(S)\right)$, where $\mathfrak{q}^{*}$ denotes pull-back and $\mathfrak{p}_{\text {! }}$ is integration along the fiber.

Our goal is to study a geometric analog of this construction.

0.1.2. Let $\operatorname{Bun}_{G}$ denote the stack of $G$-bundles on $X$. One may regard the derived category of constructible sheaves on $\operatorname{Bun}_{G}$ (denoted $\operatorname{Sh}\left(\operatorname{Bun}_{G}\right)$, cf. Sect. D.4) as a geometric analog of the space of functions on $G_{\mathbb{O}} \backslash G_{\mathbb{A}} / G_{\mathcal{K}}$. Then, by geometrizing the Eisenstein series operator, we obtain an Eisenstein series functor $\operatorname{Eis}^{\prime}: \operatorname{Sh}\left(\operatorname{Bun}_{T}\right) \rightarrow$ $\mathrm{Sh}\left(\operatorname{Bun}_{G}\right)$ defined by a diagram similar to the above one, where the intermediate stack is Bun $_{B}$-the stack of $B$-bundles on $X$.

However, this construction has an immediate drawback-it is not sufficienly functorial (for example it does not commute with Verdier duality), the reason being that the projection $\mathfrak{p}: \operatorname{Bun}_{B} \rightarrow \operatorname{Bun}_{G}$ has non-compact fibers. Therefore, it is natural to look for a relative compactification of $\operatorname{Bun}_{B}$ along the fibers of the projection $\mathfrak{p}$.

Such a compactification, denoted $\overline{\mathrm{Bun}}_{B}$, was found by V. Drinfeld. We will use it to define the corrected functor Eis $: \operatorname{Sh}\left(\operatorname{Bun}_{T}\right) \rightarrow \operatorname{Sh}\left(\operatorname{Bun}_{G}\right)$. This paper is devoted to the investigation of various properties of this functor.

As we will see, all the technical results will essentially reduce to questions about the geometry of $\overline{\mathrm{Bun}}_{B}$ and the behaviour of the intersection cohomology sheaf on it.

0.1.3. We should say right away that the pioneering work in this direction was done by G. Laumon in [19], who considered the case of $G=G L(n)$ using his own compactification of the stack $\operatorname{Bun}_{B}$. In the sequel we will explain how the two approaches are related. 


\section{2 . Survey of the main results.}

0.2.1. First, let us indicate how Drinfeld's compactifications are constructed.

Let $G=G L(2)$. In this case, the stack $\operatorname{Bun}_{B}$ classifies pairs $(\mathcal{M}, \mathcal{L}, \kappa: \mathcal{L} \hookrightarrow \mathcal{M})$, where $\mathcal{M}$ is a rank 2 vector bundle on $X, \mathcal{L}$ is a line bundle on $X$, and $\kappa$ is a nonzero (=injective) bundle map. When $\mathcal{M}$ is fixed, the set of all possible $(\mathcal{L}, \kappa)$ forms an algebraic variety. This variety is non-complete, but there is a natural way to compactify it:

The corresponding complete variety also consists of pairs $(\mathcal{L}, \kappa: \mathcal{L} \rightarrow \mathcal{M})$, but this time $\kappa$ is required to be just an injective map of coherent sheaves, and not necessarily a bundle map. In other words, the quotient $\mathcal{M} / \mathcal{L}$ may have torsion. By definition, the stack $\overline{\operatorname{Bun}}_{B}$ for $G L(2)$ classifies triples $(\mathcal{M}, \mathcal{L}, \kappa)$, where $\kappa$ is understood in the new sense.

For a general $G$ (we are assuming that the derived group of $G$ is simply-connected) the construction of $\overline{\mathrm{Bun}}_{B}$ is very similar.

Let $\mathcal{F}_{G}$ be a fixed $G$-bundle. Let us denote by $\mathcal{V}_{\mathcal{F}_{G}}^{\check{\lambda}}$ the vector bundle on $X$ associated with $\mathcal{F}_{G}$ and a highest weight $G$-module $\mathcal{V}^{\check{\lambda}}$. A point of $\operatorname{Bun}_{B}$ that projects to our $\mathcal{F}_{G} \in \operatorname{Bun}_{G}$ defines in every $\mathcal{V}_{\mathcal{F}_{G}}^{\check{\lambda}}$ as above a line subbundle $\mathcal{L}^{\check{\lambda}}$, such that the system of these subbundles satisfies the so-called Plücker relations, cf. Sect. 1.

By definition, $\overline{\operatorname{Bun}}_{B}$ classifies the data of $\mathcal{F}_{G}$ endowed with a system of maps $\kappa^{\check{\lambda}}$ : $\mathcal{L}^{\check{\lambda}} \hookrightarrow \mathcal{V}_{\mathcal{F}_{G}}^{\check{\lambda}}$ defined for every dominant integral weight $\check{\lambda}$, where $\kappa^{\check{\lambda}}$ are injective as maps of coherent sheaves, which satisfy the Plücker relations.

Along the same lines one can try to define a compactification of the stack $\operatorname{Bun}_{P}$, where $P$ is a parabolic subgroup of $G$. However, in this case there will be two different natural compactifications $\overline{\operatorname{Bun}}_{P}$ and $\widetilde{\operatorname{Bun}}_{P}$, of which the second one will be more important for our purposes.

Let us make a brief digression and discuss Laumon's compactification of Bun for $_{B}$ $G=G L(n)$. In this case, $\operatorname{Bun}_{B}$ is the stack classifying the data of a rank $n$ vector bundle $\mathcal{M}$ on $X$ endowed with a complete flag $0=\mathcal{M}_{0} \subset \mathcal{M}_{1} \subset \ldots \subset \mathcal{M}_{n}=\mathcal{M}$ of subbundles. Laumon's compactification $\overline{\operatorname{Bun}}_{B}^{L}$ classifies the data of $(\mathcal{M}+$ a flag of $\mathcal{M}_{i}$ 's), but each $\mathcal{M}_{i}$ is required to be just a subsheaf (rather than a subbundle) of the subsequent $\mathcal{M}_{i+1}$.

For $G L(2)$, Laumon's and Drinfeld's compactifications coincide, but this is no longer true for $n \geq 3$. The advantage of Laumon's compactification is that $\overline{\operatorname{Bun}}_{B}^{L}$ is smooth, whereas $\overline{\operatorname{Bun}}_{B}$ is singular for $G$ of semi-simple rank $>1$. The drawback is that $\overline{\operatorname{Bun}}_{B}^{L}$ makes sense only for $G L(n)$.

In fact, there is a natural (proper and representable) map of stacks $\overline{\operatorname{Bun}}_{B}^{L} \rightarrow \overline{\operatorname{Bun}}_{B}$ and it was shown by A. Kuznetsov in 18 that $\overline{\operatorname{Bun}}_{B}^{L}$ is a small resolution of singularities of $\overline{\mathrm{Bun}}_{B}$.

0.2.2. Once the stack $\overline{\operatorname{Bun}}_{B}$ is constructed, one can try to use it to define the "compactified" Eisenstein series functor Eis : $\operatorname{Sh}\left(\operatorname{Bun}_{T}\right) \rightarrow \operatorname{Sh}\left(\operatorname{Bun}_{G}\right)$. Let $\overline{\mathfrak{p}}$ and $\overline{\mathfrak{q}}$ denote the natural projections from $\overline{\mathrm{Bun}}_{B}$ to $\mathrm{Bun}_{G}$ and $\mathrm{Bun}_{T}$, respectively. The first idea would be to consider the functor $\mathcal{S} \in \operatorname{Sh}\left(\operatorname{Bun}_{T}\right) \mapsto \overline{\mathfrak{p}}_{!}\left(\overline{\mathfrak{q}}^{*}(\mathcal{S})\right) \in \operatorname{Bun}_{G}$. However, this is 
too naive, since if we want our functor to commute with Verdier duality, we need to take into account the sigularities of $\overline{\operatorname{Bun}}_{B}$. Therefore, one introduces a kernel on $\overline{\operatorname{Bun}}_{B}$ given by its intersection cohomology sheaf. I.e., we define the functor Eis by

$$
\mathcal{S} \mapsto \overline{\mathfrak{p}}_{!}\left(\overline{\mathfrak{q}}^{*}(\mathcal{S}) \otimes \mathrm{IC}_{\overline{\mathrm{Bun}}_{B}}\right),
$$

up to a cohomological shift and Tate's twist. Similarly, one defines the functor $\operatorname{Eis}_{M}^{G}$ : $\operatorname{Sh}\left(\operatorname{Bun}_{M}\right) \rightarrow \operatorname{Sh}\left(\operatorname{Bun}_{G}\right)$, where $M$ is the Levi quotient of a parabolic $P$.

The first test whether our definition of the functor Eis is "the right one" would be the assertion that Eis (or more generally $\operatorname{Eis}_{M}^{G}$ ) indeed commutes with Verdier duality. Our Eis passes this test, according to Corollary 2.1.3.

Let us again add a comment of how the functor Eis is conneceted to Laumon's work. One can define functors $\operatorname{Eis}^{L}: \operatorname{Sh}\left(\operatorname{Bun}_{T}\right) \rightarrow \operatorname{Sh}\left(\operatorname{Bun}_{G}\right)$ using Laumon's compactification. (In the original work [19], Laumon did not consider $\operatorname{Eis}^{L}$ as a functor, but rather applied it to specific sheaves on $\operatorname{Bun}_{T}$.) However, from the smallness result of [18] it follows that the functors $\mathrm{Eis}^{L}$ and Eis are canonically isomorphic.

The situation is more subtle when one considers non-principal Eisenstein series, i.e. when $B$ is replaced by a more general parablic. In this case, it is no longer true that the two definitions agree. (In particular, for $P \neq B$, Laumon's Eisenstein series fails to commute with Hecke functors, cf. below.) At the end of Sect. 2 we suggest a conjecture of how they may be related.

0.2.3. Once we defined the functors $\operatorname{Eis}=\operatorname{Eis}_{T}^{G}: \operatorname{Sh}\left(\operatorname{Bun}_{T}\right) \rightarrow \operatorname{Sh}\left(\operatorname{Bun}_{G}\right), \operatorname{Eis}_{M}^{G}$ : $\operatorname{Sh}\left(\operatorname{Bun}_{M}\right) \rightarrow \operatorname{Sh}\left(\operatorname{Bun}_{G}\right)$ and a similar functor for $M, \operatorname{Eis}_{T}^{M}: \operatorname{Sh}\left(\operatorname{Bun}_{T}\right) \rightarrow \operatorname{Sh}\left(\operatorname{Bun}_{M}\right)$, it is by all means natural to expect that these functors compose nicely, i.e. that $\operatorname{Eis}_{T}^{G} \simeq \operatorname{Eis}_{M}^{G} \circ \operatorname{Eis}_{T}^{M}$.

For example, if instead of Eis we used the naive (uncompactified) functor Eis', the analogous assertion would be a triviality, since $\operatorname{Bun}_{B} \simeq \operatorname{Bun}_{P} \underset{\operatorname{Bun}_{M}}{\times} \operatorname{Bun}_{B(M)}$, where $B(M)$ is the Borel subgroup of $M$.

The problem with our definition of $\operatorname{Eis}_{M}^{G}$ is that there is no map between the relevant compactifications, i.e. from $\overline{\operatorname{Bun}}_{B}$ to $\widehat{\operatorname{Bun}}_{P}$. Neverthess, the assertion that $\operatorname{Eis}_{T}^{G} \simeq$ $\operatorname{Eis}_{M}^{G} \circ \operatorname{Eis}_{T}^{M}$ does hold. This is a non-trivial theorem (Theorem 2.3.10), which is one of the main results of this paper.

0.2.4. Finally, we have to describe the following two results, which are geometric interpretations of certain properties of the classical (i.e. function-theoretic) Eisenstein series operator.

\section{Behaviour with respect to the Hecke functors.}

Classically, on the space of functions on the double quotient $G_{\mathbb{O}} \backslash G_{\mathbb{A}} / G_{\mathcal{K}}$ we have the action of $\otimes \mathcal{H}_{x}(G)$, where $x$ runs over the set of places of $\mathcal{K}$, and for each $x \in X$, $\mathcal{H}_{x}(G)$ denotes the corresponding spherical Hecke algebra of the group $G$.

Similarly, $\underset{x \in X}{\otimes} \mathcal{H}_{x}(T)$ acts on the space of functions on $T_{\mathbb{O}} \backslash T_{\mathbb{A}} / T_{\mathcal{K}}$. In addition, for every $x$ as above, there is a canonical homomorphism $\mathcal{H}_{x}(G) \rightarrow \mathcal{H}_{x}(T)$ described as follows: 
Recall that there is a canonical isomorphism (due to Satake) between $\mathcal{H}_{x}(G)$ and the Grothendieck ring of the category of finite-dimensional representations of the Langlands dual group $\check{G}$. We have the natural restriction functor $\operatorname{Rep}(\check{G}) \rightarrow \operatorname{Rep}(\check{T})$, and our homomorphism $\mathcal{H}_{x}(G) \rightarrow \mathcal{H}_{x}(T)$ corresponds to the induced homomorphism $K(\operatorname{Rep}(\check{G})) \rightarrow K(\operatorname{Rep}(\check{T}))$ between Grothendieck rings.

The basic property of the Eisenstein series operators is that it intertwines the $\mathcal{H}_{x}(G)$ action on $G_{\mathbb{O}} \backslash G_{\mathbb{A}} / G_{\mathcal{K}}$ and the $\mathcal{H}_{x}(T)$-action on $T_{\mathbb{O}} \backslash T_{\mathbb{A}} / T_{\mathcal{K}}$ via the above homomorphism.

Our Theorem 2.1.5 is a reflection of this phenomenon in the geometric setting. Now, instead of the Hecke algebras, we have the action of the Hecke functors on $\operatorname{Sh}\left(\operatorname{Bun}_{G}\right)$. Namely, for $x \in X$ and an object $V \in \operatorname{Rep}(\check{G})$, one defines the Hecke functor

$$
\mathcal{S} \mapsto{ }_{x} H_{G}(V, \mathcal{S})
$$

from $\operatorname{Sh}\left(\operatorname{Bun}_{G}\right)$ to itself (cf. Sect. 3.2) and we have for $\mathcal{S} \in \operatorname{Sh}\left(\operatorname{Bun}_{T}\right)$ :

$$
{ }_{x} H_{G}(V, \operatorname{Eis}(\mathcal{S})) \simeq \operatorname{Eis}\left({ }_{x} H_{T}\left(\operatorname{Res}_{T}^{G}(V), \mathcal{S}\right)\right) .
$$

A similar statement holds for the non-principal Eisenstein series functor $\operatorname{Eis}_{M}^{G}$, cf. Theorem 2.3.7.

As a coroallary, we obtain that if $E_{\check{M}}$ is an $\check{M}$-local system on $X$ and $\operatorname{Aut}_{E_{\check{M}}}$ is a perverse sheaf (or a complex of sheaves) on $\operatorname{Bun}_{M}$, corresponding to it in the sense of the geometric Langlands correspondence, then the complex $\operatorname{Eis}_{M}^{G}\left(\operatorname{Aut}_{E_{\check{M}}}\right)$ on $\operatorname{Bun}_{G}$ is a Hecke eigen-sheaf with respect to the induced $\check{G}$-local system.

In particular, Theorem 2.1.5 establishes the geometric Langlands correspondence for those homomorphisms $\pi_{1}(X) \rightarrow \check{G}$, whose image is contained in a maximal torus of $\check{G}$.

\section{The functional equation.}

It is well-known that the classical Eisenstein series satisfy the functional equation. Namely, let $\chi$ be a character of the group $T_{\mathbb{O}} \backslash T_{\mathbb{A}} / T_{\mathcal{K}}$ and let $\mathrm{w} \in W$ be an element of the Weyl group. We can translate $\chi$ by menas of $\mathrm{w}$ and obtain a new (grossen)-character $\chi^{\mathrm{w}}$.

The functional equation is the assertion that the Eisenstein series corresponding to $\chi$ and $\chi^{\mathrm{w}}$ are equal, up to a ratio of the corresponding L-functions.

Now let $\mathcal{S}$ be an arbitrary complex of sheaves on $\mathrm{Bun}_{T}$ and let $\mathrm{w} \cdot \mathcal{S}$ be its w-translate. One may wonder whether there is any relation between $\operatorname{Eis}(\mathcal{S})$ and $\operatorname{Eis}(\mathrm{w} \cdot \mathcal{S})$.

We single out a subcategory in $\mathrm{Sh}\left(\operatorname{Bun}_{T}\right)$, corresponding to sheaves which we call "regular", for which we answer the above question. We claim (cf. Theorem 2.1.8) that for a regular $\mathcal{S}$

$$
\operatorname{Eis}(\mathcal{S}) \simeq \operatorname{Eis}(\mathrm{w} \cdot \mathcal{S}) .
$$

(It is easy to see that one should not expect the functional equation to hold for non-regular sheaves.)

A remarkable feature of this assertion is that the L-factors that enter the classical functional equation have disappeared. An explanation of this fact is provided by Theorem 2.2.12, which says that the definition of Eis via the intersection cohomology sheaf on $\overline{\mathrm{Bun}}_{B}$ already incorporates the $L$-function. 
We remark that an assertion similar to the above functional equation should hold also for non-principal Eisenstein series. Unfortunately, this is beyond access for our methods.

By combining Theorem 2.1.5 and Theorem 2.1.8 we obtain a proof of a special case of the Langlands conjecture. Namely, we prove Theorem 2.2.8, which says that if we start with an unramified irreducible representation of $\pi_{1}(X)$ into $\check{G}$, such that $\pi_{1}(X)^{\text {geom }}$ maps to $\check{T} \subset \check{G}$, then there exists an unramified automorphic form on $G_{\mathbb{A}}$ which corresponds to this representation in the sense of Langlands.

This may be considered as an application of the machinery developped in this paper to the classical theory of automorphic forms.

\subsection{Contents.}

0.3.1. Now let us briefly discuss how this paper is organized.

In Section 1 we discuss the definitions of Drinfeld's compactifications.

In Section 2 we formulate all the main theorems concerning the Eisenstein series functor. In the rest of the paper we deduce these theorems from various geometric properties of the stacks $\overline{\mathrm{Bun}}_{B}, \widetilde{\mathrm{Bun}}_{P}$ and their close relatives.

0.3.2. In Sections 3 and 4 our goal is to prove that our Eisenstein series functor commutes with the Hecke functors (cf. above). In Section 3 we consider the principal case, i.e. $P=B$ and in Section 4 we make a generalization to the case of a general parabolic subgroup.

Our discussion in these two sections is based on the Lusztig-Drinfeld-GinzburgMirkovic-Vilonen theory of spherical perverse sheaves on the affine Grassmannian $\mathrm{Gr}_{G}$, which we review in Sect. 3.2.

0.3.3. In Section 5 we formulate and prove Theorem 5.1.5, which is one of our main technical tools. It says that the stack $\overline{\operatorname{Bun}}_{B}$ (or, more generally, $\widetilde{\operatorname{Bun}}_{P}$ ), is locally acyclic with respect to the natural projection $\overline{\mathfrak{q}}: \overline{\operatorname{Bun}}_{B} \rightarrow \operatorname{Bun}_{T}\left(\right.$ resp., $\widetilde{\mathfrak{q}}_{P}: \widetilde{\operatorname{Bun}}_{P} \rightarrow \operatorname{Bun}_{M}$ ). In other words, this means that from the point of view of singularities, all the fibers of the above projections look the same.

Theorem 5.1.5 has two main applications. On the one hand, it implies Theorem 2.1.2, which says that the functor Eis commutes with Verdier duality. On the other hand, it is a key ingredient in the proof of Theorem 2.3.10 about the composition of Esenstein (cf. above).

0.3.4. In Sections 6 and 7 our goal is to prove of Theorem 2.3.10 and the functional equation.

Section 6 is largely preparatory; in it we introduce various stratifications of the stacks $\overline{\operatorname{Bun}}_{B}$ and $\widetilde{\operatorname{Bun}}_{P}$.

As we have mentioned before, the main problem in the proof of Theorem 2.3.10 is that the natural map $\operatorname{Bun}_{B} \rightarrow \operatorname{Bun}_{P}$ does not extend to a map from $\overline{\operatorname{Bun}}_{B}$ to $\operatorname{Bun}_{P}$. To cure this, we introduce the stack $\widetilde{\operatorname{Bun}}_{B, P}$, which will map to both $\overline{\operatorname{Bun}}_{B}$ and $\widetilde{\operatorname{Bun}}_{P}$. 
In Section 7 we prove Theorem 7.1.6, which asserts that the map $\widetilde{\operatorname{Bun}}_{B, P} \rightarrow \widetilde{\operatorname{Bun}}_{P}$ is stratified-small in the sense of Goresky-MacPherson. This will allow us to finally prove Theorem 2.3.10.

The paper is concluded by Sect. 7.3, in which we complete the proof of the functional equation (Theorem 2.1.8). As in the classical theory, after having established Theorem 2.3.10, it is sufficient to treat the case of reductive groups of semi-simple rank 1 , in which case the functional equatiuon isomorphism amounts to a Fourier-transform type argument, combined with the Serre duality.

0.4. Conventions. We will work over the base field $\mathbb{F}_{q}$ and study Weil sheaves over $\mathbb{F}_{q^{-}}$ schemes and stacks. However, all the results of this paper carry over to the characteristic 0 world, in which case one must replace $\overline{\mathbb{Q}_{\ell}}$-adic sheaves by holonomic D-modules.

0.4.1. The group $G$. Throughout the paper, $G$ will be a connected reductive group over $\mathbb{F}_{q}$. Moreover, we will assume that its derived group $G^{\prime}=[G, G]$ is simply connected.

We will fix a Borel subgroup $B \subset G$ and let $T$ be the "abstract" Cartan, i.e., $T=B / U$, where $U$ is the unipotent radical of $B$. We will denote by $\Lambda$ the coweight lattice of $T$ and by $\check{\Lambda}$-its dual, i.e. the weight lattice; $\langle\cdot, \cdot\rangle$ is the canonical pairing between the two.

The semi-group of dominant coweights (resp., weights) will be denoted by $\Lambda_{G}^{+}$(resp., by $\check{\Lambda}_{G}^{+}$). The set of vertices of the Dynkin diagram of $G$ will be denoted by J; for each $\mathfrak{i} \in \mathcal{J}$ there corresponds a simple coroot $\alpha_{i}$ and a simple root $\check{\alpha}_{i}$. The set of positive coroots will be denoted by $\Delta$ and their positive span inside $\Lambda$, by $\Lambda_{G}^{\text {pos }}$. (Note that, since $G$ is not semi-simple, $\Lambda_{G}^{+}$is not necessarily contained in $\Lambda_{G}^{\text {pos }}$.) By $\check{\rho} \in \check{\Lambda}$ we will denote the half sum of positive roots of $G$ and by w $\mathrm{w}_{0}$ the longest element in the Weyl group of $G$. For $\lambda_{1}, \lambda_{2} \in \Lambda$ we will write that $\lambda_{1} \geq \lambda_{2}$ if $\lambda_{1}-\lambda_{2} \in \Lambda_{G}^{\text {pos }}$, and similarly for $\check{\Lambda}_{G}^{+}$.

It will be convenient to choose fundamental weights $\check{\omega}_{\mathfrak{i}} \in \check{\Lambda}_{G}^{+}, \mathfrak{i} \in \mathcal{J}$ : each $\check{\omega}_{\mathfrak{i}}$ is non-uniquely characterized by the property that $\left\langle\alpha_{\mathfrak{j}}, \breve{\omega}_{\mathfrak{i}}\right\rangle=\delta_{\mathfrak{i}, \mathrm{j}}$.

Let $P$ be a standard parabolic of $G$, i.e. $P \supset B$; let $U(P)$ be its unipotent radical and $M:=P / U(P)$-the Levi factor (note that the derived group of $M$ is also simply connected). To $M$ there corresponds a subdiagram $\mathcal{J}_{M}$ in $\mathcal{J}$. We will denote by $\Lambda_{M}^{+} \supset$ $\check{\Lambda}_{G}^{+}, \Lambda_{M}^{\text {pos }} \subset \Lambda_{G}^{\text {pos }}, \check{\rho}_{M} \in \check{\Lambda}, \mathrm{w}_{0}^{M} \in W, \geq{ }_{M}$ etc. the corresponding objects for $M$.

To a dominant coweight $\check{\lambda} \in \check{\Lambda}$ one attaches the Weyl $G$-module, denoted $\mathcal{V}^{\check{\lambda}}$, with a fixed highest weight vector $v^{\check{\lambda}} \in \mathcal{V}^{\check{\lambda}}$. For a pair of weights $\check{\lambda}_{1}, \check{\lambda}_{2}$, there is a canonical map $\mathcal{V}^{\check{\lambda}_{1}+\check{\lambda}_{2}} \rightarrow \mathcal{V}^{\check{\lambda}_{1}} \otimes V^{\check{\lambda}_{2}}$ that sends $v^{\check{\lambda}_{1}+\check{\lambda}_{2}}$ to $v^{\check{\lambda}_{1}} \otimes v^{\check{\lambda}_{2}}$.

Similarly, for $\check{\nu} \in \check{\Lambda}_{M}^{+}$, we will denote by $\mathcal{U}^{\check{\nu}}$ the corresponding $M$-module. There is a natural functor $\mathcal{V} \mapsto \mathcal{V}^{U(P)}$ from the category of $G$-modules to that of $M$-modules and we have a canonical morphism $\mathcal{U}^{\check{\lambda}} \rightarrow\left(\mathcal{V}^{\check{\lambda}}\right)^{U(P)}$, which sends the corresponding highest weight vectors to one another.

By $\mathcal{F}_{G}$ we will denote principal $G$-bundles on various schemes. The notation $\mathcal{F}_{G}^{0}$ is reserved for the trivial $G$-bundle. For a $G$-bundle $\mathcal{F}_{G}$ (resp., a $T$-bundle $\mathcal{F}_{T}$ ) and a $G$ module $\mathcal{V}$ (resp., a character $\check{\lambda}: T \rightarrow \mathbb{G}_{m}$ ) let $\mathcal{V}_{\mathcal{F}_{G}}$ (resp., $\mathcal{L}_{\mathcal{F}_{T}}^{\check{\lambda}}$ ) denote the corresponding associated vector bundle (resp., line bundle). 
If $\mathcal{F}_{T}$ is a $T$-bundle on a scheme $y, \mu$ is a coweight of $T$ and $D \subset y$ is a Cartier divisor, we will denote by $\mathcal{F}_{T}(\mu \cdot D)$ the new $T$-bundle, defined by the condition that for a weight $\check{\mu}$ of $T$, the associated line bundle $\mathcal{L}_{\mathcal{F}_{T}(\mu \cdot D)}^{\check{\mu}}$ equals $\mathcal{L}_{\mathcal{F}_{T}}^{\check{\mu}}(\langle\mu, \check{\mu}\rangle \cdot D)$.

Finally, we let $\breve{G}$ denote the Langlands dual group of $G$. This is a reductive group over $\overline{\mathbb{Q}_{\ell}}$, endowed with a canonical Cartan subgroup $\check{T} \subset \check{G}$, whose lattice of characters is identified with $\Lambda$. For $\lambda \in \Lambda_{G}^{+}$, we will denote by $V^{\lambda}$ the corresponding $\check{G}$-module and for $\mu \in \Lambda, V^{\lambda}(\mu)$ will designate the $\mu$-th weight subspace of $V^{\lambda}$. For an $M$-dominant coweight $\nu$, we will denote the corresponding $\check{M}$-module by $U^{\nu}$.

0.4.2. Stacks. The main objects of study in this paper are various stacks attached to our curve $X$ (assumed to be smooth and complete) and the group $G$.

For instance, the stack of $G$-bundles on $X$, denoted $\operatorname{Bun}_{G}$, attaches to a scheme $S$ the groupoid of principal $G$-bundles on $S \times X$ and for a map of schemes $S_{1} \rightarrow S_{2}$ the corresponding functor is given by the ordinary pull-back.

In the main body of the paper, when giving a definition of a stack $y$, we will usually say that $y$ classifies something, by which we mean that it is clear what an $S$-family of this something is for a scheme $S$ and what the corresponding pull-back functor on the category of such families is, for a map $S_{1} \rightarrow S_{2}$. In addition, in all the examples that the reader will encounter in this paper, the fact that our $y$ is indeed an algebraic stack, follows easily from the fact that $\operatorname{Bun}_{G}$ is.

Here is an example:

The stack $y$ classifying the data of $(\mathcal{M}, s)$, where $\mathcal{M}$ is a coherent sheaf on $X$ and $s$ is its section (resp., an injective section) means that $\operatorname{Hom}(S, y)$ is the groupoid of pairs $\left(\mathcal{M}_{S}, s_{S}\right)$, where $\mathcal{M}_{S}$ is a coherent sheaf on $S \times X$, flat over $S$, and $s_{S}$ is a map $\mathcal{O}_{S \times X} \rightarrow \mathcal{M}_{S}$ (resp., a map, which is injective over any geometric point of $S$ ).

A special care must be used when we define stacks or schemes using the formal (resp., formal punctured) disc. We refer the reader to the Introduction of 13] for the corresponding conventions.

0.4.3. Derived categories. If $y$ is a scheme, we will denote by $\operatorname{Sh}(y)$ the corresponding bounded derived category of constructible $\overline{\mathbb{Q}_{\ell}}$-adic Weil sheaves on $y$ (cf. [9]). By abuse of language, we will call its objects "sheaves", but we will always mean complexes of such. The constant sheaf on $y$ will be denoted by $\overline{\mathbb{Q}_{\ell} y}$. We will denote by $\overline{\mathbb{Q}_{\ell}}\left(\frac{1}{2}\right)$ the 1-dimensional sheaf over $\operatorname{Spec}\left(\mathbb{F}_{q}\right)$ which corresponds to a chosen once and for all square root of $q$.

For a scheme or a stack $y$, Perv $(y)$ will denote the abelian category of perverse sheaves on $y$. ICy $\in \operatorname{Perv}(y)$ will denote the intersection cohomology sheaf on $y$, normalized so that it is pure of weight 0 . E.g., when $y$ is smooth, $\operatorname{IC}_{y}=\left(\overline{\mathbb{Q}_{\ell}}\left(\frac{1}{2}\right)[1]\right)_{y}^{\otimes} \operatorname{dim}(y)$. Unless specified otherwise, we work with the perverse t-structure, i.e. all the cohomological degrees are counted in the perverse normalization.

When $y$ is a stack, the definition of the derived category is problematic. However, this poses no difficulty in our case, since all our stacks are unions of open substacks of finite type, each being a quotient of a scheme by an affine group. If $y=\cup_{i} y_{i}$ is such 
a stack, then $\operatorname{Sh}(y):=\lim _{\leftarrow} \operatorname{Sh}\left(y_{i}\right)$, where each $\operatorname{Sh}\left(y_{i}\right)$ can be defined as an equivariant derived category in the sense of Bernstein-Lunts [4].

If $f: y_{1} \rightarrow y_{2}$ is a map of schemes (resp., of stacks) we will denote by $f_{!}, f_{*}, f^{*}, f^{!}$the corresponding functors between $\operatorname{Sh}\left(y_{1}\right)$ and $\operatorname{Sh}\left(y_{2}\right)$ always understood in the derived sense. Note, that if $f$ is non-representable, the functors $f_{!}$and $f_{*}$ do not in general preserve our bounded derived category. Luckily, in the main body of the paper, we will have to consider the above direct image functors only for representable morphisms (an exception being the definition of regular sheaves and the discussion in Sect. 7.3, in which case their usage will be but very mild).

Another warning on our abuse of language: let $f, f^{\prime}: y_{1} \rightarrow y_{2}$ be two morphisms of stacks. We will sometimes say that they coincide, by which we mean, of course, that they are isomorphic. At any event, in this situation, the pull-back functors $f^{*}$ and $f^{\prime *}$ from $\operatorname{Sh}\left(y_{2}\right) \rightarrow \operatorname{Sh}\left(y_{1}\right)$ are isomorphic too.

If $i: Z \hookrightarrow y$ is a locally closed embedding and $\mathcal{S} \in \operatorname{Sh}(y)$, the notation $\left.\mathcal{S}\right|_{Z}$ will mean the same as $i^{*}(\mathcal{S})$. The Verdier duality functor will be denoted by $\mathbb{D}$. For $\mathcal{S}$ as above, $h^{i}(\mathcal{S}) \in \operatorname{Perv}(\mathcal{y})$ is the $i$-th perverse cohomology of $\mathcal{S}$.

Let $\mathcal{F}_{H} \rightarrow \mathrm{y}$ be an $H$-torsor, where $H$ is an algebraic group and let $Z$ be an $H$ scheme. We will denote by $Z \stackrel{H}{\times} y$ the corresponding fibration over $y$ with the typical fiber $Z$. (The word "fibration" in this paper will be understood only in this sense.) Let $\mathcal{S}$ (resp., $\mathcal{T}$ ) be an object of $\operatorname{Sh}(Z)$ (resp., $\operatorname{Sh}(y)$ ) and assume, moreover, that $\mathcal{S}$ is $H$-equivariant. In this case we can form their twisted external product, denoted $\mathcal{S} \widetilde{\otimes} \mathcal{T}$, which will be an object of $\operatorname{Sh}(\underset{H}{*} \times$ y).

\section{Acknowledgements.}

This paper owes its existence to V. Drinfeld, who has explained to (one of) us the definition of $\overline{\mathrm{Bun}}_{B}$ and recommended to study it. Discussions with him and also with A. Beilinson and J. Bernstein helped us overcome many technical and ideological difficulties on the way.

Besides the work of Laumon, the present paper has been much influenced by the Beilinson-Drinfled work on the geometric Langlands correspondense and by the papers of Finkelberg and Mirkovic on semi-infinite flags.

\section{Definition of Drinfeld's COMPaCtifications}

\subsection{Strongly quasi-affine varieties.}

1.1.1. Let $Y$ be a scheme over an algebraically closed field $k$. We shall say that $Y$ is strongly quasi-affine if the following conditions hold:

1) The algebra $A_{Y}=\Gamma\left(Y, \mathcal{O}_{Y}\right)$ of global functions on $Y$ is finitely generated as a $k$-algebra.

2) Let $\bar{Y}=\operatorname{Spec}\left(A_{Y}\right)$. Then the natural map $Y \rightarrow \bar{Y}$ is an open embedding.

If $Y$ is strongly quasi-affine then we will call $\bar{Y}=\operatorname{Spec}(\mathcal{A})$ the affine closure of $Y$. In this section we will need the following result. 
Theorem 1.1.2. Let $G$ be a reductive group over $k$ and let $P$ be a parabolic subgroup of $G$. Let $H$ be either the derived group $[P, P]$ of $P$ or the unipotent radical $U(P)$ of $P$. Let $Y=G / H$. Then

1. $Y$ is a strongly quasi-affine variety.

2. For any scheme $S$ over $k$ an $S$-point of $\bar{Y}$ is described by a collection of maps of $\mathcal{O}_{S}$-modules $\kappa^{\mathcal{V}}: \mathcal{O}_{S} \otimes \mathcal{V}^{H} \rightarrow \mathcal{O}_{S} \otimes \mathcal{V}$ for every $G$-module $\mathcal{V}$ satisfying the following conditions:

a) If $\mathcal{V}$ is the trivial representation of $G$ then $\kappa^{\mathcal{V}}=i d$.

b) Assume that we are given a morphism $a: V^{1} \rightarrow V^{2}$ of $G$-modules. Then the following square must be commutative:

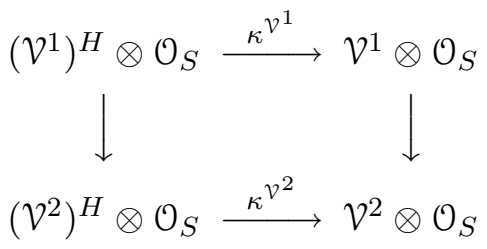

c) For a pair of $G$-modules, $\mathcal{V}^{1}$ and $\mathcal{V}^{2}$, the square

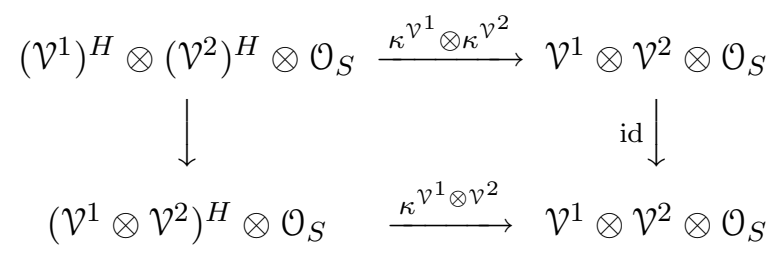

should commute too.

The second assertion of Theorem 1.1 .2 is straightforward for any $H$ for which $G / H$ is strongly quasi-affine. The first assertion is straighforward when $H=[P, P]$ and when $H=U(P)$ this is a theorem of F. D. Grosshans (cf. [17]).

1.1.3. Let $G, H$ and $Y$ be as above. It will be convenient to describe $S$-points of $Y$ slightly differently. For a $P / H$-module $\mathcal{U}$, let $\operatorname{Ind}(\mathcal{U})$ denote the $G$-module induced from the corresponding $P$-module, i.e. for a $G$-module $\mathcal{V}$, we have functorially:

$$
\operatorname{Hom}_{P / H}\left(\mathcal{U}, \mathcal{V}^{H}\right) \simeq \operatorname{Hom}_{G}(\operatorname{Ind}(\mathcal{U}), \mathcal{V}) .
$$

From Theorem 1.1.2 we obtain using the Frobenius reciprocity:

Lemma 1.1.4. For a $k$-scheme $S$, an $S$-point of $\bar{Y}$ is the same as a collection of maps of $\mathcal{O}_{S}$-modules $\kappa^{\mathfrak{U}}: \mathcal{O}_{S} \otimes \mathfrak{U} \rightarrow \mathcal{O}_{S} \otimes \operatorname{Ind}(\mathcal{U})$ for every $P / H$-module $\mathfrak{U}$ satisfying the following conditions:

a) If $\mathcal{U}$ is the trivial representation of $P / H$ then $\operatorname{Ind}(\mathcal{U})$ is canonically trivial as well and $\kappa^{\mathrm{U}}$ must equal the identity map.

b) Assume that we are given a morphism a: $\mathcal{U}^{1} \rightarrow \mathcal{U}^{2}$ of $G$-modules. Then the following square must be commutative: 


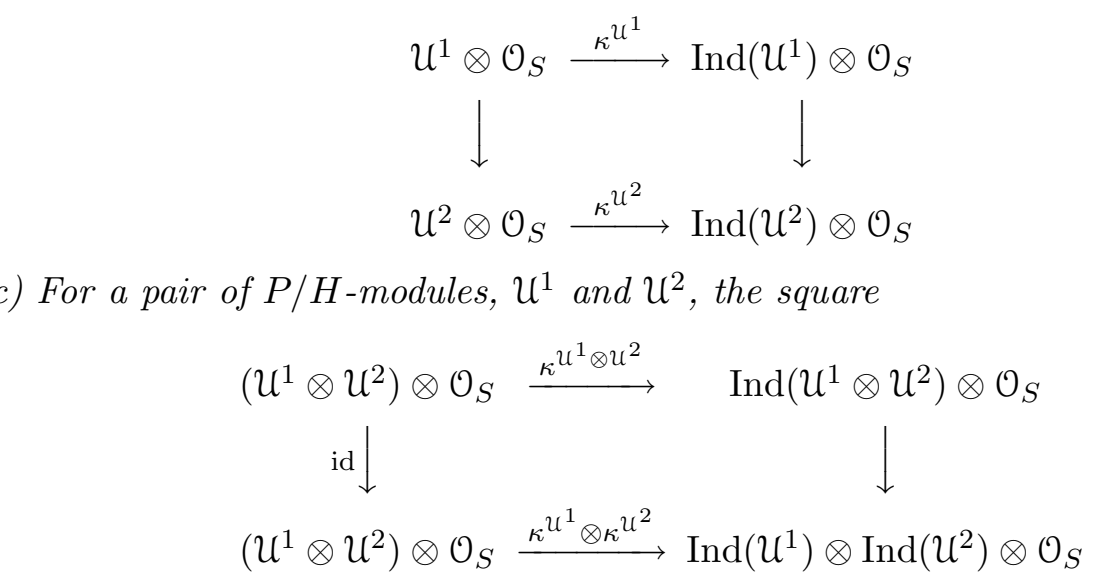

should commute too.

1.2. Drinfeld structures. Consider the stacks $\operatorname{Bun}_{G}, \mathrm{Bun}_{B}$ and $\mathrm{Bun}_{T}$. The maps of groups $B \hookrightarrow G$ and $B \rightarrow T$ give rise to a diagram of stacks:

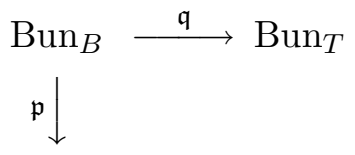

$\operatorname{Bun}_{G}$.

All the three stacks are smooth. It is easy to see, that the projection $\mathfrak{q}$ is smooth, but in general non-representable and that the projection $\mathfrak{p}$ is representable, but neither smooth nor proper.

Our first goal is to define (following Drinfeld) a compactification of $\operatorname{Bun}_{B}$ along the fibers of the projection $\mathfrak{p}$; in other words we will construct a stack $\overline{\operatorname{Bun}}_{B}$ which contains $\operatorname{Bun}_{B}$ as an open sub-stack and which is endowed with projections

$$
\overline{\mathfrak{p}}: \overline{\operatorname{Bun}}_{B} \rightarrow \operatorname{Bun}_{G} \text { and } \overline{\mathfrak{q}}: \overline{\operatorname{Bun}}_{B} \rightarrow \operatorname{Bun}_{T},
$$

such that the map $\overline{\mathfrak{p}}$ is representable is proper.

1.2.1. Note that the stack Bun $_{B}$, by definition, classifies the following data:

$$
\left(\mathcal{F}_{G} ; \mathcal{F}_{T} ; \kappa: \mathcal{F}_{G} \rightarrow G / U^{T} \times \mathcal{F}_{T}\right),
$$

where $\mathcal{F}_{G}$ is a $G$-bundle, $\mathcal{F}_{T}$ is a $T$-bundle and $\kappa$ is a $G$-equiavariant map.

Recall that $G / U$ is a strongly quasi-affine variety and let $\overline{G / U}$ denote its affine closure. The groups $G$ and $T$ act on $G / U$ and therefore also on $\overline{G / U}$.

We define the functor $\overline{\operatorname{Bun}}_{B}$ on the category of $k$-schemes as follows: an $S$-point of $\overline{\operatorname{Bun}}_{B}$ is a triple $\left(\mathcal{F}_{G}, \mathcal{F}_{T}, \kappa\right)$, where $\mathcal{F}_{G}$ (resp., $\mathcal{F}_{T}$ ) is an $S$-point of $\operatorname{Bun}_{G}$ (resp., of $\operatorname{Bun}_{T}$ ) and $\kappa$ is a $G$-equivariant map

$$
\mathcal{F}_{G} \rightarrow \overline{G / U}^{T} \times \mathcal{F}_{T},
$$


such that for every geometric point $s \in S$ there is a Zariski-open subset $X^{0} \subset X \times s$ such that the map

$$
\left.\kappa\right|_{X^{0}}:\left.\left.\mathcal{F}_{G}\right|_{X^{0}} \rightarrow \overline{G / U}^{T} \mathcal{F}_{T}\right|_{X^{0}}
$$

factors through $G / U^{T} \times\left.\mathcal{F}_{T}\right|_{X^{0}} \subset \overline{G / U}^{T} \times\left.\mathcal{F}_{T}\right|_{X^{0}}$.

In the sequel, we will simply say that $\overline{\mathrm{Bun}}_{B}$ classifies the data of $\left(\mathcal{F}_{G} ; \mathcal{F}_{T} ; \kappa: \mathcal{F}_{G} \rightarrow\right.$ $\left.\overline{G / U}^{T} \times \mathcal{F}_{T}\right)$.

In order to show that $\overline{\mathrm{Bun}}_{B}$ is an algebraic stack in the smooth topology with the desired properties, we will slightly rewrite the above definition. Let us apply Lemma 1.1.4 in our case when $H=U$. Since $P / H=B / U=T$ is commutative, it is enough to consider only 1-dimensional $P / H$-modules $\mathcal{U}$. If $\check{\lambda}$ is a character of $T$, the corresponding induced $G$-module vanishes unless $\check{\lambda} \in \check{\Lambda}_{G}^{+}$and in the latter case it equals $\mathcal{V}^{\check{\lambda}}$.

From Lemma 1.1.4 we obtain that an $S$-point of $\overline{\mathrm{Bun}}_{B}$ is a triple $\left(\mathcal{F}_{G}, \mathcal{F}_{T}, \kappa^{\check{\lambda}}, \forall \check{\lambda} \in\right.$ $\check{\Lambda}_{G}^{+}$), where $\mathcal{F}_{G}$ and $\mathcal{F}_{T}$ are as above, and $\kappa^{\check{\lambda}}$ is a map of coherent sheaves

$$
\mathcal{L}_{\mathcal{F}_{T}}^{\check{L}} \hookrightarrow \mathcal{V}_{\mathcal{F}_{G}}^{\check{\lambda}},
$$

(cf. our conventions in Sect. 0.4) such that for evey geometric point $s \in S$ the restriction $\left.\kappa^{\check{\lambda}}\right|_{X \times s}$ is an injection. The last condition is equivalent to saying that $\kappa^{\check{\lambda}}$ is an injection such that the quotient $\mathcal{V}_{\mathfrak{F}_{G}}^{\check{L}} / \operatorname{Im}\left(\kappa^{\check{\lambda}}\right)$ is $S$-flat.

The system of embeddings $\kappa^{\check{\lambda}}$ must satisfy conditions (a)-(c) of Lemma 1.1.4. Let us write them down explicitly in our case. We obtain the so-called Plücker relations:

First, for $\check{\lambda}=0, \kappa^{0}$ must be the identity map $\mathcal{O} \simeq \mathcal{L}_{\mathcal{F}_{T}}^{0} \rightarrow \mathcal{V}_{\mathcal{F}_{G}}^{0} \simeq \mathcal{O}$. Secondly, for two dominant integral weights $\check{\lambda}$ and $\check{\mu}$, the map

$$
\mathcal{L}_{\mathcal{F}_{T}}^{\check{\lambda}} \otimes \mathcal{L}_{\mathcal{F}_{T}}^{\check{\mu}} \stackrel{\kappa^{\check{\lambda}} \otimes \kappa^{\check{\mu}}}{\longrightarrow} \mathcal{V}_{\mathcal{F}_{G}}^{\check{\lambda}} \otimes \mathcal{V}_{\mathcal{F}_{G}}^{\check{\mu}} \simeq\left(\mathcal{V}^{\check{\lambda}} \otimes \mathcal{V}^{\check{\mu}}\right)_{\mathcal{F}_{G}}
$$

must coincide with the composition

$$
\mathcal{L}_{\mathcal{F}_{T}}^{\check{\lambda}} \otimes \mathcal{L}_{\mathcal{F}_{T}}^{\check{\mu}} \simeq \mathcal{L}_{\mathcal{F}_{T}}^{\check{\lambda}+\check{\mu}} \stackrel{\check{\lambda}+\check{\mu}}{\longrightarrow} \mathcal{V}_{\mathcal{F}_{G}}^{\check{\lambda}+\check{\mu}} \rightarrow\left(\mathcal{V}^{\check{\lambda}} \otimes \mathcal{V}^{\check{\mu}}\right)_{\mathcal{F}_{G}}
$$

Proposition 1.2.2. The functor $\overline{\mathrm{Bun}}_{B}$ is an algebraic stack in the smooth topology. The natural map $\overline{\operatorname{Bun}}_{B} \rightarrow \operatorname{Bun}_{G}$ given by $\left(\mathcal{F}_{G}, \mathcal{F}_{T}, \kappa\right) \mapsto \mathcal{F}_{G}$ is representable and proper.

Proof. Let $\mathcal{F}_{G}$ be an $S$-point of Bun $_{G}$. We have to prove that the Cartesian product $S \underset{\operatorname{Bun}_{G}}{\times} \overline{\operatorname{Bun}}_{B}$ is representable by a scheme proper over $S$.

For a weight $\check{\lambda}$ consider the Hilbert scheme Hilb ${ }^{\check{\lambda}}$ whose $S^{\prime}$ points are pairs:

(A map $f: S^{\prime} \rightarrow S$; a subsheaf $\mathcal{L}$ of $f^{*}\left(\mathcal{V}_{\mathcal{F}_{G}}^{\lambda}\right)$ on $X \times S^{\prime}$ such that $\mathcal{L}$ is locally free of rank 1 and the quotient $f^{*}\left(\mathcal{V}_{\mathcal{F}_{G}}^{\lambda}\right) / \mathcal{L}$ is $S^{\prime}$-flat.)

It is well-known that Hilb ${ }^{\check{\lambda}}$ exists and is proper over $S$. Now, let $\left(\mathcal{F}_{G}, \mathcal{F}_{T}, \kappa\right)$ be an $S^{\prime}$-point of $S \underset{\operatorname{Bun}_{G}}{\times} \overline{\operatorname{Bun}}_{B}$. To it we can attach an $S^{\prime}$-point of Hilb ${ }^{\check{\lambda}}$ for every $\check{\lambda}$, by setting $\mathcal{L}=\mathcal{L}_{\mathcal{F}_{T}}^{\check{\lambda}}$. 
Moreover, since every collection of $\left\{\kappa^{\check{\lambda}}\right\}$ 's satisfying the Plücker relations is uniquely determined by its values on the fundamental weights, i.e. by the $\kappa^{\check{\omega}_{\mathfrak{i}}}$ 's, $\mathfrak{i} \in \mathcal{J}$, we obtain that the above morphisms of functors define a closed embedding

$$
S \underset{\operatorname{Bun}_{G}}{\times} \overline{\operatorname{Bun}}_{B} \hookrightarrow \underset{\mathfrak{i} \in \mathcal{J}}{\times \operatorname{Hillb}^{\tilde{\omega}_{\mathfrak{i}}}} .
$$

Therefore, our Cartesian product is also representable by a scheme proper over $S$.

Obviously, we have an open embedding of $\operatorname{Bun}_{B}$ into $\overline{\mathrm{Bun}}_{B}$. The image corresponds

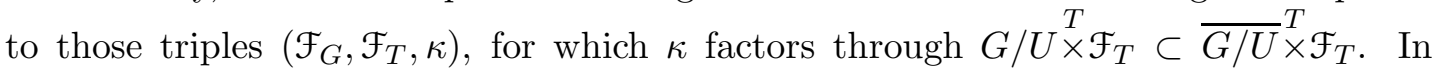
terms of the embeddings $\kappa^{\check{\lambda}}$, an $S$-point belongs to $\operatorname{Bun}_{B}$ if and only if each

$$
\kappa^{\check{\lambda}}: \mathcal{L}_{\mathcal{F}_{T}}^{\check{\lambda}} \rightarrow \mathcal{V}_{\mathcal{F}_{G}}^{\check{\lambda}}
$$

is a bundle map, i.e. the quotient is not only $S$-flat, but is in fact $X \times S$-flat. We will denote this open embedding by $j_{B}$, or simply by $j$.

We will denote the natural map $\overline{\operatorname{Bun}}_{B} \rightarrow \operatorname{Bun}_{G}$ by $\overline{\mathfrak{p}}$, since it extends the map $\mathfrak{p}: \operatorname{Bun}_{B} \rightarrow \operatorname{Bun}_{G}$. The map $\left(\mathcal{F}_{G}, \mathcal{F}_{T}, \kappa\right) \mapsto \mathcal{F}_{T}: \overline{\operatorname{Bun}}_{B} \rightarrow \operatorname{Bun}_{T}$ will be denoted by $\overline{\mathfrak{q}}$ for the same reason.

Proposition 1.2.3. $\operatorname{Bun}_{B}$ is dense in $\overline{\mathrm{Bun}}_{B}$.

The proof will be given in Sect. 6.1. We remark that the simply-connectedness assumption on $G^{\prime}$ was necessary precisely in order to make Proposition 1.2.3 true.

1.2.4. We will call $\overline{\mathbb{F}_{q}}$-valued points of $\overline{\operatorname{Bun}}_{B}$ "Drinfeld's structures". Let us see explicitly what they look like.

Note on the terminology: If $\mathcal{M}_{1}$ and $\mathcal{M}_{2}$ are vector bundles on a scheme $y, \kappa$ : $\mathcal{M}_{1} \rightarrow \mathcal{M}_{2}$ is an embedding of coherent sheaves and $Z \subset y$ is a closed subscheme, we will say that $\kappa$ has no zeroes on $Z$ or is maximal at $Z$ if the following equiavlent conditions hold:

1. The quotient $\mathcal{M}_{2} / \mathcal{M}_{1}$ has no torsion supported on $Z$.

2. There is no non-trivial $\mathcal{N}$ with $\mathcal{M}_{1} \subset \mathcal{N} \subset \mathcal{M}_{2}$, such that $\mathcal{N} / \mathcal{M}_{1}$ is a torsion sheaf supported on $Z$.

Proposition 1.2.5. Let $\left(\mathcal{F}_{G}, \mathcal{F}_{T}, \kappa\right)$ be an $\overline{\mathbb{F}_{q}}$-point of $\overline{\operatorname{Bun}}_{B}$. Then

(1) There is a Zariski-open subset $X^{0} \subset X$, such $\left(\mathcal{F}_{G}, \mathcal{F}_{T}, \kappa\right)$ defines a reduction of $\left.\mathcal{F}_{G}\right|_{X^{0}}$ to $B$.

(2) There exists a canonical principal B-bundle $\mathcal{F}_{B}^{\prime}$ on $X$, such that $\left.\mathcal{F}_{B}^{\prime}\right|_{X^{0}}$ is the $B$ bundle of point (1).

Proof. Consider the embeddings $\kappa^{\check{\omega}_{\mathfrak{i}}}: \mathcal{L}_{\mathcal{F}_{T}}^{\check{\mathcal{T}}_{\mathrm{i}}} \rightarrow \mathcal{V}_{\mathcal{F}_{G}}^{\check{\omega}_{\mathrm{i}}}, \mathfrak{i} \in \mathcal{J}$. We take $X^{0}$ to be the maximal open subset of $X$, such that none of the above $\kappa^{\check{\omega}_{i}}$ 's has zeroes on $x \in X^{0}$.

From the Plücker relations, we obtain that on $X^{0}$, the other $\kappa^{\check{\lambda}}$ 's have no zeroes either. Hence, $\mathcal{F}_{B}:=\left(\left.\mathcal{F}_{G}\right|_{X^{0}},\left.\mathcal{F}_{T}\right|_{X^{0}},\left.\kappa\right|_{X^{0}}\right)$ is the thought-for $B$-bundle on $X^{0}$.

Let $X-X^{0}=\left\{x_{1}, \ldots, x_{n}\right\}$. To each of the points $x_{k}$, we can assign an element $\nu_{k} \in \Lambda_{G}^{\text {pos }}$. Namely, $\nu_{k}$ is such that $\left\langle\nu_{k}, \check{\omega}_{\mathfrak{i}}\right\rangle$ is the order of zero of $\kappa^{\check{\omega}_{\mathfrak{i}}}$ at $x_{k}$. 
If we put $\mathcal{F}_{T}^{\prime}=\mathcal{F}_{T}\left(\sum_{k} \nu_{k} \cdot x_{k}\right)$, we obtain a system of maximal embeddings

$$
\kappa^{\prime \check{\omega}_{i}}: \mathcal{L}_{\mathcal{F}_{T}^{\prime}}^{\check{\omega}_{i}} \rightarrow \mathcal{V}_{\mathcal{F}_{G}}^{\check{\omega}_{i}}
$$

satisfying the Plücker relations.

Hence $\mathcal{F}_{B}^{\prime}:=\left(\mathcal{F}_{G}, \mathcal{F}_{T}^{\prime}, \kappa^{\prime}\right)$ is a $B$-bundle on $X$, which on $X^{0}$ coincides with $\mathcal{F}_{B}$.

Let $\left(\mathcal{F}_{G}, \mathcal{F}_{T}, \kappa\right),\left\{x_{1}, \ldots, x_{n}\right\}$ and $\left\{\nu_{1}, \ldots, \nu_{k}\right\}$ be as above. We will say that our Drinfeld's structure has singularities on $X-X^{0}$ and we will call $\nu_{k}$ its defect at $x_{k}$. The $B$-bundle $\mathcal{F}_{B}$ will be called the saturation of $\left(\mathcal{F}_{G}, \mathcal{F}_{T}, \kappa\right)$.

1.3. Parabolic Drinfeld's structures. Let us now generalize the above definitions to the case, when $B \subset G$ is replaced by a parabolic subgroup $P$. In this case, there will be two different compactifications of the stack $\operatorname{Bun}_{P}$ along the fibers of the projection $\operatorname{Bun}_{P} \rightarrow \operatorname{Bun}_{G}$.

1.3.1. For a parabolic $P$, let $U(P), M$ and $\mathcal{J}_{M}$ be as in Sect. 0.4. We will denote by $\Lambda_{G, P}$ the quotient lattice

$$
\Lambda / \operatorname{Span}\left\{\alpha_{\mathfrak{i}}, \mathfrak{i} \in \mathcal{J}_{M}\right\} .
$$

Let $\check{\Lambda}_{G, P}$ denote the dual lattice. In other words, $\check{\Lambda}_{G, P}$ is the lattice of characters of the torus $M /[M, M]=P /[P, P]$. We have:

$$
\check{\Lambda}_{G, P}=\left\{\check{\lambda} \in \check{\Lambda} \mid\left\langle\alpha_{\mathfrak{i}}, \check{\lambda}\right\rangle=0 \text { if } \mathfrak{i} \in \mathcal{J}_{M}\right\} .
$$

Let $\Lambda_{G, P}^{\text {pos }}$ denote the positive part of $\Lambda_{G, P}$, i.e. the span of the projections of $\alpha_{\mathfrak{i}}$, $\mathfrak{i} \in \mathcal{J}-\mathcal{J}_{M}$.

1.3.2. The stack Bun $_{P}$ classifies the data of

$$
\left(\mathcal{F}_{G}, \mathcal{F}_{M /[M, M]}, \kappa_{P}: \mathcal{F}_{G} \rightarrow G /[P, P] \stackrel{M /[M, M]}{\times} \mathcal{F}_{M /[M, M]}\right),
$$

where $\mathcal{F}_{G}$ (resp., $\mathcal{F}_{M /[M, M]}$ ) is a $G$-bundle (resp., an $M /[M, M]$-bundle) and $\kappa$ is a $G$-equivariant map.

We let $\mathfrak{q}_{P}^{\dagger}$ (resp., $\mathfrak{p}_{P}$ ) denote the natural projection $\operatorname{Bun}_{P} \rightarrow \operatorname{Bun}_{M /[M, M]}$ (resp., $\left.\operatorname{Bun}_{P} \rightarrow \operatorname{Bun}_{G}\right)$. As in the case of the Borel subgroup, the projection $\mathfrak{q}_{P}^{\dagger}$ is smooth (but non-representable) and the projection $\mathfrak{p}_{P}$ is representable, but neither smooth nor proper.

The variety $G /[P, P]$ is strongly quasi-affine and let $\overline{G /[P, P]}$ denote its affine closure. We let $\overline{\operatorname{Bun}}_{P}$ be the stack that classifies triples

$$
\left(\mathcal{F}_{G}, \mathcal{F}_{M /[M, M]}, \kappa_{P}: \mathcal{F}_{G} \rightarrow \overline{G /[P, P]}^{M /[M, M]} \mathcal{F}_{M /[M, M]}\right),
$$

where $\mathcal{F}_{G}$ and $\mathcal{F}_{M /[M, M]}$ are as above and $\kappa_{P}$ is a $G$-equivariant map, which over the generic point of $X$ maps $\mathcal{F}_{G}$ to

$$
G /[P, P] \stackrel{M /[M, M]}{\times} \mathcal{F}_{M /[M, M]} \subset \overline{G /[P, P]}^{M /[M, M]} \stackrel{\times}{\times} \mathcal{F}_{M /[M, M]},
$$

in the same sense as in the definition of $\overline{\operatorname{Bun}}_{B}$.

Here is a Plücker-type description of $\overline{\mathrm{Bun}}_{P}$ : 
An $S$-point of $\overline{\operatorname{Bun}}_{P}$ consists of $\left(\mathcal{F}_{G}, \mathcal{F}_{M /[M, M]}, \kappa_{P}^{\check{\lambda}}, \forall \check{\lambda} \in \check{\Lambda}_{G, P} \cap \Lambda_{G}^{+}\right)$, where $\mathcal{F}_{G}$ and $\mathcal{F}_{M /[M, M]}$ are as above, and $\kappa_{P}^{\check{\lambda}}$ are maps of coherent sheaves

$$
\mathcal{L}_{\mathcal{F}_{M /[M, M]}^{\check{\nu}}}^{\check{L}} \hookrightarrow \mathcal{V}_{F_{G}}^{\check{\lambda}},
$$

which satisfy the same conditions as in the case of $\overline{\mathrm{Bun}}_{B}$.

Similarly to the previous case, we obtain that $\overline{\mathrm{Bun}}_{P}$ is indeed an algebraic stack, representable and proper over $\operatorname{Bun}_{G}$. We will denote by $\overline{\mathfrak{p}}_{P}$ the natural map $\overline{\operatorname{Bun}}_{P} \rightarrow \operatorname{Bun}_{G}$ and by $\overline{\mathfrak{q}}_{P}^{\dagger}$ the map $\overline{\operatorname{Bun}}_{P} \rightarrow \operatorname{Bun}_{M /[M, M]}$ given by $\left(\mathcal{F}_{G}, \mathcal{F}_{M /[M, M]}, \kappa_{P}\right) \mapsto \mathcal{F}_{M /[M, M]}$. By $j_{P}$ we will denote the open embedding $\operatorname{Bun}_{P} \hookrightarrow \overline{\operatorname{Bun}}_{P}$.

1.3.3. We will call $\overline{\mathbb{F}_{q}}$-points of $\overline{\operatorname{Bun}}_{P}$ "parabolic Drinfeld's structures". An ana$\log$ of Proposition 1.2.5 holds in the parabolic case as well. Namely, to an $\overline{\mathbb{F}_{q}}$-point $\left(\mathcal{F}_{G}, \mathcal{F}_{M /[M, M]}, \kappa_{P}\right)$ of $\overline{\mathrm{Bun}}_{P}$ one can attach a finite collection of points $x_{1}, \ldots, x_{n} \in X$ and elements $\nu_{1}, \ldots, \nu_{n} \in \Lambda_{G, P}^{\text {pos }}$, such that:

On $X^{0}:=X-\left\{x_{1}, \ldots x_{n}\right\},\left(\mathcal{F}_{G}, \mathcal{F}_{M /[M, M]}, \kappa_{P}\right)$ defines a $P$-bundle. This $P$-bundle can be extended to a $P$-bundle $\mathcal{F}_{P}^{\prime}=\left(\mathcal{F}_{G}, \mathcal{F}_{M /[M, M]}^{\prime}, \kappa_{P}^{\prime}\right)$ on the entire $X$ with $\mathcal{F}_{M /[M, M]}^{\prime}=$ $\mathcal{F}_{M /[M, M]}\left(\sum_{k} \theta_{k} \cdot x_{k}\right)$. We will call this $\mathcal{F}_{P}^{\prime}$ the saturation of $\left(\mathcal{F}_{G}, \mathcal{F}_{M /[M, M]}, \kappa_{P}\right)$.

1.3.4. Observe now that the inclusion of groups $B \subset P$ gives rise to a map of stacks $\operatorname{Bun}_{B} \rightarrow \operatorname{Bun}_{P}$. One may wonder whether this map extends to a map between $\overline{\operatorname{Bun}}_{B}$ and $\overline{\mathrm{Bun}}_{P}$. In this case the answer is positive:

Using the natural maps $T \rightarrow M /[M, M]$ and $\overline{G / U} \rightarrow \overline{G /[P, P]}$, to every $S$-point $\left(\mathcal{F}_{G}, \mathcal{F}_{T}, \kappa\right)$ of $\overline{\operatorname{Bun}}_{B}$ we can assign in a functorial way an $S$-point $\left(\mathcal{F}_{G}, \mathcal{F}_{M /[M, M]}, \kappa_{P}\right)$ of $\overline{\operatorname{Bun}}_{P}$. We will denote this map of stacks by $\mathfrak{s}_{P}$. In terms of the Plücker picture, $\mathfrak{s}_{P}$ "remembers" only the subsheaves $\mathcal{L}_{\mathcal{F}_{T}}^{\check{\lambda}} \hookrightarrow \mathcal{V}_{\mathcal{F}_{G}}^{\check{\lambda}}$ for $\check{\lambda} \in \check{\Lambda}_{G, P} \cap \check{\Lambda}_{G}^{+}$.

We have $\overline{\mathfrak{p}}=\overline{\mathfrak{p}}_{P} \circ \mathfrak{s}_{p}$. This implies, in particular, that $\mathfrak{s}_{P}$ is representable and proper.

In addition to the map $\mathfrak{q}_{P}^{\dagger}: \operatorname{Bun}_{P} \rightarrow \operatorname{Bun}_{M /[M, M]}$, there is a natural map $\mathfrak{q}_{P}$ : $\operatorname{Bun}_{P} \rightarrow \operatorname{Bun}_{M}$; one can show that this map does not extend to a map of stacks

$$
\overline{\operatorname{Bun}}_{P} \rightarrow \operatorname{Bun}_{M}
$$

We will construct now another relative compactification $\widetilde{\operatorname{Bun}}_{P}$ of $\operatorname{Bun}_{P}$, such that $\mathfrak{q}_{P}$ will extend to a map $\widetilde{\mathfrak{q}}_{P}: \widetilde{\operatorname{Bun}}_{P} \rightarrow \operatorname{Bun}_{M}$.

The stack $\widetilde{\operatorname{Bun}}_{P}$ will project naturally to $\overline{\operatorname{Bun}}_{P}$; however, the map $\mathfrak{s}_{P}: \overline{\operatorname{Bun}}_{B} \rightarrow$ $\overline{\operatorname{Bun}}_{P}$ will not lift to a map $\overline{\operatorname{Bun}}_{B} \rightarrow \widetilde{\operatorname{Bun}}_{P}$.

1.3.5. The stack Bun $_{P}$ can be also viewed as a stack that classifies triples

$$
\left(\mathcal{F}_{G}, \mathcal{F}_{M}, \widetilde{\kappa}_{P}: \mathcal{F}_{G} \rightarrow G / U(P) \stackrel{M}{\times} \mathcal{F}_{M}\right),
$$

where $\mathcal{F}_{G}$ and $\mathcal{F}_{M}$ are $G$ - and $M$-bundles respectively and where $\widetilde{\kappa}_{P}$ is a $G$-equivariant map. The variety $G / U(P)$ is strongly quasi-affine, according to Theorem 1.1.2, and let $\overline{G / U(P)}$ denote its affine closure. 
We set $\widetilde{\operatorname{Bun}}_{P}$ to be the stack that classifies triples

$$
\left(\mathcal{F}_{G}, \mathcal{F}_{M}, \widetilde{\kappa}_{P}: \mathcal{F}_{G} \rightarrow \overline{G / U(P)}^{M} \times \mathcal{F}_{M}\right),
$$

where $\widetilde{\kappa}_{P}$ is a $G$-equivariant map, such that over the generic point of $X, \mathcal{F}_{G}$ is mapped to

$$
G / U(P) \stackrel{M}{\times} \mathcal{F}_{M} \subset \overline{G / U(P)} \times \stackrel{M}{\times} \mathcal{F}_{M},
$$

as in the definition of $\overline{\operatorname{Bun}}_{B}$.

To prove that $\widetilde{\operatorname{Bun}}_{P}$ is indeed an algebraic stack, representable and proper over $\operatorname{Bun}_{G}$ we will once again have to resort to the Plücker picture:

By Theorem 1.1.2, as $S$-point of $\widetilde{\operatorname{Bun}}_{P}$ is a triple $\left(\mathcal{F}_{G}, \mathcal{F}_{M}, \widetilde{\kappa}_{P}^{\mathcal{V}}\right)$, where $\widetilde{\kappa}_{P}^{\mathcal{V}}$ is a map of coherent sheaves, defined for every $G$-module $\mathcal{V}$ :

$$
\left(\mathcal{V}^{U(P)}\right)_{\mathcal{F}_{M}} \rightarrow \mathcal{V}_{\mathcal{F}_{G}}
$$

such that

1) For every geometric point $s \in S$, the restriction $\left.\widetilde{\kappa}_{P}^{\mathcal{V}}\right|_{X \times s}$ is an injection.

2) Plücker relations hold in the following sense:

a) For the trivial representation $\mathcal{V}, \widetilde{\kappa}_{P}^{\mathcal{V}}$ must be the identity map $\mathcal{O} \rightarrow \mathcal{O}$.

b) For a $G$-module map $\nu^{1} \rightarrow \mathcal{V}^{2}$ the square

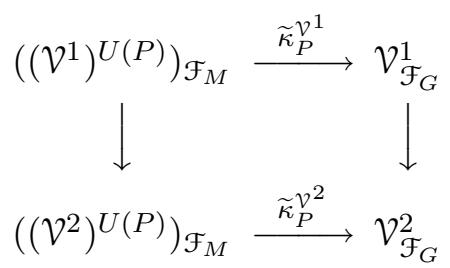

should commute.

c) For a pair of $G$-modules, $\mathcal{V}^{1}$ and $\mathcal{V}^{2}$, the square

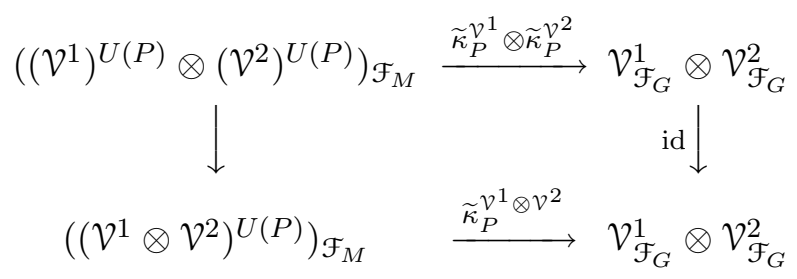

should commute too.

Proposition 1.3.6. The morphism of functors $\widetilde{\operatorname{Bun}}_{P} \rightarrow \operatorname{Bun}_{G}$ is representable and proper.

Proof. The proof will use the same idea as the proof of Proposition 1.2.2, of which the latter is a particular case, since for $P=B, \overline{\operatorname{Bun}}_{P}=\widetilde{\operatorname{Bun}}_{P}=\overline{\operatorname{Bun}}_{B}$.

Let $\mathcal{V}$ be a rerpesentation of $G$; put $n=\operatorname{dim}(\mathcal{V}), k=\operatorname{dim}\left(\mathcal{V}^{U(P)}\right)$. Let $\operatorname{Bun}_{n}=$ $\operatorname{Bun}_{G L(n)}$ denote the stack of rank $n$ vector bundles on $X$ and let $\operatorname{Bun}_{k, n}$ denote the stack classifying pairs $\mathcal{F}_{k} \hookrightarrow \mathcal{F}_{n}$ where $\mathcal{F}_{n}$ is a rank $n$ vector bundle on $X$ and $\mathcal{F}_{k}$ is a rank $k$ subsheaf of $\mathcal{F}_{n}$. 
We have a natural map of stacks $\operatorname{Bun}_{G} \rightarrow \operatorname{Bun}_{n}$, which sends $\mathcal{F}_{G}$ to the vector bundle $\mathcal{V}_{\mathcal{F}_{G}}$. In addition, we have a map of functors $\widetilde{\operatorname{Bun}}_{P} \rightarrow \operatorname{Bun}_{k, n} \underset{\operatorname{Bun}_{n}}{\times} \operatorname{Bun}_{G}$, that sends a triple $\left(\mathcal{F}_{G}, \mathcal{F}_{M}, \widetilde{\kappa}_{P}\right)$ to $\left(\mathcal{V}_{\mathcal{F}_{M}}^{U(P)} \hookrightarrow \mathcal{V}_{\mathcal{F}_{G}}, \mathcal{F}_{G}\right)$.

It is easy to see that when $\mathcal{V}$ is large enough so that the image of $\mathcal{V}^{*} \otimes \mathcal{V}^{U(P)}$ in $A_{G / U(P)}:=\Gamma\left(G, \mathcal{O}_{G}\right)^{U(P)}$ generates it as an algebra, the above map

$$
\widetilde{\operatorname{Bun}}_{P} \rightarrow \operatorname{Bun}_{k, n} \underset{\operatorname{Bun}_{n}}{\times} \operatorname{Bun}_{G}
$$

is a closed embedding of functors.

This proves the proposition, since the map $\operatorname{Bun}_{k, n} \rightarrow \mathrm{Bun}_{n}$ is known to be representable and proper (cf. [19]).

We will call $\overline{\mathbb{F}_{q}}$-valued points of $\widetilde{\operatorname{Bun}}_{P}$ "enhanced parabolic Drinfeld's structures". A point $\left(\mathcal{F}_{G}, \mathcal{F}_{M}, \widetilde{\kappa}_{P}\right) \in \widetilde{\operatorname{Bun}}_{P}\left(\overline{\mathbb{F}_{q}}\right)$ has singularities at the same finite collection of points as the corresponding simple parabolic Drinfeld's structure, i.e. $\mathfrak{r}_{p}\left(\mathcal{F}_{G}, \mathcal{F}_{M}, \widetilde{\kappa}_{P}\right) \in$ $\overline{\operatorname{Bun}}_{P}\left(\overline{\mathbb{F}_{q}}\right)$. However, the structure of the defect in the enhanced case is more complicated and we will study it in detail in Sect. 6.2.

Proposition 1.3.7. The stack $\mathrm{Bun}_{P}$ is dense in both $\overline{\operatorname{Bun}}_{P}$ and $\widetilde{\operatorname{Bun}}_{P}$.

The proof is postponed until Sect. 6.2.

1.3.8. We will denote by $\widetilde{j}_{P}$ the open embedding $\operatorname{Bun}_{P} \hookrightarrow \widetilde{\operatorname{Bun}}_{P}$ and by $\widetilde{\mathfrak{p}}_{P}$ (resp., $\widetilde{\mathfrak{q}}_{P}$ ) the natural map $\widetilde{\operatorname{Bun}}_{P} \rightarrow \operatorname{Bun}_{G}$ (resp., $\widetilde{\operatorname{Bun}}_{P} \rightarrow \operatorname{Bun}_{M}$ ) given by $\left(\mathcal{F}_{G}, \mathcal{F}_{M}, \widetilde{\kappa}_{P}\right) \mapsto \mathcal{F}_{G}$ and $\left(\mathcal{F}_{G}, \mathcal{F}_{M}, \widetilde{\kappa}_{P}\right) \mapsto \mathcal{F}_{M}$, respectively.

Let us observe now that there is a natural map of stacks $\mathfrak{r}_{P}: \widetilde{\operatorname{Bun}}_{P} \rightarrow \overline{\operatorname{Bun}}_{P}$, which extends the identity map on $\operatorname{Bun}_{P}$ :

In terms of $\left(\mathcal{F}_{G}, \mathcal{F}_{M}, \widetilde{\kappa}_{P}\right)$, it corresponds to the projection $M \rightarrow M /[M, M]$ and the $\operatorname{map} \overline{G / U(P)} \rightarrow \overline{G /[P, P]}$. In terms of the Plücker picture, $\mathfrak{r}_{P}$ "remembrs" only the embeddings $\widetilde{\kappa}_{P}^{\mathcal{V}}$ for $\mathcal{V}=\mathcal{V}_{\mathfrak{F}_{G}}^{\check{\lambda}}, \check{\lambda} \in \check{\Lambda}_{G, P} \cap \Lambda_{G}^{+}$(in this case the representation $\mathcal{U}^{\check{\lambda}}$ of $M$ factors through $M \rightarrow M /[M, M])$.

Since $\widetilde{\mathfrak{p}}_{P}=\overline{\mathfrak{p}}_{P} \circ \mathfrak{r}_{P}$, the map $\mathfrak{r}_{P}$ is representable and proper. We will show in Sect. 6.2 that its fibers can be identified with closed subvarieties of the affine Grassmannian of the group $M$.

\section{MAin RESUlts ON EISENSTEIN SERIES}

2.1. Principal Eisenstein series. First, we will discuss the principal Eisenstein series functor that maps the category $\operatorname{Sh}\left(\operatorname{Bun}_{T}\right)$ to $\operatorname{Sh}\left(\operatorname{Bun}_{G}\right)$.

2.1.1. Let $\overline{\mathfrak{q}}^{! *}$ denote the functor $\mathrm{Sh}\left(\operatorname{Bun}_{T}\right) \rightarrow \operatorname{Sh}\left(\overline{\operatorname{Bun}}_{B}\right)$ given by

$$
\mathcal{S} \rightarrow \mathrm{IC}_{\overline{\overline{\mathrm{Bun}}_{B}}} \otimes \overline{\mathfrak{q}}^{*}(\mathcal{S}) \otimes\left(\overline{\mathbb{Q}_{\ell}}\left(\frac{1}{2}\right)[1]\right)^{\otimes-\operatorname{dim}\left(\operatorname{Bun}_{T}\right)} .
$$


Analogously, we define the functor $\mathfrak{q}^{! *}:=j^{*} \circ \overline{\mathfrak{q}}^{!^{*}}: \operatorname{Sh}\left(\operatorname{Bun}_{T}\right) \rightarrow \operatorname{Sh}\left(\operatorname{Bun}_{B}\right)$. Note, that since the map $\mathfrak{q}$ is smooth, the latter functor is an ordinary pull-back up to a cohomological shift and Tate's twist.

Theorem 2.1.2. (a) The functor $\overline{\mathfrak{q}}^{! *}$ is exact and commutes with Verdier duality.

(b) If $\mathcal{S}$ is perverse, we have: $\overline{\mathfrak{q}}^{! *}(\mathcal{S}) \simeq j_{! *} \circ \mathfrak{q}^{! *}(\mathcal{S})$.

We define the functor $\operatorname{Eis}_{T}^{G}$ (or simply Eis) $\operatorname{Sh}\left(\operatorname{Bun}_{T}\right) \rightarrow \operatorname{Sh}\left(\operatorname{Bun}_{G}\right)$ by:

$$
\operatorname{Eis}(\mathcal{S}):=\overline{\mathfrak{p}}_{!} \circ \overline{\mathfrak{q}}^{! *}(\mathcal{S})
$$

Corollary 2.1.3. The functor Eis commutes with Verdier duality and maps pure complexes to pure ones.

2.1.4. Let $\mathcal{H}_{G}$ be the Hecke stack, i.e. an $S$-point of $\mathcal{H}_{G}$ is a quadruple: $\left(x, \mathcal{F}_{G}, \mathcal{F}_{G}^{\prime}, \beta\right)$, where $x \in \operatorname{Hom}(S, X), \mathcal{F}_{G}$ and $\mathcal{F}_{G}^{\prime}$ are $S$-points of $\operatorname{Bun}_{G}$ (i.e. $G$-bundles on $X \times S$ ) and $\beta$ is an isomorphism

$$
\beta:\left.\left.\mathcal{F}_{G}\right|_{X \times S-\Gamma_{x}} \rightarrow \mathcal{F}_{G}^{\prime}\right|_{X \times S-\Gamma_{x}}
$$

where $\Gamma_{x} \subset X \times S$ is the graph of the map $x: S \rightarrow X$.

We have three projections: $\pi: \mathcal{H}_{G} \rightarrow X, h_{G}: \mathcal{H}_{G} \rightarrow \operatorname{Bun}_{G}$ and $h_{G}^{\overleftarrow{G}}: \mathcal{H}_{G} \rightarrow \operatorname{Bun}_{G}$

$$
\pi\left(x, \mathcal{F}_{G}, \mathcal{F}_{G}^{\prime}, \beta\right)=x ; h_{G}\left(x, \mathcal{F}_{G}, \mathcal{F}_{G}^{\prime}, \beta\right)=\mathcal{F}_{G}^{\prime} ; h_{G}^{\overleftarrow{ }}\left(x, \mathcal{F}_{G}, \mathcal{F}_{G}^{\prime}, \beta\right)=\mathcal{F}_{G}
$$

i.e. we have a diagram:

$$
\operatorname{Bun}_{G} \times X \stackrel{h_{G}^{\leftarrow} \times \pi}{\longleftarrow} \mathcal{H}_{G} \stackrel{h_{G}}{\longrightarrow} \operatorname{Bun}_{G} .
$$

For a dominant coweight $\lambda$ of $G$ we introduce a closed substack $\overline{\mathcal{H}}_{G}^{\lambda}$ of $\mathcal{H}_{G}$ as follows: By definition, a quadruple $\left(x, \mathcal{F}_{G}, \mathcal{F}_{G}^{\prime}, \beta\right)$ belongs to $\overline{\mathcal{H}}_{G}^{\lambda}$ if and only if for every $G$ module $\mathcal{V}$ such all its weights are $\leq \check{\lambda}$ for some $\check{\lambda} \in \check{\Lambda}_{G}^{+}$,

$$
\mathcal{V}_{\mathcal{F}_{G}}\left(-\langle\lambda, \check{\lambda}\rangle \cdot \Gamma_{x}\right) \subset \mathcal{V}_{\mathcal{F}_{G}^{\prime}} \subset \mathcal{V}_{\mathcal{F}_{G}}\left(-\left\langle\mathrm{w}_{0}(\lambda), \check{\lambda}\right\rangle \cdot \Gamma_{x}\right)
$$

(Of course, the second inclusion is a corollary of the first one for the dual representation $\mathcal{V}^{*}$.) As we will see later, both maps $h_{G}^{\leftarrow} \times \pi$ and $h_{G} \times \pi$ when restricted to $\overline{\mathcal{H}}_{G}^{\lambda}$ are proper and, in addition, are locally-trivial fibrations in the smooth topology (cf. Sect. 0.4).

For instance, when $G=T$, the projection $h_{G}^{\leftarrow} \times \pi$ defines an isomorphism $\overline{\mathcal{H}}_{T}^{\mu} \rightarrow$ $\operatorname{Bun}_{T} \times X$ and the composition $\operatorname{Bun}_{T} \times X \simeq \overline{\mathcal{H}}_{T}^{\mu} \stackrel{h_{\vec{T}}}{\longrightarrow} \operatorname{Bun}_{T}$ sends

$$
\left(\mathcal{F}_{T}, x\right) \in \operatorname{Bun}_{T} \times X \longrightarrow \mathcal{F}_{T}^{\prime}:=\mathcal{F}_{T}(-\mu \cdot x) \in \operatorname{Bun}_{T} .
$$

Each $\overline{\mathcal{H}}_{G}^{\lambda}$ is an algebraic stack locally of finite type and let $\mathrm{IC}_{\overline{\mathcal{F}}_{G}^{\lambda}}$ denote the intersection cohomology sheaf on it. We define the Hecke functor $H_{G}^{\lambda}: \operatorname{Sh}\left(\operatorname{Bun}_{G}\right) \rightarrow$ $\operatorname{Sh}\left(\operatorname{Bun}_{G} \times X\right)$ by

$$
\mathcal{S} \rightarrow\left(h_{G}^{\leftarrow} \times \pi\right)_{!}\left(h_{G}^{*}(\mathcal{S}) \otimes \mathrm{IC}_{\overline{\mathcal{H}}_{G}^{\lambda}}\right) \otimes\left(\overline{\mathbb{Q}_{\ell}}\left(\frac{1}{2}\right)[1]\right)^{\otimes-\operatorname{dim}\left(\operatorname{Bun}_{G}\right)}
$$

As the map $h_{G}^{\overleftarrow{ }} \times \pi: \overline{\mathcal{H}}_{G}^{\lambda} \rightarrow \operatorname{Bun}_{G}$ is proper and $h_{G}: \overline{\mathcal{H}}_{G}^{\lambda} \rightarrow \operatorname{Bun}_{G}$ is a fibration, the functor $H_{G}^{\lambda}$ commutes with Verdier duality. 
Recall that for $\lambda \in \Lambda_{G}^{+}, V^{\lambda}$ denotes the corresponding irreducible representation of the Langlands dual group $\breve{G}$ and for a coweight $\mu, V^{\lambda}(\mu)$ is the corresponding weight subspace of $V^{\lambda}$.

Theorem 2.1.5. For every $\lambda \in \Lambda_{G}^{+}$and $\mathcal{S} \in \operatorname{Sh}\left(\operatorname{Bun}_{T}\right)$ we have a functorial isomorphism:

$$
H_{G}^{\lambda} \circ \operatorname{Eis}(\mathcal{S}) \simeq \bigoplus_{\mu \in \Lambda}(\operatorname{Eis} \otimes \mathrm{id}) \circ H_{T}^{\mu}(\mathcal{S}) \otimes V^{\lambda}(\mu),
$$

where we used the notation Eis $\square$ id for the corresponding functor $\operatorname{Sh}\left(\operatorname{Bun}_{T} \times X\right) \rightarrow$ $\operatorname{Sh}\left(\operatorname{Bun}_{G} \times X\right)$, and for $\mu \in \Lambda, H_{T}^{\mu}$ denotes the corresponding Hecke functor for the group $T$.

2.1.6. The Hecke functors can be composed in the following way: If $\lambda_{1}$ and $\lambda_{2}$ are two elements of $\Lambda_{G}^{+}$, we define the functor

$$
H_{G}^{\lambda_{1}} \star H_{G}^{\lambda_{2}}: \operatorname{Sh}\left(\operatorname{Bun}_{G}\right) \rightarrow \operatorname{Sh}\left(\operatorname{Bun}_{G} \times X\right)
$$

by the rule:

$$
H_{G}^{\lambda_{1}} \star H_{G}^{\lambda_{2}}(\mathcal{S})=\left(\operatorname{id} \otimes \Delta_{X}^{*}\right)\left(\left(H_{G}^{\lambda_{1}} \otimes \mathrm{id}\right) \circ H_{G}^{\lambda_{2}}(\mathcal{S})\right) \otimes\left(\overline{\mathbb{Q}_{\ell}}\left(\frac{1}{2}\right)[1]\right)^{\otimes-1}
$$

where $\Delta_{X}: X \rightarrow X \times X$ denotes the diagonal embedding.

It is well-known ([3]) that there exists a canonical isomorphism of functors:

$$
H_{G}^{\lambda_{1}} \star H_{G}^{\lambda_{2}}(\mathcal{S}) \simeq \underset{\lambda \in \Lambda_{G}^{+}}{\oplus} H_{G}^{\lambda}(\mathcal{S}) \otimes \operatorname{Hom}_{\check{G}}\left(V^{\lambda}, V^{\lambda_{1}} \otimes V^{\lambda_{2}}\right)
$$

An additional property of the isomorphism of Theorem 2.1.5 is that for $\mathcal{S} \in \operatorname{Sh}\left(\operatorname{Bun}_{T}\right)$ the two isomorphisms:

$$
\begin{aligned}
& \left(H_{G}^{\lambda_{1}} \star H_{G}^{\lambda_{2}}\right) \circ \operatorname{Eis}(\mathcal{S}) \simeq \underset{\mu_{1}, \mu_{2}}{\oplus}(\operatorname{Eis} \otimes \mathrm{id}) \circ\left(H_{T}^{\mu_{1}} \star H_{T}^{\mu_{2}}\right)(\mathcal{S}) \otimes V^{\lambda_{1}}\left(\mu_{1}\right) \otimes V^{\lambda_{2}}\left(\mu_{2}\right) \simeq \\
& \simeq \underset{\lambda}{\oplus} \underset{\mu}{\oplus}(\operatorname{Eis} \otimes \mathrm{id}) \circ H_{T}^{\mu}(\mathcal{S}) \otimes \operatorname{Hom}_{\breve{G}}\left(V^{\lambda}, V^{\lambda_{1}} \otimes V^{\lambda_{2}}\right) \otimes V^{\lambda}(\mu)
\end{aligned}
$$

and

$$
\begin{aligned}
& H_{G}^{\lambda_{1}} \star H_{G}^{\lambda_{2}} \circ \operatorname{Eis}(\mathcal{S}) \simeq \underset{\lambda}{\oplus} H_{G}^{\lambda} \circ \operatorname{Eis}(\mathcal{S}) \otimes \operatorname{Hom}_{\check{G}}\left(V^{\lambda}, V^{\lambda_{1}} \otimes V^{\lambda_{2}}\right) \simeq \\
& \simeq \underset{\lambda}{\oplus} \bigoplus_{\mu}(\operatorname{Eis} \otimes \mathrm{id}) \circ H_{T}^{\nu^{\prime}}(\mathcal{S}) \otimes \operatorname{Hom}_{\breve{G}}\left(V^{\lambda}, V^{\lambda_{1}} \otimes V^{\lambda_{2}}\right) \otimes V^{\lambda}(\mu)
\end{aligned}
$$

coincide.

2.1.7. For a coroot $\alpha \in \Delta^{+}$of $G$, consider the corresponding projection $T \rightarrow T / \mathbb{G}_{m}$. This map of groups gives rise to a map of stacks

$$
\mathfrak{f}^{\alpha}: \operatorname{Bun}_{T} \rightarrow \operatorname{Bun}_{T / \mathbb{G}_{m}} .
$$

We will call a sheaf $\mathcal{S}$ on $\mathrm{Bun}_{T}$ regular if

$$
\mathfrak{f}_{!}^{\alpha}(\mathcal{S})=0, \quad \forall \alpha \in \Delta^{+}
$$

(this definition makes sense even though the map $\mathfrak{f}^{\alpha}$ is non-representable). We let $\mathrm{Sh}\left(\operatorname{Bun}_{T}\right)^{r e g} \subset \operatorname{Sh}\left(\operatorname{Bun}_{T}\right)$ denote the full triangulated subcategory of regular sheaves. 
Observe now that the action of the Weyl group on $T$ gives rise to a $W$-action on the stack $\operatorname{Bun}_{T}: \mathcal{F}_{T} \rightarrow \mathcal{F}_{T}^{\mathrm{w}}$. We define a twisted $W$-action on $\operatorname{Bun}_{T}$ as follows:

For an element $\lambda \in \Lambda$, consider the $T$-bundle $\Omega^{\lambda}$ on $X$ induced from the canonical line bundle on $X$ by means of the homomorphism $\mathbb{G}_{m} \rightarrow T$ corresponding to $\lambda$.

We set

$$
\mathrm{w} \cdot \mathcal{F}_{T}=\mathcal{F}_{T}^{\mathrm{w}} \otimes \Omega^{\mathrm{w}(\rho)-\rho} .
$$

Similarly, for $\mathrm{w} \in W$ we will denote by $\mathcal{S} \rightarrow \mathrm{w} \cdot \mathcal{S}$ the direct image functor $\operatorname{Sh}\left(\operatorname{Bun}_{T}\right) \rightarrow$ $\mathrm{Sh}\left(\operatorname{Bun}_{T}\right)$ corresponding to the new action of $\mathrm{w}$ on $\mathrm{Bun}_{T}$.

It is easy to see that for every $\mathrm{w} \in W$ the functor $\mathcal{S} \rightarrow \mathrm{w} \cdot \mathcal{S}$ preserves the subcategory $\operatorname{Sh}\left(\operatorname{Bun}_{T}\right)^{r e g} \subset \operatorname{Sh}\left(\operatorname{Bun}_{T}\right)$. Let $N(\check{T})$ denote the normalizer of $\check{T}$ in $\check{G}$.

Theorem 2.1.8. For each $\mathrm{w} \in W$ with a choice of a lift to an element $\widetilde{\mathrm{w}} \in N(\check{T}) \subset \check{G}$, there exists a functorial isomorphism

$$
\operatorname{Eis}(\mathrm{w} \cdot \mathcal{S}) \stackrel{f \cdot e q}{\longrightarrow} \operatorname{Eis}(\mathcal{S}), \mathcal{S} \in \operatorname{Sh}\left(\operatorname{Bun}_{T}\right)^{r e g} .
$$

2.1.9. The isomorphisms of Theorem 2.1.8 and Theorem 2.1.5 are compatible with the following way: the two isomorphisms

$$
H_{G}^{\lambda} \circ \operatorname{Eis}(\mathrm{w} \cdot \mathcal{S}) \rightrightarrows \underset{\nu \in \Lambda}{\oplus}(\operatorname{Eis} \otimes \mathrm{id}) \circ H_{T}^{\nu}(\mathcal{S}) \otimes V^{\lambda}(\mathrm{w}(\nu))
$$

(see below) coincide, where the first isomorphism is simply

$$
\begin{aligned}
& H_{G}^{\lambda} \circ \operatorname{Eis}(\mathrm{w} \cdot \mathcal{S}) \stackrel{f . e q}{\longrightarrow} H_{G}^{\lambda} \circ \operatorname{Eis}(\mathcal{S}) \simeq \underset{\nu \in \Lambda}{\oplus}(\text { Eis } \otimes \mathrm{id}) \circ H_{T}^{\nu}(\mathcal{S}) \otimes V^{\lambda}(\nu) \simeq \\
& \underset{\nu \in \Lambda}{\oplus}(\operatorname{Eis} \otimes \mathrm{id}) \circ H_{T}^{\nu}(\mathcal{S}) \otimes V^{\lambda}(\mathrm{w}(\nu)),
\end{aligned}
$$

(the last arrow comes from the map $V^{\lambda}(\nu) \rightarrow V^{\lambda}(\mathrm{w}(\nu))$ given by the action of $\widetilde{\mathrm{w}} \in \check{G}$ ), and the second isomorphism is the composition:

$$
\begin{aligned}
& H_{G}^{\lambda} \circ \operatorname{Eis}(\mathrm{w} \cdot \mathcal{S}) \simeq \underset{\nu \in \Lambda}{\oplus}(\text { Eis } \otimes \mathrm{id}) \circ H_{T}^{\nu}(\mathrm{w} \cdot \mathcal{S}) \otimes V^{\lambda}(\nu) \simeq \\
& \underset{\nu \in \Lambda}{\oplus}(\operatorname{Eis} \otimes \mathrm{id}) \circ(\mathrm{w} \cdot \otimes \mathrm{id}) \circ H_{T}^{\mathrm{w}^{-1}(\nu)}(\mathcal{S}) \otimes V^{\lambda}(\nu) \stackrel{f \cdot e q}{\longrightarrow} \underset{\nu \in \Lambda}{\oplus}(\text { Eis } \otimes \mathrm{id}) \circ H_{T}^{\mathrm{w}^{-1}(\nu)}(\mathcal{S}) \otimes V^{\lambda}(\nu) \\
& \simeq \underset{\nu^{\prime} \in \Lambda}{\oplus}(\text { Eis } \otimes \mathrm{id}) \circ H_{T}^{\nu^{\prime}}(\mathcal{S}) \otimes V^{\lambda}\left(\mathrm{w}\left(\nu^{\prime}\right)\right),
\end{aligned}
$$

where the second arrow used the isomorphism $H_{T}^{\nu}(\mathrm{w} \cdot \mathcal{S}) \simeq(\mathrm{w} \cdot \bigotimes \mathrm{id}) \circ H_{T}^{\mathrm{w}^{-1}(\nu)}(\mathcal{S})$, which holds for any $\mathcal{S} \in \operatorname{Sh}\left(\operatorname{Bun}_{T}\right)$.

Remark. Our construction of the isomorphism of functors

$$
\operatorname{Eis}(\mathrm{w} \cdot \mathcal{S}) \stackrel{f \cdot e q}{\longrightarrow} \operatorname{Eis}(\mathcal{S}): \operatorname{Sh}\left(\operatorname{Bun}_{T}\right)^{r e g} \rightarrow \operatorname{Sh}\left(\operatorname{Bun}_{G}\right)
$$

of Theorem 2.1.8 involves an additional choice of a representation of $\mathrm{w}$ as a product of simple reflections. At the moment, we can prove that this isomorphism is independent of such a representation only when $G$ is simply-laced.

Conjecture 2.1.10. For $\mathcal{S} \in \operatorname{Sh}\left(\operatorname{Bun}_{T}\right)^{r e g} \cap \operatorname{Perv}\left(\operatorname{Bun}_{T}\right)$, the object $\operatorname{Eis}(\mathcal{S})$ is a perverse sheaf. Moreover, if $\mathcal{S}$ is irreducible, then so is $\operatorname{Eis}(\mathcal{S})$. 
Remark. When $G$ has semi-simle rank 1, or when $G=G L(n)$ and $\mathcal{S}$ is a local system, this conjecture is in fact a theorem (cf. 14]).

2.2. Relation with the classical theory. Let now $E_{\breve{T}}$ be a $\check{T}$-local system on $X$. For $\lambda \in \Lambda$ we will denote by $E_{\breve{T}}^{\lambda}$ the 1 -dimensional local system on $X$ induced from $E_{\check{T}}$ by means of the homomorphism $\check{T} \stackrel{\lambda}{\rightarrow} \mathbb{G}_{m}$.

2.2.1. The (geometric) abelian class field theory produces from $E_{\check{T}}$ a 1-dimensional local system, which we will denote by $\operatorname{Aut}_{E_{\breve{T}}}^{\prime}$ on $\operatorname{Bun}_{T}$, whose fiber at $\mathcal{F}_{T}=\mathcal{F}_{T}^{0}\left(\sum_{i} \mu_{i} \cdot x_{i}\right)$ is $\underset{i}{\otimes}\left(E_{\breve{T}}^{-\mu_{i}}\right)_{x_{i}}$, where the subscript " $x_{i}$ " means "fiber at $x_{i}$ ".

Let $\sqrt{\left(\text { Aut }_{E_{\check{T}}}^{\prime}\right)_{\Omega_{X}^{2 \rho}}}$ be a 1-dimensional vector space such that $\left(\sqrt{\left(\text { Aut }_{E_{\breve{T}}^{\prime}}\right)_{\Omega_{X}^{2 \rho}}}\right)^{\otimes 2} \simeq$ $\left(\text { Aut }_{E_{\breve{T}}}^{\prime}\right)_{\Omega_{X}^{2 \rho}}$. We define a perverse sheaf $\mathrm{Aut}_{E_{\breve{T}}}$ on $\mathrm{Bun}_{T}$ as a tensor product of $\mathrm{Aut}_{E_{\breve{T}}}^{\prime}$ with the 1-dimensional vector space $\sqrt{\left(\operatorname{Aut}_{E_{\breve{T}}}^{\prime}\right)_{\Omega_{X}^{2 \rho}}} \otimes\left(\overline{\mathbb{Q}_{\ell}}\left(\frac{1}{2}\right)\right)^{\otimes(g-1) \operatorname{dim}(T)}$, placed in the cohomological degree $(1-g) \cdot \operatorname{dim}(T)$.

By construction, the perverse sheaf Aut $_{E_{\breve{T}}}$ has the following property with respect to the twisted $W$-action on $\operatorname{Bun}_{T}$ :

$$
\mathrm{w} \cdot \operatorname{Aut}_{E_{\breve{T}}} \simeq \operatorname{Aut}_{E_{\breve{T}}^{\mathrm{w}}}
$$

where $E_{\check{T}}^{\mathrm{w}}$ is a $\check{T}$-local system induced from $E_{\breve{T}}$ by means of $\check{T} \stackrel{\mathrm{w}}{\longrightarrow} \check{T}$.

It is clear from the definitions that the perverse sheaf Aut $_{E_{\breve{T}}}$ is a Hecke eigen-sheaf on $\operatorname{Bun}_{T}$ with respect to $E_{\breve{T}}$, i.e. for $\lambda \in \Lambda_{T}$, we have:

$$
H_{T}^{\mu}\left(\operatorname{Aut}_{E_{\breve{T}}}\right) \simeq \operatorname{Aut}_{E_{\check{T}}} \otimes E_{\check{T}}^{\mu} \otimes \overline{\mathbb{Q}_{\ell}}\left(\frac{1}{2}\right)[1] .
$$

Let $E_{\check{G}}$ denote the $\check{G}$-local system on $X$ induced from $E_{\check{T}}$ by means of the canonical embedding $\check{T} \hookrightarrow \check{G}$. Theorem 2.1 .5 yields the following result:

Theorem 2.2.2. The object $\operatorname{Eis}\left(\mathrm{Aut}_{E_{\overparen{T}}}\right) \in \operatorname{Sh}\left(\operatorname{Bun}_{G}\right)$ is a Hecke eigen-sheaf on $\operatorname{Bun}_{G}$ with respect to $E_{\check{G}}$, i.e. for each $\lambda \in \Lambda_{G}^{+}$we have:

$$
H_{G}^{\lambda} \circ \operatorname{Eis}\left(\operatorname{Aut}_{E_{\check{T}}}\right) \simeq \operatorname{Eis}\left(\operatorname{Aut}_{E_{\breve{T}}}\right) \otimes V_{E_{\breve{G}}}^{\lambda} \otimes \overline{\mathbb{Q}_{\ell}}\left(\frac{1}{2}\right)[1],
$$

where $V_{E_{\breve{G}}}^{\lambda}$ is a local system on $X$ associated to $E_{\breve{G}}$ and the $\check{G}$-module $V^{\lambda}$.

2.2.3. Assume now that the $\check{T}$-local system $E_{\check{T}}$ is regular, i.e. that for every $\alpha \in \Delta^{+}$ the 1-dimesnional local system $E_{\breve{T}}^{\alpha}$ on $X$ is non-trivial. This condition is equivalent to the fact that the perverse sheaf $\mathrm{Aut}_{E_{\check{T}}}$ belongs to $\mathrm{Sh}\left(\mathrm{Bun}_{T}\right)^{r e g}$.

For $\mathrm{w} \in W$ let us choose a representative $\widetilde{\mathrm{w}} \in N(\check{T})$. Note that this gives an isomorphism $E_{\check{G}} \simeq \operatorname{Ind}_{\check{T}}^{\check{G}}\left(E_{\check{T}}^{\mathrm{w}}\right)$. By applying Theorem 2.1.8, we obtain the following assertion: 
Theorem 2.2.4. For $\widetilde{\mathrm{w}} \in N(\check{T})$ and $E_{\breve{G}}$ as above there is an isomorphism (functional equation):

Moreover, the diagram

$$
\operatorname{Eis}\left(\operatorname{Aut}_{E_{\check{T}}}\right) \stackrel{f \cdot e q}{\simeq} \operatorname{Eis}\left(\operatorname{Aut}_{E_{\tilde{T}}^{\mathrm{w}}}\right)
$$

$$
\begin{aligned}
H_{G}^{\lambda} \circ \operatorname{Eis}\left(\operatorname{Aut}_{E_{\breve{T}}}\right) & \operatorname{Eis}\left(\operatorname{Aut}_{E_{\breve{T}}}\right) \otimes V_{E_{\check{G}}}^{\lambda} \otimes \overline{\mathbb{Q}_{\ell}}\left(\frac{1}{2}\right)[1] \\
H_{G}^{\lambda}(f . e q) & f . e q \unrhd \mathrm{id} \\
H_{G}^{\lambda} \circ \operatorname{Eis}\left(\operatorname{Aut}_{E_{\breve{T}}^{\mathrm{w}}}\right) & \longrightarrow \operatorname{Eis}\left(\operatorname{Aut}_{E_{\breve{T}}^{\mathrm{w}}}\right) \otimes V_{E_{\breve{G}}}^{\lambda} \otimes \overline{\mathbb{Q}_{\ell}}\left(\frac{1}{2}\right)[1]
\end{aligned}
$$

commutes.

The stack $\mathrm{Bun}_{T}$ splits into connected components $\mathrm{Bun}_{T}^{\mu}$, numbered by the elements of $\Lambda$ : by definition $\mathcal{F}_{T}^{0}\left(\Sigma \mu_{i} \cdot x_{i}\right) \in \operatorname{Bun}_{T}^{-\mu}$ if $\Sigma \mu_{i}=\mu$. Let Aut $_{E_{\breve{T}}}^{\mu}$ be the direct summand of Aut $_{E_{\breve{T}}}$ concentrated on $\mathrm{Bun}_{T}^{\mu}$.

Theorem 2.2.4 implies the following assertion, which is hard to see directly:

Corollary 2.2.5. Let $E_{\check{T}}$ be a regular $\check{T}$-local system and let $U \subset \operatorname{Bun}_{G}$ be an open substack of finite type. Then the sheaves $\left.\operatorname{Eis}\left(\mathrm{Aut}_{E_{\widetilde{T}}}^{\mu}\right)\right|_{U}$ are zero except for finitely many $\mu \in \Lambda$.

Proof. The proof is a combination of Theorem 2.2.4 and of the following (obvious) statement:

Lemma 2.2.6. Let $U \subset \operatorname{Bun}_{G}$ be an open sub-stack of finite type. Then the set of $\mu \in \Lambda$ for which the intersection $\overline{\mathfrak{p}}^{-1}(U) \cap \overline{\mathfrak{q}}^{-1}\left(\operatorname{Bun}_{T}^{\mu}\right)$ is non-empty has the form

$$
\mu \geq \mu^{\prime}
$$

where $\mu^{\prime}$ is some fixed element of $\Lambda$.

2.2.7. Theorem 2.2.4 implies the following special case of the Langlands' conjecture:

Let $\mathcal{K}$ be the field of rational functions on $X$ and let $G_{\mathbb{A}}$, (resp., $G_{\mathbb{O}} \subset G_{\mathbb{A}}, G_{\mathcal{K}} \subset G_{\mathbb{A}}$ ) be the corresponding adele group (resp., the group of integral points of $G_{\mathbb{A}}$, the group of rational points of $\left.G_{\mathbb{A}}\right)$. Recall that the double quotient $G_{\mathbb{O}} \backslash G_{\mathbb{A}} / G_{\mathcal{K}}$ identifies with the set of isomorphism classes of objects of the category $\operatorname{Bun}_{G}\left(\mathbb{F}_{q}\right)$, i.e. with the set of isomorphism classes of $G$-bundles over $X$.

Theorem 2.2.8. Let $E_{\breve{G}}$ be an irreducible $\breve{G}$-local system on $X$, such that if we "forget" the Weil structure on $E_{\breve{G}}$, it admits a reduction to $\check{T} \subset \check{G}$. Then there exists a spherical automorphic function on $G_{\mathbb{A}}$ whose Langlands' parameters correspond to $E_{\breve{G}}$.

Proof. Let $\bar{X} \simeq X \underset{\operatorname{Spec}\left(\mathbb{F}_{q}\right)}{\times} \operatorname{Spec}\left(\overline{\mathbb{F}_{q}}\right)$ and let $\bar{E}_{\breve{G}}$ denote $E_{\breve{G}}$, viewed as a local system just on $\bar{X}$. Let $F r$ denote the Frobenius acting on $\bar{X}$ and on $\overline{\operatorname{Bun}_{G}}:=\operatorname{Bun}_{G} \underset{\operatorname{Spec}\left(\mathbb{F}_{q}\right)}{\times} \operatorname{Spec}\left(\overline{\mathbb{F}_{q}}\right)$.

To construct the sought-for automorphic function it is sufficient to produce an object Aut $_{E_{\bar{G}}} \in \operatorname{Sh}\left(\overline{\operatorname{Bun}_{G}}\right)$ together with a Weil structure on it, which is a Hecke eigen-sheaf 
with respect to $\bar{E}_{\breve{G}}$ in a $F r$-compatible way. In other words, we need that there exists an isomorphism:

$$
\operatorname{Fr}^{*}\left(\operatorname{Aut}_{\bar{E}_{\check{G}}}\right) \rightarrow \operatorname{Aut}_{\bar{E}_{\check{G}}}
$$

such that for each $\lambda \in \Lambda_{G}^{+}$the diagram

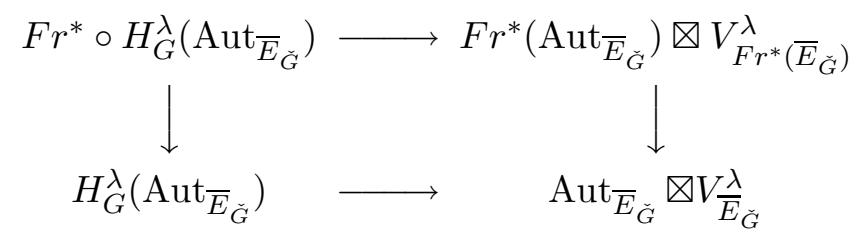

is commutative.

Let $\bar{E}_{\check{T}}$ be a reduction of the $\breve{G}$-local system $\bar{E}_{\breve{G}}$ over $\bar{X}$ to $\check{T} \subset \check{G}$ (such a reduction exists according to our assumtion on $E_{\breve{G}}$ ). We have now the following assertion: 円

Proposition 2.2.9. (a) Let $\bar{E}_{\breve{T}}$ be as above and assume (as in the formulation of Theorem 2.2.8) that $E_{\breve{G}}$ is irreducible. Then the $\check{T}$-local system $\bar{E}_{\breve{T}}$ is regular.

(b) There exists an element $\mathrm{w} \in W$ and its lift $\widetilde{\mathrm{w}} \in N(\check{T})$ such that $\operatorname{Fr}^{*}\left(\bar{E}_{\breve{T}}\right) \simeq \bar{E}_{\breve{T}}^{\mathrm{w}}$ and such the identification

$$
\operatorname{Ind}_{\check{T}}^{\check{G}}\left(\bar{E}_{\check{T}}\right) \simeq \bar{E}_{\check{G}} \simeq F r^{*}\left(\bar{E}_{\check{G}}\right) \simeq \operatorname{Ind}_{\breve{T}}^{\check{G}}\left(F r^{*}\left(\bar{E}_{\check{T}}\right)\right) \simeq \operatorname{Ind}_{\check{T}}^{\check{G}}\left(\bar{E}_{\breve{T}}^{\mathrm{w}}\right)
$$

is induced by $\widetilde{\mathrm{w}}$.

We define now the object Aut $\bar{E}_{\breve{G}}$ as $\operatorname{Eis}\left(\right.$ Aut $\left._{\bar{E}_{\breve{T}}}\right)$. Using Theorem 2.2.4 and Proposition 2.2 .9 we construct an isomorphism $F r^{*}\left(\operatorname{Eis}\left(\operatorname{Aut}_{\bar{E}_{\overparen{T}}}\right)\right) \simeq \operatorname{Eis}\left(\operatorname{Aut}_{\bar{E}_{\breve{T}}}\right)$ that corresponds to the above $\widetilde{\mathrm{w}}$. Theorem 2.2.4 guarantees that Aut $\bar{E}_{\breve{G}}$ possesses all the required properties.

2.2.10. Let $E_{\check{T}}$ be again an arbitrary (not necessarily regular) $\check{T}$-local system on $X$. We will now investigate the connection between the function on $G_{\mathbb{O}} \backslash G_{\mathbb{A}} / G_{\mathcal{K}} \simeq$ $\operatorname{Bun}_{G}\left(\mathbb{F}_{q}\right)$ corresponding to $\operatorname{Eis}\left(\operatorname{Aut}_{E_{\check{T}}}\right) \in \operatorname{Sh}\left(\operatorname{Bun}_{G}\right)$ and the classical Eisenstein series constructed starting with $E_{\breve{T}}$.

For $E_{\breve{T}}$ as above and $\mu \in \Lambda$ consider the object $\operatorname{Eis}^{\prime}\left(\right.$ Aut $\left._{E_{\check{T}}}^{\mu}\right)$ defined as

$$
\mathfrak{p}_{!} \circ \mathfrak{q}^{! *}\left(\mathrm{Aut}_{E_{\check{T}}}^{\mu}\right) \text {. }
$$

We let Funct $\left(\operatorname{Eis}\left(\operatorname{Aut}_{E_{\breve{T}}}^{\mu}\right)\right)$ (resp., Funct $\left(\operatorname{Eis}^{\prime}\left(\operatorname{Aut}_{E_{\breve{T}}}^{\mu}\right)\right)$ ) denote the functions on the set $\operatorname{Bun}_{G}\left(\mathbb{F}_{q}\right)$ corresponding to $\operatorname{Eis}\left(\operatorname{Aut}_{E_{\check{T}}}\right)$ and $\operatorname{Eis}^{\prime}\left(\operatorname{Aut}_{E_{\check{T}}}\right)$, respectively.

The following result is a corollary of a description of stalks of the intersection cohomology sheaf on $\overline{\mathrm{Bun}}_{B}$ (Theorem 6.1.6): 9

\footnotetext{
${ }^{1}$ We are obliged to G. Prasad who has explained to us the proof of this result. He has moreover authorized us to reproduce his argument, which we shall do in the Appendix.

${ }^{2}$ The proof of Theorem 6.1.6 will be given in a forthcoming paper []. For the case $X=\mathbb{P}^{1}$ this result is proved in [12]. Note, however, that all the results of this paper except for Theorem 2.2.11 and Theorem 2.2.12 are independent of Theorem 6.1.6.
} 
Theorem 2.2.11. The function Funct $\left(\operatorname{Eis}\left(\right.\right.$ Aut $\left.\left._{E_{\tilde{T}}}^{\mu}\right)\right)$ equals

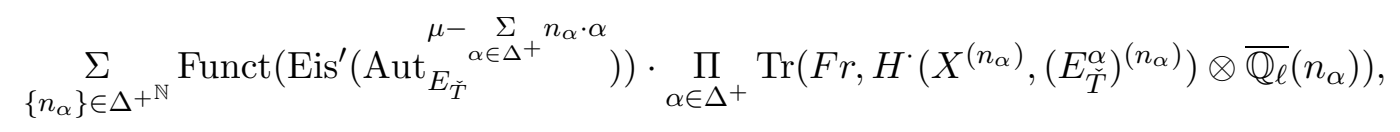

where $X^{(m)}$ denotes the $m$-th symmetric power of $X$ and for a local system $E, E^{(m)}$ denotes the $m$-th symmetric power of $E$.

We will now reformulate Theorem 2.2.11 by encoding the information contained in it into a generating series.

Consider the group-ring $\overline{\mathbb{Q}_{\ell}}[\Lambda]$, which is the same, of course, as the ring of functions on the torus $\check{T}$; for $\mu \in \Lambda$ we shall denote by $t^{\mu}$ the corresponding element of $\overline{\mathbb{Q}_{\ell}}[\Lambda]$. We will form a completed ring $\widehat{\overline{\mathbb{Q}_{\ell}}[\Lambda]}$ by allowing infinite expressions of the form

$$
\sum_{\mu} a_{\mu} \cdot t^{\mu}
$$

if $\mu$ runs over a sub-set of $\Lambda$ of the form $\mu \geq \mu^{\prime}$, where $\mu^{\prime}$ is some fixed element of $\Lambda$.

The classical Eisenstein series can be thought of as a function on $\operatorname{Bun}_{G}\left(\mathbb{F}_{q}\right)$ with values in $\overline{\overline{\mathbb{Q}_{\ell}}[\Lambda]}$, equal to

$$
\operatorname{Eis}_{c l}\left(\operatorname{Aut}_{E_{\check{T}}}\right)(t):=\sum_{\mu \in \Lambda} \operatorname{Funct}\left(\operatorname{Eis}^{\prime}\left(\operatorname{Aut}_{E_{\check{T}}}^{\mu}\right)\right) \cdot t^{\mu} .
$$

Similarly, consider the "modified" Eisenstein series

$$
\operatorname{Eis}_{\text {mod }}\left(\operatorname{Aut}_{E_{\breve{T}}}\right)(t):=\sum_{\mu \in \Lambda} \operatorname{Funct}\left(\operatorname{Eis}\left(\operatorname{Aut}_{E_{\breve{T}}}^{\mu}\right)\right) \cdot t^{\mu},
$$

viewed again as a function on $\operatorname{Bun}_{G}\left(\mathbb{F}_{q}\right)$ with values in $\widehat{\overline{\mathbb{Q}_{\ell}}[\Lambda]}$ (cf. Lemma 2.2.6).

Finally, for $\alpha \in \Delta^{+}$we introduce the (abelian) L-series $L\left(E_{\check{T}}, \alpha, t\right)$ to be the element of $\widehat{\overline{\mathbb{Q}_{\ell}}[\Lambda]}$ equal to $\sum_{n \in \mathbb{N}} \operatorname{Tr}\left(F r, H^{\cdot}\left(X^{(n)},\left(E_{\tilde{T}}^{\alpha}\right)^{(n)}\right) \otimes \overline{\mathbb{Q}_{\ell}}(n)\right) \cdot t^{n \cdot \alpha}$.

We have:

\section{Theorem 2.2.12.}

$$
\operatorname{Eis}_{m o d}\left(\operatorname{Aut}_{E_{\breve{T}}}\right)(t)=\operatorname{Eis}_{c l}\left(\operatorname{Aut}_{E_{\check{T}}}\right)(t) \cdot \prod_{\alpha \in \Delta^{+}} L\left(E_{\check{T}}, \alpha, t\right) .
$$

When $G=G L(n)$, the above result has been established by G. Laumon in [19].

Remark. It is well-known the the power series $\operatorname{Eis}_{c l}\left(\operatorname{Aut}_{E_{\breve{T}}}\right)(t)$ satisfies the functional equation of the form

$$
\operatorname{Eis}_{c l}\left(\operatorname{Aut}_{E_{\breve{T}}}\right)(t)=\operatorname{Eis}_{c l}\left(\operatorname{Aut}_{E_{\widetilde{T}}^{\mathrm{w}}}\right)(t) \cdot \frac{\prod_{\alpha \in \Delta^{+}} L\left(E_{\check{T}}^{\mathrm{w}}, \alpha, t\right)}{\prod_{\alpha \in \Delta^{+}} L\left(E_{\check{T}}, \alpha, t\right)},
$$

where $L\left(E_{\check{T}}, \alpha, t\right)$ are precisely the L-functions that appear in the formulation of Theorem 2.2.12. However, Theorem 2.2.4 implies that $\operatorname{Eis}_{\text {mod }}\left(\operatorname{Aut}_{E_{\breve{T}}}\right)(t)=\operatorname{Eis}_{\text {mod }}\left(\operatorname{Aut}_{E_{\tilde{T}}^{\mathrm{w}}}\right)(t)$ (if $E_{\breve{T}}$ is regular). Therefore, the fact that the ratio between Eis $_{\text {mod }}$ and Eis $_{c l}$ is the mentioned above product of L-functions is very natual from this point of view. 
It is quite remarkable that the Eisenstein series $\operatorname{Eis}_{m o d}\left(\operatorname{Aut}_{E_{\breve{T}}}\right)(t)$, which is more natural than $\operatorname{Eis}_{c l}\left(\mathrm{Aut}_{E_{\breve{T}}}\right)(t)$ from the geometric point of view (e.g. it comes from an object of $\operatorname{Sh}\left(\operatorname{Bun}_{G}\right)$, whose construction is self-dual in the Verdier sense) incorporates the L-function.

2.3. Non-principal Eisenstein series. We will now consider the case when $B$ is replaced by a parabolic $P$.

2.3.1. As in the previous subsection we define functor $\widetilde{\mathfrak{q}}_{P}^{! *}: \operatorname{Sh}\left(\operatorname{Bun}_{M}\right) \rightarrow \operatorname{Sh}(\widetilde{\operatorname{Bun}} P)$ by

$$
\mathcal{S} \rightarrow \mathrm{IC}_{\widetilde{\operatorname{Bun}}_{P}} \otimes \widetilde{\mathfrak{q}}_{P}^{*}(\mathcal{S}) \otimes\left(\overline{\mathbb{Q}_{\ell}}[1]\left(\frac{1}{2}\right)\right)^{\otimes-\operatorname{dim}\left(\operatorname{Bun}_{M}\right)} .
$$

Again, $\mathfrak{q}_{P}^{! *}$ will denote the functor

$$
\widetilde{j}_{P}^{*} \circ \widetilde{\mathfrak{q}}_{P}^{* *}: \operatorname{Sh}\left(\operatorname{Bun}_{M}\right) \rightarrow \operatorname{Sh}\left(\operatorname{Bun}_{P}\right) .
$$

Since the map $\mathfrak{q}_{P}: \operatorname{Bun}_{P} \rightarrow \operatorname{Bun}_{M}$ is smooth, $\mathfrak{q}_{P}^{! *}$ is essentially the ordinary pull-back.

The following theorem is parallel to Theorem 2.1.2:

Theorem 2.3.2. (a) The functor $\widetilde{\mathfrak{q}}_{P}^{* *}$ is exact and commutes with Verdier duality.

(b) If $\mathcal{S}$ is a perverse sheaf on $\operatorname{Bun}_{M}$, we have: $\widetilde{\mathfrak{q}}_{P}^{! *}(\mathcal{S}) \simeq \widetilde{j}_{P ! *} \circ \mathfrak{q}_{P}^{! *}(\mathcal{S})$.

We define the functor $\operatorname{Eis}_{M}^{G}: \operatorname{Sh}\left(\operatorname{Bun}_{M}\right) \rightarrow \operatorname{Sh}\left(\operatorname{Bun}_{G}\right)$ by

$$
\mathcal{S} \rightarrow \widetilde{\mathfrak{p}}_{P !} \circ \widetilde{\mathfrak{q}}_{P}^{! *}(\mathcal{S}) .
$$

As in Corollary 2.1.3, Theorem 2.3.2 insures that the functor $\operatorname{Eis}_{M}^{G}$ commutes with Verdier duality and maps pure complexes into pure ones.

2.3.3. Let $\mathcal{S}$ be a perverse sheaf on $\operatorname{Bun}_{M}$. One may attempt to give an alternative definition of the functor $\operatorname{Eis}_{M}^{G}$ as follows, using the compactification $\overline{\operatorname{Bun}}_{P}$ :

We can first pull back $\mathcal{S}$ onto $\operatorname{Bun}_{P}$ by means of $\mathcal{S} \rightarrow \mathfrak{q}_{P}^{! *}(\mathcal{S})$, then extend it by means of $j_{P \text { !* }}$ to obtain a perverse sheaf on $\overline{\operatorname{Bun}}_{P}$ and then take the direct image of the resulting sheaf with respect to the map $\overline{\mathfrak{p}}_{P}: \overline{\operatorname{Bun}}_{P} \rightarrow \mathrm{Bun}_{G}$. It will not be in general true that the two functors yield the same result. However, one can single out a class of perverse sheaves on $\operatorname{Bun}_{M}$ for which this will be true (cf. Sect. 6.4):

For a reductive group $G$ a perverse sheaf $\mathcal{S} \in \operatorname{Sh}\left(\operatorname{Bun}_{G}\right)$ is said to be "good" if for any sequence $\lambda_{1}, \ldots, \lambda_{n}$ of elements of $\Lambda_{G}^{+}$, the sheaf

$$
\left(H_{G}^{\lambda_{n}} \otimes \mathrm{id}^{n-1}\right) \circ \ldots \circ\left(H_{G}^{\lambda_{2}} \otimes \mathrm{id}\right) \circ H_{G}^{\lambda_{1}}(\mathcal{S})
$$

on $\operatorname{Bun}_{G} \times X^{n}$ is perverse as well.

For instance, when $G$ is abelian, every perverse sheaf on $\operatorname{Bun}_{G}$ is "good". For a general $G$, an automorphic sheaf that corresponds in the sense of Drinfeld-Langlands to a $\check{G}$-local system on $X$ is "good".

We have the following assertion, proved in Sect. 6.4:

Theorem 2.3.4. Let $\mathcal{S} \in \operatorname{Perv}\left(\operatorname{Bun}_{M}\right)$ be "good". Then there is a canonical isomorphism $\mathfrak{r}_{P !} \circ \widetilde{\mathfrak{q}}_{P}^{! *}(\mathcal{S}) \simeq j_{P ! *} \circ \mathfrak{q}_{P}^{! *}(\mathcal{S})$. 
Corollary 2.3.5. For a "good" perverse sheaf $\mathcal{S}$ on $\operatorname{Bun}_{M}$ the sheaf $\overline{\mathfrak{p}}_{P !} \circ j_{P ! *} \circ \mathfrak{q}_{P}^{! *}(\mathcal{S})$ on $\operatorname{Bun}_{G}$ is canonically isomorphic to $\operatorname{Eis}_{M}^{G}(\mathcal{S})$.

Remark. Let $G=G L(n)$. In this case there exists an open embedding of the stack Bun $_{G L(n)}$ into the stack $C_{0 h}$ that classifies coherent sheaves on $X$ of generic rank $n$. Following Laumon (cf. 19]) one can consider a functor

$$
\operatorname{Eis}_{n_{1}, \ldots, n_{k}}^{n}: \operatorname{Sh}\left(C o h_{n_{1}} \times \ldots \times C o h_{n_{k}}\right) \rightarrow \operatorname{Sh}\left(C o h_{n}\right),
$$

for $n=n_{1}+\ldots+n_{k}$.

Now let $\mathcal{S}$ be a "good" perverse sheaf on $\operatorname{Bun}_{G L\left(n_{1}\right)} \times \ldots \times \operatorname{Bun}_{G L\left(n_{k}\right)}$ and let $\mathcal{S}$ ' denote its Goresky-MacPherson extension on the whole of $\operatorname{Coh}_{n_{1}} \times \ldots \times C o h_{n_{k}}$. In this case we conjecture that the restriction of $\operatorname{Eis}_{n_{1}, \ldots, n_{k}}^{n}\left(\mathcal{S}^{\prime}\right)$ to $\operatorname{Bun}_{G L(n)} \subset C o h_{n}$ is canonically isomorphic to $\operatorname{Eis}_{M}^{G L(n)}(\mathcal{S})$, where $M=G L\left(n_{1}\right) \times \ldots \times G L\left(n_{k}\right)$.

2.3.6. In the case of non-principal Eisenstein series we have the following generalization of Theorem 2.1.5:

Theorem 2.3.7. For every $\lambda \in \Lambda_{G}^{+}$and $\mathcal{S} \in \operatorname{Sh}\left(\right.$ Bun $\left._{M}\right)$ there is a functorial isomorphism

$$
H_{G}^{\lambda} \circ \operatorname{Eis}_{M}^{G}(\mathcal{S}) \simeq \underset{\nu \in \Lambda_{M}^{+}}{\oplus}\left(\operatorname{Eis}_{M}^{G} \otimes \mathrm{id}\right) \circ H_{M}^{\nu}(\mathcal{S}) \otimes \operatorname{Hom}_{\check{M}}\left(U^{\nu}, V^{\lambda}\right)
$$

As in the case $P=B$ we can show in addition, that the isomorphism of functors in the above theorem is compatible with the convolution of Hecke functors.

Let $E_{\check{M}}$ be an $\check{M}$-local system on $X$ and let Aut $_{E_{\check{M}}}$ be an automorphic perverse sheaf on $\operatorname{Bun}_{M}$ that satisfies a Hecke eigen-property with respect to $E_{\check{M}}$. Let $E_{\breve{G}}$ denote a $\check{G}$-local system induced from $E_{\check{M}}$.

Corollary 2.3.8. The complex $\operatorname{Eis}_{M}^{G}\left(\operatorname{Aut}_{E_{\breve{M}}}\right)$ is a Hecke eigensheaf with respect to $E_{\breve{G}}$.

2.3.9. Finally, we have the following compatibility between the functors of Eisenstein series:

Theorem 2.3.10. For $\mathcal{S} \in \mathrm{Sh}\left(\mathrm{Bun}_{T}\right)$ there is a functorial isomorphism

$$
\operatorname{Eis}_{T}^{G} \simeq \operatorname{Eis}_{M}^{G} \circ \operatorname{Eis}_{T}^{M} .
$$

Moreover, this isomorphism of functors is compatible with the Hecke property in the sense that for every $\lambda \in \Lambda_{G}^{+}$the diagram

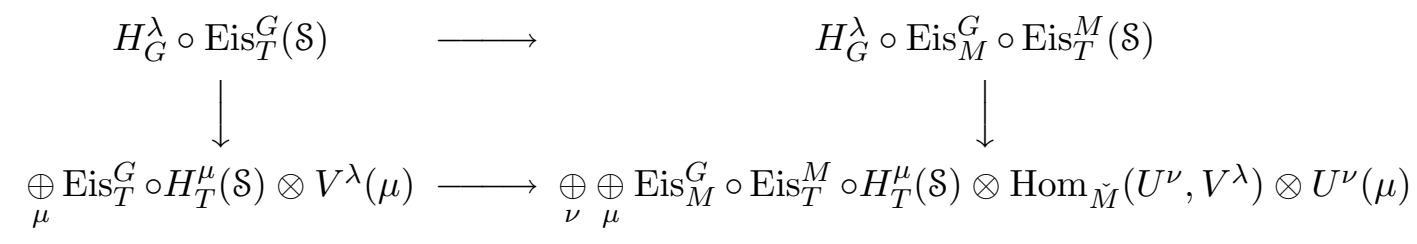

commutes.

Although the assertion of Theorem 2.3.10 seems very natural, the proof is quite non-trivial and it should be thought of as the main technical result of this paper. In particular, Theorem 2.1.8 will be a rather easy consequence of Theorem 2.3.10 via an explicit calculation in the case when $G$ has rank 1 . 


\section{Action of Hecke operators (the principal Case)}

3.1. The basic diagram. The construction described below will play an essential role in this paper.

3.1.1. Let $\lambda$ be a dominant coweight of $G$ and let us denote by $\bar{Z}$ the fiber product

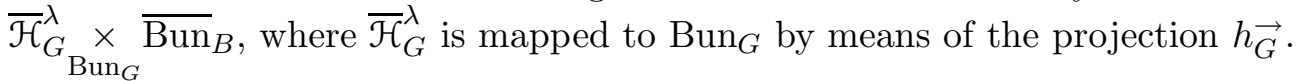

Proposition-Construction 3.1.2. There exists a map of stacks $\phi: \bar{Z} \rightarrow \overline{\operatorname{Bun}}_{B} \times X$ with $(\overline{\mathfrak{p}} \times \mathrm{id}) \circ \phi=\left(h_{G}^{\overleftarrow{G}} \times \pi\right) \circ{ }^{\prime} \overline{\mathfrak{p}}$

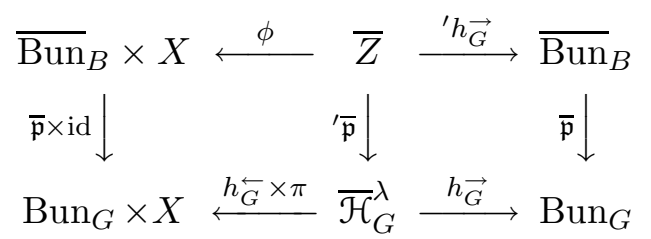

Note that the left square of this diagram is NOT Cartesian.

Proof. An $S$-point $z$ of $\bar{Z}$ consists by definition of the following data: $x \in \operatorname{Hom}(S, X)$; a pair of $G$-bundles $\mathcal{F}_{G}$ and $\mathcal{F}_{G}^{\prime}$ on $X \times S$ identified with one another outside on $X \times S-\Gamma_{x}$; a collection of line bundles $\mathcal{L}_{\mathcal{F}_{T}^{\prime}}^{\check{n}}$, each embedded as subsheaf into the corresponding $\mathcal{V}_{\mathcal{F}_{G}^{\prime}}^{\check{L}}$, such that the Plücker relations hold.

Recall that the fact that the pair $\left(\mathcal{F}_{G}, \mathcal{F}_{G}^{\prime}\right)$ belongs to $\overline{\mathcal{H}}_{G}^{\lambda}$ means, according to Sect. 2.1.4 that for each $\check{\lambda} \in \check{\Lambda}_{G}^{+}$we have:

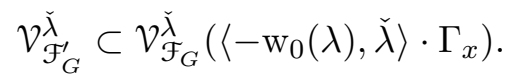

Therefore, the embedding $\kappa^{\prime \check{\lambda}}: \mathcal{L}_{\mathcal{F}_{T}^{\prime}}^{\check{L}} \hookrightarrow \mathcal{V}_{\mathcal{F}_{G}^{\prime}}^{\check{L}}$ gives rise to an embedding

$$
\kappa^{\check{\lambda}}: \mathcal{L}_{\mathcal{F}_{T}^{\prime}}^{\check{\lambda}}\left(\left\langle\mathrm{w}_{0}(\lambda), \check{\lambda}\right\rangle \cdot \Gamma_{x}\right) \hookrightarrow V_{\mathcal{F}_{G}}^{\check{\lambda}}
$$

and we set by definition $\phi(z)$ to be the object of $\overline{\mathrm{Bun}}_{B}$ that corresponds to $\mathcal{F}_{G}, \mathcal{F}_{T}:=\mathcal{F}_{T}^{\prime}\left(\mathrm{w}_{0}(\lambda) \cdot \Gamma_{x}\right)$ and the system of embeddings $\kappa^{\check{\lambda}}$ described above.

3.1.3. For every element $\nu \in \Lambda_{G}^{\text {pos }}$ consider the closed embedding

$$
i_{\nu}: \overline{\operatorname{Bun}}_{B} \times X \hookrightarrow \overline{\operatorname{Bun}}_{B} \times X,
$$

given by sending an object $\left(\mathcal{F}_{G}, \mathcal{F}_{T}, \kappa^{\check{\lambda}}: \mathcal{L}_{\mathcal{F}_{T}}^{\check{\lambda}} \hookrightarrow \mathcal{V}_{\mathcal{F}_{G}}^{\check{\lambda}}, x\right)$ to

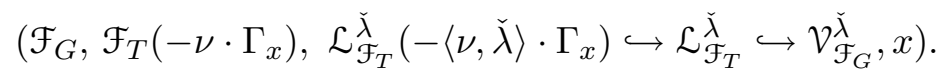

Theorem 3.1.4. $\phi_{!}\left(\mathrm{IC}_{\bar{Z}}\right) \simeq \underset{\nu \in \Lambda_{G}^{\mathrm{pos}}}{\oplus} i_{\nu !}\left(\mathrm{IC}_{\overline{\mathrm{Bun}}_{B} \times X}\right) \otimes V^{\lambda}\left(w_{0}(\lambda)+\nu\right)$.

Remark. An analogue of this theorem has been independently obtained by M. Finkelberg and I. Mirkovic. Our proof is slightly different from the argument of [11] and in Sect. 1 it will be generalized to the case when $B$ is replaced by a parabolic subgroup. 
3.1.5. Let us first show how Theorem 3.1.4 implies Theorem 2.1.5.

Proof. (of Theorem 2.1.5)

For $\nu \in \Lambda$ let us denote by $m^{\nu}$ the map

$$
\operatorname{Bun}_{T} \times X \simeq \overline{\mathcal{H}}_{T}^{\nu} \stackrel{h_{T}}{\longrightarrow} \operatorname{Bun}_{T} .
$$

By definition, for $\mathcal{S} \in \operatorname{Sh}\left(\operatorname{Bun}_{T}\right)$, the sheaf $m^{\nu *}(\mathcal{S})$ is canonically the same as $H_{T}^{\nu}(\mathcal{S})$.

Lemma 3.1.6. We have:

(a) The maps $\overline{\mathfrak{q}} \circ h_{G}$ and $m^{\mathrm{w}_{0}(\lambda)} \circ(\overline{\mathfrak{q}} \times \mathrm{id}) \circ \phi$ from $\bar{Z}$ to $\operatorname{Bun}_{T}$ coincide.

(b) For every $\nu$, the maps $(\overline{\mathfrak{q}} \times \mathrm{id}) \times i_{\nu}$ and $\left(m^{\nu} \times \mathrm{id}\right) \circ(\overline{\mathfrak{q}} \times \mathrm{id})$ from $\overline{\operatorname{Bun}}_{B} \times X$ to $\operatorname{Bun}_{T} \times X$ coincide.

For the proof of Theorem 2.1.5, observe that for an object $\mathcal{S} \in \operatorname{Sh}\left(\operatorname{Bun}_{T}\right)$, the sheaf $h_{G}{ }^{*} \circ \operatorname{Eis}(\mathcal{S})$ on $\bar{Z}$ identifies (by base change and the projection formula) with

$$
' \overline{\mathfrak{p}}_{!}\left({ }^{\prime} h_{G} \overrightarrow{\mathrm{C}}^{*} \circ \overline{\mathfrak{q}}^{*}(\mathcal{S}) \otimes{ }^{\prime} h_{G} \overrightarrow{\mathrm{C}}^{*}\left(\mathrm{IC}_{\overline{\mathrm{Bun}}_{B}}\right) \otimes \overline{\mathfrak{p}}^{*}\left(\mathrm{IC}_{\overline{\mathcal{H}}_{G}^{\lambda}}\right)\right) \otimes\left(\overline{\mathbb{Q}_{\ell}}\left(\frac{1}{2}\right)[1]\right)^{\otimes-\operatorname{dim}\left(\operatorname{Bun}_{T}\right)} .
$$

However, the map $h_{G}: \overline{\mathcal{H}}_{G}^{\lambda} \rightarrow \operatorname{Bun}_{G}$ decomposes locally in the smooth topology with respect to $\operatorname{Bun}_{G}$ into a direct product, which implies that

$$
\mathrm{IC}_{\bar{Z}} \simeq \overline{\mathfrak{p}}^{*}\left(\mathrm{IC}_{\overline{\mathcal{H}}_{G}^{\lambda}}\right) \otimes h_{G} \vec{G}^{*}\left(\mathrm{IC}_{\overline{\operatorname{Bun}}_{B}}\right) \otimes\left(\overline{\mathbb{Q}_{\ell}}\left(\frac{1}{2}\right)[1]\right)^{\otimes-\operatorname{dim}\left(\operatorname{Bun}_{G}\right)}
$$

Therefore,

$$
h_{G}^{*} \circ \operatorname{Eis}(\mathcal{S}) \simeq \overline{\mathfrak{p}}_{!}\left({ }^{\prime} h_{G} \vec{G}^{*} \circ \overline{\mathfrak{q}}^{*}(\mathcal{S}) \otimes \mathrm{IC}_{Z}\right) \otimes\left(\overline{\mathbb{Q}_{\ell}}\left(\frac{1}{2}\right)[1]\right)^{\otimes-\operatorname{dim}\left(\operatorname{Bun}_{T}\right)+\operatorname{dim}\left(\operatorname{Bun}_{G}\right)}
$$

and hence

$$
H_{G}^{\lambda} \circ \operatorname{Eis}(\mathcal{S}) \simeq(\overline{\mathfrak{p}} \times \mathrm{id}) ! \circ \phi_{!}\left({ }^{\prime} h_{G} \vec{G}^{*} \circ \overline{\mathfrak{q}}^{*}(\mathcal{S}) \otimes \mathrm{IC}_{Z}\right)\left(\overline{\mathbb{Q}_{\ell}}\left(\frac{1}{2}\right)[1]\right)^{\otimes-\operatorname{dim}\left(\operatorname{Bun}_{T}\right)} .
$$

Acording to point (a) of Lemma 3.1.6 and the projection formula, the last expression can be rewritten as

$$
(\overline{\mathfrak{p}} \times \mathrm{id}) !\left(\phi_{!}\left(\mathrm{IC}_{\bar{Z}}\right) \otimes(\overline{\mathfrak{q}} \times \mathrm{id})^{*} \circ m^{\mathrm{w}_{0}(\lambda) *}(\mathcal{S})\right) \otimes\left(\overline{\mathbb{Q}_{\ell}}\left(\frac{1}{2}\right)[1]\right)^{\otimes-\operatorname{dim}\left(\operatorname{Bun}_{T}\right)}
$$

and finally, by applying Theorem 3.1.4 and the projection formula we obtain that the sheaf $H_{G}^{\lambda} \circ \operatorname{Eis}(\mathcal{S})$ identifies with the direct sum over $\nu \in \Lambda_{G}^{\text {pos }}$ of the expressions

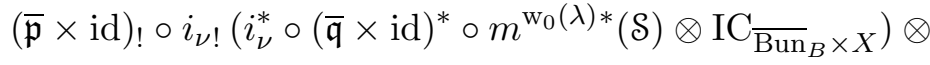

$$
\begin{aligned}
& \otimes V^{\lambda}\left(\mathrm{w}_{0}(\lambda)+\nu\right) \otimes\left(\overline{\mathbb{Q}_{\ell}}\left(\frac{1}{2}\right)[1]\right)^{\otimes-\operatorname{dim}\left(\operatorname{Bun}_{T}\right)} .
\end{aligned}
$$

Now, by Lemma 3.1.6(b),

$$
i_{\nu}^{*} \circ(\overline{\mathfrak{q}} \times \mathrm{id})^{*} \circ m^{\mathrm{w}_{0}(\lambda) *}(\mathcal{S}) \simeq(\overline{\mathfrak{q}} \times \mathrm{id})^{*} \circ H_{T}^{\mathrm{w}_{0}(\lambda)+\nu}(\mathcal{S}) .
$$

Since the maps $(\overline{\mathfrak{p}} \times \mathrm{id}) \circ i_{\nu}$ and $\overline{\mathfrak{p}} \times$ id from $\overline{\operatorname{Bun}}_{B} \times X \rightarrow \operatorname{Bun}_{G} \times X$ coincide, we obtain that $H_{G}^{\lambda} \circ \operatorname{Eis}(\mathcal{S})$ can be identified with 


$$
\begin{aligned}
& \underset{\nu \in \Lambda_{G}^{\mathrm{pos}}}{\oplus}(\overline{\mathfrak{p}} \times \mathrm{id})_{!}\left((\overline{\mathfrak{q}} \times \mathrm{id})^{*} \circ H_{T}^{\mathrm{w}_{0}(\lambda)+\nu}(\mathcal{S}) \otimes \mathrm{IC}_{\overline{\operatorname{Bun}_{B}} \times X}\right) \otimes V^{\lambda}\left(\mathrm{w}_{0}(\lambda)+\nu\right) \otimes \\
& \otimes\left(\overline{\mathbb{Q}_{\ell}}\left(\frac{1}{2}\right)[1]\right)^{\otimes-\operatorname{dim}\left(\mathrm{Bun}_{T}\right)} \simeq \underset{\nu \in \Lambda_{G}^{\mathrm{pos}}}{\oplus}(\text { Eis } \otimes \mathrm{id}) \circ H_{T}^{\mathrm{w}_{0}(\lambda)+\nu}(\mathcal{S}) \otimes V^{\lambda}\left(\mathrm{w}_{0}(\lambda)+\nu\right),
\end{aligned}
$$

as required.

The fact that the isomorphism $H_{G}^{\lambda} \circ \operatorname{Eis}(\mathcal{S}) \simeq \underset{\nu \in \Lambda}{\oplus}(\operatorname{Eis} \otimes \mathrm{id}) \circ H_{T}^{\nu}(\mathcal{S}) \otimes V^{\lambda}(\nu)$ constructed above is compatible with the convolution of Hecke functors will be explained in the next section in a more general context.

Thus, modulo Theorem 3.1.4, we have established Theorem 2.1.5 together with Theorem 2.2.2.

3.2. Preliminaries on the affine Grassmannian. In this subsection we will review several facts concerning the affine Grassmannian corresponding to the group $G$. These facts will be needed for the proof of Theorem 3.1.4 as well as for the rest of the paper; the proofs can be found in [20] or in [3].

3.2.1. Let $x \in X$ be a point and let $\mathcal{D}_{x}$ (resp., $\mathcal{D}_{x}^{*}$ ) be the formal disc (resp., the formal punctured disc) around $x$. Let $G\left(\mathcal{O}_{x}\right)$ (resp., $G\left(\mathcal{K}_{x}\right)$ ) be the group-scheme (resp., group-indscheme) that classifies maps from $\mathcal{D}_{x}$ (resp., from $\mathcal{D}_{x}^{*}$ ) to $G$.

The affine Grassmannian $\operatorname{Gr}_{G}$ is, by definition, the quotient $G\left(\mathcal{K}_{x}\right) / G\left(\mathcal{K}_{x}\right)$. (Sometimes, in order to emphasize the dependence on $x$ we will put a subscript $\operatorname{Gr}_{G x}$.) In other words, $\operatorname{Gr}_{G}$ is an indscheme that classifies the data of $\left(\mathcal{F}_{G}, \beta\right)$, where $\mathcal{F}_{G}$ is a $G$-bundle on $\mathcal{D}_{x}$ and $\beta$ is its trivialization: $\mathcal{F}_{G} \simeq \mathcal{F}_{G}^{0}$ on $\mathcal{D}_{x}^{*}$. (We leave it to the reader to formulate what this means on the level of $S$-points, cf. [15]).

Being an indscheme, $\mathrm{Gr}_{G}$ is a union of its closed finite-dimensional subschemes. Therefore, the notion of a perverse sheaf on $\mathrm{Gr}_{G}$ makes sense. (By definition, every such perverse is supported on a finite-dimensional subscheme of $\mathrm{Gr}_{G}$.)

For $\lambda \in \Lambda_{G}^{+}$let $\overline{\mathrm{Gr}}_{G}^{\lambda}$ denote the closed sub-scheme of $\mathrm{Gr}_{G}$ that corresponds to pairs $\left(\mathcal{F}_{G}, \beta\right)$ as above for which $\mathcal{V}_{\mathcal{F}_{G}^{0}}(-\langle\lambda, \check{\lambda}\rangle \cdot x) \subset \mathcal{V}_{\mathcal{F}_{G}}^{\check{\lambda}}$ for every $G$-module $\mathcal{V}$ such all its weights are $\leq \check{\lambda}$. (Here $x$ denotes, of course, the closed point of $\mathcal{D}_{x}$.)

By construction, each $\overline{\mathrm{Gr}}_{G}^{\lambda}$ is a finite-dimensional projective variety, stable under the left $G\left(\mathcal{O}_{x}\right)$-action. We have:

$$
\overline{\mathrm{Gr}}_{G}^{\lambda^{\prime}} \subset \overline{\mathrm{Gr}}_{G}^{\lambda} \text { if and only if } \lambda^{\prime} \leq \lambda \text {. }
$$

In addition, it is known (cf. [20]) that $\overline{\mathrm{Gr}}_{G}^{\lambda}$ contains a unique dense $G\left(\mathcal{O}_{x}\right)$-orbit, denoted $\mathrm{Gr}_{G}^{\lambda}$. If $t_{x}$ is a uniformizer at $x$, then $\operatorname{Gr}_{G}^{\lambda}=G\left(\mathcal{O}_{x}\right) \cdot t_{x}^{\lambda}$, where $t_{x}^{\lambda}$ is the corresponding point of $T\left(\mathcal{K}_{x}\right) \subset G\left(\mathcal{K}_{x}\right)$.

Thus, we obtain a stratification of $\mathrm{Gr}_{G}$ by the sub-schemes $\operatorname{Gr}_{G}^{\lambda}$. ?

\footnotetext{
${ }^{3}$ In this paper, the term "stratification" will be used in the following weak sense:

Let $Y$ be a scheme (resp., indscheme, stack, indstack) and let $Y_{i}$ be a collection of locally closed subschemes of $Y$. We say that they form a stratification if $Y\left(\overline{\mathbb{F}_{q}}\right)$ is a disjoint union $\cup Y_{i}\left(\overline{\mathbb{F}_{q}}\right)$.
} 
For $\lambda \in \Lambda_{G}^{+}$let $\mathcal{A}_{G}^{\lambda}$ denote the intersection cohomology sheaf $\mathrm{IC}_{\overline{\mathrm{Gr}}_{G}^{\lambda}}$. Let $\mathrm{Sph}_{G}$ denote the full abelian subcategory of $\operatorname{Perv}\left(\mathrm{Gr}_{G}\right)$ formed by direct sums of the perverse sheaves $\mathcal{A}_{G}^{\lambda}$. By definition, every object of $\mathrm{Sph}_{G}$ is $G\left(\mathcal{O}_{x}\right)$-equivariant.

Note that $G\left(\mathcal{O}_{x}\right)$-equivariant perverse sheaves on $\mathrm{Gr}_{G}$ can be thought of as "perverse sheaves" on the stack that classifies triples $\left(\mathcal{F}_{G}^{1}, \mathcal{F}_{G}^{2}, \beta\right)$, where $\mathcal{F}_{G}^{i}$ are $G$-bundles on $X$ $\mathcal{D}_{x}$ and $\beta$ is an isomorphism $\left.\left.\mathcal{F}_{G}^{1}\right|_{\mathcal{D}_{x}^{*}} \rightarrow \mathcal{F}_{G}^{2}\right|_{\mathcal{D}_{x}^{*}}$. The latter stack carries a natural involution given by the flip $\mathcal{F}_{G}^{1} \leftrightarrow \mathcal{F}_{G}^{2}$. This involution gives rise to a covariant functor, denoted $*$, from the category of $G\left(\mathcal{O}_{x}\right)$-equivariant perverse sheaves $\mathrm{Gr}_{G}$ to itself. We have $* \mathcal{A}_{G}^{\lambda} \simeq \mathcal{A}_{G}^{-\mathrm{w}_{0}(\lambda)}$, hence, * preserves the subcategory $\operatorname{Sph}_{G}$.

3.2.2. Now we will recall the definition of the convolution operation on the category

$\operatorname{Sph}_{G}$. Consider the indscheme $\operatorname{Conv}_{G}:=\operatorname{Gr}_{G} \stackrel{G\left(\mathcal{O}_{x}\right)}{\times} G\left(\mathcal{K}_{x}\right)$. By definition, it classifies the data of $\left(\mathcal{F}_{G}, \mathcal{F}_{G}^{\prime}, \widetilde{\beta}, \beta^{\prime}\right)$, where $\mathcal{F}_{G}$ and $\mathcal{F}_{G}^{\prime}$ are a $G$-bundles on $\mathcal{D}_{x}, \widetilde{\beta}$ is an iso-

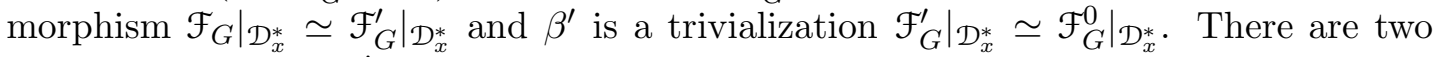
projections $p r$ and $p r^{\prime}$ from $\operatorname{Conv}_{G}$ to $\operatorname{Gr}_{G}$ :

For $g_{1} \times g_{2} \in G\left(\mathcal{K}_{x}\right) \times \mathrm{Gr}_{G}$,

$$
\operatorname{pr}\left(g_{1} \times g_{2}\right)=g_{1} \cdot g_{2} \bmod G\left(\mathcal{O}_{x}\right) \text { and } p r^{\prime}\left(g_{1} \times g_{2}\right)=g_{2} \bmod G\left(\mathcal{O}_{x}\right) .
$$

In the functorial language, $p r^{\prime}$ and $p r$ act as follows:

$$
\operatorname{pr}^{\prime}\left(\mathcal{F}_{G}, \mathcal{F}_{G}^{\prime}, \widetilde{\beta}, \beta^{\prime}\right)=\left(\mathcal{F}_{G}^{\prime}, \beta^{\prime}\right) \text { and } \operatorname{pr}\left(\mathcal{F}_{G}, \mathcal{F}_{G}^{\prime}, \widetilde{\beta}, \beta^{\prime}\right)=\left(\mathcal{F}_{G}, \widetilde{\beta} \circ \beta^{\prime}\right) \text {. }
$$

If $\mathcal{S}^{1}$ is a $G\left(\mathcal{O}_{x}\right)$-equivariant perverse sheaf on $\mathrm{Gr}_{G}$ and $\mathcal{S}_{2}$ is an arbitrary perverse sheaf on $\mathrm{Gr}_{G}$, we can form their twisted external product $\mathcal{S}^{1} \widetilde{\nabla} \mathcal{S}^{2}$ (cf. Sect. 0.4), which is a perverse sheaf on $\operatorname{Conv}_{G}$. The convolution $\mathcal{S}^{1} \star \mathcal{S}^{2} \in \operatorname{Sh}\left(\operatorname{Gr}_{G}\right)$ is defined as $p r_{!}\left(\mathcal{S}^{1} \widetilde{\nabla} \mathcal{S}^{2}\right)$. We have the following result:

Proposition 3.2.3. In the above situation $\mathcal{S}^{1} \star \mathcal{S}^{2}$ is a perverse sheaf.

Remark. Proposition 3.2.3 has been first established in [20] under the assumption that $\mathcal{S}_{2}$ is also $G\left(\mathcal{O}_{x}\right)$-equivariant. The proof in the general case is given in 15 .

Assume now that both $\mathcal{S}_{1}$ and $\mathcal{S}_{2}$ belong to $\operatorname{Sph}_{G}$. The Proposition 3.2.3 and the decomposition theorem (using the fact the each $\operatorname{Gr}_{G}^{\lambda}$ is simply-connected) imply that $\delta^{1} \star \delta^{2}$ is also an object of $\mathrm{Sph}_{G}$. In addition, it follows from the definitions that there is a canonical isomorphism $*\left(\mathcal{S}_{1} \star \mathcal{S}_{2}\right) \simeq\left(* \mathcal{S}_{2}\right) \star\left(* \mathcal{S}_{1}\right)$.

3.2.4. We will now reformulate the definition of $\mathrm{Gr}_{G}$ using the global curve $X$. We claim that $\operatorname{Gr}_{G}$ is the indscheme that classifies the data of $\left(\mathcal{F}_{G}, \beta\right)$, where $\mathcal{F}_{G}$ is a $G$-bundle on the curve $X$ and $\beta$ is its trivialization on $X-x$.

Indeed, to $\left(\mathcal{F}_{G}, \beta\right)$ as above, we can attach $\left.\mathcal{F}_{G}\right|_{\mathcal{D}_{x}}$, which is a $G$-bundle on $\mathcal{D}_{x}$ and $\beta$

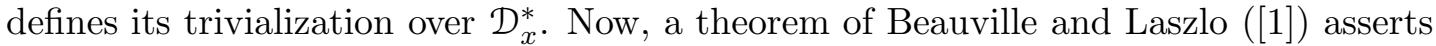
that the above map from the set of pairs $\left(F_{G}, \beta\right)$ "on $X$ " to that "on $\mathcal{D}_{x}$ " is a bijection.

Consider the stack $\operatorname{Bun}_{G}$. Let ${ }_{x} \mathcal{G}$ denote the canonical $G\left(\mathcal{O}_{x}\right)$-torsor over $\operatorname{Bun}_{G}$, whose fiber over $\mathcal{F}_{G} \in \operatorname{Bun}_{G}$ is the set of isomorphisms $\left.\left.\mathcal{F}_{G}\right|_{\mathcal{D}_{x}} \simeq \mathcal{F}_{G}^{0}\right|_{\mathcal{D}_{x}}$.

Recall the stack $\mathcal{H}_{G}$ and let ${ }_{x} \mathcal{H}_{G}$ denote its fiber over $\operatorname{Bun}_{G} \times x \subset \operatorname{Bun}_{G} \times X$; let ${ }_{x} \overline{\mathcal{H}}_{G}^{\lambda} \subset{ }_{x} \mathcal{H}_{G}$ be the corresponding finite-dimensional substack. 
The above mentioned result of [1] implies that both projections $h_{G}^{\leftarrow}$ and $h_{G}$ realize ${ }_{x} \mathcal{H}_{G}$ as a fibration over $\operatorname{Bun}_{G}$ with the typical fiber $\mathrm{Gr}_{G}$ :

$$
{ }_{x} \mathcal{H}_{G} \simeq \operatorname{Gr}_{G} \stackrel{G\left(\mathcal{O}_{x}\right)}{\times}{ }_{x} \mathcal{G} .
$$

We call these identifications id ${ }^{l}$ and $\mathrm{id}^{r}$, respectively. By definition, under $\mathrm{id}^{l},{ }_{x} \overline{\mathcal{H}}_{G}^{\lambda}$ goes over to the substack $\overline{\operatorname{Gr}}_{G}^{\lambda} \stackrel{G\left(\mathcal{O}_{x}\right)}{\times}{ }_{x} \mathcal{G} \subset \operatorname{Gr}_{G} \stackrel{G\left(\mathcal{O}_{x}\right)}{\times}{ }_{x} \mathcal{G}$.

We define the locally closed substack ${ }_{x} \mathcal{H}_{G}^{\lambda}$ of $\overline{\mathcal{H}}_{G}^{\lambda}$ as $\operatorname{Gr}_{G}^{\lambda} \stackrel{G\left(\mathcal{O}_{x}\right)}{\times}{ }_{x} \mathcal{G}$. In what follows, for $\left(\mathcal{F}_{G}, \mathcal{F}_{G}^{\prime}, \beta\right) \in{ }_{x} \mathcal{H}_{G}^{\lambda}$, we will say that " $\mathcal{F}_{G}^{\prime}$ is in position $\lambda$ with respect to $\mathcal{F}_{G}$ ".

Thus, to $\mathcal{S} \in \operatorname{Sph}_{G}$ and an object $\mathcal{T} \in \operatorname{Sh}\left(\operatorname{Bun}_{G}\right)$ we can attach their twisted external products $(\mathcal{S} \widetilde{\otimes} \mathcal{T})^{l}$ and $(\mathcal{S} \widetilde{\otimes} \mathcal{T})^{r}$. In particular, the intersection cohomology sheaf $\mathrm{IC}_{x} \overline{\mathcal{H}}_{G}^{\lambda}$ is nothing but $\left(\mathcal{A}_{G}^{\lambda} \widetilde{\otimes} \mathrm{IC}_{\mathrm{Bun}_{G}}\right)^{l}$.

We introduce the Hecke functors ${ }_{x} H_{G}^{\leftarrow}(\cdot, \cdot)$ and ${ }_{x} H_{G}(\cdot, \cdot)$ from $\operatorname{Sph}_{G} \times \operatorname{Sh}\left(\operatorname{Bun}_{G}\right)$ to $\operatorname{Sh}\left(\operatorname{Bun}_{G}\right)$ as follows:

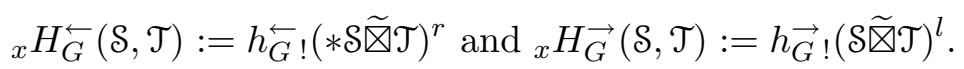

Since every $\overline{\mathrm{Gr}}_{G}^{\lambda}$ is complete, the projections $h_{G}^{\leftarrow}$ and $h_{G}$ are proper and the Hecke functors commute with Verdier duality. Moreover, they are compatible with the tensor structure on $\mathrm{Sph}_{G}$ in the sense that there exist functorial isomorphisms:

${ }_{x} H_{G}^{\leftarrow}\left(\mathcal{S}_{1},{ }_{x} H_{G}^{\leftarrow}\left(\mathcal{S}_{2}, \mathcal{T}\right)\right) \simeq{ }_{x} H_{G}^{\leftarrow}\left(\mathcal{S}_{1} \star \mathcal{S}_{2}, \mathcal{T}\right)$ and ${ }_{x} H_{G}\left(\mathcal{S}_{1},{ }_{x} H_{G}\left(\mathcal{S}_{2}, \mathcal{T}\right)\right) \simeq{ }_{x} H_{G}\left(\mathcal{S}_{2} \star \mathcal{S}_{1}, \mathcal{T}\right)$.

Finally, let us observe that there is a canonical isomorphism ${ }_{x} H_{G}^{\leftarrow}(\mathcal{S}, \mathcal{T}) \simeq{ }_{x} H_{G}(* \mathcal{S}, \mathcal{T})$ and for a fixed $\mathcal{S} \in \mathrm{Sph}_{G}$, the functors

$$
\mathcal{T} \rightarrow{ }_{x} H_{G}^{\leftarrow}(\mathcal{S}, \mathcal{T}) \text { and } \mathcal{T} \rightarrow{ }_{x} H_{G}(\mathbb{D}(\mathcal{S}), \mathcal{T})
$$

are mutually (both left and right) adjoint.

3.2.5. In addition to the stratification by the $\operatorname{Gr}_{G}^{\lambda}$ 's, the indscheme $\mathrm{Gr}_{G}$ is stratified by the so-called semiinfinite orbits:

Consider the standard Borel structure on $\mathcal{F}_{G}^{0}$ given by the collection of the embeddings

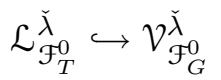

and for $\nu \in \Lambda$ let $\bar{S}_{G}^{\nu}$ denote the closed ind-subscheme of $\mathrm{Gr}_{G}$ corresponding to those pairs $\left(\mathcal{F}_{G}, \beta\right)$, for which the meromorphic map

$$
\mathcal{L}_{\mathcal{F}_{T}^{0}}^{\check{\lambda}}(-\langle\nu, \check{\lambda}\rangle \cdot x) \rightarrow \mathcal{V}_{\mathcal{F}_{G}^{0}}^{\check{\lambda}} \stackrel{\beta}{\longrightarrow} \mathcal{V}_{\mathcal{F}_{G}}^{\check{\lambda}}
$$

does not have a pole $\forall \check{\lambda} \in \check{\Lambda}_{G}^{+}$.

Inside $\bar{S}_{G}^{\nu}$ there is an open ind-subscheme $S_{G}^{\nu}$ corresponding to those pairs $\left(\mathcal{F}_{G}, \beta\right)$, for which the above map

$$
\mathcal{L}_{\mathcal{F}_{T}^{0}}^{\check{\lambda}}(-\langle\nu, \check{\lambda}\rangle \cdot x) \rightarrow \mathcal{V}_{\mathcal{F}_{G}}^{\check{\lambda}}
$$

does not have a zero either. Hence, $\operatorname{Gr}_{G}$ (resp., $\bar{S}_{G}^{\nu^{\prime}}$ ) is stratified by the $S_{G}^{\nu}, \nu \in \Lambda_{G}$ (resp., by the $S_{G}^{\nu}, \nu \leq \nu^{\prime}$ ). 
Consider the group-indschemes $U\left(\mathcal{K}_{x}\right) \subset U\left(\mathcal{K}_{x}\right) T\left(\mathcal{O}_{x}\right) \subset G\left(\mathcal{K}_{x}\right)$. For each $\nu \in \Lambda_{G}$, $S_{G}^{\nu}$ is stable with respect to the $U\left(\mathcal{K}_{x}\right) T\left(\mathcal{O}_{x}\right)$-action on $\operatorname{Gr}_{G}$. (Moreover, it is easy to see that $S_{G}^{\nu}$ is isomorphic to the quotient of $U\left(\mathcal{K}_{x}\right)$ by a certain group-subscheme.)

The following assertion (due to Mirkovic and Vilonen, [21]) will play a central role in this paper:

Proposition 3.2.6. Consider the intersection $\mathrm{Gr}_{G}^{\lambda} \cap S_{G}^{\nu}$.

(a) It is empty unless $\mathrm{w}_{0}(\lambda) \leq \nu \leq \lambda$. For $\nu=\lambda$, it is open inside $\operatorname{Gr}_{G}^{\lambda}$ (and hence is smooth and irreducible). For $\nu=\mathrm{w}_{0}(\lambda)$, it is isomorphic to the point-scheme.

(b) $\operatorname{dim}\left(\operatorname{Gr}_{G}^{\lambda} \cap S_{G}^{\nu}\right) \leq\langle\lambda+\nu, \check{\rho}\rangle$.

3.2.7. It has been known since the works of Lusztig [20], Ginzburg [16] and MirkovicVilonen [21] that the functor $\star: \mathrm{Sph}_{G} \times \mathrm{Sph}_{G} \mapsto \mathrm{Sph}_{G}$ prolongs to a structure of tensor category on $\mathrm{Sph}_{G}$, and as such it is equivalent to the category of finite-dimensional representations of the Langlands-dual group $\check{G}$. We will need this statement in the following formulation $([3])$ :

Theorem 3.2.8. (a) The convolution product $\mathcal{S}^{1}, \mathcal{S}^{2} \rightarrow \mathcal{S}^{1} \star \mathcal{S}^{2}$ admits associativity and commutativity constraints, i.e. $\mathrm{Sph}_{G}$ has a structure of a (symmetric) tensor category. Moreover, the functor

$$
\operatorname{gRes}_{T}^{G}: \operatorname{Sph}_{G} \rightarrow \Lambda \text {-graded vector spaces }
$$

given by

$$
\operatorname{gRes}_{T}^{G}(\mathcal{S})=\bigoplus_{\nu \in \Lambda} H_{c}^{\langle\nu, 2 \check{\rho}\rangle}\left(S_{G}^{\nu},\left.\mathcal{S}\right|_{S_{G}^{\nu}}\right) \otimes \overline{\mathbb{Q}_{\ell}}(\langle\nu, \check{\rho}\rangle)
$$

has a natural structure of a tensor functor.

(b) The algebriac group corresponding (by Tannaka's theorem) to $\mathrm{Sph}_{G}$ and the composition

$$
\text { Forget } \circ \operatorname{gRes}_{T}^{G}: \operatorname{Sph}_{G} \rightarrow \Lambda \text {-graded vector spaces } \rightarrow \text { vector spaces }
$$

is canonically isomorphic to the Langlands dual group $\check{G}$, in such a way that the equivalence

$$
F_{G}: \operatorname{Sph}_{G} \rightarrow \operatorname{Rep}(\check{G})
$$

has the following properties:

- $\operatorname{gRes}_{T}^{G}$ goes over to the natural restriction functor $\operatorname{Res}_{T}^{G}: \operatorname{Rep}(\check{G}) \rightarrow \operatorname{Rep}(\check{T})$, i.e.

$$
\operatorname{Res}_{T}^{G} \circ F_{G} \simeq \operatorname{gRes}_{T}^{G} .
$$

- $F_{G}\left(\mathcal{A}_{G}^{\lambda}\right)=V^{\lambda} \in \operatorname{Rep}(\check{G})$.

- The countravariant self-functor $\mathcal{S} \rightarrow \mathbb{D}(* \mathcal{S})$ on $\mathrm{Sph}_{G}$ goes over to the dualization functor on $\operatorname{Rep}(\check{G})$.

The following assertion can be obtained by combining Theorem 3.2 .8 and Proposition 3.2.6: 
Corollary 3.2.9. For $\lambda \in \Lambda_{G}^{+}$and $\nu \in \Lambda$ consider the $\overline{\mathbb{Q}_{\ell}}$-vector space $H_{c}^{i}\left(S_{G}^{\nu},\left.\mathcal{A}_{G}^{\lambda}\right|_{S_{G}^{\nu}} ^{\nu}\right) \otimes \overline{\mathbb{Q}_{\ell}}(\langle\nu, \check{\rho}\rangle)$. We have:

(a) It is zero for $i>\langle\nu, 2 \check{\rho}\rangle$.

(b) For $i=\langle\nu, 2 \check{\rho}\rangle$ it identifies with $V^{\lambda}(\nu)$. Moreover,

$$
V^{\lambda}(\nu) \simeq H_{c}^{\langle\lambda+\nu, 2 \breve{\rho}\rangle}\left(\operatorname{Gr}_{G}^{\lambda} \cap S_{G}^{\nu}, \overline{\mathbb{Q}_{\ell}}(\langle\lambda+\nu, \check{\rho}\rangle)\right) .
$$

In particular, we infer that $V^{\lambda}(\nu)$ has a basis given by the irreducible components of the scheme $\operatorname{Gr}_{G}^{\lambda} \cap S_{G}^{\nu}$ which have (the maximal possible) dimension $\langle\lambda+\nu, \check{\rho}\rangle$.

Remark. The paper 21] contains an even stronger assertion, namely that the cohomology group $H^{i}\left(S_{G}^{\nu},\left.\mathcal{A}_{G}^{\lambda}\right|_{S_{G}^{\nu}}\right)$ vanishes unless $i=\langle\nu, 2 \check{\rho}\rangle$ and that the scheme $\operatorname{Gr}_{G}^{\lambda} \cap S_{G}^{\nu}$ is of pure dimension $\langle\nu, 2 \check{\rho}\rangle$. However, for our purposes the formulation of Corollary 3.2 .9 will be sufficient.

\subsection{Proof of Theorem 3.1.4.}

3.3.1. For a point $x \in X$, let ${ }_{x} \bar{Z}$ be the pre-image ${ }^{\prime} \overline{\mathfrak{p}}^{-1}\left({ }_{x} \overline{\mathcal{H}}_{G}^{\lambda}\right) \subset \bar{Z}$. (By abuse of notation we will continue to denote by $\phi$ (resp., ' $h_{G}, h_{G}, h_{G}^{\leftarrow}, i_{\nu}$ ) the corresponding maps when $\overline{\operatorname{Bun}}_{B} \times X$ is replaced by $\overline{\operatorname{Bun}}_{B}=\overline{\operatorname{Bun}}_{B} \times x$.)

We have the following diagram:

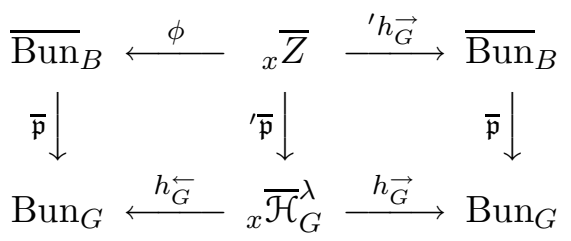

To simplify the notation, we will prove the following version of Theorem 3.1.4:

Theorem 3.3.2. $\phi_{!}\left(\mathrm{IC}_{x} \bar{Z}\right) \simeq \underset{\nu \in \Lambda_{G}^{\mathrm{pos}}}{\oplus} i_{\nu !}\left(\mathrm{IC}_{\overline{\mathrm{Bun}}_{B}}\right) \otimes V^{\lambda}\left(\mathrm{w}_{0}(\lambda)+\nu\right)$.

The proof of Theorem 3.1.4 is no diffrent from the presented below proof of Theorem 3.3.2.

For $\nu \in \Lambda_{G}^{\text {pos }}$, let ${ }_{x, \nu} \overline{\operatorname{Bun}}_{B}$ (resp., $x_{x, \geq \nu} \overline{\operatorname{Bun}}_{B}$ ) be the closed (resp., locally closed) substack of $\overline{\mathrm{Bun}}_{B}$ that corresponds to those triples $\left(\mathcal{F}_{G}, \mathcal{F}_{T}, \kappa\right)$ for which each of the maps

$$
\mathcal{L}_{\mathcal{F}_{T}}^{\check{\lambda}} \rightarrow \mathcal{V}_{\mathcal{F}_{G}}^{\check{\lambda}}
$$

has a zero of order not less than (resp., exactly) $\langle\nu, \check{\lambda}\rangle$ at $x$, for all $\check{\lambda} \in \check{\Lambda}_{G}^{+}$.

By definition, $x, \geq 0 \overline{\operatorname{Bun}}_{B}=\overline{\operatorname{Bun}}_{B}$ and $\overline{\operatorname{Bun}}_{B}\left(\overline{\mathbb{F}_{q}}\right)=\underset{\nu \in \Lambda_{G}^{\text {pos }}}{x, \nu} \overline{\operatorname{Bun}}_{B}\left(\overline{\mathbb{F}_{q}}\right)$. We will denote by $j_{\nu}$ the embedding of ${ }_{x, \nu} \overline{\operatorname{Bun}}_{B}$ into $\overline{\operatorname{Bun}}_{B}$. It is easy to see that the map $i_{\nu}$ defines isomorphisms

$$
\overline{\operatorname{Bun}}_{B} \rightarrow{ }_{x, \geq \nu} \overline{\operatorname{Bun}}_{B} \text { and }{ }_{x, 0} \overline{\operatorname{Bun}}_{B} \rightarrow{ }_{x, \nu} \overline{\operatorname{Bun}}_{B}
$$


Let us consider in addition the open substack ${ }_{x, \nu} \operatorname{Bun}_{B} \subset{ }_{x, \nu} \overline{\operatorname{Bun}}_{B}$ that corresponds to triples $\left(\mathcal{F}_{G}, \mathcal{F}_{T}, \kappa\right)$ as above for which each of the maps

$$
\mathcal{L}_{\mathcal{F}_{T}}^{\check{\check{\lambda}}} \rightarrow \mathcal{V}_{\mathcal{F}_{G}}^{\check{\lambda}}
$$

has a zero of order exactly $\langle\nu, \check{\lambda}\rangle$ at $x$ and has no zeroes at other points of $X$. We have: ${ }_{x, 0} \operatorname{Bun}_{B}=\operatorname{Bun}_{B}$ and for $\nu \neq 0, i_{\nu}$ defines an isomorphism $\operatorname{Bun}_{B} \rightarrow{ }_{x, \nu} \operatorname{Bun}_{B}$.

By applying the decomposition theorem [2] (and taking into account the fact that $\phi_{!}\left(\mathrm{IC}_{\bar{Z}} \bar{Z}\right)$ is Verdier self-dual), we infer that Theorem 3.3.2 is equivalent to the following assertion:

Proposition 3.3.3. The complex $j_{\nu}^{*} \circ \phi_{!}\left(\mathrm{IC}_{x} \bar{Z}\right)$ satisfies the following:

(a) It lives in non-positive cohomological degrees (in the sense of the perverse $t$ structure).

(b) $h^{0}\left(j_{\nu}^{*} \circ \phi_{!}\left(\mathrm{IC}_{x} \bar{Z}\right)\right)$ identifies with $\mathrm{IC}_{x, \nu} \overline{\operatorname{Bun}}_{B} \otimes V^{\lambda}\left(w_{0}(\lambda)+\nu\right)$.

3.3.4. For an element $\nu \in \Lambda_{G}^{\text {pos }}$, let $Z^{\text {?, } \nu}$ (resp., $Z^{\nu, ?}$ ) denote the pre-image in ${ }_{x} \bar{Z}$ of the stratum ${ }_{x, \nu} \overline{\operatorname{Bun}}_{B} \subset \overline{\operatorname{Bun}}_{B}$ under the map ${ }^{\prime} h_{G}$ (resp., $\phi$ ). For two elements $\nu, \nu^{\prime} \in \Lambda_{G}^{\text {pos }}$ let $Z^{\nu, \nu^{\prime}}$ denote the intersection $Z^{\text {?, } \nu^{\prime}} \cap Z^{\nu, \text { ? }}$. (As we shall see shortly, $Z^{\nu, \nu^{\prime}}$ is empty unless $\nu \geq \nu^{\prime}$.)

If now $\lambda^{\prime}$ is an element of $\Lambda_{G}^{+}$such that $\lambda^{\prime} \leq \lambda$, let $Z^{\nu, \nu^{\prime}, \lambda^{\prime}}$ denote the intersection

$$
Z^{\nu, \nu^{\prime}} \cap \overline{\mathfrak{p}}^{-1}\left({ }_{x} \mathcal{H}_{G}^{\lambda^{\prime}}\right) \text {. }
$$

To prove Proposition 3.3.3 it is enough to prove the following

Proposition 3.3.5. For $\nu, \nu^{\prime}$ and $\lambda^{\prime}$ as above, let $K^{\nu, \nu^{\prime}, \lambda^{\prime}} \in \operatorname{Sh}\left({ }_{x, \nu} \overline{\operatorname{Bun}}_{B}\right)$ be defined as $\phi_{!}\left(\left.\mathrm{IC}_{x} \bar{Z}\right|_{Z^{\nu, \nu^{\prime}, \lambda^{\prime}}}\right)$. We have:

(a) $K^{\nu, \nu^{\prime}, \lambda^{\prime}}$ lives in cohomological degrees $\leq 0$ and the equality is strict unless $\nu^{\prime}=0$ and $\lambda^{\prime}=\lambda$.

(b) The *-restriction of $K^{\nu, 0, \lambda}$ to ${ }_{x, \nu} \overline{\operatorname{Bun}}_{B}-{ }_{x, \nu} \operatorname{Bun}_{B}$ lives in cohomological degrees $<0$.

(c) The restriction of $h^{0}\left(K^{\nu, 0, \lambda}\right)$ to ${ }_{x, \nu} \operatorname{Bun}_{B}$ can be identified with

$$
\mathrm{IC}_{x, \nu} \operatorname{Bun}_{B} \otimes V^{\lambda}\left(\mathrm{w}_{0}(\lambda)+\nu\right) .
$$

Consider the open sub-stack ${ }_{x, 0} \overline{\operatorname{Bun}}_{B} \subset \overline{\operatorname{Bun}}_{B}$. For $\left(\mathcal{F}_{G}, \mathcal{F}_{T}, \kappa\right) \in{ }_{x, 0} \overline{\operatorname{Bun}}_{B}$, the restriction of this data to the formal disc $\mathcal{D}_{x}$ defines a $B$-bundle over $\mathcal{D}_{x}$, or which is the same, a $B\left(\mathcal{O}_{x}\right)$-torsor. Therefore, there exists a canonical $B\left(\mathcal{O}_{x}\right)$-torsor over ${ }_{x, 0} \overline{\mathrm{Bun}}_{B}$, which we will denote by ${ }_{x} \mathcal{B}$.

Using the fact that $i_{\nu}$ maps ${ }_{x, 0} \overline{\mathrm{Bun}}_{B}$ isomorphically onto ${ }_{x, \nu} \overline{\mathrm{Bun}}_{B}$, we obtain a $B\left(\mathcal{O}_{x}\right)$-torsor ${ }_{x} \mathcal{B}^{\nu}$ over each ${ }_{x, \nu} \overline{\operatorname{Bun}}_{B}$. f We will denote by ${ }_{x} \stackrel{o}{\mathcal{B}}^{\nu}$ the restriction of ${ }_{x} \mathcal{B}^{\nu}$ to the open sub-set ${ }_{x, \nu} \operatorname{Bun}_{B} \subset{ }_{x, \nu} \overline{\operatorname{Bun}}_{B}$.

The next assertion follows from the definitions:

\footnotetext{
${ }^{4}$ Note, however, that there is no globally defined $B\left(\mathcal{O}_{x}\right)$-torsor over $\overline{\mathrm{Bun}}_{B}$ that would restrict to $\mathcal{B}^{\nu}$ over the corresponding stratum.
} 
Lemma 3.3.6. (a) The stack $Z^{?, \nu^{\prime}}$ with the projection ${ }^{\prime} h_{G}: Z^{?, \nu^{\prime}} \rightarrow_{x, \nu^{\prime}} \overline{\operatorname{Bun}}_{B}$ is a locally trivial fibration with the typical fiber $\overline{\mathrm{Gr}}_{G}^{-\mathrm{w}_{0}(\lambda)}$. More precisely there is an isomorphism

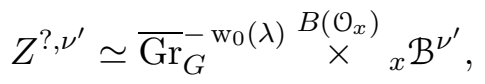

where $\overline{\mathrm{Gr}}_{G}^{-\mathrm{w}_{0}(\lambda)}$ is viewed as a $B\left(\mathcal{O}_{x}\right)$-scheme via $B\left(\mathcal{O}_{x}\right) \hookrightarrow G\left(\mathcal{O}_{x}\right)$.

(b) The sub-stack $Z^{\nu, \nu^{\prime}, \lambda^{\prime}} \hookrightarrow Z^{\text {?, } \nu^{\prime}}$ identifies (using the notation of point (a)) with

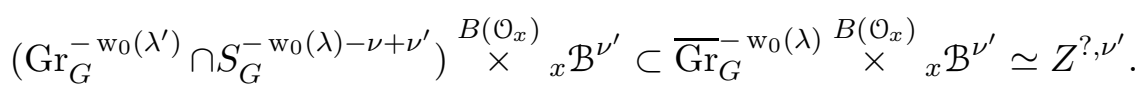

(c) The sub-stack $Z^{\nu^{\prime}, \nu, \lambda^{\prime}}$ when viewed as a stack projecting to ${ }_{x, \nu} \overline{\operatorname{Bun}}_{B}$ by means of $\phi$, identifies with

$$
\left(\operatorname{Gr}_{G}^{\lambda^{\prime}} \cap S_{G}^{\nu-\nu^{\prime}+\mathrm{w}_{0}(\lambda)}\right) \stackrel{B\left(\mathcal{O}_{x}\right)}{\times}{ }_{x} \mathcal{B}^{\nu} .
$$

(d) For every $\nu, \nu^{\prime}, \lambda^{\prime}$, the pre-image of the open substack ${ }_{x, \nu} \operatorname{Bun}_{B} \subset{ }_{x, \nu} \overline{\operatorname{Bun}}_{B}$ in $Z^{\nu^{\prime}, \nu, \lambda^{\prime}}$ under the projection $\phi$ coincides with the pre-image of ${ }_{x, \nu^{\prime}} \operatorname{Bun}_{B} \subset{ }_{x, \nu^{\prime}} \overline{\operatorname{Bun}}_{B}$ under ${ }^{\prime} h_{G}$.

3.3.7. We are finally ready to prove Proposition 3.3.5 (and hence Theorem 3.1.4):

Since the map $h_{G}:{ }_{x} \overline{\mathcal{H}}_{G}^{\lambda} \rightarrow \operatorname{Bun}_{G}$ is a locally trivial fibration, the sheaf $\left.\mathrm{IC}_{x} \bar{Z}\right|_{Z^{?}, \nu^{\prime}}$ is a twisted external product

$$
\left.\mathcal{A}_{G}^{-\mathrm{w}_{0}(\lambda)} \widetilde{\otimes} \mathrm{IC}_{\overline{\operatorname{Bun}}_{B}}\right|_{x, \nu^{\prime}} \overline{\operatorname{Bun}}_{B}
$$

(in the above identification we have used the isomorphism of Lemma 3.3.6(a)).

Hence, the restriction $\left.\mathrm{IC}_{x} \bar{Z}\right|_{Z^{\nu, \nu^{\prime}, \lambda^{\prime}}}$ is a twisted external product

$$
\left.\left.\mathcal{A}_{G}^{-\mathrm{w}_{0}(\lambda)}\right|_{\mathrm{Gr}_{G}^{-\mathrm{w}_{0}\left(\lambda^{\prime}\right)} \cap S_{G}^{-\mathrm{w}_{0}(\lambda)-\nu+\nu^{\prime}}} \widetilde{\otimes} \mathrm{IC}_{\overline{\mathrm{Bun}}_{B}}\right|_{x, \nu^{\prime}} \overline{\operatorname{Bun}}_{B} .
$$

First of all, by the very definition of IC, the sheaf $\left.\mathrm{IC}_{\overline{\mathrm{Bun}}_{B}}\right|_{x, \nu^{\prime}} \overline{\mathrm{Bun}}_{B}$ lives in cohomological degrees $\leq 0$ and the equality is strict unless $\nu^{\prime}=0$.

The restriction $\mathcal{A}_{G}^{-\mathrm{w}_{0}(\lambda)} \mid \operatorname{Gr}_{G}^{-\mathrm{w}_{0}\left(\lambda^{\prime}\right)}$ is a constant complex over $\operatorname{Gr}_{G}^{-\mathrm{w}_{0}\left(\lambda^{\prime}\right)}$ which lives in cohomological degrees $<0$ unless $\lambda^{\prime}=\lambda$. Using Proposition 3.2.6, we obtain that the sheaf

lives in cohomological degrees

$$
\mathcal{A}_{G}^{-\mathrm{w}_{0}(\lambda)} \mid \operatorname{Gr}_{G}^{-\mathrm{w}_{0}\left(\lambda^{\prime}\right)} \cap S_{G}^{-\mathrm{w}_{0}(\lambda)-\nu+\nu^{\prime}}
$$

$$
\leq-\operatorname{codim}\left(\mathrm{Gr}_{G}^{-\mathrm{w}_{0}\left(\lambda^{\prime}\right)} \cap S_{G}^{-\mathrm{w}_{0}\left(\lambda^{\prime}\right)-\nu+\nu^{\prime}}, \mathrm{Gr}_{G}^{-\mathrm{w}_{0}\left(\lambda^{\prime}\right)}\right) \leq-\left\langle\lambda^{\prime}-\lambda+\nu-\nu^{\prime}, \check{\rho}\right\rangle,
$$

with the inequality being strict unless $\lambda^{\prime}=\lambda$.

Hence, if either $\lambda^{\prime} \neq \lambda$ or $\nu^{\prime} \neq 0$, the sheaf $\left.\mathrm{IC}_{x} \bar{Z}\right|_{Z^{\nu, \nu^{\prime}}, \lambda^{\prime}}$ lives in cohomological degrees $<-\left\langle\lambda^{\prime}-\lambda+\nu-\nu^{\prime}, \check{\rho}\right\rangle$. However, Lemma 3.3.6.(c) and Proposition 3.2.6 imply that the dimension of the fibers of the projection

$$
\phi: Z^{\nu, \nu^{\prime}, \lambda^{\prime}} \rightarrow{ }_{x, \nu} \overline{\operatorname{Bun}}_{B}
$$


is $\leq\left\langle\lambda^{\prime}-\lambda+\nu-\nu^{\prime}, \check{\rho}\right\rangle$ and this proves point (a) of Proposition 3.3.5.

Let $Z^{\nu, 0, \lambda}$ denote the preimage of the open subset $\operatorname{Bun}_{B} \subset{ }_{x, 0} \overline{\operatorname{Bun}}_{B}$ in $Z^{\nu, 0, \lambda}$ under the map ' $h_{G}$. Considerations analogous to the ones above show that the restriction of $\left.\mathrm{IC}_{x} \bar{Z}\right|_{Z^{\nu, 0, \lambda}}$ to $Z^{\nu, 0, \lambda}-\stackrel{O}{Z}^{\nu, 0, \lambda}$ is concentrated in cohomological degrees $<0$. This implies Proposition 3.3.5(b) in view of Lemma 3.3.6(d).

To prove Proposition 3.3.5(c), observe that the restriction of $\left.\mathrm{IC}_{x} \bar{Z}\right|_{Z^{\nu, 0, \lambda}} ^{o}$ is a constant sheaf. More precisely, when we identify $\stackrel{o}{Z}^{\nu, 0, \lambda}$ (using points (c) and (d) of Lemma 3.3.6) with $\left(\mathrm{Gr}_{G}^{\lambda} \cap S_{G}^{\mathrm{w}_{0}(\lambda)+\nu}\right) \stackrel{B\left(\mathcal{O}_{x}\right)}{\times}{ }_{x} \stackrel{o}{\mathcal{B}}^{\nu}$, the sheaf $\left.\mathrm{IC}_{x} \bar{Z}\right|_{Z^{\nu, 0, \lambda}} ^{o}$ identifies with the twisted external product $\left(\overline{\mathbb{Q}_{\ell}}[1]\left(\frac{1}{2}\right)\right)_{\operatorname{Gr}_{G}^{\lambda} \cap S_{G}^{\mathrm{w}_{0}(\lambda)+\nu}}^{\otimes\langle\nu, 2 \check{\otimes}} \mathrm{IC}_{x, \nu}$ Bun.

As the group $B\left(\mathcal{O}_{x}\right)$ is connected, this implies that the 0 -th=top perverse cohomology of its direct image on ${ }_{x, \nu}$ Bun can be identified with

$$
\mathrm{IC}_{x, \nu} \operatorname{Bun} \otimes H_{c}^{0}\left(\operatorname{Gr}_{G}^{\lambda} \cap S_{G}^{\mathrm{w}_{0}(\lambda)+\nu},\left(\overline{\mathbb{Q}_{\ell}}[1]\left(\frac{1}{2}\right)\right)^{\otimes\langle\nu, 2 \check{\rho}\rangle}\right) .
$$

Now, the assertion of Proposition 3.3.5(c) follows from Corollary 3.2.9(b).

\section{Action of Hecke operators (the non-principal Case)}

\subsection{Variant of the basic diagram.}

4.1.1. Let $P$ be a parabolic sub-group of $G$. Consider the stack ${ }_{x, \infty} \widetilde{\operatorname{Bun}}_{P}$ that classifies triples

$$
\left(\mathcal{F}_{G}, \mathcal{F}_{M}, \widetilde{\kappa}_{P}:\left.\mathcal{F}_{G}\right|_{X-x} \rightarrow \overline{G / U(P)} \times\left.\mathcal{F}_{M}\right|_{X-x}\right) .
$$

In other words, a point of $x, \widetilde{\operatorname{Bun}}_{P}$ is a pair $\left(\mathcal{F}_{G}, \mathcal{F}_{M}\right) \in \operatorname{Bun}_{G} \times \operatorname{Bun}_{M}$ and a compatible system of maps

$$
\widetilde{\kappa}_{P}^{\mathcal{V}}:\left(\mathcal{V}^{U(P)}\right)_{\mathcal{F}_{M}} \rightarrow \mathcal{V}_{\mathcal{F}_{G}}(\infty \cdot x),
$$

for every $G$-module $\mathcal{V}$, which satisfy the Plücker relations in the same sense as in the definition of $\widetilde{\operatorname{Bun}}_{P}$ (cf. Sect. 1.3.5).

By bounding degrees of poles of the maps $\widetilde{\kappa}_{P}^{\mathcal{V}}$ we can represent ${ }_{x, \infty} \widetilde{\operatorname{Bun}} P$ as a union of its finite-dimensional closed substacks:

Let $\nu \in \Lambda$ be dominant with respect to $M$, i.e. $\nu \in \Lambda_{M}^{+}$. We define a closed finitedimensional substack ${ }_{x, \geq \nu} \widetilde{\operatorname{Bun}_{P}}$ as follows: it corresponds to those triples $\left(\mathcal{F}_{G}, \mathcal{F}_{M}, \widetilde{\kappa}_{P}\right)$ for which $\widetilde{\kappa}_{P}^{\mathcal{V}}$ maps

$$
\left(\mathcal{V}^{U(P)}\right)_{\mathcal{F}_{M}} \rightarrow \mathcal{V}_{\mathcal{F}_{G}}\left(\left\langle-\mathrm{w}_{0}^{M}(\nu), \check{\lambda}\right\rangle \cdot x\right),
$$

if $\mathcal{V}$ is a $G$-module whose weights are $\leq \check{\lambda}$. Let $i_{\nu}$ denote the corresponding closed embedding.

In particular, $\widetilde{\operatorname{Bun}}_{P} \subset{ }_{x, \infty} \widetilde{\operatorname{Bun}}_{P}$ coincides with ${ }_{x, \geq 0} \widetilde{\operatorname{Bun}}_{P}$. It is clear that if for $\nu^{\prime} \in \Lambda_{M}^{+}, \mathrm{w}_{0}^{M}\left(\nu^{\prime}-\nu\right) \in \Lambda_{G}^{\mathrm{pos}}$, then ${ }_{x, \geq \nu^{\prime}} \widetilde{\operatorname{Bun}}_{P}$ is contained in ${ }_{x, \geq \nu} \widetilde{\operatorname{Bun}}_{P}$ and that ${ }_{x, \infty} \widetilde{\operatorname{Bun}}_{P}$ is the inductive limit of the ${ }_{x, \geq \nu} \widetilde{\operatorname{Bun}}_{P}$ 's. 
We will abuse the notation and continue to denote by $\widetilde{\mathfrak{p}}_{P}$ and $\widetilde{\mathfrak{q}}_{P}$ the natural projections from ${ }_{x, \infty} \widetilde{\operatorname{Bun}}_{P}$ to $\operatorname{Bun}_{G}$ and to $\operatorname{Bun}_{M}$, respectively. Finally, note that when $P=G$, the stack ${ }_{x, \infty} \widetilde{\operatorname{Bun}}_{P}$ is identified with the Hecke stack ${ }_{x} \mathcal{H}_{G}$.

4.1.2. Let ${ }_{x, \infty} Z_{P, M}$ denote the Cartesian product:

$$
{ }_{x, \infty} Z_{P, M}={ }_{x} \mathcal{H}_{M} \underset{\operatorname{Bun}_{M}}{\times} x, \widetilde{\operatorname{Bun}}_{P},
$$

where ${ }_{x} \mathcal{H}_{M}$ maps to $\operatorname{Bun}_{M}$ by means of $h_{M}$.

By definition, the stack ${ }_{x, \infty} Z_{P, M}$ classifies quadruples $\left(\mathcal{F}_{G}, \mathcal{F}_{M}, \mathcal{F}_{M}^{\prime}, \widetilde{\kappa}_{P}\right)$, where $\left(\mathcal{F}_{G}, \mathcal{F}_{M}^{\prime}, \widetilde{\kappa}_{P}\right)$ is a point of ${ }_{x, \infty} \widehat{\operatorname{Bun}}_{P}$ and $\mathcal{F}_{M}$ is an $M$-bundle over $X$ identified with $\mathcal{F}_{M}^{\prime}$ over $X-x$. However, to a quadruple $\left(\mathcal{F}_{G}, \mathcal{F}_{M}, \mathcal{F}_{M}^{\prime}, \widetilde{\kappa}_{P}\right)$ as above one can attach another point of ${ }_{x, \infty} \widetilde{\operatorname{Bun}}_{P}$, namely $\left(\mathcal{F}_{G}, \mathcal{F}_{M}, \widetilde{\kappa}_{P}\right)$. Thus, as in Sect. 3.1.1 we obtain a second projection ' $h_{M}^{\leftarrow}:{ }_{x, \infty} Z_{P, M} \rightarrow \operatorname{Bun}_{M}$

It is easy to see that we obtain in fact two Cartesian squares:

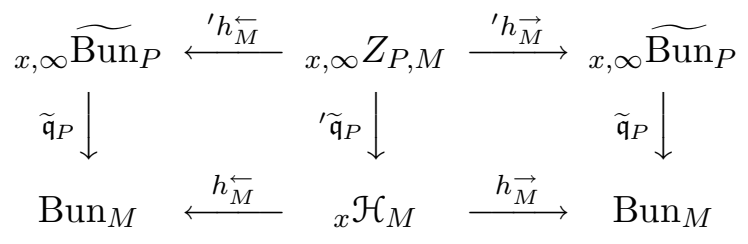

Therefore, for $\mathcal{S} \in \operatorname{Sph}_{M}$ and $\mathcal{T} \in \operatorname{Sh}\left({ }_{x, \infty} \widetilde{\operatorname{Bun}}_{P}\right)$ we can define their twisted external products $(\mathcal{S} \widetilde{\otimes} \mathcal{T})^{l},(\mathcal{S} \widetilde{\otimes} \mathcal{T})^{r} \in \operatorname{Sh}\left({ }_{x, \infty} \widetilde{\operatorname{Bun}_{P}}\right)$. We introduce the Hecke functors ${ }_{x} H_{P, M}^{\leftarrow}(\cdot, \cdot)$ and ${ }_{x} H_{P, M}(\cdot, \cdot)$ from $\operatorname{Sph}_{M} \times \operatorname{Sh}\left({ }_{x, \infty} \widetilde{\operatorname{Bun}}_{P}\right)$ to $\operatorname{Sh}\left({ }_{x, \infty} \widetilde{\operatorname{Bun}}_{P}\right)$ by setting

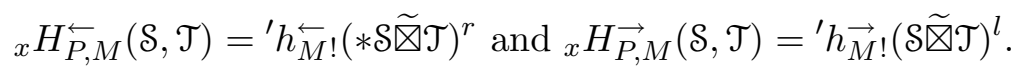

These functors have the properties exactly analogous to those of ${ }_{x} H_{G}^{\leftarrow}(\cdot, \cdot)$ and ${ }_{x} H_{G}(\cdot, \cdot)$, described in Sect. 3.2.4.

For $\nu \in \Lambda_{M}^{+}$, we will denote by ${ }_{x} H_{P, M}^{\nu}$ the self-functor on $\operatorname{Sh}\left({ }_{x, \infty} \widetilde{\operatorname{Bun}}_{P}\right)$ defined as $\mathcal{T} \rightarrow{ }_{x} H_{P, M}^{\leftarrow}\left(\mathcal{A}_{M}^{\nu}, \mathcal{T}\right)$

We have the following assertion:

Theorem 4.1.3. ${ }_{x} H_{P, M}^{\nu}\left(\mathrm{IC}_{x, \geq 0}{\widetilde{\operatorname{Bu}_{P}}}_{P}\right)$ is canonically isomorphic to the intersection cohomology sheaf of $x, \geq-w_{0}^{M}(\nu) \widehat{\operatorname{Bun}}_{P}$.

A proof of this theorem will be given in the next subsection.

4.1.4. Now let us consider the stack

$$
{ }_{x, \infty} Z_{P, G}={ }_{x} \mathcal{H}_{G} \underset{\operatorname{Bun}_{G} x, \infty}{\times \widetilde{\operatorname{Bun}}_{P}}
$$

where ${ }_{x} \mathcal{H}_{G}$ projects to $\operatorname{Bun}_{G}$ by means of $h_{G}$.

Similarly to what we had above, one can define a second projection ' $h_{G}^{\leftarrow}:{ }_{x, \infty} Z_{P, G} \rightarrow$ $\operatorname{Bun}_{G}$ and we obtain a diagram 
in which both squares are Cartesian.

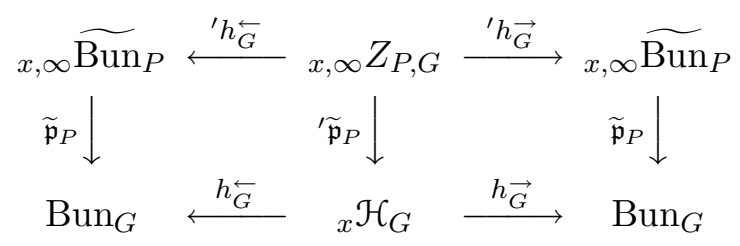

Proceeding as above, we obtain two functors ${ }_{x} H_{P, G}^{\leftarrow}(\mathcal{S}, \mathcal{T})$ and $\left.{ }_{x} H_{P, G} \rightarrow \mathcal{S}, \mathcal{T}\right)$ from $\operatorname{Sph}_{G} \times \operatorname{Sh}\left({ }_{x, \infty} \widetilde{\operatorname{Bun}}_{P}\right) \mapsto \operatorname{Sh}\left({ }_{x, \infty} \widetilde{\operatorname{Bun}}_{P}\right)$. For $\lambda \in \Lambda_{G}^{+}$and $\mathcal{T} \in \operatorname{Sh}\left({ }_{x, \infty} \widetilde{\operatorname{Bun}}_{P}\right)$ we set ${ }_{x} H_{P, G}^{\lambda}(\mathcal{T})={ }_{x} H_{P, G}^{\leftarrow}\left(\mathcal{A}_{G}^{\lambda}, \mathcal{T}\right)$.

It is easy to see that for $?=\rightarrow$ or $\leftarrow$ and $?^{\prime}=\rightarrow$ or $\leftarrow$ the actions ${ }_{x} H_{P, G}^{?}$ and ${ }_{x} H_{P, M}^{?^{\prime}}$ commute in the sense that there is a functorial isomorphism

$$
{ }_{x} H_{P, G}^{?}\left(\mathcal{S}_{G},{ }_{x} H_{P, M}^{?^{\prime}}\left(\mathcal{S}_{M}, \mathcal{T}\right)\right) \simeq{ }_{x} H_{P, M}^{?}\left(\mathcal{S}_{M},{ }_{x} H_{P, G}^{?^{\prime}}\left(\mathcal{S}_{G}, \mathcal{T}\right)\right),
$$

where $\mathcal{S}_{G} \in \operatorname{Sph}_{G}, \mathcal{S}_{M} \in \operatorname{Sph}_{M}$ and $\mathcal{T} \in \operatorname{Sh}\left({ }_{x, \infty} \widetilde{\operatorname{Bun}}_{P}\right)$.

Theorem 4.1.5. For $\lambda \in \Lambda_{G}^{+}$there is a canonical isomorphism:

$$
{ }_{x} H_{P, G}^{\lambda}\left(\mathrm{IC}_{x, \geq 0} \widetilde{\operatorname{Bun}_{P}}\right) \simeq \underset{\nu \in \Lambda_{M}^{+}}{\oplus} \mathrm{IC}_{x, \geq \nu} \widetilde{\operatorname{Bun}_{P}} \otimes \operatorname{Hom}_{\check{M}}\left(U^{\nu}, V^{\lambda}\right) .
$$

4.1.6. The combination of Theorem 4.1.5 and Theorem 4.1.3 yields the following:

Let $\operatorname{gRes}_{M}^{G}: \operatorname{Sph}_{G} \rightarrow \operatorname{Sph}_{M}$ denote the tensor functor corresponding to the restriction functor $\operatorname{Res}_{M}^{G}: \operatorname{Rep}(\check{G}) \rightarrow \operatorname{Rep}(\check{M})$ under the equivalence of Theorem 3.2.8.

Corollary 4.1.7. The two functors $\operatorname{Sph}_{G} \rightarrow \operatorname{Sh}\left(x, \infty \widetilde{\operatorname{Bun}}_{P}\right)$ :

$$
\mathcal{S} \mapsto{ }_{x} H_{P, G}^{\leftarrow}\left(\mathcal{S}, \mathrm{IC}_{x, \geq 0} \widetilde{\operatorname{Bun}_{P}}\right) \text { and } \mathcal{S} \mapsto{ }_{x} H_{P, M}\left(\operatorname{gRes}_{M}^{G}(\mathcal{S}), \mathrm{IC}_{x, \geq 0} \widetilde{\operatorname{Bun}_{P}}\right)
$$

are canonically isomorphic.

Moreover, from the proof of the above theorems, it will follow that the isomorphism of functors of the above corollary is compatible with the tensor structure in the following sense:

Let $\mathcal{S}_{1}, \mathcal{S}_{2} \in \operatorname{Sph}_{G}$ the two isomorphisms

$$
\begin{aligned}
& { }_{x} H_{P, G}^{\leftarrow}\left(\mathcal{S}_{1},{ }_{x} H_{P, G}^{\leftarrow}\left(\mathcal{S}_{2}, \mathrm{IC}_{\overparen{\operatorname{Bun}_{P}}}\right)\right) \simeq{ }_{x} H_{P, G}^{\leftarrow}\left(\mathcal{S}_{1} \star \mathcal{S}_{2}, \mathrm{IC}_{\mathrm{Bun}_{P}}\right) \rightarrow \\
& { }_{x} H_{P, M}^{\rightarrow}\left(\operatorname{gRes}_{M}^{G}\left(\mathcal{S}_{1} \star \mathcal{S}_{2}\right), \mathrm{IC}_{\widetilde{\operatorname{Bun}_{P}}}\right) \rightarrow{ }_{x} H_{P, M}\left(\operatorname{gRes}_{M}^{G}\left(\mathcal{S}_{1}\right) \star \operatorname{gRes}_{M}^{G}\left(\mathcal{S}_{2}\right), \operatorname{IC}_{\widetilde{\operatorname{Bun}_{P}}}\right) \rightarrow \\
& { }_{x} H_{P, M}^{\rightarrow}\left(\operatorname{gRes}_{M}^{G}\left(\mathcal{S}_{2}\right),{ }_{x} H_{P, M}^{\rightarrow}\left(\operatorname{gRes}_{M}^{G}\left(\mathcal{S}_{1}\right), \mathrm{IC}_{\widetilde{\operatorname{Bun}_{P}}}\right)\right)
\end{aligned}
$$

and

$$
\begin{aligned}
& \left.{ }_{x} H_{P, G}^{\leftarrow}\left(\mathcal{S}_{1},{ }_{x} H_{P, G}^{\leftarrow}\left(\mathcal{S}_{2}, \mathrm{IC}_{\widetilde{\mathrm{Bun}_{P}}}\right)\right) \rightarrow{ }_{x} H_{P, G}^{\leftarrow}\left(\mathcal{S}_{1},{ }_{x} H_{P, M} \rightarrow \operatorname{gRes}_{M}^{G}\left(\mathcal{S}_{2}\right), \mathrm{IC}_{\widetilde{\operatorname{Bun}_{P}}}\right)\right) \rightarrow \\
& { }_{x} H_{P, M}^{\rightarrow}\left(\operatorname{gRes}_{M}^{G}\left(\mathcal{S}_{2}\right),{ }_{x} H_{P, G}^{\leftarrow}\left(\mathcal{S}_{1}, \mathrm{IC}_{\widetilde{\mathrm{Bun}_{P}}}\right)\right) \rightarrow \\
& { }_{x} H_{P, M}^{\vec{P}}\left(\operatorname{gRes}_{M}^{G}\left(\mathcal{S}_{2}\right),{ }_{x} H_{P, M}^{\vec{P}}\left(\operatorname{gRes}_{M}^{G}\left(\mathcal{S}_{1}\right), \mathrm{IC}_{\mathrm{Bun}_{P}}\right)\right)
\end{aligned}
$$

coincide. We leave the verification to the reader. 
4.1.8. Now let us explain how Theorem 4.1.5 implies Theorem 2.3.7.

To simplify the notation, we shall prove the following statement: For $\mathcal{S} \in \operatorname{Sph}_{G}$ and $\mathcal{T} \in \operatorname{Sh}\left(\operatorname{Bun}_{M}\right)$ there is a functorial isomorphism

$$
{ }_{x} H_{G}^{\leftarrow}\left(\mathcal{S}, \operatorname{Eis}_{M}^{G}(\mathcal{T})\right) \simeq \operatorname{Eis}_{M}^{G}\left({ }_{x} H_{M}^{\leftarrow}\left(\operatorname{gRes}_{M}^{G}(\mathcal{S}), \mathcal{T}\right)\right) .
$$

Moreover, this isomorphism is compatible with the tensor structures on $\mathrm{Sph}_{G}$ and on $\mathrm{Sph}_{M}$.

The proof of Theorem 2.3.7 in its original form (i.e. when $x$ is not fixed) is absolutely analogous.

For $\mathcal{T} \in \operatorname{Sh}\left(\operatorname{Bun}_{M}\right)$ the LHS of (1) can be rewritten using the stack ${ }_{x, \infty} \widetilde{\operatorname{Bun}}_{P}$ instead of $\widetilde{\operatorname{Bun}}_{P}$ :

$$
{ }_{x} H_{G}^{\leftarrow}\left(\mathcal{S}, \operatorname{Eis}_{M}^{G}(\mathcal{T})\right):={ }_{x} H_{G}^{\leftarrow}\left(\mathcal{S}, \widetilde{\mathfrak{p}}_{P !}\left(\widetilde{\mathfrak{q}}_{P}^{*}(\mathcal{T}) \otimes \mathrm{IC}_{x, \geq 0} \widetilde{\operatorname{Bun}_{P}}\right)\right) \otimes\left(\overline{\mathbb{Q}_{\ell}}[1]\left(\frac{1}{2}\right)\right)^{\otimes-\operatorname{dim}\left(\operatorname{Bun}_{M}\right)} .
$$

The maps $\widetilde{\mathfrak{q}}_{P} \circ^{\prime} h_{G}^{\overleftarrow{G}}$ and $\widetilde{\mathfrak{q}}_{P} \circ^{\prime} h_{G}$ from ${ }_{x, \infty} Z_{P, G}$ to Bun $_{M}$ coincide. Therefore, using the projection formula and the base change on the diagram definining ${ }_{x, \infty} Z_{P, G}$, the above expression can be rewritten as

$$
\widetilde{\mathfrak{p}}_{P !}\left(\widetilde{\mathfrak{q}}_{P}^{*}(\mathcal{T}) \otimes{ }_{x} H_{P, G}^{\leftarrow}\left(\mathcal{S}, \mathrm{IC}_{x, \geq 0} \widetilde{\operatorname{Bun}_{P}}\right)\right) \otimes\left(\overline{\mathbb{Q}}[1]\left(\frac{1}{2}\right)\right)^{\otimes-\operatorname{dim}\left(\operatorname{Bun}_{M}\right)}
$$

Now, let us apply Theorem 4.1.5 (in the form of Corollary 4.1.7) and rewrite (2) as

$$
\widetilde{\mathfrak{p}}_{P !}\left(\widetilde{\mathfrak{q}}_{P}^{*}(\mathcal{T}) \otimes{ }_{x} H_{P, M} \rightarrow\left(\operatorname{gRes}_{M}^{G}(\mathcal{S}), \mathrm{IC}_{x, \geq 0} \widetilde{\operatorname{Bun}_{P}}\right)\right) \otimes\left(\overline{\mathbb{Q}_{\ell}}[1]\left(\frac{1}{2}\right)\right)^{\otimes-\operatorname{dim}\left(\operatorname{Bun}_{M}\right)} .
$$

Again, since $\widetilde{\mathfrak{p}}_{P} \circ^{\prime} h_{M}^{\overleftarrow{ }}=\widetilde{\mathfrak{p}}_{P} \circ^{\prime} h_{M}$ as maps ${ }_{x, \infty} Z_{P, M} \rightarrow$ Bun $_{G}$, the projection formula implies that (4.1.8) is the same as

$$
\widetilde{\mathfrak{p}}_{P !}\left(\widetilde{\mathfrak{q}}_{P}^{*}\left({ }_{x} H_{M}^{\leftarrow}\left(\operatorname{gRes}_{M}^{G}(\mathcal{S}), \mathcal{T}\right)\right) \otimes \mathrm{IC}_{x, \geq 0} \widetilde{\operatorname{Bun}_{P}}\right) \otimes\left(\overline{\mathbb{Q}_{\ell}}[1]\left(\frac{1}{2}\right)\right)^{\otimes-\operatorname{dim}\left(\operatorname{Bun}_{M}\right)}
$$

which is what we had to prove.

The fact that this system of isomorphisms is compatible with the tensor structure follows from corresponding property of the isomorphism of Corollary 4.1.7.

\subsection{Proof of Theorem 4.1.3.}

4.2.1. Let ${ }_{x, 0} \widetilde{\operatorname{Bun}}_{P}$ denote the open substack of $x_{x, \geq 0} \widetilde{\operatorname{Bun}}_{P}=\widetilde{\operatorname{Bun}}_{P}$ that corresponds to those triples $\left(\mathcal{F}_{G}, \mathcal{F}_{M}, \widetilde{\kappa}_{P}\right)$ for which the maps

$$
\widetilde{\kappa}_{P}^{\mathcal{V}}:\left(\mathcal{V}^{U(P)}\right)_{\mathcal{F}_{M}} \rightarrow \mathcal{V}_{\mathcal{F}_{G}}
$$

have no zero at $x$.

Proposition 4.2.2. The composition

$$
{ }^{\prime} h_{M}^{-1}\left({ }_{x, 0} \widetilde{\operatorname{Bun}}_{P}\right) \hookrightarrow{ }_{x, \infty} Z_{P, M} \stackrel{{ }^{\prime} h_{M}}{\longrightarrow} x, \infty \widetilde{\operatorname{Bun}}_{P}
$$

is a locally closed embedding. 
Proof. It is easy to see that the assertion of the proposition amounts to the following:

Let $\left(\mathcal{F}_{G}, \mathcal{F}_{M}, \widetilde{\kappa}_{P}\right)$ be as $S$-point of $\widetilde{\operatorname{Bun}}_{P}$, such that for every $G$-module $\mathcal{V}$, the sheaf embedding

$$
\widetilde{\kappa}^{\mathcal{V}}:\left(\mathcal{V}^{U(P)}\right)_{\mathcal{F}_{M}} \rightarrow \mathcal{V}_{\mathcal{F}_{G}}
$$

has no zero along the divisor $x \times S \subset X \times S$. Suppose that $\left(\mathcal{F}_{M}, \mathcal{F}_{M}^{\prime}, \beta_{M}\right)$ is an $S$-point of ${ }_{x} \mathcal{H}_{M}$ such that the a priori meromorphic maps

$$
\widetilde{\kappa}^{\prime \mathcal{V}}:\left.\left.\left(\mathcal{V}^{U(P)}\right)_{\mathcal{F}_{M}^{\prime}}\right|_{(X-x) \times S} \rightarrow \mathcal{V}_{\mathcal{F}_{G}}\right|_{(X-x) \times S}
$$

extend to regular maps on $X \times S$, which do not have zeroes along $x \times S$.

In this case we have to show that $\mathcal{F}_{M}^{\prime}$ in fact equals $\mathcal{F}_{M}$, i.e. that $\beta_{M}$ induces regular maps $\beta_{M}^{\mathcal{U}}: \mathcal{U}_{\mathcal{F}_{M}^{\prime}} \rightarrow \mathcal{U}_{\mathcal{F}_{M}}$ for every $M$-module $\mathcal{U}$.

First, let us assume that $\mathcal{U}$ is isomorphic to $\mathcal{V}^{U(P)}$ for some $G$-module $\mathcal{V}$. In this case, the above map $\widetilde{\kappa}^{\prime \mathcal{V}}$ must factor as

$$
\left(\mathcal{V}^{U(P)}\right)_{\mathcal{F}_{M}^{\prime}} \stackrel{\beta_{M}^{\mathfrak{u}}}{\longrightarrow}\left(\mathcal{V}^{U(P)}\right)_{\mathcal{F}_{M}} \stackrel{\widetilde{\kappa}^{\mathcal{V}}}{\longrightarrow} \mathcal{V}_{\mathcal{F}_{G}}
$$

because $\left(\mathcal{V}^{U(P)}\right)_{\mathcal{F}_{M}}$ is maximal at $x \times S$ in $\mathcal{V}_{\mathcal{F}_{G}}$, by assumption. In particular, this means that $\beta_{M}^{\mathcal{U}}$ is regular.

The same argument shows that if $\mathcal{U}$ is 1-dimensional, corresponding to a character $\check{\lambda} \in \check{\Lambda}_{G, P} \cap \Lambda_{G}^{+}$, the map $\beta_{M}^{\mathcal{U}}$ is an isomorphism. Since the semigroup $\check{\lambda} \in \check{\Lambda}_{G, P}$ generates $\check{\Lambda}_{G, P}$, we obtain that $\beta_{M}^{\mathrm{U}}$ is an isomorphism for all 1-dimensional representations.

To finish the proof, it remains to observe that every $M$-module can be embedded into a tensor product of a 1-dimensional module and a one of the form $\mathcal{V}^{U(P)}$.

For $\nu \in \Lambda_{M}^{+}$, let $\widetilde{\operatorname{Bun}}_{P}$ denote the image under ' $h_{M}^{-1}\left(_{x, 0} \widetilde{\operatorname{Bun}}_{P}\right){\stackrel{ }{\prime} h_{M}}_{x, \infty} \widetilde{\operatorname{Bun}}_{P}$ of ${ }^{\prime} h_{M} \overleftarrow{1}^{-1}\left({ }_{x, 0} \widetilde{\operatorname{Bun}}_{P}\right) \cap \widetilde{\mathfrak{q}}_{P}^{-1}\left({ }_{x} \mathcal{H}_{M}^{\nu}\right)$. Let $j_{\nu}$ denote the locally closed embedding of ${ }_{x, \nu} \widetilde{\operatorname{Bun}}_{P}$ into $x, \infty \widetilde{\operatorname{Bun}}_{P}$.

Proposition 4.2.3. The locally closed substacks ${ }_{x, \nu^{\prime}} \widetilde{\operatorname{Bun}}_{P}, \mathrm{w}_{0}^{M}\left(\nu^{\prime}-\nu\right) \in \Lambda_{G}^{\mathrm{pos}}$ form a stratification of $x_{x, \geq \nu} \widetilde{\operatorname{Bun}}_{P}$, with ${ }_{x, \nu} \widetilde{\operatorname{Bun}}_{P}$ being the biggest stratum.

Proof. The fact that ${ }_{x, \nu} \widetilde{\operatorname{Bun}}_{P}$ is contained in ${ }_{x, \geq \nu} \widetilde{\operatorname{Bun}}_{P}$ follows from the definitions. Therefore, to prove the proposition we have to show that every $\overline{\mathbb{F}_{q}}$-point $\left(\mathcal{F}_{G}, \mathcal{F}_{M}, \widetilde{\kappa}_{P}\right)$ of ${ }_{x, \infty} \widetilde{\operatorname{Bun}}_{P}$ is contained is some ${ }_{x, \geq \nu} \widetilde{\operatorname{Bun}}_{P}$.

The latter amounts to showing that there exists an $M$-bundle $\mathcal{F}_{M}^{\prime}$ and an isomorphism $\beta_{M}:\left.\left.\mathcal{F}_{M}^{\prime}\right|_{X-x} \rightarrow \mathcal{F}_{M}\right|_{X-x}$, such that the composition

$$
\left.\left.\left.\left(\mathcal{V}^{U(P)}\right)_{\mathcal{F}_{M}^{\prime}}\right|_{X-x} \stackrel{\beta_{M}^{\mathcal{L}}}{\longrightarrow}\left(\mathcal{V}^{U(P)}\right)_{\mathcal{F}_{M}}\right|_{X-x} \stackrel{\widetilde{\kappa}^{\mathcal{V}}}{\longrightarrow} \mathcal{V}_{\mathcal{F}_{G}}\right|_{X-x}
$$

extends to a regular map on the entire $X$, which has no zeroes at $x$.

The construction of such $\mathcal{F}_{M}^{\prime}$ repeats the proof of Proposition 1.2.5 in its parabolic variant. 
Since the complex ${ }_{x} H_{P, M}^{\nu}\left(\mathrm{IC}_{\widetilde{\mathrm{Bun}_{P}}}\right)$ is self-dual, in order to prove Theorem 4.1.3 it suffices to prove the following assertion:

Proposition 4.2.4. For any $\nu^{\prime} \in \Lambda_{M}^{+}$the complex

$$
j_{\nu^{\prime}}^{*} \circ h_{M !}^{\leftarrow}\left(\mathcal{A}_{M}^{-\mathrm{w}_{0}^{M}(\nu)} \widetilde{\otimes} \mathrm{IC}_{\widetilde{\mathrm{Bun}_{P}}}\right)^{r}
$$

lives in cohomological degrees $\leq 0$ and the inequality is strict unless $\nu^{\prime}=-\mathrm{w}_{0}^{M}(\nu)$. Moreover, for $\nu^{\prime}=-\mathrm{w}_{0}^{M}(\nu)$ its 0 -th cohomology can be identified with $\mathrm{IC}_{x,-\mathrm{w}_{0}^{M}(\nu)} \widetilde{\operatorname{Bun}_{P}}$

4.2.5. For $\nu^{\prime}, \eta, \eta^{\prime} \in \Lambda_{M}^{+}$let us consider the following locally closed sub-stacks of $x, \infty Z_{P, M}$ :

$$
\begin{aligned}
& Z_{P, M}^{\nu^{\prime}, ?, ?}:=\left({ }^{\prime} h_{M}^{\overleftarrow{ }}\right)^{-1}\left({ }_{x, \nu^{\prime}} \widetilde{\operatorname{Bun}}_{P}\right), Z_{P, M}^{?, \eta, ?}:=\left({ }^{\prime} h_{M}\right)^{-1}\left({ }_{x, \eta} \widetilde{\operatorname{Bun}}_{P}\right) \\
& Z_{P, M}^{?, ?, \eta^{\prime}}:=\left({ }^{\prime} \widetilde{\mathfrak{q}}_{P}\right)^{-1}\left({ }_{x} \mathcal{H}_{M}^{\eta^{\prime}}\right) .
\end{aligned}
$$

Let $Z_{P, M}^{\nu^{\prime}, \eta, \eta^{\prime}}$ denote their intersection and similarly for $Z_{P, M}^{?, \eta, \eta^{\prime}}$, etc.

We will denote by $K_{M}^{\nu^{\prime}, \eta, \eta^{\prime}}$ the complex

$$
{ }^{\prime} h_{M !}^{\overleftarrow{ } !}\left(\left.\left(\mathcal{A}_{M}^{-\mathrm{w}_{0}^{M}(\nu)} \widetilde{\otimes} \mathrm{IC}_{\widetilde{\mathrm{Bun}_{P}}}\right)^{r}\right|_{Z_{P, M}^{\nu^{\prime}, \eta, \eta^{\prime}}}\right)
$$

on $\widetilde{\operatorname{Bun}}_{P}$.

To prove the proposition it is enough to show that $K_{M}^{\nu^{\prime}, \eta, \eta^{\prime}}$ lives in negative cohomological degrees unless $\nu^{\prime}=-\mathrm{w}_{0}^{M}(\nu), \eta=0, \eta^{\prime}=\nu$ and that

$$
h^{0}\left(K_{M}^{-\mathrm{w}_{0}^{M}(\nu), 0, \nu}\right) \simeq \mathrm{IC}_{x,-\mathrm{w}_{0}^{M}(\nu)} \widetilde{\operatorname{Bun}_{P}} .
$$

Recall the indscheme $\operatorname{Conv}_{M}$ (cf. Sect. 3.2.2). As we have seen before, the map $p r^{\prime}$ makes it a fibration over $\operatorname{Gr}_{M}$ with the typical fiber $\operatorname{Gr}_{M}$. For $\nu_{1}, \nu_{2} \in \Lambda_{M}^{+}$let $\operatorname{Conv}_{M}^{\nu_{1}, \nu_{2}}$ be the corresponding locally closed subscheme of $\operatorname{Conv}_{M}$, which is fibered over $\operatorname{Gr}_{M}^{\nu_{1}}$ with the typical fiber being $\mathrm{Gr}_{M}^{\mu_{2}}$. If $\mu_{3}$ is a third element of $\Lambda_{M}^{+}$, we will denote by $\operatorname{Conv}_{M}^{\mu_{1}, \mu_{2} ; \mu_{3}}$ the intersection $\operatorname{Conv}_{M}^{\nu_{1}, \nu_{1}} \cap p^{-1}\left(\operatorname{Gr}_{M}^{\nu_{3}}\right)$.

We will need the following fact, which follows easily from Proposition 3.2.3 (or alternatively, from Proposition 3.2.6):

$$
\operatorname{dim}\left(\operatorname{Conv}_{M}^{\mu_{1}, \mu_{2} ; \mu_{3}}\right)=\left\langle\mu_{1}+\mu_{2}+\mu_{3}, \check{\rho}_{M}\right\rangle
$$

Consider the composition

$$
Z_{P, M}^{\nu^{\prime}, ?, \eta^{\prime}}{\stackrel{\prime h_{M}^{\leftarrow}}{\longrightarrow}}_{x, \nu^{\prime}} \widetilde{\operatorname{Bun}}_{P} \simeq \widetilde{\operatorname{Bun}}_{P} \underset{\operatorname{Bun}_{M}}{\times} \mathcal{H}_{M}^{\nu^{\prime}} \rightarrow \widetilde{\operatorname{Bun}}_{P}
$$

It follows from the definitions that $Z_{P, M}^{\nu^{\prime}, ?, \eta^{\prime}}$ is a locally trivial fibration over $\widetilde{\mathrm{Bun}}_{P}$ with the typical fiber $\operatorname{Conv}_{M}^{\nu^{\prime}, \eta^{\prime}}$. More precisley, we obtain the following identifications of 
stacks:

$$
\begin{gathered}
Z_{P, M}^{\nu^{\prime}, ?, \eta^{\prime}} \stackrel{\sim}{\longrightarrow} \operatorname{Conv}_{M}^{\nu^{\prime}, \eta^{\prime}} \stackrel{M\left(\mathcal{O}_{x}\right)}{\times}\left(\operatorname{Bun}_{P} \underset{\operatorname{Bun}_{M}}{\times}{ }_{x} \mathcal{M}\right) \\
{ }^{\prime} h_{M}^{\leftarrow} \downarrow \\
\widetilde{\operatorname{id} \times p r^{\prime}} \downarrow \\
\widetilde{\operatorname{Bun}_{P}} \stackrel{\sim}{\longrightarrow} \operatorname{Gr}_{M}^{\nu^{\prime}} \stackrel{M\left(\mathcal{O}_{x}\right)}{\times}\left(\operatorname{Bun}_{P} \underset{\operatorname{Bun}_{M}}{\times}{ }_{x} \mathcal{M}\right),
\end{gathered}
$$

where ${ }_{x} \mathcal{M}$ denotes the canonical $M\left(\mathcal{O}_{x}\right)$-torsor over $\operatorname{Bun}_{M}$. Similarly, we have the identifications

$$
\begin{aligned}
& Z_{P, M}^{?, \eta, \eta^{\prime}} \stackrel{\sim}{\longrightarrow} \operatorname{Conv}_{M}^{\eta,-\mathrm{w}_{0}^{M}\left(\eta^{\prime}\right)} \stackrel{M\left(\mathcal{O}_{x}\right)}{\times}\left(\operatorname{Bun}_{P} \underset{\operatorname{Bun}_{M}}{\times}{ }_{x} \mathcal{M}\right) \\
& ' h_{\vec{M}} \downarrow \quad \text { id } \times p r^{\prime} \downarrow \\
& \widetilde{\operatorname{Bun}}_{P} \stackrel{\sim}{\longrightarrow} \operatorname{Gr}_{M}^{\eta} \stackrel{M\left(\mathcal{O}_{x}\right)}{\times}\left(\operatorname{Bun}_{P} \underset{\operatorname{Bun}_{M}}{\times}{ }_{x} \mathcal{M}\right) .
\end{aligned}
$$

Lemma 4.2.6. Under the above identifications, the stack $Z_{P, M}^{\nu^{\prime}, \eta, \eta^{\prime}}$ admits the following description:

(a) Inside $Z_{P, M}^{\nu^{\prime}, ?, \eta^{\prime}}$, it is identified with $\operatorname{Conv}_{M}^{\nu^{\prime}, \eta^{\prime} ; \eta} \stackrel{M\left(\mathcal{O}_{x}\right)}{\times}\left(\operatorname{Bun}_{P} \underset{\operatorname{Bun}_{M}}{\times}{ }_{x} \mathcal{M}\right)$.

(b) Inside $Z_{P, M}^{?, \eta, \eta^{\prime}}$, it is identified with $\operatorname{Conv}_{M}^{\eta,-w_{0}^{M}\left(\eta^{\prime}\right) ; \nu^{\prime}} \stackrel{M\left(\mathcal{O}_{x}\right)}{\times}\left(\operatorname{Bun}_{P} \underset{\operatorname{Bun}_{M}}{\times}{ }_{x} \mathcal{M}\right)$.

Let us consider the $*$-restriction of $\left(\mathcal{A}_{M}^{-\mathrm{w}_{0}^{M}(\nu)} \widetilde{\otimes} \mathrm{IC}_{\widetilde{\mathrm{Bun}_{P}}}\right)^{r}$ to $Z_{P, M}^{?, \eta, \eta^{\prime}}$. By definition, this complex lives in cohomological degrees $\leq 0$ (and the inequality is strict unless $\eta^{\prime}=\nu$ and $\left.\eta=0\right)$. Moreover, it is constant along the fibers of the projection

$$
Z_{P, M}^{?, \eta, \eta^{\prime}} \rightarrow{ }_{x, \eta} \widetilde{\operatorname{Bun}}_{P}
$$

Therefore, from the description of $Z_{P, M}^{\nu^{\prime}, \eta, \eta^{\prime}}$ given in Lemma 4.2.6(b), we infer that when we restrict this complex further to $Z_{P, M}^{\nu^{\prime}, \eta, \eta^{\prime}}$ it will live in cohomological degrees

$$
\leq-\operatorname{codim}\left(\operatorname{Conv}_{M}^{\eta,-\mathrm{w}_{0}^{M}\left(\eta^{\prime}\right) ; \nu^{\prime}}, \operatorname{Conv}_{M}^{\eta,-\mathrm{w}_{0}^{M}\left(\eta^{\prime}\right)}\right)=-\left\langle\eta+\eta^{\prime}-\nu^{\prime}, \check{\rho}_{M}\right\rangle,
$$

where the last inequality follows from (3). Moreover, the inequality is strict unless $\eta=0$ and $\eta^{\prime}=\nu$.

Let us observe now that according to Lemma 4.2.6.(a), the fibers of the map

$$
Z_{P, M}^{\nu^{\prime}, \eta, \eta^{\prime}} \rightarrow{ }_{x, \nu^{\prime}} \widetilde{\operatorname{Bun}}_{P}
$$

can be identified with the fibers of the projection

$$
p r^{\prime}: \operatorname{Conv}_{M}^{\nu^{\prime}, \eta^{\prime} ; \eta} \rightarrow \operatorname{Gr}_{M}^{\nu^{\prime}} .
$$

By applying again (3) we conclude that those have dimension $\leq\left\langle\eta+\eta^{\prime}-\nu^{\prime}, \check{\rho}_{M}\right\rangle$. 
This implies that when $\eta \neq 0$ or $\eta^{\prime} \neq \nu$ the complex $K_{M}^{\nu^{\prime}, \eta, \eta^{\prime}}$ lives in negative cohomological degrees and it remains to analyze the case $\eta=0, \eta^{\prime}=\nu$.

However, in the latter case $Z_{P, M}^{?, 0, \nu}$ coincides with $Z_{P, M}^{-\mathrm{w}_{0}^{M}(\nu), 0, \nu}$ and its projection onto ${ }_{x, \nu} \widetilde{\operatorname{Bun}}_{P}$ is an isomorphism. This implies the last statement of the proposition, since the $*$-restriction of $\left(\mathcal{A}_{M}^{-\mathrm{w}_{0}^{M}(\nu)} \widetilde{\otimes} \mathrm{IC}_{\widetilde{\mathrm{Bun}}_{P}}\right)^{r}$ to $Z_{P, M}^{?, 0, \nu}$ obviously coincides with $\mathrm{IC}_{Z_{P, M}^{?, 0, \nu}}$.

\subsection{Proof of Theorem 4.1.5.}

4.3.1. For the proof of Theorem 4.1.5 we need to recall several additional facts about affine Grassmannians.

Recall the lattice $\Lambda_{G, P}$ (cf. Sect. 1.3.1), which by definition can be identified with the lattice of characters of the torus $Z(\check{M})$. Let us observe, that $\Lambda_{G, P}$ can be identified, in addition, with the set of connected components of the affine Grassmannians $\operatorname{Gr}_{M /[M, M]}$ and $\mathrm{Gr}_{M}$.

For $\theta \in \Lambda_{G, P}$ consider the closed sub-scheme $\bar{S}_{P}^{\theta}$ of $\mathrm{Gr}_{G}$ that corresponds to those pairs $\left(\mathcal{F}_{G}, \beta:\left.\left.\mathcal{F}_{G}^{0}\right|_{\mathcal{D}_{x}^{*}} \rightarrow \mathcal{F}_{G}\right|_{\mathcal{D}_{x}^{*}}\right)$, for which the meromorphic map

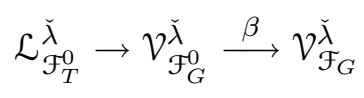

has a pole of order $\leq\langle\theta, \check{\lambda}\rangle$ for every $\check{\lambda} \in \check{\Lambda}_{G, P}$.

Similarly, let $S_{P}^{\theta}$ be an open subscheme of $\bar{S}_{P}^{\theta}$ that corresponds to the pairs $\left(\mathcal{F}_{G}, \beta\right)$ as above for which the maps

$$
\mathcal{L}_{\mathcal{F}_{T}^{0}}^{\check{\lambda}}(-\langle\theta, \check{\lambda}\rangle \cdot x) \rightarrow \mathcal{V}_{\mathcal{F}_{G}}^{\check{\lambda}}, \check{\lambda} \in \check{\Lambda}_{G, P}
$$

have no zero either.

The subscheme $S_{P}^{\theta}$ is stable under the action of the group $[P, P]\left(\mathcal{K}_{x}\right) M\left(\mathcal{O}_{x}\right)$ on $\mathrm{Gr}_{G}$. Moreover, the action of an even smaller group, namely, of $[P, P]\left(\mathcal{K}_{x}\right)$ on $S_{P}^{\theta}$ is transitive.

The following assertion is well-known:

Proposition 4.3.2. For each $\theta \in \Lambda_{G, P}$ there exists a unique map of indschemes

$$
\mathfrak{t}_{P}^{\theta}: S_{P}^{\theta} \rightarrow \operatorname{Gr}_{M}
$$

with the following property:

For $\left(\mathcal{F}_{G}, \beta:\left.\left.\mathcal{F}_{G}^{0}\right|_{\mathcal{D}_{x}^{*}} \rightarrow \mathcal{F}_{G}\right|_{\mathcal{D}_{x}^{*}}\right) \in S_{P}^{\theta}$, its image $\left(\mathcal{F}_{M}, \beta_{M}\right)$ is the unique point of $\mathrm{Gr}_{M}$, for which the composition

$$
\left(\mathcal{V}^{U(P)}\right)_{\mathcal{F}_{M}} \stackrel{\beta_{M}}{\longrightarrow}\left(\mathcal{V}^{U(P)}\right)_{\mathcal{F}_{M}^{0}} \rightarrow \mathcal{V}_{\mathcal{F}_{G}^{0}} \stackrel{\beta}{\longrightarrow} \mathcal{V}_{\mathcal{F}_{G}}
$$

has neither zero nor pole $\forall \check{\lambda} \in \check{\Lambda}_{G}^{+}$.

By definition, $\mathfrak{t}_{P}^{\theta}$ takes values in the connected component of $\operatorname{Gr}_{M}$ corresponding to $\theta$. Let $\nu$ be an element of $\Lambda_{M}^{+}$and let $\theta$ be its image under the projection $\Lambda \rightarrow \Lambda_{G, P}$. In what follows, we will denote by $S_{P}^{\nu}$ the pre-image $\mathfrak{t}_{P}^{\theta-1}\left(\mathrm{Gr}_{M}^{\nu}\right) \subset S_{P}^{\theta}$. We will denote by $\mathfrak{t}_{P}^{\nu}$ the corresponding map $S_{P}^{\nu} \rightarrow \mathrm{Gr}_{M}^{\nu}$.

The next result is a consequence of Proposition 3.2.6: 
Proposition 4.3.3. (a) Let $\nu$ (resp., $\lambda$ ) be a dominant integral coweight of $M$ (resp., of $G$ ) The intersection $S_{P}^{\nu} \cap \mathrm{Gr}_{G}^{\lambda}$ has dimension $\leq\langle\nu+\lambda, \check{\rho}\rangle$ and the fibers of the projection

$$
\mathfrak{t}_{P}^{\nu}: S_{P}^{\nu} \cap \mathrm{Gr}_{G}^{\lambda} \rightarrow \mathrm{Gr}_{M}^{\nu}
$$

are of dimension $\leq\langle\nu+\lambda, \check{\rho}\rangle-\left\langle\nu, 2 \check{\rho}_{M}\right\rangle$.

(b) For $\lambda \in \Lambda_{G}^{+}$and $\theta \in \Lambda_{G, P}$, the direct image

$$
\mathfrak{t}_{P !}^{\theta}\left(\left.\mathcal{A}_{G}^{\lambda}\right|_{S_{P}^{\theta}}\right) \otimes\left(\overline{\mathbb{Q}_{\ell}}[1]\left(\frac{1}{2}\right)\right)^{\otimes\left\langle\theta, 2\left(\check{\rho}-\check{\rho}_{M}\right)\right\rangle}
$$

lives in cohomological degrees $\leq 0$. (In the above formula we have used the fact that $2\left(\check{\rho}-\check{\rho}_{M}\right) \in \check{\Lambda}_{G, P}$.) Its 0 -th cohomology belongs to $\mathrm{Sph}_{M}$.

(c) The functor $\mathrm{Sph}_{G} \rightarrow \mathrm{Sph}_{M}$ given by

$$
\mathcal{S} \rightarrow \underset{\theta}{\oplus} h^{0}\left(\mathfrak{t}_{P !}^{\theta}\left(\left.\mathcal{S}\right|_{S_{P}^{\theta}}\right) \otimes\left(\overline{\mathbb{Q}_{\ell}}[1]\left(\frac{1}{2}\right)\right)^{\otimes\left\langle\theta, 2\left(\check{\rho}-\check{\rho}_{M}\right)\right\rangle}\right)
$$

has a natural structure of a tensor functor.

Finally, we will use the following theorem, which follows from Theorem 3.2 .8 (cf. [3]):

Theorem 4.3.4. The tensor functor

$$
\mathcal{S} \rightarrow \underset{\theta}{\oplus} h^{0}\left(\mathfrak{t}_{P !}^{\theta}\left(\left.\mathcal{S}\right|_{S_{P}^{\theta}}\right) \otimes\left(\overline{\mathbb{Q}_{\ell}}[1]\left(\frac{1}{2}\right)\right)^{\otimes\left\langle\theta, 2\left(\check{\rho}-\check{\rho}_{M}\right)\right\rangle}\right)
$$

of Proposition 4.3.3 (c) is canonically isomorphic to the functor $\operatorname{gRes}_{M}^{G}$ that makes the following diagram of categories commutative:

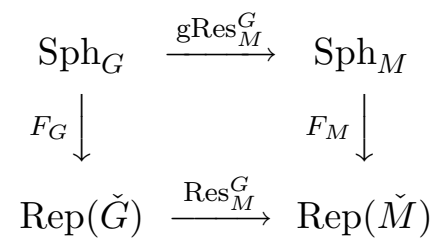

Corollary 4.3.5. The complex

$$
\mathfrak{t}_{P !}^{\nu !}\left(\left.\overline{\mathbb{Q}_{\ell}}\right|_{\operatorname{Gr}_{G}^{\lambda}}\right) \otimes\left(\overline{\mathbb{Q}_{\ell}}[1]\left(\frac{1}{2}\right)\right)^{\otimes\langle\lambda, 2 \check{\rho}\rangle+\left\langle\nu, 2\left(\check{\rho}-\check{\rho}_{M}\right)\right\rangle}
$$

lives in the cohomological degrees $\leq 0$ and its 0 -th perverse cohomology is canonically isomorphic to $\operatorname{IC}_{\mathrm{Gr}_{M}^{\nu}} \otimes \operatorname{Hom}_{\check{M}}\left(U^{\nu}, V^{\lambda}\right)$.

4.3.6. Now we will proceed to the proof of Theorem 4.1.5. The argument will be a direct generalization of the ones of Theorem 3.3.2 and Theorem 4.1.3.

As the complex ${ }_{x} H_{P, G}^{\lambda}\left(\mathrm{IC}_{\widetilde{\mathrm{Bun}}_{P}}\right)$ is self-dual, in order to prove that it is isomorphic to the direct sum $\underset{\nu \in \Lambda_{M}^{+}}{\oplus} \mathrm{IC}_{x, \geq \nu} \widetilde{\operatorname{Bun}}_{P} \otimes \operatorname{Hom}_{\check{M}}\left(U^{\nu}, V^{\lambda}\right)$, it is enough to show that the complex

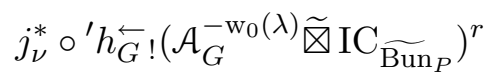


lives in non-positive cohomological degrees and that its 0-th cohomology can be identified with $\mathrm{IC}_{x, \nu} \widetilde{\operatorname{Bun}}_{P} \otimes \operatorname{Hom}_{\check{M}}\left(U^{\nu}, V^{\lambda}\right)$.

For $\nu, \nu^{\prime} \in \Lambda_{M}^{+}$and $\lambda^{\prime} \in \Lambda_{G}^{+}$, let $Z_{P, G}^{\nu, ?, \lambda^{\prime}}$ and $Z_{P, G}^{?, \nu^{\prime}, \lambda^{\prime}}$ denote the locally closed substacks of ${ }_{x, \infty} Z_{P, G}$ equal to

$$
{ }^{\prime} h_{G}^{\leftarrow}-1\left({ }_{x, \nu} \widetilde{\operatorname{Bun}}_{P}\right) \cap \overline{\mathfrak{p}}^{-1}\left({ }_{x} \mathcal{H}_{G}^{\lambda^{\prime}}\right) \text { and }{ }^{\prime} h_{G}{ }^{-1}\left({ }_{x, \nu^{\prime}} \widetilde{\operatorname{Bun}}_{P}\right) \cap^{\prime} \overline{\mathfrak{p}}^{-1}\left({ }_{x} \mathcal{H}_{G}^{\lambda^{\prime}}\right) \text {, respectively }
$$

Let ${ }_{x} \mathcal{P}$ denote the canonical $P\left(\mathcal{O}_{x}\right)$-torsor over ${ }_{x, 0} \widetilde{\operatorname{Bun}}_{P}$ (cf. Sect. 3.3.4) and let ${ }_{x} \stackrel{o}{\mathcal{P}}$ denote its restriction to the open substack $\operatorname{Bun}_{P} \subset{ }_{x, 0} \widetilde{\operatorname{Bun}}_{P}$.

We have the following identifications of stacks:

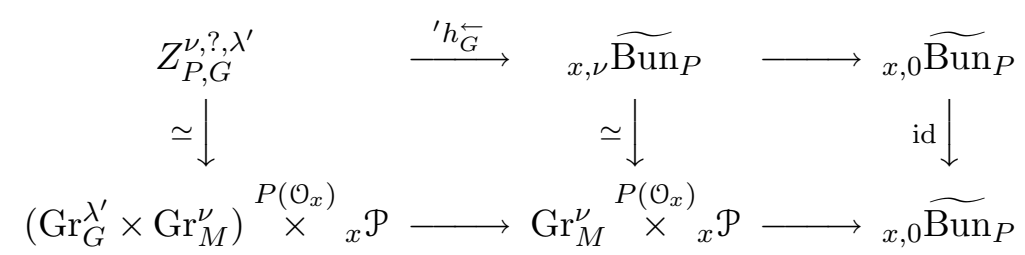

and

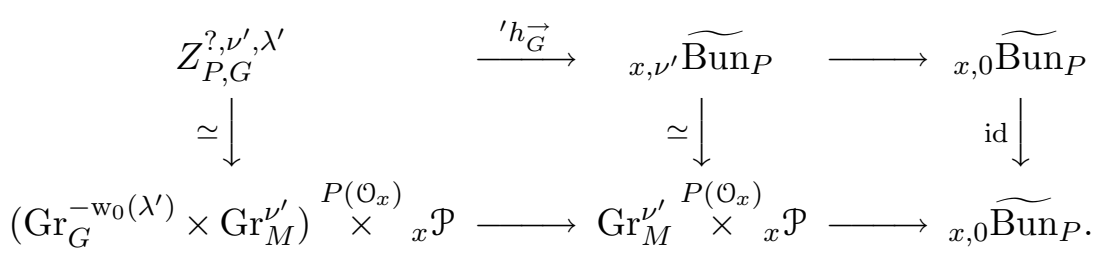

Now we need to introduce one more piece of notation. Let $\mu_{1}, \mu_{2}, \mu_{3}$ be elements of $\Lambda_{M}^{+}$and let $\xi$ be an element of $\Lambda_{G}^{+}$. Let us denote by $W^{\xi, \mu_{1}, \mu_{2} ; \mu_{3}}$ the following locally closed subscheme of $\mathrm{Gr}_{G} \times \mathrm{Gr}_{M}$ :

$W^{\xi, \mu_{1}, \mu_{2} ; \mu_{3}}$ consists of those quadruples

$$
\left(\mathcal{F}_{G}, \beta:\left.\left.\mathcal{F}_{G}\right|_{\mathcal{D}_{x}^{*}} \rightarrow \mathcal{F}_{G}^{0}\right|_{\mathcal{D}_{x}^{*}}, \mathcal{F}_{M}, \beta_{M}:\left.\left.\mathcal{F}_{M}\right|_{\mathcal{D}_{x}^{*}} \rightarrow \mathcal{F}_{M}^{0}\right|_{\mathcal{D}_{x}^{*}}\right)
$$

for which

- $\left(\mathcal{F}_{G}, \beta\right) \in S_{P}^{\mu_{1}} \cap \mathrm{Gr}_{G}^{\xi}$.

- $\left(\mathcal{F}_{M}, \beta_{M}\right) \in \mathrm{Gr}_{M}^{\mu_{2}}$.

- $\left(\left(\mathcal{F}_{M}, \beta_{M}\right) \times \mathfrak{t}_{P}^{\mu_{1}}\left(\mathcal{F}_{G}, \beta\right)\right) \in \operatorname{Gr}_{M}^{\mu_{2}} \times \operatorname{Gr}_{M}^{\mu_{1}}$ lies in the image of $\operatorname{Conv}_{M}^{\mu_{1}, \mu_{3} ; \mu_{2}}$ under the projection $p r \times p r^{\prime}: \operatorname{Conv}_{M} \rightarrow \mathrm{Gr}_{M} \times \mathrm{Gr}_{M}$.

Note that one can rephrase the third condition in the following way: the point $\left(\mathcal{F}_{M}, \beta_{M}\right) \in \mathrm{Gr}_{M}$ is in position $\mu_{3}$ with respect to $\mathfrak{t}_{P}^{\mu_{1}}\left(\mathcal{F}_{G}, \beta\right)$.

Finally, for $\nu, \nu^{\prime}, \eta \in \Lambda_{M}^{+}$and $\lambda^{\prime} \in \Lambda_{G}^{+}$we define the locally closed sub-stacks $Z_{P, G}^{\nu, \nu^{\prime}, \eta, \lambda^{\prime}}$ and $\widetilde{Z}_{P, G}^{\nu, \nu^{\prime}, \eta, \lambda^{\prime}}$ of ${ }_{x, \infty} Z_{P, G}$ as follows:

Using the identification of the first of the above commutative diagrams, $Z_{P, G}^{\nu, \nu^{\prime}, \eta, \lambda^{\prime}}$ is equal to

$$
W^{\lambda^{\prime}, \eta, \nu ; \nu^{\prime}} \stackrel{P\left(\mathcal{O}_{x}\right)}{\times}{ }_{x} \mathcal{P} \subset\left(\operatorname{Gr}_{G}^{\lambda^{\prime}} \times \mathrm{Gr}_{M}^{\nu}\right) \stackrel{P\left(\mathcal{O}_{x}\right)}{\times}{ }_{x} \mathcal{P}
$$


The stack $\widetilde{Z}_{P, G}^{\nu, \nu^{\prime}, \eta, \lambda^{\prime}}$ is defined via the second diagram as

$$
W^{-\mathrm{w}_{0}\left(\lambda^{\prime}\right),-\mathrm{w}_{0}^{M}(\eta), \nu^{\prime} ; \nu} \stackrel{P\left(\mathcal{O}_{x}\right)}{\times}{ }_{x} \mathcal{P} \subset\left(\mathrm{Gr}_{G}^{-\mathrm{w}_{0}\left(\lambda^{\prime}\right)} \times \operatorname{Gr}_{M}^{\nu^{\prime}}\right) \stackrel{P\left(\mathcal{O}_{x}\right)}{\times}{ }_{x} \mathcal{P} .
$$

Lemma 4.3.7. The sub-stacks $Z_{P, G}^{\nu, \nu^{\prime}, \eta, \lambda^{\prime}} \subset Z_{P, G}$ and $\widetilde{Z}_{P, G}^{\nu, \nu^{\prime}, \eta, \lambda^{\prime}} \subset Z_{P, G}$ coincide.

We define the complex $K^{\nu, \nu^{\prime}, \eta, \lambda^{\prime}}$ on ${ }_{x, \nu} \widetilde{\operatorname{Bun}}_{P}$ as the direct image under the map $h_{G}^{\overleftarrow{G}}: Z_{P, G}^{\nu, \nu^{\prime}, \eta, \lambda^{\prime}} \rightarrow{ }_{x, \nu} \widetilde{\operatorname{Bun}}_{P}$ of the $*$-restriction of $\left(\mathcal{A}_{G}^{-\mathrm{w}_{0}(\lambda)} \widetilde{\otimes} \mathrm{IC}_{\widetilde{\operatorname{Bun}_{P}}}\right)^{r}$ to $Z_{P, G}^{\nu, \nu^{\prime}, \eta, \lambda^{\prime}}$

4.3.8. To establish the required isomorphism, it is enough to show that

a) The complex $K^{\nu, \nu^{\prime}, \eta, \lambda^{\prime}}$ lives in cohomological degrees $\leq 0$ and the inequality is strict unless $\nu^{\prime}=0, \lambda^{\prime}=\lambda$ and $\eta=\nu$.

b) The $*$-restriction of $h^{0}\left(K^{\nu, 0, \nu, \lambda}\right)$ to ${ }_{x, \nu} \widetilde{\operatorname{Bun}}_{P}-{ }_{x, \nu} \operatorname{Bun}_{P}$ vanishes.

c) $h^{0}\left(K^{\nu, 0, \nu, \lambda}\right)$ restricted to ${ }_{x, \nu} \operatorname{Bun}_{P}$ is isomorphic to $\operatorname{IC}_{x, \nu} \operatorname{Bun}_{P} \otimes \operatorname{Hom}_{\check{M}}\left(U^{\nu}, V^{\lambda}\right)$.

First of all, as in the proofs of Theorem 3.3.2 and Theorem 4.1.3, the $*$-restriction of $\left(\mathcal{A}_{G}^{-\mathrm{w}_{0}(\lambda)} \widetilde{\nabla} \mathrm{IC}_{\widetilde{\mathrm{Bun}}_{P}}\right)^{r}$ to the substack $Z_{P, G}^{\nu, \nu^{\prime}, \eta, \lambda^{\prime}}=\widetilde{Z}_{P, G}^{\nu, \nu^{\prime}, \eta, \lambda^{\prime}}$ lives in cohomological degrees

$$
\leq-\operatorname{codim}\left(W^{-\mathrm{w}_{0}\left(\lambda^{\prime}\right),-\mathrm{w}_{0}^{M}(\eta), \nu^{\prime} ; \nu}, \operatorname{Gr}_{G}^{-\mathrm{w}_{0}\left(\lambda^{\prime}\right)} \times \operatorname{Gr}_{M}^{\nu^{\prime}}\right)
$$

and it follows from (3) and Proposition 4.3.3(a) that

$\operatorname{codim}\left(W^{-\mathrm{w}_{0}\left(\lambda^{\prime}\right),-\mathrm{w}_{0}^{M}(\eta), \nu^{\prime} ; \nu}, \operatorname{Gr}_{G}^{\lambda^{\prime}} \times \operatorname{Gr}_{M}^{\nu^{\prime}}\right)=\left\langle\lambda^{\prime}, \check{\rho}\right\rangle+\left\langle\mathrm{w}_{0}^{M}(\eta), \check{\rho}\right\rangle+\left\langle\nu^{\prime}-\mathrm{w}_{0}^{M}(\eta)-\nu, \check{\rho}_{M}\right\rangle$.

Now, the fibers of the map $h_{G}^{\overleftarrow{ }}: Z_{P, G}^{\nu, \nu^{\prime}, \eta, \lambda^{\prime}} \rightarrow{ }_{\nu, x} \widetilde{\operatorname{Bun}}_{P}$ have the same dimension as the fibers of the map $W^{\lambda^{\prime}, \eta, \nu ; \nu^{\prime}} \rightarrow \mathrm{Gr}_{M}^{\nu}$, and the latter equals $\left\langle\lambda^{\prime}, \check{\rho}\right\rangle+\langle\eta, \check{\rho}\rangle+\left\langle\nu^{\prime}-\nu-\eta, \check{\rho}_{M}\right\rangle$.

As $\left\langle\eta, \check{\rho}-\check{\rho}_{M}\right\rangle=\left\langle\mathrm{w}_{0}^{M}(\eta), \check{\rho}-\check{\rho}_{M}\right\rangle$, point (a) above follows. Point (b) follows in the same way as point (b) of Proposition 3.3.5.

Let us consider now the case $\lambda^{\prime}=\lambda, \nu^{\prime}=0$. (In this case $\eta$ is automatically equal to

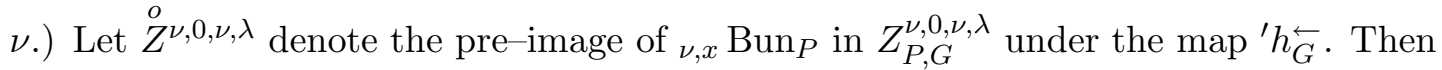
$\stackrel{o}{Z}^{\nu, 0, \nu, \lambda}$ is also the pre-image of $\operatorname{Bun}_{P}$ under the map ${ }^{\prime} h \vec{G}$.

We have the following commutative diagram of stacks:

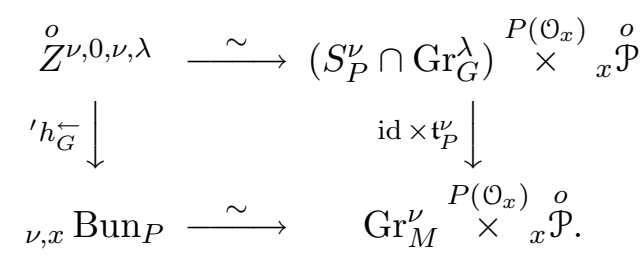

Moreover, as $\operatorname{Bun}_{P}$ is smooth, the restriction of $\left(\mathcal{A}_{G}^{-\mathrm{w}_{0}(\lambda)} \widetilde{\nabla} \mathrm{IC}_{\widetilde{\mathrm{Bun}_{P}}}\right)^{r}$ to $\stackrel{o}{Z}_{P, G}^{\nu, 0, \nu, \lambda}$ is the twisted external product

$$
\left(\overline{\mathbb{Q}_{\ell}}[1]\left(\frac{1}{2}\right)\right)_{S_{P}^{\nu} \cap \operatorname{Gr}_{G}^{\lambda}}^{\otimes\left\langle\lambda, \check{\rho} \breve{\rho}\left(2\left\langle\nu-\check{\rho}_{M}\right\rangle\right.\right.} \widetilde{\otimes} \mathrm{IC}_{\mathrm{Bun}_{P}} .
$$


Therefore, since the group $P\left(\mathcal{O}_{x}\right)$ is connected, Corollary 4.3.5 implies that the restriction of $h^{0}\left(K^{\nu, 0, \nu, \lambda}\right)$ to ${ }_{x, \nu} \operatorname{Bun}_{P}$ can be identified with $\mathrm{IC}_{x, \nu} \operatorname{Bun}_{P} \otimes \operatorname{Hom}_{\check{M}}\left(U^{\nu}, V^{\lambda}\right)$.

Hence, we have established the isomorphism

$$
{ }_{x} H_{P, G}^{\lambda}\left(\mathrm{IC}_{\widetilde{\mathrm{Bun}_{P}}}\right) \simeq \underset{\nu \in \Lambda_{M}^{+}}{\oplus} \mathrm{IC}_{x, \geq \nu} \widetilde{\operatorname{Bun}_{P}} \otimes \operatorname{Hom}_{\check{M}}\left(U^{\nu}, V^{\lambda}\right) .
$$

The fact that this isomorphism is compatible with the tensor structure, is a corollary of point (c) of Proposition 4.3 .3 combined with Theorem 4.3.4.

\section{THE ACYCLICITY THEOREM}

5.1. The notion of local acyclicity. Let $f: Y_{1} \rightarrow Y_{2}$ be a map between smooth algebraic varieties and let $\mathcal{S}$ be an object of $\operatorname{Sh}\left(Y_{1}\right)$. In [8], there was introduced the notion of universal local acyclicity (we will abbreviate it to ULA) of $\mathcal{S}$ with respect to $f$, which we are now going to review.

5.1.1. Let $g: Y^{\prime} \rightarrow Y^{\prime \prime}$ be a map between algebraic varieties. If $\mathcal{S}_{1}, \mathcal{S}_{2}$ are two objects of $\mathrm{Sh}\left(Y^{\prime \prime}\right)$, there exists a natural morphism in $\mathrm{Sh}\left(Y^{\prime}\right)$ :

$$
g^{*}\left(\underline{\operatorname{Hom}}\left(\mathcal{S}_{1}, \mathcal{S}_{2}\right)\right) \rightarrow \underline{\operatorname{Hom}}\left(g^{*}\left(\mathcal{S}_{1}\right), f^{*}\left(\mathcal{S}_{2}\right)\right),
$$

where $\underline{\text { Hom }}$ is the internal Hom.

Assume now that both $Y^{\prime}$ and $Y^{\prime \prime}$ are smooth and take

$$
\mathcal{S}_{2}:=\mathbb{D}_{Y^{\prime \prime}} \simeq\left(\overline{\mathbb{Q}_{\ell}}(1)[2]\right)^{\otimes \operatorname{dim}\left(Y^{\prime \prime}\right)} .
$$

Then (5) yields us a functorial map

$$
g^{*}(\mathbb{D}(\mathcal{S})) \otimes\left(\overline{\mathbb{Q}_{\ell}}(1)[2]\right)^{\otimes \operatorname{dim}\left(Y^{\prime}\right)-\operatorname{dim}\left(Y^{\prime \prime}\right)} \rightarrow \mathbb{D}\left(g^{*}(\mathcal{S})\right),
$$

and by replacing $\mathcal{S}$ by $\mathbb{D}(\mathcal{S})$ we obtain a functorial map

$$
\operatorname{can}_{g}: g^{*}(\mathcal{S}) \otimes\left(\overline{\mathbb{Q}_{\ell}}\left(\frac{1}{2}\right)[1]\right)^{\otimes \operatorname{dim}\left(Y^{\prime}\right)-\operatorname{dim}\left(Y^{\prime \prime}\right)} \rightarrow g^{!}(\mathcal{S}) \otimes\left(\overline{\mathbb{Q}_{\ell}}\left(\frac{1}{2}\right)[1]\right)^{\otimes \operatorname{dim}\left(Y^{\prime \prime}\right)-\operatorname{dim}\left(Y^{\prime}\right)} .
$$

The above natural transformation $\mathrm{can}_{g}$ is clearly an isomorphism when $g$ is smooth. Let us point out that in general, the map $\mathrm{can}_{g}$ is not expressible via the standard six functors. As an incarnation of this fact, it is rather hard to define it on the level of D-modules.

5.1.2. Now let $f: Y_{1} \rightarrow Y_{2}$ be a map between smooth algebraic varieties. Take $Y^{\prime}=Y_{1}, Y^{\prime \prime}=Y_{1} \times Y_{2}$ and $g=\Gamma_{f}$. According to the above discussion, for any $\mathcal{S} \in \operatorname{Sh}\left(Y_{1}\right)$ and $\mathcal{T} \in \operatorname{Sh}\left(Y_{2}\right)$, we obtain a canonical map

$$
\operatorname{can}_{\Gamma_{f}}: \mathcal{S} \otimes f^{*}(\mathcal{T}) \otimes\left(\overline{\mathbb{Q}_{\ell}}\left(\frac{1}{2}\right)[1]\right)^{\otimes-\operatorname{dim}\left(Y_{2}\right)} \rightarrow \mathcal{S} \stackrel{!}{\otimes} f^{!}(\mathcal{T}) \otimes\left(\overline{\mathbb{Q}_{\ell}}\left(\frac{1}{2}\right)[1]\right)^{\otimes \operatorname{dim}\left(Y_{2}\right)},
$$

where $\stackrel{!}{\otimes}$ is the $\mathbb{D}$-conjugate of $\otimes$, i.e. $\mathcal{S}_{1} \stackrel{!}{\otimes} \mathcal{S}_{2}:=\mathbb{D}\left(\mathbb{D}\left(\mathcal{S}_{1}\right) \otimes \mathbb{D}\left(\mathcal{S}_{2}\right)\right)$.

Definition. An object $\mathcal{S} \in \mathrm{Sh}\left(Y_{1}\right)$ is said to be locally acyclic with respect to $f$ if can $_{\Gamma_{f}}$ is an isomorphism $\forall \mathcal{T} \in \operatorname{Sh}\left(Y_{2}\right)$. A sheaf $\mathcal{S}$ is universally locally acyclic (ULA) with respect to $f$, if the above property holds after any smooth base change $Y_{2}^{\prime} \rightarrow Y_{2}$. 
For instance, any sheaf on $Y_{1}$ is ULA with respect to the projection $Y_{1} \rightarrow$ pt. When $f$ is the identity map $Y_{1} \rightarrow Y_{1}$, one can show that $\mathcal{S}$ is ULA if and only of it is locally constant.

Remark. The above definition of universal local acyclicity is a priori weaker than the one given in [8]. (In other words, if a complex $\mathcal{S}$ is ULA in the sense of loc. cit., then it is ULA in our sense as well.) We conjecture that the two notions are in fact equivalent.

However, for the purposes of this paper, it does not matter which definition to use: the proof of the main result, Theorem 5.1.5, works for both of them.

Here are the most immediate properties of the ULA condition:

1. Let $\mathcal{S}$ be an ULA sheaf on $Y_{1}$ with respect to $f: Y_{1} \rightarrow Y_{2}$. Let $Y^{\prime}$ be another smooth variety and $\mathcal{S}^{\prime}$ be an arbitrary sheaf on it. Then the sheaf $\mathcal{S} \otimes \mathcal{S}^{\prime}$ on $Y_{1} \times Y^{\prime}$ is ULA with respect to the map $Y_{1} \times Y^{\prime} \rightarrow Y_{1} \stackrel{f}{\rightarrow} Y_{2}$. (In most applications we will have $Y_{2}=Y_{1}, \mathcal{S}:=\overline{\mathbb{Q}}_{\ell Y_{1}}$.)

2. The ULA condition is local in the smooth topology on the source. In other words, if $s: Y_{1} \rightarrow Y_{1}^{\prime}$ is a smooth (resp., smooth and surjective) map and $\mathcal{S}$ is a sheaf on $Y_{1}^{\prime}$, then the sheaf $s^{*}(\mathcal{S})$ on $Y_{1}$ is ULA with respect to $f:=f^{\prime} \circ s$ if (resp., if and only if) the sheaf $\mathcal{S}$ on $Y_{1}^{\prime}$ is ULA with respect to $f^{\prime}$.

3. If $s: Y_{1} \rightarrow Y_{1}^{\prime}$ is a proper map (resp., closed embedding) and $\mathcal{S}$ is a sheaf on $Y_{1}$, then the sheaf $s_{*}(\mathcal{S})$ on $Y_{1}^{\prime}$ is ULA with respect to $f^{\prime}$ (we are assuming that $f$ factorizes as $f=f^{\prime} \circ s$ ) if (resp., if and only if) the sheaf $\mathcal{S}$ is ULA with respect to $f$.

This property allows to formulate the ULA condition in the situation when $Y_{1}$ is not necessarily smooth: it is enough to (locally) embed $Y_{1}$ as a closed sub-scheme into some $Y_{1}^{\prime}$ (with $f=f^{\prime} \circ s$ and $Y_{1}^{\prime}$ being smooth) and to require that the sheaf $s_{*}(\mathcal{S})$ on $Y_{1}^{\prime}$ is ULA with respect to $f^{\prime}$.

4. A sheaf $\mathcal{S}$ is ULA if and only $\mathbb{D}(\mathcal{S})$ is.

5. Let $\mathcal{S}$ is a ULA sheaf on $Y_{1}$ with respect to $f: Y_{1} \rightarrow Y_{2}$ and consider the functor $\operatorname{Sh}\left(Y_{2}\right) \rightarrow \operatorname{Sh}\left(Y_{1}\right)$ given by $\mathcal{T} \rightarrow \mathcal{S} \otimes f^{*}(\mathcal{T}) \otimes\left(\overline{\mathbb{Q}}\left(\frac{1}{2}\right)[1]\right)^{\otimes-\operatorname{dim}\left(Y_{2}\right)}$. Then it commutes with the Verdier duality in the sense that there is an isomorphism of functors:

$$
\mathbb{D}\left(\mathcal{S} \otimes f^{*}(\mathcal{T}) \otimes\left(\overline{\mathbb{Q}_{\ell}}\left(\frac{1}{2}\right)[1]\right)^{\otimes-\operatorname{dim}\left(Y_{2}\right)}\right) \simeq \mathbb{D}(\mathcal{S}) \otimes f^{*}(\mathbb{D}(\mathcal{T})) \otimes\left(\overline{\mathbb{Q}_{\ell}}\left(\frac{1}{2}\right)[1]\right)^{\otimes-\operatorname{dim}\left(Y_{2}\right)}
$$

Moreover, when $\mathcal{S}$ is concentrated in non-positive (resp., non-negative) cohomological degrees, the above functor is right (resp., left) exact.

6. If $t: Y_{2} \rightarrow Y_{2}^{\prime}$ is a smooth map and $\mathcal{S}$ is a sheaf on $Y_{1}$ which is ULA with respect to $f: Y_{1} \rightarrow Y_{2}$, then $\mathcal{S}$ is ULA with respect to $t \circ f$.

An important technical tool is provided by the following theorem (cf. [8]):

Theorem 5.1.3. Let $f: Y_{1} \rightarrow Y_{2}$ be a map, where $Y_{1}$ is a scheme of finite type and $Y_{2}$ is a smooth variety. Let $\mathcal{S}$ be an object of $\mathrm{Sh}\left(Y_{1}\right)$. Then there exists a non-empty open subvariety $Y_{1}^{0} \subset Y_{1}$, such that $\mathcal{S}$ is $U L A$ when restricted to $f^{-1}\left(Y_{1}^{0}\right) \subset Y_{2}$. 
5.1.4. The main result of this section is the following theorem:

Theorem 5.1.5. The sheaves $\mathrm{IC}_{\widetilde{\mathrm{Bun}}_{P}}$ and $\widetilde{j}_{P !}\left(\mathrm{IC}_{\mathrm{Bun}_{P}}\right)$ on $\widetilde{\mathrm{Bun}_{P}}$ are ULA with respect to the map $\widetilde{\mathfrak{q}}_{P}: \widetilde{\operatorname{Bun}}_{P} \rightarrow \operatorname{Bun}_{M}$.

The proof will be given in the next two subsections. However, we will present the proof only of the first part of Theorem 5.1.5, namely that $\mathrm{IC}_{\mathrm{Bun}_{P}}$ is ULA with respect to $\widetilde{\mathfrak{q}}_{P}$. The proof for $\widetilde{j}_{P !}\left(\mathrm{IC}_{\mathrm{Bun}}\right)$ is absolutely analogous.

Let us show how Theorem 5.1.5 implies Theorem 2.3.2 and, in particular, Theorem 2.1.2.

Proof. (of Theorem 2.3.2)

Point (a) of Theorem 2.3.2 follows immediately from Property 5 of Sect. 5.1.2. Moreover, by the same reason, for a perverse sheaf $\mathcal{T}$ on $\operatorname{Bun}_{M}$, the sheaf $\widetilde{\mathfrak{q}}_{P}^{! *}(\mathcal{T})$ is perverse.

Therefore, to prove point (b) of the Theorem, it is enough to show that whenever $\mathcal{T}$ is irreducible, $\widetilde{\mathfrak{q}}_{P}^{! *}(\mathcal{T})$ is irreducible as well. First of all, since the map $\mathfrak{q}: \operatorname{Bun}_{P} \rightarrow \operatorname{Bun}_{M}$ is smooth (and has connected fibers), it is clear that $\left.\mathfrak{q}_{P}^{! *}(\mathcal{T}) \simeq \widetilde{\mathfrak{q}}_{P}^{! *}(\mathcal{T})\right|_{\text {Bun }}$ is irreducible. Since the situation is Verdier self-dual, it is enough to show, therefore, that the $*-$ restriction of $\widetilde{\mathfrak{q}}_{P}^{! *}(\mathcal{T})$ to $\widetilde{\operatorname{Bun}}_{P}-\operatorname{Bun}_{P}$ lives in the cohomological degrees $<0$.

Let $K$ be the cone of the map $\widetilde{j}_{P !}\left(\mathrm{IC}_{\mathrm{Bun}_{P}}\right) \rightarrow \mathrm{IC}_{\widetilde{\mathrm{Bun}}_{P}}$. By definition, $K$ lives in the cohomological degress $<0$. 0 Moreover, Theorem 5.1.5 implies that the sheaf $K$ is also ULA with respect to $\widetilde{\mathfrak{q}}_{P}$.

However,

$$
\left.\widetilde{\mathfrak{q}}_{P}^{! *}(\mathcal{T})\right|_{\widetilde{\operatorname{Bun}}_{P}-\operatorname{Bun}_{P}} \simeq K \otimes \widetilde{\mathfrak{q}}_{P}^{*}(\mathcal{T}) \otimes\left(\overline{\mathbb{Q}}\left(\frac{1}{2}\right)[1]\right)^{\otimes-\operatorname{dim}\left(\operatorname{Bun}_{M}\right)}
$$

and the required assertion follows from the Property 5 of Sect. 5.1.2.

\subsection{Proof of Theorem 5.1.5 in the Borel case.}

\subsubsection{We will use the following observation:}

Let $H$ be a group (or a group-stack), let $Y \stackrel{f}{\longrightarrow} H$ be an arbitrary map and let $\mathcal{S}$ be a sheaf on $Y$. Consider the composition

$$
m_{f}: H \times Y \stackrel{f \times \text { id }}{\longrightarrow} H \times H \stackrel{\text { mult }}{\longrightarrow} H .
$$

We claim that the sheaf $\overline{\mathbb{Q}}_{\ell} \otimes \mathcal{S}$ is always ULA with respect to $m_{f}$. Indeed, the automorphism $(h, y) \mapsto(h \cdot f(y), y)$ transforms the data of $\left(m_{f}, \overline{\mathbb{Q}}_{\ell} \otimes \mathcal{S}\right)$ into a direct product situation (cf. Property 1 of Sect. 5.1.2).

The proof of Theorem 5.1.5 will be essentially a reduction of our situation $(\overline{\mathfrak{q}}$ : $\left.\overline{\operatorname{Bun}}_{B} \rightarrow \operatorname{Bun}_{T}\right)$ to the one mentioned above, where the role of $H$ will be played by $\operatorname{Bun}_{T}$. Namely, we will construct a stack $Z$ that fits into a commutative diagram:

\footnotetext{
${ }^{5}$ The last assertion uses, of course, Proposition 1.3.7, which will be independently proven later (cf. Sect. 6.1 and Sect. 6.2.).
} 


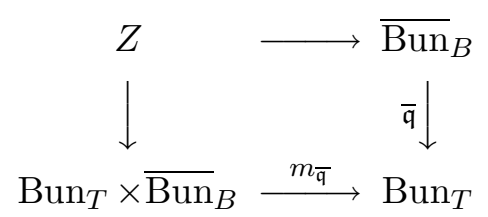

where the upper horizontal arrow is surjective and smooth and the left vertical arrow is just smooth. This will prove Theorem 5.1.5 in view of Property 2 of Sect. 5.1.2.

5.2.2. Let us choose elements $\lambda_{1}, \ldots, \lambda_{r} \in \Lambda_{G}^{+}$in such a way that they form a basis for $\Lambda \underset{\mathbb{Z}}{\otimes} \mathbb{Q}$. Let $m$ be an integer $\geq 2 g-1$ and consider the product $X^{m \cdot r}-\Delta$, where $\Delta$ denotes the divisor of diagonals.

Let $\mathcal{H}_{G}^{?}$ denote the following version of the Hecke stack: $\mathcal{H}_{G}^{?}$ classifies the data of

$$
\left(\left\{x_{1,1}, \ldots, x_{1, m}, x_{2,1}, \ldots, x_{r, 1}, \ldots, x_{r, m}\right\} \in X^{m \cdot r}-\Delta, \mathcal{F}_{G}, \mathcal{F}_{G}^{\prime}, \beta\right),
$$

where $\beta$ is an isomorphism

$$
\beta:\left.\mathcal{F}_{G} \simeq \mathcal{F}_{G}^{\prime}\right|_{X-\left\{x_{1,1}, \ldots, x_{1, m}, x_{2,1}, \ldots, x_{r, 1}, \ldots, x_{r, m}\right\}}
$$

such that for every $i$ and $j, \mathcal{F}_{G}$ is in position $\lambda_{i}$ with respect to $\mathcal{F}_{G}^{\prime}$ at $x_{i, j}$.

We let $h_{G}^{\leftarrow}$ and $h_{G}$ denote the projections from $\mathcal{H}_{G}^{?}$ to $\operatorname{Bun}_{G}$ that send the above point of $\mathcal{H}_{G}^{?}$ to $\mathcal{F}_{G}$ and $\mathcal{F}_{G}^{\prime}$, respectively. The projection from $\mathcal{H}_{G}^{?} \rightarrow X^{m \cdot r}-\Delta$ will be denoted by $\pi$.

Let us denote by $\bar{Z}$ the fiber product $\mathcal{H}_{G}^{\text {? }} \underset{\operatorname{Bun}_{G}}{\times} \overline{\operatorname{Bun}}_{B}$, where $\mathcal{H}_{G}$ is mapped to $\operatorname{Bun}_{G}$ by means of $h_{G}$. As in Sect. 3.1.1, we have the second projection $\phi: \bar{Z} \rightarrow \overline{\operatorname{Bun}}_{B} \times$ $\left(X^{m \cdot r}-\Delta\right)$ and we obtain a commutative diagram:

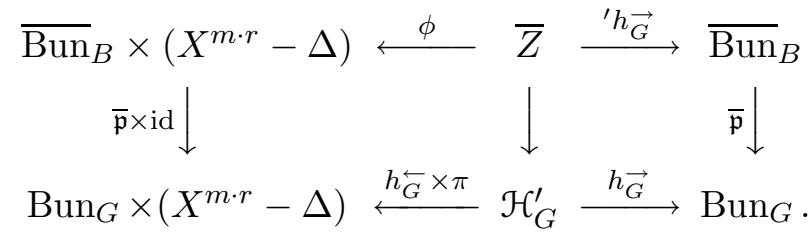

In addition, we have the Abel-Jacobi map AJ : $\left(X^{m \cdot r}-\Delta\right) \rightarrow \operatorname{Bun}_{T}$ that maps

$$
\left(x_{1,1}, \ldots, x_{1, m}, x_{2,1}, \ldots, x_{r, 1}, \ldots, x_{r, m}\right) \rightarrow \mathcal{F}_{T}^{0}\left(\sum_{i, j} \lambda_{i} \cdot x_{i, j}\right)
$$

and the map $\bar{Z} \stackrel{{ }^{\prime} h_{\vec{G}}}{\longrightarrow} \overline{\operatorname{Bun}}_{B} \stackrel{\overline{\mathfrak{q}}}{\longrightarrow} \operatorname{Bun}_{T}$ coincides with the composition

$$
\bar{Z} \stackrel{\phi}{\longrightarrow} \overline{\operatorname{Bun}}_{B} \times\left(X^{m \cdot r}-\Delta\right) \stackrel{\text { id } \times \mathrm{AJ}}{\longrightarrow} \overline{\operatorname{Bun}}_{B} \times \operatorname{Bun}_{T} \stackrel{m_{\bar{q}}}{\longrightarrow} \operatorname{Bun}_{T} .
$$

The sought-for stack $Z$ is defined as an open sub-stack of $\bar{Z}$ :

A point $\left(\left\{x_{i, j}\right\}, \mathcal{F}_{G}, \beta, \mathcal{F}_{G}^{\prime}, \mathcal{F}_{T}^{\prime},\left\{\kappa^{\prime \check{\lambda}}: \mathcal{L}_{\mathcal{F}_{T}^{\prime}}^{\check{L}} \hookrightarrow \mathcal{V}_{\mathcal{F}_{G}^{\prime}}^{\check{\lambda}}\right\}\right)$ belongs to $Z$ if the following holds:

a) The map $\kappa^{\prime \check{\lambda}}: \mathcal{L}_{\mathcal{F}_{T}^{\prime}}^{\check{L}} \hookrightarrow \mathcal{V}_{\mathcal{F}_{G}^{\prime}}^{\check{\lambda}}$ has no zero at any of the points $x_{i, j}, \forall \check{\lambda} \in \check{\Lambda}_{G}^{+}$.

b) The map $\kappa^{\check{\lambda}}:=\mathcal{L}_{\mathcal{F}_{T}^{\prime}}^{\check{\check{\nu}}}\left(-\sum_{i, j}\left\langle\lambda_{i}, \check{\lambda}\right\rangle \cdot x_{i, j}\right) \hookrightarrow \mathcal{V}_{\mathcal{F}_{G}}^{\check{\check{\lambda}}}$ has no zero at any of the points $x_{i, j}$ either, $\forall \check{\lambda} \in \check{\Lambda}_{G}^{+}$. 
It remains, therefore, to check that $Z$ defined in the above way satisfies all the requirements.

The projection ${ }^{\prime} h \vec{G}: \bar{Z} \rightarrow \overline{\mathrm{Bun}}_{B}$ is smooth by definition, hence, so is the map $Z \rightarrow \overline{\operatorname{Bun}}_{B}$. The fact that this map is surjective follows from Lemma 3.3.6(b).

Now, the restriction of $\phi$ onto $Z$ is an isomorphism, as follows from Lemma 3.3.6(c), and the map AJ : $\left(X^{m \cdot r}-\Delta\right) \rightarrow \operatorname{Bun}_{T}$ is smooth due to the condition that $m \geq 2 g-1$. This finishes the proof of Theorem 5.1.5 when $P=B$.

5.3. Proof of Theorem 5.1.5 in the general case. In principle, it is not difficult to generalize the proof given in the previous subsection to treat the case of an arbitrary parabolic $P$. However, we will present here a different argument, based on Theorem 5.1.3.

5.3.1. As a first step, we will exhaust the stack $\widetilde{\operatorname{Bun}}_{P}$ by open substacks which would be of finite type over $\operatorname{Bun}_{M}$. This can be done as follows:

Consider the stack $\overline{\operatorname{Bun}}_{P}$ and for an element $\theta \in \Lambda_{G, P}^{\text {pos }}$ consider the open substack $\overline{\operatorname{Bun}}_{P} \leq \theta \subset \overline{\operatorname{Bun}}_{P}$ that corresponds to parabolic Drinfeld's structures

$$
\left(\mathcal{F}_{G}, \mathcal{L}_{\mathcal{F}_{M /[M, M]}^{\check{\nu}}}^{\check{2}} \hookrightarrow \mathcal{V}_{\mathcal{F}_{G}}^{\check{\lambda}}, \check{\lambda} \in \check{\Lambda}_{G, P} \cap \check{\Lambda}_{G}^{+}\right)
$$

whose "total singularity" does not exceed $\theta$. This means that for every $\check{\lambda} \in \check{\Lambda}_{G, P} \cap \check{\Lambda}_{G}^{+}$, the coherent sheaf $\mathcal{V}_{\mathcal{F}_{G}}^{\check{\nearrow}} / \mathcal{L}_{\mathcal{F}_{M /[M, M]}}^{\check{\lambda}}$ has no torsion subsheaves of length $>\langle\theta, \check{\lambda}\rangle$.

Let $\widetilde{\operatorname{Bun}}_{P}^{\leq \theta} \subset \widetilde{\operatorname{Bun}}_{P}$ denote the pre-image of $\overline{\operatorname{Bun}}_{P}^{\leq \theta} \subset \overline{\operatorname{Bun}}_{P}$ under the map $\mathfrak{r}_{P}$. For instance, when $\theta=0, \widetilde{\operatorname{Bun}_{P}} \leq 0$ coincides with $\operatorname{Bun}_{P}$.

Lemma 5.3.2. For every $\theta$, the stack $\widetilde{\operatorname{Bun}_{P}} \leq \theta$ is of finite type over $\operatorname{Bun}_{M}$.

It is clear that $\widetilde{\operatorname{Bun}}_{P}=\cup_{\theta} \widetilde{\operatorname{Bun}}_{P}^{\leq \theta}$ and as the assertion of Theorem 5.1.5 is local on $\widetilde{\operatorname{Bun}}_{P}$, it would be sufficient to prove that $\forall \theta \in \Lambda_{G, P}^{\text {pos }}, \mathrm{IC}_{\widetilde{\operatorname{Bun}}} \leq \theta$ is locally acyclic with respect to $\widetilde{\mathfrak{q}}_{P}$. Therefore, in order not to overload the notation, we will now fix some $\theta$ and until the end of this section, we will replace the notation " $\widetilde{\operatorname{Bun}} \leq \theta$ " simply by " $\widetilde{\operatorname{Bun}}_{P}$ ". The reader will readily check that this open substack is stable under all the manipulations that we are about to perform.

5.3.3. Let $\stackrel{o}{\mathrm{Bun}_{M}}$ denote the maximal open substack of $\mathrm{Bun}_{M}$, over which $\mathrm{IC}_{\widetilde{\mathrm{Bun}} P}$ is ULA with respect to $\widetilde{\mathfrak{q}}_{P}$. Due to the finite type property above (Lemma 5.3.2) and Theorem 5.1.3, $\stackrel{\circ}{B}_{M}$ is non-empty.

Let $\mathcal{F}_{M}$ and $\mathcal{F}_{M}^{\prime}$ be two $\overline{\mathbb{F}_{q}}$-points of $\operatorname{Bun}_{M}$. We will write that $\mathcal{F}_{M} \prec \mathcal{F}_{M}^{\prime}$ if the following condition holds:

There exists a $G$-dominant coweight $\lambda \in \Lambda_{M}^{+}$such that the pair $\left(\mathcal{F}_{M}, \mathcal{F}_{M}^{\prime}\right)$ is the image under the map

$$
\mathcal{H}_{M}^{\lambda} \stackrel{h_{M}^{\leftarrow} \times h_{M}}{\longrightarrow} \operatorname{Bun}_{M} \times \operatorname{Bun}_{M}
$$


of some $\overline{\mathbb{F}_{q}}$-point of $\mathcal{H}_{M}^{\lambda}$.

Let $\sim$ be the equivalence relation on the set of isomorphism classes of $\overline{F_{q}}$-points of $\operatorname{Bun}_{M}$, generated by $\prec$. Theorem 5.1.5 clearly follows from the next two assertions:

Proposition 5.3.4. If $\mathcal{F}_{M} \prec \mathcal{F}_{M}^{\prime}$ then $\mathcal{F}_{M}^{\prime} \in{\stackrel{o}{\operatorname{Bun}_{M}}}_{M}$ if and only if $\mathcal{F}_{M}$ belongs to Bun $_{M}$ too.

Proposition 5.3.5. All $\overline{\mathbb{F}_{q}}$-points of $\operatorname{Bun}_{M}$ are $\sim$-equivalent.

We will first prove Proposition 5.3.5.

Proof. The assertion of the proposition is in fact an easy corollary of the following fact proven in [10]:

Lemma 5.3.6. Let $\widetilde{M}$ be a semi-simple group and let $\mathcal{F}_{\widetilde{M}}$ and $\mathcal{F}_{\widetilde{M}}^{\prime}$ be two $\widetilde{M}$-bundles on $\bar{X}$. Then for any $x \in X\left(\overline{\mathbb{F}_{q}}\right)$, the restrictions $\left.\mathcal{F}_{\widetilde{M}}\right|_{\bar{X}-x}$ and $\left.\mathcal{F}_{\widetilde{M}}^{\prime}\right|_{\bar{X}-x}$ become isomorphic.

Let $\mathcal{F}_{M}$ and $\mathcal{F}_{M}^{\prime}$ be two $\overline{\mathbb{F}_{q}}$-points of $\operatorname{Bun}_{M}$ and let $\mathcal{F}_{\widetilde{M}}$ and $\mathcal{F}_{\widetilde{M}}^{\prime}$ denote their reductions to $\widetilde{M}:=M / Z^{0}(M)$, where $Z^{0}(M)$ is the connected component of the identity of $Z(M)$.

The map $\Lambda_{G}^{+} \rightarrow \Lambda_{\widetilde{M}}^{+}$is surjective, therefore one can find $\lambda \in \Lambda_{G}^{+}$and a point of $\mathcal{H}_{M}^{\lambda}$ which projects to a point $\left(\mathcal{F}_{M}, \mathcal{F}_{M}^{\prime \prime}\right) \in \operatorname{Bun}_{M} \times \operatorname{Bun}_{M}$, where $\mathcal{F}_{M}^{\prime}$ and $\mathcal{F}_{M}^{\prime \prime}$ have the same reduction to $\widetilde{M}$.

We have: $\mathcal{F}_{M}^{\prime \prime}=\mathcal{F}_{M}^{\prime} \otimes \mathcal{F}_{Z^{0}(M)}$, where $\mathcal{F}_{Z^{0}(M)}$ is some $Z^{0}(M)$-bundle. Now the proof follows from the fact that $\Lambda_{G}^{+} \cap \Lambda_{Z^{0}(M)}$ spans $\Lambda_{Z^{0}(M)} \underset{\mathbb{Z}}{\otimes} \mathbb{Q}$.

\section{3 .7 .}

Proof. (of Proposition 5.3.4.)

Let us first prove the "if" part of the proposition. We will fix $\lambda \in \Lambda_{G}^{+}$and let us denote by $\bar{Z}$ the fiber product $\mathcal{H}_{G}^{\lambda} \underset{\operatorname{Bun}_{G}}{\times} \widetilde{\operatorname{Bun}_{P}}$, where $\mathcal{H}_{G}^{\lambda}$ is mapped to Bun ${ }_{G}$ by means of the projection $h_{\vec{G}}$.

Let also rel, $\widetilde{\operatorname{Bun}}_{P} \rightarrow X$ (resp., rel, $\geq \nu \widetilde{\operatorname{Bun}}_{P}, r_{r e l, \nu} \widetilde{\operatorname{Bun}}_{P}$ ) denote the relative version of the stack ${ }_{x, \infty} \widetilde{\operatorname{Bun}}_{P}$ (resp., $x_{x, \geq \nu} \widetilde{\operatorname{Bun}}_{P},{ }_{x, \nu} \widetilde{\operatorname{Bun}}_{P}$ ) introduced in Sect. 4.1.1. In other words, the fiber of $r e l, \infty \widetilde{\operatorname{Bun}}_{P}$ over $x \in X$ is ${ }_{x, \infty} \widetilde{\operatorname{Bun}}_{P}$ and similarly for ${ }_{r e l, \geq \nu} \widetilde{\operatorname{Bun}}_{P}$ and $r e l, \nu \widetilde{\operatorname{Bun}}_{P}$.

As in Sect. 4.1.4, there exists a second projection ${ }^{\prime} h_{G}^{\overleftarrow{Z}}: \bar{Z} \rightarrow{ }_{r e l, \infty} \widetilde{\operatorname{Bun}}_{P}$ :

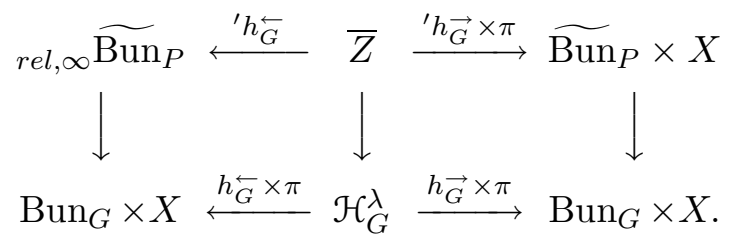


Let $Z \subset \bar{Z}$ be the following locally closed substack:

$$
Z:={ }^{\prime} h_{G}^{-1}\left(_{r e l, \lambda} \widetilde{\operatorname{Bun}}_{P}\right) \cap^{\prime} h_{G}^{-1}\left({ }_{r e l, 0} \widetilde{\operatorname{Bun}}_{P}\right)
$$

$\left(\right.$ rel, $0 \widetilde{\operatorname{Bun}}_{P}$ is, according to our conventions, an open substack of $\widetilde{\operatorname{Bun}}_{P} \times X$.)

Thus, $Z$ classifies the data of

$$
z=\left(x, \mathcal{F}_{G}, \mathcal{F}_{M}, \widetilde{\kappa}_{P}, \mathcal{F}_{G}^{\prime}, \mathcal{F}_{M}^{\prime}, \widetilde{\kappa}_{P}^{\prime}, \beta, \beta_{M}\right),
$$

where $\left(x, \mathcal{F}_{G}, \mathcal{F}_{M}, \widetilde{\kappa}_{P}\right) \in{ }_{x, 0} \widetilde{\operatorname{Bun}}_{P},\left(x, \mathcal{F}_{G}^{\prime}, \mathcal{F}_{M}^{\prime}, \widetilde{\kappa}_{P}^{\prime}\right) \in{ }_{x, 0} \widetilde{\operatorname{Bun}}_{P},\left(x, \mathcal{F}_{G}, \mathcal{F}_{G}^{\prime}, \beta\right) \in \mathcal{H}_{G}^{\lambda}$, $\left(x, \mathcal{F}_{M}, \mathcal{F}_{M}^{\prime}, \beta_{M}\right) \in \mathcal{H}_{M}^{\lambda}$ and the data of $\beta, \beta_{M}, \widetilde{\kappa}_{P}, \widetilde{\kappa}_{P}^{\prime}$ are compatible in the sense that over $X-x, \beta \circ \widetilde{\kappa}_{P}=\widetilde{\kappa}_{P}^{\prime} \circ \beta_{M}$.

We have the projections

$$
Z \rightarrow{ }_{r e l, 0} \widetilde{\operatorname{Bun}}_{P} \underset{\operatorname{Bun}_{M} \times X}{\times} \mathcal{H}_{M}^{\lambda} \text { and } Z \rightarrow{ }_{r e l, 0} \widetilde{\operatorname{Bun}}_{P} \underset{\operatorname{Bun}_{M} \times X}{\times} \mathcal{H}_{M}^{-\mathrm{w}_{0}^{M}(\lambda)},
$$

that send a point $z \in Z$ as above to

$$
\left(\left(x, \mathcal{F}_{G}, \mathcal{F}_{M}, \widetilde{\kappa}_{P}\right), \mathcal{F}_{M}^{\prime}, \beta_{M}\right) \text { and }\left(\left(x, \mathcal{F}_{G}^{\prime}, \mathcal{F}_{M}^{\prime}, \widetilde{\kappa}_{P}^{\prime}\right), \mathcal{F}_{M}, \beta_{M}^{-1}\right),
$$

respectively.

If follows from Sect. 4.3.6 that the first of the above projections is smooth, since the map $\mathfrak{t}_{P}^{\lambda}: S_{P}^{\lambda} \cap \mathrm{Gr}_{G}^{\lambda} \rightarrow \mathrm{Gr}_{M}^{\lambda}$ is smooth and that the second projection is an isomorphism, since the $\operatorname{map} \mathfrak{t}_{P}^{-\mathrm{w}_{0}^{M}(\lambda)}: S_{P}^{-\mathrm{w}_{0}^{M}(\lambda)} \cap \mathrm{Gr}_{G}^{-\mathrm{w}_{0}(\lambda)} \rightarrow \mathrm{Gr}_{M}^{-\mathrm{w}_{0}^{M}(\lambda)}$ is an isomorphism.

Let us denote by $\varphi_{1}$ and $\varphi_{0}$ the maps $Z \rightarrow \operatorname{Bun}_{o}$ that send $z \in Z$ as above to $\mathcal{F}_{M}$ and $\mathcal{F}_{M}^{\prime}$, respectively. Let $\stackrel{o}{Z}$ be the preimage of $\stackrel{o}{\operatorname{Bun}_{M}}$ under $\varphi_{1}$.

Lemma 5.3.8. $\mathrm{IC}_{Z}$ is ULA with respect to $\varphi_{2}: \stackrel{o}{Z} \rightarrow \operatorname{Bun}_{M}$.

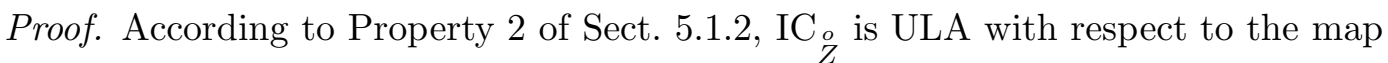
$\stackrel{o}{Z} \rightarrow{ }_{r e l, 0} \widetilde{\operatorname{Bun}}_{P} \underset{\operatorname{Bun}_{M} \times X}{\times} \mathcal{H}_{M}^{\lambda} \rightarrow \mathcal{H}_{M}^{\lambda}$.

Now, $\varphi_{2}$ is the composition $\stackrel{o}{Z} \rightarrow \mathcal{H}_{M}^{\lambda} \stackrel{h_{\vec{M}}}{\longrightarrow} \operatorname{Bun}_{M}$ and the assertion follows from Property 6 of Sect. 5.1.2.

Consider the map

$$
\stackrel{o}{Z} \bar{Z}^{\prime} \stackrel{h}{\vec{G}} \times \pi_{\longrightarrow}^{\longrightarrow} r e l, 0 \widetilde{\operatorname{Bun}}_{P} \rightarrow \widetilde{\operatorname{Bun}}_{P} .
$$

It is smooth and its composition with $\widetilde{\mathfrak{q}}_{P}: \widetilde{\operatorname{Bun}}_{P} \rightarrow \operatorname{Bun}_{M}$ equals $\varphi_{2}$. Therefore, in order to prove that $\mathrm{IC}_{\widetilde{\operatorname{Bun}_{P}}}$ is ULA with respect to $\widetilde{\mathfrak{q}}_{P}$ in a neighbourhood of $\mathcal{F}_{M}^{\prime} \in$ $\operatorname{Bun}_{M}\left(\overline{\mathbb{F}_{q}}\right)$, it is enough to show that the preimage $\widetilde{\mathfrak{q}}_{P}^{-1}\left(\mathcal{F}_{M}^{\prime}\right) \subset \widetilde{\operatorname{Bun}_{P}}\left(\overline{\mathbb{F}_{q}}\right)$ is contained in the image of $\stackrel{o}{Z}$ in $\widetilde{\operatorname{Bun}}_{P}$ under the above map.

Thus, let $\left(\mathcal{F}_{G}^{\prime}, \mathcal{F}_{M}^{\prime},\left\{\widetilde{\kappa}_{P}^{\prime} \mathcal{V}:\left(\mathcal{V}^{U(P)}\right)_{\mathcal{F}_{M}^{\prime}} \hookrightarrow \mathcal{V}_{\mathcal{F}_{G}^{\prime}}\right\}\right)$ be a $\overline{\mathbb{F}_{q}}$-point of $\widetilde{\mathfrak{q}}_{P}^{-1}\left(\mathcal{F}_{M}^{\prime}\right)$. As the map $\mathcal{H}_{M}^{\lambda} \stackrel{h_{M}}{\longrightarrow} \operatorname{Bun}_{M} \times X$ is smooth and, in particular, open, the fact that $\mathcal{F}_{M} \prec \mathcal{F}_{M}^{\prime}$ for some $\mathcal{F}_{M} \in \operatorname{Bun}_{M}\left(\overline{\mathbb{F}_{q}}\right)$, implies that there exists a triple $\left(\mathcal{F}_{M}^{\prime \prime}, x, \beta_{M}\right)$ such that

a) $\left(\mathcal{F}_{M}^{\prime \prime}, \mathcal{F}_{M}^{\prime}, x, \beta_{M}\right) \in \mathcal{H}_{M}^{\lambda}\left(\overline{\mathbb{F}_{q}}\right)$. 
b) The embeddings $\widetilde{\kappa}_{P}^{\prime \check{\lambda}}:\left(\mathcal{V}^{(U(P)}\right)_{\mathcal{F}_{M}^{\prime}} \hookrightarrow \mathcal{V}_{\mathcal{F}_{G}^{\prime}}$ have no zero at $x$.

c) $\mathcal{F}_{M}^{\prime \prime} \in \operatorname{Bun}_{M}\left(\overline{\mathbb{F}_{q}}\right)$.

But this exactly means that the point

$$
\left(x, \mathcal{F}_{G}^{\prime}, \mathcal{F}_{M}^{\prime}, \widetilde{\kappa}_{P}^{\prime}, \mathcal{F}_{M}^{\prime \prime}, \beta_{M}^{-1}\right) \in{ }_{r e l, 0} \widetilde{\operatorname{Bun}}_{P} \underset{\operatorname{Bun}_{M} \times X}{\times} \mathcal{H}_{M}^{-\mathrm{w}_{0}^{M}(\lambda)} \simeq Z
$$

belongs to $\stackrel{\circ}{Z}$.

The "only if" part of Proposition 5.3.4 follows from similar considerations by interchanging right and left.

\section{The Structure of Drinfeld's COMPaCtifications}

\subsection{Stratifications-I.}

6.1.1. Consider the set $\operatorname{Sym}^{\infty}(\Lambda)$ whose elements are finite unordered collections of elements of $\Lambda$ with possible repetitions. For

$$
\bar{\lambda}=\{\underbrace{\lambda_{1}, \ldots, \lambda_{1}}_{n_{1} \text { times }}, \ldots, \underbrace{\lambda_{k}, \ldots, \lambda_{k}}_{n_{k} \text { times }}\} \in \operatorname{Sym}^{\infty}(\Lambda),
$$

we define the corresponding partially symmetrized power of $X$ with all the diagonals removed as

$$
X^{\bar{\lambda}}:=X^{\left(n_{1}\right)} \times \ldots \times X^{\left(n_{k}\right)}-\Delta .
$$

To a point $\bar{x} \in X^{\bar{\lambda}}$, where $\bar{x}=x_{1,1}, \ldots, x_{1, n_{1}}, x_{2,1}, \ldots, x_{k, n_{k}}$ we will attach a $\Lambda$-valued divisor $\bar{\lambda} \cdot \bar{x}$ equal to $\Sigma \lambda_{i} \cdot x_{i, j}$. We will denote by $|\bar{\lambda}|$ the element of $\Lambda$ equal to $\Sigma n_{i} \cdot \lambda_{i}$.

Let $\bar{\lambda}$ belong to $\operatorname{Sym}^{\infty}\left(\Lambda_{G}^{\text {pos }}-0\right)$. Consider the map

$$
j_{\bar{\lambda}}: \operatorname{Bun}_{B} \times X^{\bar{\lambda}} \rightarrow \overline{\operatorname{Bun}}_{B}
$$

which sends a point $\left(\mathcal{F}_{G}, \mathcal{F}_{T}, \kappa\right) \times \bar{x}$ to $\left(\mathcal{F}_{G}, \mathcal{F}_{T}^{\prime}, \kappa^{\prime}\right)$, where $\mathcal{F}_{T}^{\prime}:=\mathcal{F}_{T}(-\bar{\lambda} \cdot \bar{x})$ and $\kappa^{\prime}$ corresponds to the composition:

$$
\mathcal{L}_{\mathcal{F}_{T}^{\prime}}^{\check{\lambda}} \hookrightarrow \mathcal{L}_{\mathcal{F}_{T}}^{\check{\lambda}} \stackrel{\kappa^{\grave{\lambda}}}{\longrightarrow} \mathcal{V}_{\mathcal{F}_{G}}^{\check{\lambda}}
$$

Proposition 6.1.2. For $\bar{\lambda} \in \operatorname{Sym}^{\infty}\left(\Lambda_{G}^{\mathrm{pos}}-0\right)$, the map $j_{\bar{\lambda}}$ a locally closed embedding.

Proof. The fact that $j_{\bar{\lambda}}$ is representable is evident, since both the source and the target are representable over $\operatorname{Bun}_{G}$.

Hence, for an $S$-point of $\overline{\mathrm{Bun}}_{B}$, its preimage under $j_{\bar{\lambda}}$ is a set, rather than a category, and to prove that $j_{\bar{\lambda}}$ is a locally closed embedding, one has to show that this set consists of at most one element. The latter is, however, obvious from the definitions.

Let ${ }_{\lambda} \operatorname{Bun}_{B}$ denote the locally closed substack of $\overline{\mathrm{Bun}}_{B}$ equal to the image of $j_{\bar{\lambda}}$. For a fixed point $\bar{x} \in X^{\bar{\lambda}}$, let $\overline{x, \bar{\lambda}} \operatorname{Bun}_{B}$ denote the image of $\operatorname{Bun}_{B} \times \bar{x}$ under $j_{\bar{\lambda}}$.

Therefore, by combining the above proposition with Proposition 1.2 .5 we obtain: 
Proposition 6.1.3. The locally closed substacks ${ }_{\lambda} \operatorname{Bun}_{B}$ form a stratification of $\overline{\operatorname{Bun}}_{B}$ as $\bar{\lambda}$ runs over $\operatorname{Sym}^{\infty}\left(\Lambda_{G}^{\text {pos }}-0\right)$.

6.1.4. Now let us give a proof of Proposition 1.2.3 which was announced in Sect. 1.2.1. For that end we need to introduce one more piece of notation. For $\bar{\lambda} \in \operatorname{Sym}^{\infty}\left(\Lambda_{G}^{+}\right)$, let $\mathcal{H}_{G}^{\bar{\lambda}}$ denote the relative version of the Hecke stack:

By definition, $\mathcal{H}_{G}^{\bar{\lambda}}$ is endowed with a map $\pi: \mathcal{H}_{G}^{\bar{\lambda}} \rightarrow X^{\bar{\lambda}}$ and its fiber over $\bar{x}=$ $x_{1,1}, \ldots, x_{1, n_{1}}, x_{2,1}, \ldots, x_{k, n_{k}}$ is the stack of triples $\left(\mathcal{F}_{G}, \mathcal{F}_{G}^{\prime}, \beta\right)$, where $\mathcal{F}_{G}$ and $\mathcal{F}_{G}^{\prime}$ are $G-$ bundles on $X$ and $\beta$ is an identification between them on $X-\{\bar{x}\}$ such that $\mathcal{F}_{G}^{\prime}$ is in position $\lambda_{i}$ with respect to $\mathcal{F}_{G}$ at $x_{i, j}$.

Let $h_{G}^{\overleftarrow{G}}$ and $h_{G}$ be the projections $\mathcal{H}_{G}^{\bar{\lambda}} \rightarrow \operatorname{Bun}_{G}$ that send $\left(\bar{x}, \mathcal{F}_{G}, \mathcal{F}_{G}^{\prime}, \beta\right)$ to $\mathcal{F}_{G}$ and $\mathcal{F}_{G}^{\prime}$, respectively. We will denote by $\bar{x} \mathcal{H}_{G}^{\bar{\lambda}}$ the fiber of $\mathcal{H}_{G}^{\bar{\lambda}}$ over $\bar{x} \in X^{\bar{\lambda}}$.

In a similar way we define the stacks $\overline{\mathcal{H}}_{G}^{\bar{\lambda}}$ and $\bar{x}_{\overline{\mathcal{H}}}^{\bar{\lambda}}$.

Proof. (of Proposition 1.2.3)

Let $\left(\mathcal{F}_{G}, \mathcal{F}_{T}, \kappa\right)$ be an $\overline{\mathbb{F}_{q}}$-point of $\overline{\operatorname{Bun}}_{B}$. To prove the proposition, it is enough to construct an irreducible stack $Z$ with a map $\phi: Z \rightarrow \overline{\mathrm{Bun}}_{B}$ such that

(a) $\operatorname{Im}(\phi) \cap \operatorname{Bun}_{B}$ is non-empty.

(b) $\left(\mathcal{F}_{G}, \mathcal{F}_{T}, \kappa\right) \in \operatorname{Im}(\phi)$.

Let $x_{1}, \ldots x_{n}$ be the set of points where $\left(\mathcal{F}_{G}, \mathcal{F}_{T}, \kappa\right)$ has singularities and let $\nu_{1}, \ldots, \nu_{n} \in$ $\Lambda_{G}^{\text {pos }}$ be the corresponding defects. Let us choose elements $\lambda_{1}, \ldots, \lambda_{n} \in \Lambda_{G}^{+}$in such a way that the weight spaces $V^{\lambda_{i}}\left(\mathrm{w}_{0}\left(\lambda_{i}\right)+\nu_{i}\right)$ are nonzero. The collection $\left\{\left(x_{1}, \lambda_{1}\right) \ldots,\left(x_{n}, \lambda_{n}\right)\right\}$ corresponds to a unique $\bar{\lambda} \in \operatorname{Sym}^{\infty}\left(\Lambda_{G}^{+}\right)$and $\bar{x} \in X^{\bar{\lambda}}$.

We define $Z^{\prime}$ as a fiber product $Z^{\prime}:=\bar{x}_{\bar{G}} \mathcal{H}_{G}^{\bar{\lambda}} \underset{\operatorname{Bun}_{G}}{\times} \operatorname{Bun}_{B}$, where $\bar{x}_{G}^{\bar{\lambda}}$ is mapped to $\operatorname{Bun}_{G}$ by means of $h_{G}$.

The stack $Z^{\prime}$ splits into connected components (which are in a bijection with the connected components of $\operatorname{Bun}_{B}$, and hence of $\operatorname{Bun}_{T}$ ) and we take $Z$ to be the connected component of $Z^{\prime}$ corresponding to the coweight $\operatorname{deg}\left(\mathcal{F}_{T}\right)-\mathrm{w}_{0}(|\bar{\lambda}|)$.

We define the map $\phi: Z^{\prime} \rightarrow \overline{\operatorname{Bun}}_{B}$ as in Sect. 3.1.1. Now, it follows from Lemma 3.3.6 that $Z$ satisfies conditions (a) and (b) above.

6.1.5. For an element $\lambda \in \Lambda_{G}^{\text {pos }}$ let us consider the following (semi-simple) complex $\operatorname{over} \operatorname{Spec}\left(\mathbb{F}_{q}\right)$ :

$$
\operatorname{Kost}_{\lambda}:=\underset{\lambda=\Sigma m_{\alpha} \cdot \alpha, \alpha \in \Delta^{+}}{\oplus}\left(\overline{\mathbb{Q}_{\ell}}(1)[2]\right)^{\otimes \Sigma m_{\alpha}},
$$

where the sum is taken over all the possible ways to represent $\lambda$ as a sum of positive coroots with non-negative coefficients. (This is a geometric counterpart of the $q$-analog of Kostant's partition function (cf. 20]).)

Theorem 6.1.6. For $\bar{\lambda}=\{\underbrace{\lambda_{1}, \ldots, \lambda_{1}}_{n_{1} \text { times }}, \ldots, \underbrace{\lambda_{k}, \ldots, \lambda_{k}}_{n_{k} \text { times }}\} \in \operatorname{Sym}^{\infty}\left(\Lambda^{\text {pos }}-0\right)$, the complex

$j_{\bar{\lambda}}^{*}\left(\mathrm{IC}_{\overline{\operatorname{Bun}}_{B}}\right)$ is isomorphic to $\left(\operatorname{IC}_{\mathrm{Bun}_{B}} \nabla \overline{\mathbb{Q}}_{X^{\bar{\lambda}}}\right) \underset{i=1}{\gtrless} \operatorname{Kost}_{\lambda_{i}}^{\otimes n_{i}}$. 
This result (along with its generalization, Theorem 6.2.9) will be proven in a subsequent publication. Now, let us show how Theorem 6.1.6 implies Theorem 2.2.11 (and hence Theorem 2.2.12):

Proof. (of Theorem 2.2.11)

For every collection of non-negative integers $\left\{m_{\alpha}\right\}, \alpha \in \Delta^{+}$, consider the corresponding map

$$
i_{\left\{m_{\alpha}\right\}}: \operatorname{Bun}_{B} \times \prod_{\alpha \in \Delta^{+}} X^{\left(m_{\alpha}\right)} \rightarrow \overline{\operatorname{Bun}}_{B} .
$$

Theorem 6.1.6 implies that the function on $\overline{\operatorname{Bun}}_{B}\left(\mathbb{F}_{q}\right)$ corresponding to the sheaf $\overline{\mathfrak{q}}^{! *}\left(\mathrm{Aut}_{E_{\tilde{T}}}^{\mu}\right)$ equals

$$
\sum_{\left\{m_{\alpha}\right\}} i_{\left\{m_{\alpha}\right\} !}\left(\operatorname{Funct}\left(\mathfrak{q}^{! *}\left(\operatorname{Aut}_{E_{\tilde{T}}}^{\mu-\Sigma m_{\alpha} \cdot \alpha}\right)\right) \otimes \prod_{\alpha \in \Delta^{+}} \operatorname{Funct}\left(\left(E_{\tilde{T}}^{\alpha}\right)^{\left(m_{\alpha}\right)} \otimes \overline{\mathbb{Q}_{\ell}}\left(m_{\alpha}\right)\right)\right) .
$$

By summing up along the fibers of the projection $\overline{\mathfrak{p}}$, we derive the formula of Theorem 2.2.11.

\subsection{Stratifications-II.}

6.2.1. Let us generalize the above discussion to the case of a parabolic subgroup $P$.

Conisider the set $\operatorname{Sym}^{\infty}\left(\Lambda_{G, P}^{\mathrm{pos}}-0\right)$ and for every element $\bar{\theta}=\{\underbrace{\theta_{1}, \ldots, \theta_{1}}_{n_{1} \text { times }}, \ldots, \underbrace{\theta_{k}, \ldots, \theta_{k}}_{n_{k} \text { times }}\}$

in it, consider the corresponding variety $X^{\bar{\theta}}=X^{\left(n_{1}\right)} \times \ldots \times X^{\left(n_{k}\right)}-\Delta$

As in Sect. 6.1.1, we have the natural locally closed embeddings

$$
j_{\bar{\theta}}: \operatorname{Bun}_{P} \times X^{\bar{\theta}} \hookrightarrow \overline{\operatorname{Bun}}_{P}
$$

and for a fixed $\bar{\theta} \in \operatorname{Sym}^{\infty}\left(\Lambda_{G, P}^{\text {pos }}-0\right)$ (resp., $\bar{x} \in X^{\bar{\theta}}$ ) we will denote by $\bar{\theta}_{\theta}$ Bun $_{P}$ (resp., $\bar{x}, \bar{\theta} \operatorname{Bun}_{P}$ ) the corresponding stratum (resp., locally closed subset) of $\overline{\mathrm{Bun}}_{P}$.

Thus, for $\bar{\theta}$ and $\bar{x}$ as above we obtain the locally closed substacks $\mathfrak{r}_{P}^{-1}\left({ }_{\theta} \operatorname{Bun}_{P}\right)$ and $\mathfrak{r}_{P}^{-1}\left(\bar{x}_{\bar{\theta}} \operatorname{Bun}_{P}\right)$ of $\widetilde{\operatorname{Bun}}_{P}$. However, it will not be true that $\mathrm{IC}_{\widetilde{\operatorname{Bun}_{P}}}$ is smooth when restricted to the strata of the form $\mathfrak{r}_{P}^{-1}\left({ }_{\bar{\theta}} \operatorname{Bun}_{P}\right)$. Our next goal is to define a suitable refinement of this stratification.

6.2.2. Consider the affine Grassmannian of the group $M$ and let $\mathrm{Gr}_{M}^{+} \subset \mathrm{Gr}_{M}$ be a closed subscheme definied by the following condition:

$\mathcal{F}_{M} \in \mathrm{Gr}_{M}^{+}$if for every $G$-module $\mathcal{V}$, the map

$$
\left.\beta_{M}^{\mathcal{\mathcal { V } ^ { ( U ( P ) }}:\left(\mathcal{V}^{(U(P)}\right)}\right)\left.\left._{\mathcal{F}_{M}}\right|_{\mathcal{D}_{x}^{*}} \rightarrow\left(\mathcal{V}^{(U(P)}\right)_{\mathcal{F}_{M}^{0}}\right|_{\mathcal{D}_{x}^{*}}
$$

is regular on $\mathcal{D}_{x}$.

It is clear from the definition, that $\mathrm{Gr}_{M}^{+}$is stable under the $M\left(\mathcal{O}_{x}\right)$-action on $\mathrm{Gr}_{M}$.

Proposition 6.2.3. For an $M$ co-weight $\nu \in \Lambda_{M}^{+}$the following conditions are equivalent:

(a) $\overline{\mathrm{Gr}}_{M}^{\nu} \subset \mathrm{Gr}_{M}^{+}$.

(b) $\mathrm{w}_{0}^{M}(\nu) \in \Lambda_{G}^{\mathrm{pos}}$. 
Proof. Assume that $\overline{\mathrm{Gr}}_{M}^{\nu} \subset \mathrm{Gr}_{M}^{+}$. In particular, the corresponding point $\mathcal{F}_{M}:=t_{x}^{\nu} \in$ $M\left(\mathcal{K}_{x}\right) / M\left(\mathcal{O}_{x}\right)=\operatorname{Gr}_{M}$ belongs to $\mathrm{Gr}_{M}^{+}$. By definition, we must have that for $\check{\lambda} \in \Lambda_{G}^{+}$ the map

$$
\left.\left.\mathcal{U}_{\mathcal{F}_{M}}^{\check{\lambda}}\right|_{\mathcal{D}_{x}^{*}} \rightarrow \mathcal{U}_{\mathcal{F}_{M}^{0}}^{\check{\lambda}}\right|_{\mathcal{D}_{x}^{*}}
$$

is regular on $\mathcal{D}_{x}$, which implies that $\left\langle\nu, \mathrm{w}_{0}^{M}(\check{\lambda})\right\rangle \geq 0$. The latter inequality means exactly that $\mathrm{w}_{0}^{M}(\nu) \in \Lambda_{G}^{\text {pos }}$.

Conversely, let us assume that $\mathrm{w}_{0}^{M}(\nu) \in \Lambda_{G}^{\text {pos }}$. Let $\mathcal{U}$ be an $M$-module of the form $\mathcal{V}^{U(P)}$, where $\mathcal{V}$ is a $G$-module. Without restricting the generality, we way assume that the weights of $\mathcal{V}$ are $\leq \check{\lambda}$ for some $\check{\lambda} \in \Lambda_{G}^{+}$. Then the weights of $\mathcal{U}$ are $\leq \check{M} \check{\lambda}$. Hence, by definition, $\mathcal{F}_{M} \in \overline{\mathrm{Gr}}_{M}^{\nu}$ means that

$$
\beta_{M}^{\mathcal{U}}: \mathcal{U}_{\mathcal{F}_{M}} \rightarrow \mathcal{U}_{\mathcal{F}_{M}^{0}}\left(-\left\langle\mathrm{w}_{0}^{M}(\nu), \check{\lambda}\right\rangle\right)
$$

However, $\left\langle\mathrm{w}_{0}^{M}(\nu), \check{\lambda}\right\rangle \geq 0$, by assumption.

In what follows we will denote by $\Lambda_{M, G}^{+} \subset \Lambda_{M}^{+}$the above sub-semigroup, i.e. $\Lambda_{M, G}^{+}=$ $\Lambda_{M}^{+} \cap \mathrm{w}_{0}^{M}\left(\Lambda_{G}^{\mathrm{pos}}\right)$. For example, for $M=T, \Lambda_{T, G}^{+}=\Lambda_{G}^{\text {pos }}$. In general, the projection $\Lambda \rightarrow \Lambda_{G, P}$ sends $\Lambda_{M, G}^{+}$to $\Lambda_{G, P}^{\text {pos }}$.

Let now $\theta$ be an element of $\Lambda_{G, P}^{\text {pos }}$. We define the element $b(\theta) \in \Lambda_{M, G}^{+}$as follows: By definition, $\theta$ is the projection under $\Lambda \rightarrow \Lambda_{G, P}$ of some $\sum_{\mathfrak{i} \in \mathcal{J}_{-} \mathcal{J}_{M}} b_{\mathfrak{i}} \cdot \alpha_{\mathfrak{i}}$. We set $b(\theta)=\mathrm{w}_{0}^{M}\left(\sum_{\mathfrak{i} \in \mathcal{J}_{-} \mathcal{J}_{M}} b_{\mathfrak{i}} \cdot \alpha_{\mathfrak{i}}\right)$. It belongs to $\mathrm{w}_{0}^{M}\left(\Lambda_{G}^{\text {pos }}\right)$ by construction, and to $\Lambda_{M}^{+}$, because every $\alpha_{\mathfrak{i}}, \mathfrak{i} \notin \mathcal{J}_{M}$ is $M$-antidominant.

For $\theta$ as above let $\operatorname{Gr}_{M}^{\theta}$ denote the corresponding connected component of $\mathrm{Gr}_{M}$. Set $\mathrm{Gr}_{M}^{+, \theta}:=\mathrm{Gr}_{M}^{+} \cap \mathrm{Gr}_{M}^{\theta}$.

From the above proposition it follows that $b(\theta)$ is the maximal element in the set of $\nu \in \Lambda_{M}^{+}$such that $\overline{\mathrm{Gr}}_{M}^{\nu} \subset \mathrm{Gr}_{M}^{+} \cap \mathrm{Gr}_{M}^{\theta}$. Hence, we obtain:

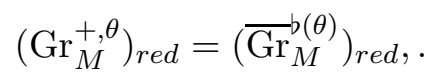

where the subscript "red" means "the corresponding reduced scheme".

6.2.4. Fix $\bar{\theta}=\{\underbrace{\theta_{1}, \ldots, \theta_{1}}_{n_{1} \text { times }}, \ldots, \underbrace{\theta_{k}, \ldots, \theta_{k}}_{n_{k} \text { times }}\} \in \operatorname{Sym}^{\infty}\left(\Lambda_{G, P}^{\text {pos }}-0\right)$. Let us denote by $\mathcal{H}_{M}^{+, \bar{\theta}}$ a version of the Hecke stack, which is fibered over $X^{\bar{\theta}}$ with the fiber over a point $\bar{x} \in X^{\bar{\theta}}$ given by $x_{1,1}, \ldots, x_{1, n_{1}}, x_{2,1}, \ldots, x_{k, n_{k}}$ being the product

$$
x_{1,1} \mathcal{H}_{M}^{+, \theta_{1}} \underset{\operatorname{Bun}_{M}}{\times} \underset{\operatorname{Bun}_{M}}{\times} x_{k, n_{k}} \mathcal{H}_{M}^{+, \theta_{k}} .
$$




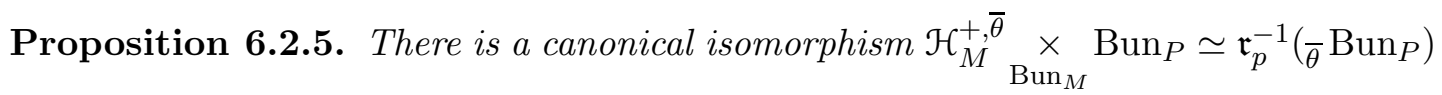
that fits into the commutative diagram

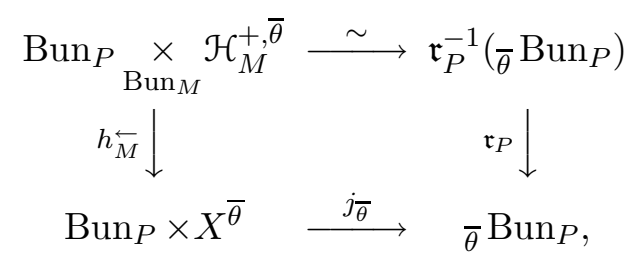

where we have used the projection $h_{M}^{\leftarrow}: \mathcal{H}_{M}^{+, \bar{\theta}} \rightarrow \operatorname{Bun}_{M}$ to define the fiber product.

Proof. Let $\left(\bar{x}, \mathcal{F}_{P}, \mathcal{F}_{M}^{\prime}, \beta_{M}\right)$ be a point of $\operatorname{Bun}_{P} \underset{\operatorname{Bun}_{M}}{\times} \mathcal{H}_{M}^{+, \bar{\theta}}$, where $\mathcal{F}_{P}$ is a $P$-bundle on $X$ and $\beta_{M}$ is an isomorphism between $\mathcal{F}_{M}:=U(P) \backslash \mathcal{F}_{P}$ and $\mathcal{F}_{M}^{\prime}$ defined outside of $\{\bar{x}\} \subset X$.

We attach to it a point of $\mathfrak{r}_{P}^{-1}\left({ }_{\bar{\theta}} \mathrm{Bun}_{P}\right)$ as follows:

The corresponding $G$-bundle is induced from $\mathcal{F}_{P}$, i.e. $\mathcal{F}_{G}:=\mathcal{F}_{P} \stackrel{P}{\times} G$ and the corresponding $M$-bundle is $\mathcal{F}_{M}^{\prime}$. Now, the data of $\widetilde{\kappa}_{P}^{\prime}{ }^{\prime}:\left(\mathcal{V}^{U(P)}\right)_{\mathcal{F}_{M}^{\prime}} \rightarrow \mathcal{V}_{\mathcal{F}_{G}}$ is obtained as a composition

$$
\left(\mathcal{V}^{U(P)}\right)_{\mathcal{F}_{M}^{\prime}} \stackrel{\beta_{M}^{\nu^{U(P)}}}{\hookrightarrow}\left(\mathcal{V}^{U(P)}\right)_{\mathcal{F}_{M}} \rightarrow \mathcal{V}_{\mathcal{F}_{G}}
$$

Conversely, let $\left(\mathcal{F}_{G}, \mathcal{F}_{M}^{\prime}, \widetilde{\kappa}_{P}\right)$ be an $(S)$-point of $\mathfrak{r}_{P}^{-1}\left({ }_{\bar{\theta}} \mathrm{Bun}_{P}\right)$. Let $\left(\bar{x}, \mathcal{F}_{P}\right)$ be the corresponding point of $\operatorname{Bun}_{P} \times X^{\bar{\theta}}$, in particular, let $\mathcal{F}_{M}$ be the corresponding $M$ bundle. By definition, $\mathcal{F}_{M}$ and $\mathcal{F}_{M}^{\prime}$ are identified outside $\bar{x}$.

By assumption, for each $G$-module $\mathcal{V}$, we have the embedding:

$$
\widetilde{\kappa}_{P}^{\prime \mathcal{V}}:\left(\mathcal{V}^{U(P)}\right)_{\mathcal{F}_{M}^{\prime}} \hookrightarrow \mathcal{V}_{\mathcal{F}_{G}} .
$$

and the maximal embedding

$$
\widetilde{\kappa}_{P}^{\mathcal{V}}:\left(\mathcal{V}^{U(P)}\right)_{\mathcal{F}_{M}} \hookrightarrow \mathcal{V}_{\mathcal{F}_{G}}
$$

Hence, the (a priori) meromorphic map map $\left(\mathcal{V}^{U(P)}\right)_{\mathcal{F}_{M}^{\prime}} \rightarrow\left(\mathcal{V}^{U(P)}\right)_{\mathcal{F}_{M}}$ is regular.

6.2.6. If $\bar{\nu}$ is an element of $\operatorname{Sym}^{\infty}\left(\Lambda_{M, G}^{+}-0\right)$, we obtain a locally closed embedding

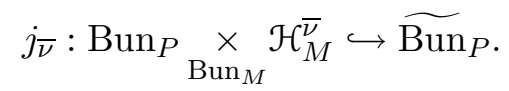

Its image will be denoted by ${ }_{\nu} \operatorname{Bun}_{P}$, and for $\bar{x} \in X^{\bar{\nu}}$ we let $\bar{x}_{\bar{\nu}} \operatorname{Bun}_{P}$ denote the corresponding closed substack of ${ }_{\nu} \mathrm{Bun}_{P}$.

Thus, we see that the defect of a point $\left(\mathcal{F}_{G}, \mathcal{F}_{M}^{\prime}, \widetilde{\kappa}_{P}\right) \in \widetilde{\operatorname{Bun}_{P}}\left(\overline{\mathbb{F}_{q}}\right)$ at a point of $X$ where it has a singularity is naturally an element of $\Lambda_{M, G}^{+}$. However, unlike the case of $\overline{\operatorname{Bun}}_{B}$ and $\overline{\operatorname{Bun}}_{P}$, fixing the locus of singularities of an enhanced parabolic Drinfeld's structure, together with the defects and the saturated $P$-bundle, does not determine 
the corresponding point of $\widetilde{\operatorname{Bun}}_{P}$ uniquely: we have the remaining freedom of choosing a point in the product of the corresponding $\overline{\mathrm{Gr}}_{M}^{+, \theta}$ 's.

At this point we are ready to give a proof of Proposition 1.3.7.

Proof. (of Proposition 1.3.7)

As we have seen above, the fibers of the map $\mathfrak{r}_{P}: \widetilde{\operatorname{Bun}}_{P} \rightarrow \overline{\operatorname{Bun}}_{P}$ are never empty. Therefore, it is enough to prove that $\operatorname{Bun}_{P}$ is dense in $\widetilde{\operatorname{Bun}} P$. Let $\left(\mathcal{F}_{G}, \mathcal{F}_{M}, \widetilde{\kappa}_{P}\right)$ be an $\overline{\mathbb{F}} q$-point of $\widetilde{\operatorname{Bun}}_{P}$.

As in the case of Proposition 1.2.3, it is enough to construct an irreducible stack $Z$ with a map $\phi: Z \rightarrow \widetilde{\operatorname{Bun}}_{P}$, whose image contains $\left(\mathcal{F}_{G}, \mathcal{F}_{M}, \widetilde{\kappa}_{P}\right)$ and such that $\operatorname{Im}(\phi) \cap \operatorname{Bun}_{P} \neq \emptyset$.

To simplify the notation, we will assume that $\left(\mathcal{F}_{G}, \mathcal{F}_{M}, \widetilde{\kappa}_{P}\right)$ has a singularity only at one point, call it $x$. Let the defect be $\nu \in \Lambda_{M, G}^{+}$. We have: $\left(\mathcal{F}_{G}, \mathcal{F}_{M}, \widetilde{\kappa}_{P}\right) \subset{ }_{x, \nu} \widetilde{\operatorname{Bun}}_{P}$, in the terminology of Sect. 4.2.1.

Let $\lambda \in \Lambda_{G}^{+}$be such that: (a) $\left\langle\mathrm{w}_{0}(\lambda), \check{\alpha}_{\mathfrak{i}}\right\rangle=0$ for $\mathfrak{i} \in \mathcal{J}_{M} ;(\mathrm{b}) \operatorname{Hom}_{\check{M}}\left(U^{\mathrm{w}_{0}(\lambda)+\nu}, V^{\lambda}\right) \neq$ 0 . It is easy to see that such $\lambda$ indeed exists (cf. Lemma 6.2.8). Note that condition (a) means that $\mathrm{w}_{0}(\lambda)$ lies in the group of cocharacters of $Z^{0}(M)$.

Set $Z^{\prime}:={ }_{x} \mathcal{H}_{G}^{\lambda} \underset{\operatorname{Bun}_{G}}{\times} \operatorname{Bun}_{P}$, where ${ }_{x} \mathcal{H}_{G}^{\lambda}$ is mapped to Bun ${ }_{G}$ by means of $h_{G}$. We define the map $\varphi: Z^{\prime} \rightarrow{\widetilde{\operatorname{Bun}_{P}}}_{\text {as follows: }}$

For a point $\left(\mathcal{F}_{G}, \beta, \mathcal{F}_{G}^{\prime}, \mathcal{F}_{M}^{\prime}, \widetilde{\kappa}_{P}^{\prime}\right) \in Z^{\prime}$, the resulting $G$-bundle is $\mathcal{F}_{G}$, the $M$-bundle is $\mathcal{F}_{M}^{\prime} \otimes \mathcal{F}_{Z^{0}(M)}^{0}\left(\mathrm{w}_{0}(\lambda) \cdot x\right)$ and the new $\widetilde{\kappa}_{P}$ are obtained from $\widetilde{\kappa}_{P}^{\prime}$ as in Sect. 3.1.1.

We define $Z$ as the preimage in $Z^{\prime}$ of the appropriate connected component of $\operatorname{Bun}_{P}$. The fact that $Z$ satisfies the required properties follows from Sect. 4.3.6.

6.2.7. Here we will describe the behaviour of $\mathrm{IC}_{\widetilde{\mathrm{Bun}}_{P}}$ along the strata ${ }_{\nu} \mathrm{Bun}_{P}$ introduced above.

Recall (cf. Theorem 3.2.8) that to every representaion $U$ of the group $\check{M}$ we can attach in a canonical way a $M\left(\mathcal{O}_{x}\right)$-equivariant perverse sheaf $F_{M}^{-1}(U)$ on the affine Grassmannian $\operatorname{Gr}_{M}$. For $\theta \in \Lambda_{G, P}^{\text {pos }}$ we introduce the following semi-simple complex on $\mathrm{Gr}_{M}$ :

$$
\operatorname{Kost}_{\theta}^{P}:=\underset{i \geq 0}{\oplus} F_{M}^{-1}\left(\operatorname{Sym}^{i}\left(\check{\mathfrak{u}}_{P}\right)_{\theta}\right) \otimes \overline{\mathbb{Q}_{\ell}}(i)[2 i],
$$

where $\check{\mathfrak{u}}_{P}$ denotes the unipotent radical of the corresponding parabolic in $\check{G}$ and where for an $\check{M}$-representation $U, U_{\theta}$ denotes its piece on which $Z(\check{M})$ acts by the character $\theta$.

Lemma 6.2.8. For an $M$-dominant coweight $\nu, \operatorname{Hom}_{\check{M}}\left(U^{\nu}, \operatorname{Sym}^{i}\left(\check{\mathfrak{u}}_{P}\right)_{\theta}\right) \neq 0$ if and only if $\nu \in \Lambda_{M, G}^{+}$. Moreover, the above space is non-zero only for finitely many integers $i$.

Therefore, $\operatorname{Kost}_{\theta}^{P}$ is supported on $\overline{\operatorname{Gr}}_{M}^{b(\theta)} \subset \operatorname{Gr}_{M}^{+, \theta}$. Analogously, for $\bar{\theta} \in \operatorname{Sym}^{\infty}\left(\Lambda_{G, P}^{\text {pos }}-\right.$ $0)$ we define the semi-simple complex $\operatorname{Kost}_{\bar{\theta}}$ on $\mathcal{H}_{M}^{+, \bar{\theta}}$. 
Theorem 6.2.9. Under the identification

$$
\operatorname{Bun}_{P} \underset{\operatorname{Bun}_{M}}{\times} \mathcal{H}_{M}^{+, \bar{\theta}}
$$

the sheaf $\left.\mathrm{IC}_{\widetilde{\mathrm{Bun}_{P}}}\right|_{\widetilde{\operatorname{Bun}}_{P}}$ goes over to $\mathrm{IC}_{\mathrm{Bun}_{P}} \otimes \operatorname{Kost} \frac{P}{\theta}$.

This theorem will be neither used nor proven in this paper (the proof will appear in [7]). For our purposes the following weaker result will be sufficient:

Theorem 6.2.10. For fixed $\bar{\nu} \in \operatorname{Sym}^{\infty}\left(\Lambda_{M, G}^{+}-0\right)$ and $\bar{x} \in X^{\bar{\nu}}$, the $*$-restriction of $\mathrm{IC}_{\mathrm{Bun}_{P}}$ to $\bar{x}, \bar{\nu} \operatorname{Bun}_{P}$ is locally constant.

The proof will occupy the next subsection. The reader will notice that it is very similar to the proof of Theorem 5.1.5.

\subsection{Proof of Theorem 6.2.10.}

6.3.1. Consider the fiber product $\bar{Z}={ }_{y} \mathcal{H}_{G}^{\lambda} \underset{\operatorname{Bun}_{G}}{\times} \operatorname{Bun}_{P}$, where $y$ is a point in $X-\{\bar{x}\}$, $\lambda$ is some element of $\Lambda_{G}^{+}$and the fibered product is formed using the projection $h_{G}$ : ${ }_{y} \mathcal{H}_{G}^{\lambda} \rightarrow \operatorname{Bun}_{G}$.

As before, there is a projection ${ }^{\prime} h_{G}^{\overleftarrow{Z}}: \bar{Z} \rightarrow{ }_{y, \infty} \widetilde{\operatorname{Bun}}_{P}$ and we will denote by $Z$ the locally closed substack of $\bar{Z}$ defined as

$$
\left.Z:={ }^{\prime} h_{G}^{\leftarrow}{ }^{-1}{ }_{y, \lambda} \widetilde{\operatorname{Bun}}_{P}\right) \cap^{\prime} h_{G}^{-1}\left({ }_{y, 0} \widetilde{\operatorname{Bun}}_{P}\right)
$$

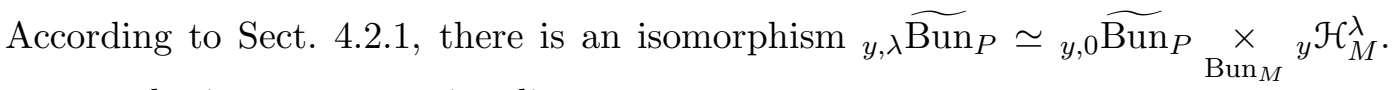
Hence we obtain a commutative diagram:

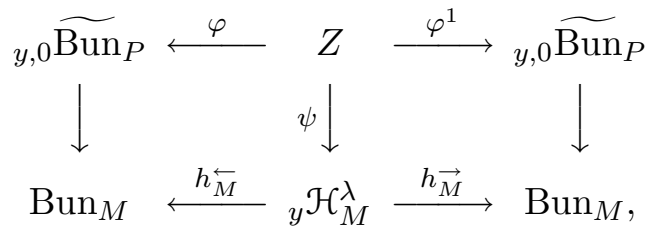

where both upper horizontal arrows are smooth. Moreover, the preimages of $\bar{x}, \bar{\nu} \operatorname{Bun}_{P}$ in $Z$ under the maps $\varphi$ and $\varphi^{1}$ coincide.

Consider two $\overline{\mathbb{F}_{q}}$-points of the stack $\overline{x, \bar{\nu}} \operatorname{Bun}_{P}$. Using Proposition 6.2 .5 , they can be represented by two triples $\left(\mathcal{F}_{P}, \mathcal{F}_{M}^{\prime}, \beta\right)$ and $\left(\mathcal{F}_{P}^{1}, \mathcal{F}_{M}^{1}{ }^{\prime}, \beta^{1}\right)$, where $\beta$ is an isomorphism $\left.\left.\mathcal{F}_{M}\right|_{X-\{\bar{x}\}} \rightarrow \mathcal{F}_{M}^{\prime}\right|_{X-\{\bar{x}\}}$ (here $\mathcal{F}_{M}$ is the $M$-bundle induced from $\mathcal{F}_{P}$ ), such that at every $x_{i, j}, \mathcal{F}_{M}^{\prime}$ is in position $\nu_{i}$ with respect to $\mathcal{F}_{M}$; and similarly for $\beta^{1}$.

We will write that $\left(\mathcal{F}_{P}, \mathcal{F}_{M}^{\prime}, \beta\right) \succ\left(\mathcal{F}_{P}^{1}, \mathcal{F}_{M}^{1}{ }^{\prime}, \beta^{1}\right)$ if there exist $y \in X-\{\bar{x}\}, \lambda \in \Lambda_{G}^{+}$ and $z \in Z$ as above such that $\left(\mathcal{F}_{P}, \mathcal{F}_{M}^{\prime}, \beta\right)=\varphi(z),\left(\mathcal{F}_{P}^{1}, \mathcal{F}_{M}^{1}{ }^{\prime}, \beta^{1}\right)=\varphi^{1}(z)$. Consider the equivalence relation $\sim$ on the set of $\overline{\mathbb{F}_{q}}$-points of ${ }_{\bar{x}, \bar{\nu}} \operatorname{Bun}_{P}$ generated by $\succ$.

We will deduce the assertion of Theorem 6.2.10 from the following proposition:

Proposition 6.3.2. All $\overline{\mathbb{F}_{q}}$-points of $\overline{x, \bar{\nu}} \operatorname{Bun}_{P}$ are $\sim$-equivalent. 
Proof. Set $\widetilde{M}:=M / Z^{0}(M)$ and for a an $M$-bundle, we will denote by a subscript $\widetilde{M}$ the corresponding induced $\widetilde{M}$-bundle.

Step 1. Let $\left(\mathcal{F}_{P}, \mathcal{F}_{M}^{\prime}, \beta\right)$ and $\left(\mathcal{F}_{P}^{1}, \mathcal{F}_{M}^{1}{ }^{\prime}, \beta^{1}\right)$ be two $\overline{\mathbb{F}_{q}}$-points of ${ }_{\bar{x}, \bar{\nu}} \operatorname{Bun}_{P}$. First we will show that $\left(\mathcal{F}_{P}^{1}, \mathcal{F}_{M}^{1}{ }^{\prime}, \beta^{1}\right)$ is $\sim$-equivalent to another $\overline{\mathbb{F}_{q}}$-point $\left(\mathcal{F}_{P}^{2}, \mathcal{F}_{M}^{2}{ }^{\prime}, \beta^{2}\right)$, such that $\mathcal{F}_{\widetilde{M}} \simeq \mathcal{F}_{\widetilde{M}}^{2}$ and the induced isomorphism

$$
\left.\left.\mathcal{F}_{\widetilde{M}^{\prime}}\right|_{X-\{\bar{x}\}} \simeq \mathcal{F}_{\widetilde{M}}^{2}\right|_{X-\{\bar{x}\}}
$$

is regular on the whole of $X$.

Pick $y \notin\{\bar{x}\}$. As $\widetilde{M}$ is semi-simple, the $\widetilde{M}$-bundles $\mathcal{F}_{\widetilde{M}}$ and $\mathcal{F}_{\widetilde{M}}^{1}$ are isomorphic over $X-y$ (cf. Lemma 5.3.6). Moreover, since $X-y$ is affine, the group of automorphisms of $\mathcal{F}_{\widetilde{M}}$ over $X-y$ is dense in $\prod_{i, j} G\left(\mathcal{O}_{x_{i, j}}\right)\left(\overline{\mathbb{F}_{q}}\right)$. Therefore, since at every $x_{i, j}$ the relative position of $\mathcal{F}_{M}^{\prime}$ with respect to $\mathcal{F}_{M}$ is the same as the relative position of $\mathcal{F}_{M}^{1}{ }^{\prime}$ with respect to $\mathcal{F}_{M}^{1}$, the above isomorphism $\left.\left.\mathcal{F}_{\widetilde{M}}\right|_{X-y} \rightarrow \mathcal{F}_{\widetilde{M}}^{1}\right|_{X-y}$ can be chosen in such a way that the isomorphism between $\left.\mathcal{F}_{\widetilde{M}}^{\prime}\right|_{X-\{\bar{x}, y\}}$ and $\left.\mathcal{F}_{\widetilde{M}}^{1}\right|_{X-\{\bar{x}, y\}}$ is regular on $X-y$.

Consider the corresponding point $\left(\mathcal{F}_{\widetilde{M}}, \mathcal{F}_{\widetilde{M}}^{1}, \beta_{\widetilde{M}}\right) \in{ }_{y} \mathcal{H}_{\widetilde{M}}^{\nu}$. Let $\lambda \in \Lambda_{G}^{+}$be such that it projects onto $\nu$ under $\Lambda \rightarrow \Lambda_{\widetilde{M}}$ and consider the corresponding stack $Z$. The composition

$$
Z \stackrel{\psi \times \varphi_{1}}{\longrightarrow} y \mathcal{H}_{M}^{\lambda} \underset{\operatorname{Bun}_{M}}{\times}{ }_{y, 0} \widetilde{\operatorname{Bun}}_{P} \simeq{ }_{y} \mathcal{H}_{\widetilde{M}}^{\nu} \underset{\operatorname{Bun}_{\widetilde{M}}}{\times} \underset{y, 0}{\widetilde{\operatorname{Bun}}_{P}}
$$

is surjective and let $z \in Z$ be such that under the above composition it maps to the point

$$
\left(\mathcal{F}_{\widetilde{M}},\left(\mathcal{F}_{P}^{1}, \mathcal{F}_{M}^{1}{ }^{\prime}, \beta^{1}\right), \beta_{\widetilde{M}}:\left.\left.\mathcal{F}_{\widetilde{M}}\right|_{X-y} \simeq \mathcal{F}_{\widetilde{M}}^{1}\right|_{X-y}\right) \in_{y} \mathcal{H}_{\widetilde{M}}^{\nu} \underset{\operatorname{Bun}_{\widetilde{M}}}{\times} \underset{y, 0}{\operatorname{Bun}_{P}} .
$$

We define $\left(\mathcal{F}_{P}^{2}, \mathcal{F}_{M}^{2}{ }^{\prime}, \beta^{2}\right)$ as the image of $z$ in under the projection $\varphi$. By construction, it satisfies the required condition.

Step 2. Using the assertion of Step 1, we can assume that there exists an isomorphism $\mathcal{F}_{\widetilde{M}} \rightarrow \mathcal{F}_{\widetilde{M}}^{1}$ which gives rise to an isomorphism $\mathcal{F}_{\widetilde{M}}^{\prime} \rightarrow \mathcal{F}_{\widetilde{M}}^{1}{ }^{\prime}$.

For a $\Lambda_{Z^{0}(M)}$-divisor $D$ and an $M$-bundle $\mathcal{F}_{M}$, let $\mathcal{F}_{M}(D)$ denote the new $M$-bundle $\mathcal{F}_{M} \otimes \mathcal{F}_{Z^{0}(M)}^{0}(D)$.

It is clear that we can choose $\Lambda_{Z^{0}(M)} \cap \Lambda_{G^{-v a l u e d}}^{+}$divisors $D$ and $D_{1}$ on $X-\{\bar{x}\}$, so that there exists an isomorphism $\mathcal{F}_{M}(D) \simeq \mathcal{F}_{M}^{1}\left(D_{1}\right)$. In this case, the identification $\left.\left.\mathcal{F}_{M}^{\prime}(D)\right|_{X-\{\bar{x}\}} \simeq \mathcal{F}_{M}^{1}{ }^{\prime}\left(D_{1}\right)\right|_{X-\{\bar{x}\}}$ is automatically regular on the whole of $X$.

Therefore, by arguing as above, we can replace our two points $\left(\mathcal{F}_{P}, \mathcal{F}_{M}^{\prime}, \beta\right)$ and $\left(\mathcal{F}_{P}^{1}, \mathcal{F}_{M}^{1}{ }^{\prime}, \beta_{1}\right)$ by $\sim$-equivalent points (we will abuse the notation and denote the latter by the same characters) such that there exists an isomorphism $\mathcal{F}_{M} \rightarrow \mathcal{F}_{M}^{1}$, for which the induced meromorphic map $\left.\left.\mathcal{F}_{M}^{\prime}\right|_{X-\{\bar{x}\}} \rightarrow \mathcal{F}_{M}^{1}\right|_{X-\{\bar{x}\}}$ is a global isomorphism.

Moreover, by replacing $D$ and $D_{1}$ by $D+D^{\prime}$ and $D_{1}+D^{\prime}$, where $D^{\prime} \in \Lambda_{Z^{0}(M)} \cap \Lambda_{G}^{+}$ is sufficiently large, we can ensure that the cohomology

$$
H^{1}\left(X, \mathcal{U}_{\mathcal{F}_{M}}\right)=0
$$


for all irreducible $M$-modules $\mathcal{U}$ which appear in the Jordan-Holder series of the Lie algebra $\mathfrak{u}(P)$, viewed as a $P$-module via the adjoint action.

However, (7) implies that any two $P$-bundles, whose reductions modulo $U(P)$ are isomorphic to $\mathcal{F}_{M}$, are necessarily isomorphic. This implies that our two points $\left(\mathcal{F}_{P}, \mathcal{F}_{M}^{\prime}, \beta\right)$ and $\left(\mathcal{F}_{P}^{1}, \mathcal{F}_{M}^{1}{ }^{\prime}, \beta_{1}\right)$ are isomorphic and, in particular, $\sim$-equivalent.

\subsubsection{Now let us prove Theorem 6.2.10:}

Proof. Let $\left(\mathcal{F}_{P}, \mathcal{F}_{M}, \beta\right)$ and $\left(\mathcal{F}_{P}^{1}, \mathcal{F}_{M}^{1}{ }^{\prime}, \beta^{1}\right)$ be two $\overline{\mathbb{F}_{q}}$-points of $\overline{x, \bar{\nu}}$ Bun $_{P}$. Let us denote by $\iota: \operatorname{Spec}\left(\overline{\mathbb{F}_{q}}\right) \rightarrow \bar{x}, \bar{\nu} \operatorname{Bun}_{P}$ (resp., $\iota_{1}$ ) the corresponding map. Consider the complexes

$$
\iota^{*}\left(\left.\mathrm{IC}_{\widetilde{\operatorname{Bun}_{P}}}\right|_{\bar{x}, \bar{\nu} \operatorname{Bun}_{P}}\right), \iota_{1}^{*}\left(\left.\mathrm{IC}_{\widetilde{\operatorname{Bun}_{P}}}\right|_{\bar{x}, \bar{\nu} \operatorname{Bun}_{P}}\right), \iota^{!}\left(\left.\mathrm{IC}_{\widetilde{\operatorname{Bun}_{P}}}\right|_{\bar{x}, \bar{\nu} \operatorname{Bun}_{P}}\right), \iota_{1} !\left(\left.\mathrm{IC}_{\widetilde{\operatorname{Bun}_{P}}}\right|_{\bar{x}, \bar{\nu}} \operatorname{Bun}_{P}\right)
$$

over $\operatorname{Spec}\left(\overline{\mathbb{F}_{q}}\right)$. We will normalize (by making a cohomological shift and Tate's twist) in such a way that their highest (resp., lowest) cohomology is $\overline{\mathbb{Q}_{\ell}}$ in degree 0 .

To prove the theorem, it is enough to show that for any $\left(\mathcal{F}_{P}, \mathcal{F}_{M}, \beta\right)$ and $\left(\mathcal{F}_{P}^{1}, \mathcal{F}_{M}^{1}{ }^{\prime}, \beta^{1}\right)$ as above,

$$
\begin{aligned}
& \iota^{*}\left(\left.\mathrm{IC}_{\widetilde{\operatorname{Bun}_{P}}}\right|_{\bar{x}, \bar{\nu} \operatorname{Bun}_{P}}\right) \simeq \iota_{1}^{*}\left(\left.\mathrm{IC}_{\widetilde{\operatorname{Bun}_{P}}}\right|_{\bar{x}, \bar{\nu} \operatorname{Bun}_{P}}\right) \\
& \iota^{!}\left(\left.\mathrm{IC}_{\widetilde{\operatorname{Bun}_{P}}}\right|_{\bar{x}, \bar{\nu} \operatorname{Bun}_{P}}\right) \simeq \iota_{1} !\left(\left.\mathrm{IC}_{\widetilde{\operatorname{Bun}_{P}}}\right|_{\bar{x}, \bar{\nu}} \operatorname{Bun}_{P}\right) .
\end{aligned}
$$

Using Proposition 6.3.2, we can assume that $\left(\mathcal{F}_{P}, \mathcal{F}_{M}^{\prime}, \beta\right) \succ\left(\mathcal{F}_{P}^{1}, \mathcal{F}_{M}^{1}{ }^{\prime}, \beta^{1}\right)$ and let $\iota_{z}: \operatorname{Spec}\left(\overline{\mathbb{F}_{q}}\right) \rightarrow Z$ be a point such that $\varphi \circ \iota_{z}=\iota, \varphi^{1} \circ \iota_{z}=\iota_{1}$. Let $\bar{x}, \bar{\nu} Z$ denote the (common) preimage of $\overline{x, \bar{\nu}} \operatorname{Bun}_{P}$ in $Z$ under $\varphi$ or $\varphi^{1}$.

Since both maps $\varphi$ and $\varphi_{1}$ are smooth we have:

$$
\begin{aligned}
& \iota^{*}\left(\left.\mathrm{IC}_{\widetilde{\operatorname{Bun}_{P}}}\right|_{\bar{x}, \bar{\nu}} \operatorname{Bun}_{P}\right) \simeq \iota_{z}^{*}\left(\left.\mathrm{IC}_{Z}\right|_{\bar{x}, \bar{\nu} Z}\right) \simeq \iota_{1}^{*}\left(\left.\mathrm{IC}_{\widetilde{\operatorname{Bun}_{P}}}\right|_{\bar{x}, \bar{\nu}} \operatorname{Bun}_{P}\right) \text { and } \\
& \iota^{!}\left(\left.\mathrm{IC}_{\widetilde{\operatorname{Bun}_{P}}}\right|_{\bar{x}, \bar{\nu}} \operatorname{Bun}_{P}\right) \simeq \iota_{z}^{!}\left(\left.\mathrm{IC}_{Z}\right|_{\bar{x}, \bar{\nu}} Z \simeq \iota_{1} !\left(\left.\mathrm{IC}_{\widetilde{\operatorname{Bun}_{P}}}\right|_{\bar{x}, \bar{\nu}} \operatorname{Bun}_{P}\right),\right.
\end{aligned}
$$

where $\iota_{z}^{*}(?)$ and $\iota_{z}^{!}(?)$ are also normalized in the above way.

This proves our assertion.

Proposition 6.3.2 implies also the following strengthening of Theorem 6.2.10:

Corollary 6.3.4. Let $K$ be an irreducible subquotient of an $i$-th perverse cohomology sheaf $h^{i}\left(\left.\mathrm{IC}_{\widetilde{\mathrm{Bun}_{P}}}\right|_{\bar{\nu}} \operatorname{Bun}_{P}\right)$. Then $K$ is constant along the fibers of the projection $\bar{\nu}_{\bar{B}} \operatorname{Bun}_{P} \rightarrow$ $X^{\bar{\nu}}$.

Proof. It is enough to prove that for each $\bar{x} \in X^{\bar{\nu}}$, every irreducible subquotient of $h^{i}\left(\left.\mathrm{IC}_{\widetilde{\operatorname{Bun}}_{P}}\right|_{\bar{x}, \bar{\nu}} \operatorname{Bun}_{P}\right)$ is a constant sheaf on $\overline{x, \nu} \operatorname{Bun}_{P}$. Therefore, it suffices to show that $\operatorname{Funct}\left(h^{i}\left(\left.\mathrm{IC}_{\widetilde{\operatorname{Bun}_{P}}}\right|_{\bar{x}, \bar{\nu}} \operatorname{Bun}_{P}\right)\right)$ is a constant function on $\overline{x, \bar{\nu}} \operatorname{Bun}_{P}\left(\mathbb{F}_{q^{\prime}}\right)$ for all $\mathbb{F}_{q^{\prime}} \supset \mathbb{F}_{q}$.

For that purpose, consider two points $\iota^{\prime}, \iota_{1}^{\prime}: \operatorname{Spec}\left(\mathbb{F}_{q^{\prime}}\right) \rightarrow \bar{x}, \bar{\nu} \operatorname{Bun}_{P}$ and for an extension $\mathbb{F}_{q^{\prime \prime}} \supset \mathbb{F}_{q^{\prime}}$, let $\iota^{\prime \prime}$ and $\iota_{1}^{\prime \prime}$ denote the corresponding $\mathbb{F}_{q^{\prime \prime}}-$ points of $\bar{x}, \bar{\nu} \operatorname{Bun}_{P}$.

As in the above proof of Theorem 6.2.10, we infer from Proposition 6.3.2 that when $\mathbb{F}_{q^{\prime \prime}}$ is sufficiently large, the sheaves

$$
\iota^{\prime \prime *}\left(h^{i}\left(\left.\mathrm{IC}_{\widetilde{\operatorname{Bun}_{P}}}\right|_{\bar{x}, \bar{\nu}} \operatorname{Bun}_{P}\right)\right) \text { and } \iota_{1}^{\prime \prime *}\left(h^{i}\left(\left.\mathrm{IC}_{\widetilde{\operatorname{Bun}_{P}}}\right|_{\bar{x}, \bar{\nu}} \operatorname{Bun}_{P}\right)\right)
$$


over $\operatorname{Spec}\left(\mathbb{F}_{q^{\prime \prime}}\right)$ are isomorphic.

Since this is true for infinitely many $q^{\prime \prime}$, we obtain that

$$
\operatorname{Tr}\left(F r, \iota^{\prime *}\left(h^{i}\left(\left.\mathrm{IC}_{\widetilde{\operatorname{Bun}_{P}}}\right|_{\bar{x}, \bar{\nu}} \operatorname{Bun}_{P}\right)\right)\right)=\operatorname{Tr}\left(F r, \iota_{1}^{\prime *}\left(h^{i}\left(\left.\mathrm{IC}_{\widetilde{\operatorname{Bun}}_{P}}\right|_{\bar{x}, \bar{\nu}} \operatorname{Bun}_{P}\right)\right)\right)
$$

which is what we had to prove.

\section{4. "Good" perverse sheaves and Theorem 2.3.4.}

6.4.1. In this subsection our goal is to prove Theorem 2.3.4, which was announced in Sect. 2.3. We shall start with the following general observation:

Proposition 6.4.2. For $\mathcal{S} \in \operatorname{Bun}_{G}$ and $H_{G}^{\lambda}$ as in Sect. 2.1.4, the sheaf $H_{G}^{\lambda}(\mathcal{S})$ on $\operatorname{Bun}_{G} \times X$ is ULA with respect to the projection $\operatorname{Bun}_{G} \times X \rightarrow X$.

Proof. We have:

$$
H_{G}^{\lambda}(\mathcal{S}):=\left(h_{G}^{\overleftarrow{G}} \times \pi\right)_{!}\left(h_{G}^{*}(\mathcal{S}) \otimes \mathrm{IC}_{\overline{\mathcal{H}}_{G}^{\lambda}}\right) \otimes\left(\overline{\mathbb{Q}_{\ell}}\left(\frac{1}{2}\right)[1]\right)^{-\operatorname{dim}\left(\operatorname{Bun}_{G}\right)}
$$

As the projection $h_{G}^{\leftarrow} \times \pi: \overline{\mathcal{H}}^{\lambda} \rightarrow \operatorname{Bun}_{G} \times X$ is proper, it follows from Property 3 of Sect. 5.1.2 that it is enough to show that the sheaf $h_{G}^{*}(\mathcal{S}) \otimes \mathrm{IC}_{\overline{\mathcal{H}}_{G}^{\lambda}}$ on $\overline{\mathcal{H}}_{G}^{\lambda}$ is ULA with repect to the projection $\pi: \overline{\mathcal{H}}_{G}^{\lambda} \rightarrow X$.

However, since $\overline{\mathcal{H}}_{G}^{\lambda} \stackrel{h_{\vec{G}} \times \pi}{\longrightarrow} \operatorname{Bun}_{G} \times X$ is a fibration (locally trivial in the smooth topology), the needed assertion follows from Property 1 of Sect. 5.1.2.

In the same way as above, it is easy to prove that for any $\mathcal{S} \in \operatorname{Sh}\left(\operatorname{Bun}_{G}\right)$ and a sequence $\lambda_{1}, \ldots, \lambda_{n}$ of elements of $\Lambda_{G}^{+}$, the sheaf

$$
\left(H_{G}^{\lambda_{n}} \otimes \mathrm{id}^{n-1}\right) \circ \ldots \circ\left(H_{G}^{\lambda_{2}} \otimes \mathrm{id}\right) \circ H_{G}^{\lambda_{1}}(\mathcal{S})
$$

on $\operatorname{Bun}_{G} \times X^{n}$ is ULA with respect to the projection $\operatorname{Bun}_{G} \times X^{n} \rightarrow \operatorname{Bun}_{G}$.

Now let $\bar{\lambda}$ be an element of $\operatorname{Sym}^{\infty}\left(\Lambda_{G}^{+}-0\right)$ equal to $\{\underbrace{\lambda_{1}, \ldots, \lambda_{1}}_{n_{1} \text { times }}, \ldots, \underbrace{\lambda_{k}, \ldots, \lambda_{k}}_{n_{k} \text { times }}\}$. Set $N=\Sigma n_{i}$ and consider the functor $\stackrel{o}{H}_{G}^{\bar{\lambda}}: \operatorname{Sh}\left(\operatorname{Bun}_{G}\right) \rightarrow \operatorname{Sh}\left(\operatorname{Bun}_{G} \times X^{\bar{\lambda}}\right)$ given by

$$
\mathcal{S} \rightarrow\left(h_{G}^{\leftarrow} \times \pi\right) ! \circ h_{G}^{*}(\mathcal{S})[\langle|\bar{\lambda}|, 2 \check{\rho}\rangle+N]
$$

defined using the stack $\mathcal{H}_{G}^{\bar{\lambda}}$.

Proposition 6.4.3. Let $\mathcal{S}$ be a "good" perverse sheaf on $\operatorname{Bun}_{G}$. Then $\stackrel{o}{H}_{G}^{\bar{\lambda}}(\mathcal{S})$ lies in cohomological degrees $\leq 0$ and is ULA with respect to the projection $\operatorname{Sh}\left(\operatorname{Bun}_{G} \times X^{\bar{\lambda}}\right) \rightarrow$ $X^{\bar{\lambda}}$.

Proof. Let $\bar{\lambda}$ be equal to $\{\underbrace{\lambda_{1}, \ldots, \lambda_{1}}_{n_{1} \text { times }}, \ldots, \underbrace{\lambda_{k}, \ldots, \lambda_{k}}_{n_{k} \text { times }}\}$. Consider the non-symmetrized Hecke stack ${ }^{\prime} \mathcal{H}_{G}^{\bar{\lambda}}$, defined as ${ }^{\prime} \mathcal{H}_{G}^{\bar{\lambda}}:={ }^{\prime} X^{\bar{\lambda}} \underset{X^{\bar{\lambda}}}{\times} \mathcal{H}_{G}^{\bar{\lambda}}$, where ${ }^{\prime} X^{\bar{\lambda}}:=X^{n_{1}} \times \ldots \times X^{n_{k}}-\Delta$. 
Let ${ }^{\prime} h_{G} \vec{G}, h_{G}^{\overleftarrow{G}}$ and ${ }^{\prime} \pi$ denote the projections from ${ }^{\prime} \mathcal{H}_{G}^{\bar{\lambda}}$ to Bun $_{G}$ and ${ }^{\prime} X^{\bar{\lambda}}$, respectively. The map ${ }^{\prime} X^{\bar{\lambda}} \rightarrow X^{\bar{\lambda}}$ is an étale covering. Therefore, it is enough to show that the complex

$$
{ }^{\prime} \stackrel{o}{G}_{G}^{\bar{\lambda}}(\mathcal{S}):=\left({ }^{\prime} h_{G} \times^{\prime} \pi\right) ! \circ^{\prime} h_{G} \vec{G}^{*}(\mathcal{S})[\langle|\bar{\lambda}|, 2 \check{\rho}\rangle+N] \in \operatorname{Sh}\left(\operatorname{Bun}_{G} \times^{\prime} X^{\bar{\lambda}}\right)
$$

lives in non-positive cohomological degrees and is ULA with respect to the projection $\operatorname{Bun}_{G} \times^{\prime} X^{\bar{\lambda}} \rightarrow{ }^{\prime} X^{\bar{\lambda}}$.

Observe that the sheaf $\overline{\mathbb{Q}}_{\mathrm{Gr}^{\lambda}}[\langle\lambda, 2 \check{\rho}\rangle]$ on the affine Grassmannian is an extention of sheaves of the form $\mathcal{A}_{G}^{\lambda^{\prime}}[m], \lambda^{\prime} \leq \lambda, m \geq 0$. Hence, the complex ${ }^{\prime} H_{G}^{\bar{\lambda}}(\mathcal{S})$ is an extention of complexes of the form $\left(H_{G}^{\lambda_{N}^{\prime}} \otimes \mathrm{id}^{N-1}\right) \circ \ldots \circ\left(H_{G}^{\lambda_{2}^{\prime}} \otimes \mathrm{id}\right) \circ H_{G}^{\lambda_{1}^{\prime}}(\mathcal{S})[m]$ for finitely many values of $\lambda_{1}^{\prime}, \ldots, \lambda_{N}^{\prime}$ and $m \geq 0$.

This proves the required assertion in view of the definition of "good"' perverse sheaves and the above Proposition 6.4.2.

\subsubsection{Now we can prove Theorem 2.3.4.}

Proof. It is clear that if $\mathcal{S}$ is a "good" perverse sheaf on $\operatorname{Bun}_{M}$, then so is $\mathbb{D}(\mathcal{S})$. Therefore, to prove the theorem we have to show that the restriction of $\mathfrak{r}_{P !} \circ \widetilde{\mathfrak{q}}_{P}^{! *}(\mathcal{S})$ to $\overline{\mathrm{Bun}}_{P}-\operatorname{Bun}_{P}$ lives in negative cohomological degrees if $S$ is "good". In other words, we must show that for every $\bar{\nu} \in \operatorname{Sym}^{\infty}\left(\Lambda_{M, G}^{+}-0\right)$, the sheaf

$$
\mathfrak{r}_{P !} \circ j_{\bar{\nu} !} \circ j_{\bar{\nu}}^{*} \circ \widetilde{\mathfrak{q}}_{P}^{*}(\mathcal{S})
$$

lives in negative cohomological degrees.

For $\bar{\nu}$ as above, let $\bar{\theta}$ be the corresponding element of $\operatorname{Sym}^{\infty}\left(\Lambda_{G, P}^{\text {pos }}-0\right)$. The map $\mathfrak{r}_{P} \circ j_{\bar{\nu}}:{ }_{\nu} \operatorname{Bun}_{P} \simeq \operatorname{Bun}_{P} \underset{\operatorname{Bun}_{M}}{\times \mathcal{H}_{M}^{\bar{\nu}}} \rightarrow{ }_{\theta} \operatorname{Bun}_{P}$ is the long vertical map in the diagram

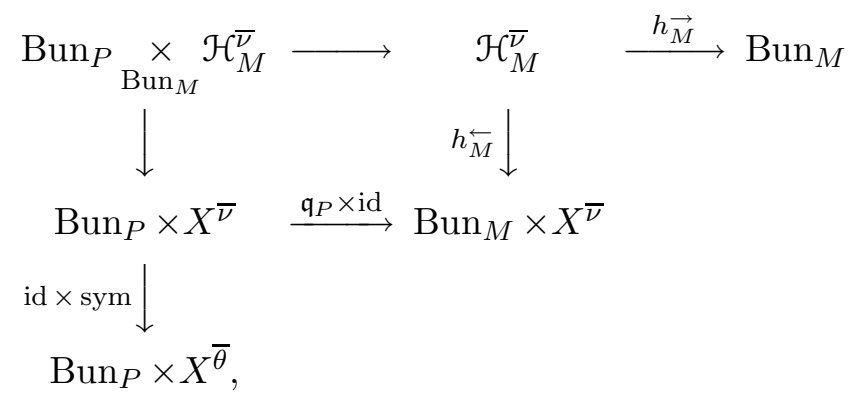

where the map $X^{\bar{\nu}} \rightarrow X^{\bar{\theta}}$ is a finite (symmetrization) map. Moreover, the long horizontal map in this diagram is nothing but $\widetilde{\mathfrak{q}}_{P} \circ j_{\bar{\nu}}$.

Let $K$ be an irreducible subquotient of $h^{i}\left(\left.\mathrm{IC}_{\widetilde{\operatorname{Bun}_{P}}}\right|_{\nu} \operatorname{Bun}_{P}\right)$ for some $i \in \mathbb{N}$ (of course, $i<0)$. Corollary 6.3.4 implies that every such $K$ is a pull-back of $K^{\prime}\left[N^{\prime}+\left\langle|\bar{\nu}|, 2 \check{\rho}_{M}\right\rangle\right]$, where $K^{\prime}$ is a perverse sheaf on $X^{\bar{\nu}}$ and $N^{\prime}$ is the dimension of the corresponding connected component of $\operatorname{Bun}_{P}$. 
By applying the projection formula, we obtain that $\mathfrak{r}_{P !} \circ j_{\bar{\nu} !} \circ j_{\nu}^{*} \circ \widetilde{\mathfrak{q}}_{P}^{! *}(\mathcal{S})$ is an extention of sheaves of the form

$$
(\mathrm{id} \times \operatorname{sym}) ! \circ\left(\mathfrak{q}_{P} \times \mathrm{id}\right)^{*}\left(\left(\overline{\mathbb{Q}}_{\ell} \operatorname{Bun}_{M} \otimes K^{\prime}\right) \otimes \stackrel{o}{H_{M}^{\bar{\nu}}}(\mathcal{S})\right)\left[-N+N^{\prime}-\operatorname{dim}\left(\operatorname{Bun}_{M}\right)+i\right],
$$

where $N$ is as in Proposition 6.4 .3 above and $i>0$.

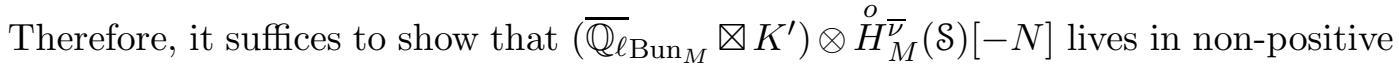
cohomological degrees. However, this follows immediately from Proposition 6.4.3 and Property 5 of Sect. 5.1.2.

\section{The Functional equation}

7.1. Theorem 7.1.6. In this subsection we will formulate and prove Theorem 7.1.6 which is one of the main results of this paper.

7.1.1. Let us consider the following stack

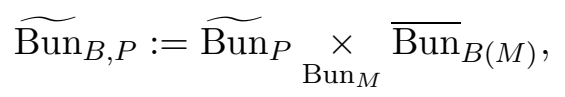

where $B(M)$ is the Borel subgroup of the group $M$ :

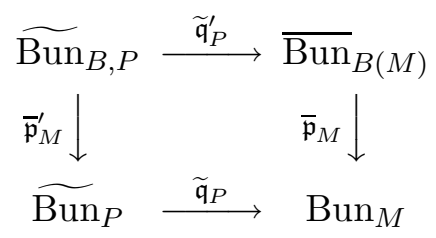

and let $\mathcal{C}\left(\mathrm{IC}_{\widetilde{\operatorname{Bun}_{P}}}, \mathrm{IC}_{\overline{\operatorname{Bun}}_{B(M)}}\right)$ denote the object

$$
\overline{\mathfrak{p}}_{M}^{\prime}\left(\mathrm{IC}_{\overline{\operatorname{Bun}}_{B(M)}}\right) \otimes \widetilde{\mathfrak{q}}_{P}^{\prime *}\left(\mathrm{IC}_{\widetilde{\operatorname{Bun}} P}\right) \otimes\left(\overline{\mathbb{Q}_{\ell}}\left(\frac{1}{2}\right)[1]\right)^{\otimes-\operatorname{dim}\left(\operatorname{Bun}_{M}\right)} \in \operatorname{Sh}\left(\widetilde{\operatorname{Bun}}_{B, P}\right) .
$$

Theorem 7.1.2. There sheaf $\mathrm{C}\left(\mathrm{IC}_{\mathrm{Bun}_{P}}, \mathrm{IC}_{\overline{\mathrm{Bun}}_{B(M)}}\right)$ is canonically isomorphic to the intersection cohomology sheaf of $\widetilde{\operatorname{Bun}}_{B, P}$.

Proof. Consider the following general set-up:

Let $f: Y_{1} \rightarrow Y_{2}$ be a map of algebraic stacks with $Y_{2}$ smooth. Let $j: Y_{1}^{0} \hookrightarrow Y_{1}$ be an open substack such that the map $f \circ j: Y_{1}^{0} \rightarrow Y_{2}$ is smooth as well. Assume, in addition, that the sheaves $\mathrm{IC}_{Y_{1}}$ and $j_{!}\left(\mathrm{IC}_{Y_{1}^{0}}\right)$ are ULA with respect to the map $f$.

Now let $Y_{3}$ be another algebraic stack mapping to $Y_{2}$. Let $Y^{\prime}$ be the Cartesian product:

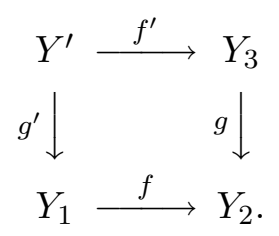


Lemma 7.1.3. In the above situation

$$
\mathrm{IC}_{Y^{\prime}} \simeq f^{\prime *}\left(\mathrm{IC}_{Y_{3}}\right) \otimes g^{\prime *}\left(\mathrm{IC}_{Y_{1}}\right) \otimes\left(\overline{\mathbb{Q}_{\ell}}\left(\frac{1}{2}\right)[1]\right)^{\otimes-\operatorname{dim}\left(Y_{2}\right)} .
$$

The proof of this lemma follows immediately from the definition of the ULA property (cf. proof of Theorem 2.3.2 in Sect. 5.1.4).

We apply this lemma in the situation when $Y_{2}:=\operatorname{Bun}_{M}, Y_{1}:=\widetilde{\operatorname{Bun}}_{P}, Y_{3}:=\overline{\operatorname{Bun}}_{B(M)}$ and the assertion of our theorem follows from Theorem 5.1.5.

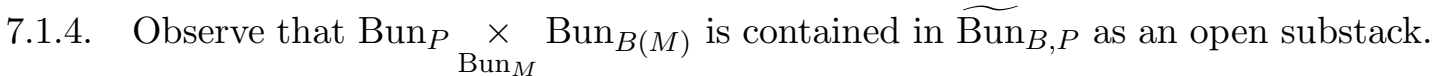

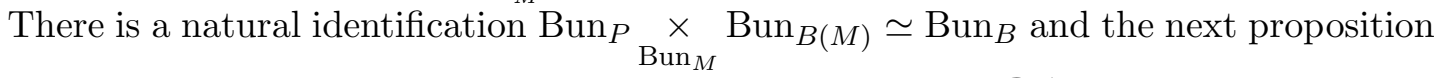
says that the map in one direction extends to the whole of $\widetilde{\operatorname{Bun}}_{B, P}$ :

Proposition 7.1.5. There exists a natural map $\mathfrak{k}_{P}: \widetilde{\operatorname{Bun}}_{B, P} \rightarrow \overline{\operatorname{Bun}}_{B}$, which is representable and proper.

Proof. A point of the stack $\widetilde{\operatorname{Bun}}_{B, P}$ is by definition a triple of bundles $\left(\mathcal{F}_{G}, \mathcal{F}_{M}, \mathcal{F}_{T}\right)$ plus a collection of embeddings

$$
\begin{aligned}
& \widetilde{\kappa}_{P}^{\mathcal{V}}:\left(\mathcal{V}^{U(P)}\right)_{\mathcal{F}_{M}} \hookrightarrow \mathcal{V}_{\mathcal{F}_{G}} \text { for all } G \text {-modules } \mathcal{V}, \\
& \kappa_{M}^{\check{\nu}}: \mathcal{L}_{\mathcal{F}_{T}}^{\check{L}} \rightarrow U_{\mathcal{F}_{M}}^{\check{\nu}}, \forall \nu \in \Lambda_{M}^{+},
\end{aligned}
$$

which satisfy the Plücker relations.

We set: $\mathfrak{k}_{P}\left(\mathcal{F}_{G}, \mathcal{F}_{M}, \mathcal{F}_{T}, \widetilde{\kappa}_{P}, \kappa_{M}\right)=\left(\mathcal{F}_{G}, \mathcal{F}_{T}, \kappa\right)$, where for $\check{\lambda} \in \check{\Lambda}_{G}^{+}$, the map $\kappa^{\check{\lambda}}$ is the composition:

$$
\mathcal{L}_{\mathcal{F}_{T}}^{\check{\lambda}} \stackrel{\kappa_{M}^{\check{\lambda}}}{\longrightarrow} \mathcal{U}_{\mathcal{F}_{M}}^{\check{\lambda}} \rightarrow\left(\left(\mathcal{V}^{\check{\lambda}}\right)^{U(P)}\right)_{\mathcal{F}_{M}} \stackrel{\widetilde{\kappa}_{P}^{\check{\lambda}}}{\longrightarrow} \mathcal{V}_{\mathcal{F}_{G}}^{\check{\lambda}}
$$

Theorem 7.1.6. $\mathfrak{k}_{P !}\left(\mathcal{C}\left(\mathrm{IC}_{\overline{\mathrm{Bun}}_{P}}, \mathrm{IC}_{\overline{\mathrm{Bun}}_{B(M)}}\right)\right) \simeq \mathrm{IC}_{\overline{\mathrm{Bun}}_{B}}$.

The rest of this subsection will be devoted to the proof of this theorem.

7.1.7. It is obvious that over $\operatorname{Bun}_{B} \subset \overline{\operatorname{Bun}}_{B}$, the sheaves $\mathfrak{k}_{P !}\left(\mathrm{C}\left(\mathrm{IC}_{\widetilde{\operatorname{Bun}}_{P}}, \operatorname{IC}_{\overline{\operatorname{Bun}}_{B(M)}}\right)\right)$ and $\mathrm{IC}_{\overline{\mathrm{Bun}}_{B}}$ coincide. Since the map $\mathfrak{k}_{P}$ is proper, it follows from Theorem 7.1.2 that in order to prove Theorem 7.1.6, it suffices to check that the $*$-restriction of the sheaf $\mathfrak{k}_{P !}\left(\mathrm{e}\left(\mathrm{IC}_{\widetilde{\operatorname{Bun}}_{P}}, \mathrm{IC}_{\overline{\operatorname{Bun}}_{B(M)}}\right)\right)$ to $\overline{\mathrm{Bun}}_{B}-\mathrm{Bun}_{B}$ lives in negative cohomological degrees.

Consider the set $\operatorname{Sym}^{\infty}\left(\left(\Lambda_{M}^{\text {pos }} \times \Lambda_{M, G}^{+}\right)-0\right)$, whose elements we will symbolically denote by $\overline{\mu, \nu}$, each being of the form

$$
\{\underbrace{\left(\mu_{1} \times \nu_{1}\right), \ldots,\left(\mu_{1} \times \nu_{1}\right)}_{n_{1} \text { times }}, \ldots, \underbrace{\left(\mu_{k} \times \nu_{k}\right), \ldots,\left(\mu_{k} \times \nu_{k}\right)}_{n_{k} \text { times }}\}
$$

where in every pair $\left(\mu_{1} \times \nu_{i}\right)$ one of the elements is non-zero. We set $|\overline{\mu, \nu}|:=\sum_{i=1, \ldots, k} n_{i}$. $\left(\nu_{i}-\mu_{i}\right) \in \Lambda$. 
Let $X^{\overline{\mu, \nu}}$ be the corresponding partially symmetrized power of $X$ and let $\mathcal{H}_{M}^{\overline{\mu, \nu}} \stackrel{\pi}{\rightarrow}$ $X^{\overline{\mu, \nu}}$ be the appropriate version of the Hecke stack. The constructions of Sect. 6.1 and Sect. 6.2 yield naturally defined maps:

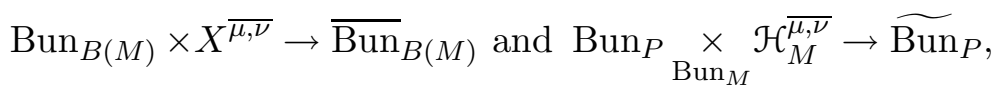

each being a composition of a smooth map followed by a locally closed embedding. (The smooth part appears due to the fact that some of the $\mu_{i}$ 's and $\nu_{i}$ 's may vanish or coincide.) We will denote their images by $\overline{\mu, \nu} \operatorname{Bun}_{B(M)}$ and $\overline{\mu, \nu} \operatorname{Bun}_{P}$, respectively. Finally, we will denote by $\overline{\mu, \nu} \operatorname{Bun}_{B, P}$ the intersection

$$
\overline{\mathfrak{p}}_{M}^{\prime-1}\left(\overline{\mu, \nu} \operatorname{Bun}_{P}\right) \cap \widetilde{\mathfrak{q}}_{P}^{\prime-1}\left(\overline{\mu, \nu} \operatorname{Bun}_{B(M)}\right) \subset \widetilde{\operatorname{Bun}}_{B, P}
$$

By definition, $\overline{\mu, \nu} \operatorname{Bun}_{B, P}$ is the image of a locally closed embedding

$$
\operatorname{Bun}_{P} \underset{\operatorname{Bun}_{M}}{\times \mathcal{H}_{M}^{\overline{\mu, \nu}}} \underset{\operatorname{Bun}_{M}}{\times} \operatorname{Bun}_{B(M)} \hookrightarrow \widetilde{\operatorname{Bun}}_{B, P}
$$

where the fiber product is formed using the map $\left(h_{M}^{\overleftarrow{M}} \times h_{M}\right): \mathcal{H}_{M}^{\overline{\mu, \nu}} \rightarrow \operatorname{Bun}_{M} \times \operatorname{Bun}_{M}$.

Now, for $\lambda \in \Lambda_{G}^{\text {pos }}$, let $\overline{\operatorname{Bun}}_{B}^{\lambda}$ be the locally closed substack of $\overline{\operatorname{Bun}}_{B}$ defined as a union of all ${ }_{\lambda} \operatorname{Bun}_{B}$ with $|\bar{\lambda}|=\lambda$. For $\overline{\mu, \nu}$ as above, we set $\overline{\mu, \nu}, \lambda \operatorname{Bun}_{B, P}$ to be the

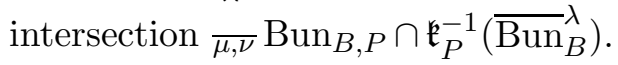

To prove Theorem 7.1.6, it suffices to check the following:

Proposition 7.1.8. For $\overline{\mu, \nu}$ and $\lambda$ as above the following holds:

(a) The *-restriction of $\mathrm{C}\left(\mathrm{IC}_{\widetilde{\mathrm{Bun}_{P}}}, \mathrm{IC}_{\left.\overline{\mathrm{Bun}}_{B(M)}\right)}\right.$ to $\overline{\mu, \nu, \lambda} \operatorname{Bun}_{B, P}$ lives in the cohomological degrees strictly smaller than $-\left\langle\lambda+|\overline{\mu, \nu}|, \check{\rho}_{M}\right\rangle$.

(b) The fibers of the map $\mathfrak{k}_{P}: \overline{\mu, \nu},{ }_{\mathrm{Bun}} \widetilde{\mathrm{B}, P}_{\lambda} \rightarrow{ }_{\lambda} \overline{\mathrm{Bun}}_{B}$ are of dimension $\leq\left\langle\lambda+|\overline{\mu, \nu}|, \check{\rho}_{M}\right\rangle$.

7.1.9. Proof of Proposition 7.1.8. For $\overline{\mu, \nu}$ as above set $N=\Sigma n_{i}$ and consider the corresponding symmetrization map $X^{N}-\Delta \simeq{ }^{\prime} X^{\overline{\mu, \nu}} \rightarrow X^{\overline{\mu, \nu}}$. We will introduce an order in our $\overline{\mu, \nu}$ and write it as $\left(\mu_{1}, \nu_{1}\right), \ldots,\left(\mu_{j}, \nu_{j}\right), \ldots,\left(\mu_{N}, \nu_{N}\right)$. Let ' $\mathcal{H}_{M}^{\overline{\mu, \nu}}$ be the corresponding desymmetrized Hecke stack, i.e. its fiber over $\left(x_{1}, \ldots, x_{N}\right) \in{ }^{\prime} X^{\overline{\mu, \nu}}$ is the fiber product of ${ }_{x_{j}} \mathcal{H}_{M}^{\nu_{j}}, j=1, \ldots, N$ over $\operatorname{Bun}_{M}$, with respect to the projections

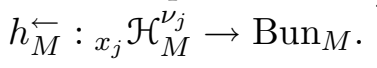

For a collection of elements $\lambda_{1}, \ldots, \lambda_{N}$ of $\Lambda_{G}^{+}$let $Z^{\left\{\mu_{j}\right\},\left\{\nu_{j}\right\},\left\{\lambda_{j}\right\}}$ denote the following locally closed substack of $\operatorname{Bun}_{P} \underset{\operatorname{Bun}_{M}}{\times} \mathcal{H}_{M}^{\overline{\mu, \nu}} \underset{\operatorname{Bun}_{M}}{\times} \operatorname{Bun}_{B(M)}$ :

A point $\left(\left(x_{1}, \ldots, x_{N}\right), \mathcal{F}_{P}, \mathcal{F}_{M}^{\prime}, \beta_{M}, \mathcal{F}_{T}^{\prime}, \kappa_{M}^{\prime}\right)$ belongs to $Z^{\left\{\mu_{j}\right\},\left\{\nu_{j}\right\},\left\{\lambda_{j}\right\}}$ if for every $j$ and for every $\check{\lambda} \in \check{\Lambda}_{G}^{+}$, the composition

$$
\mathcal{L}_{\mathcal{F}_{T}^{\prime}}^{\check{\lambda}} \stackrel{\kappa_{M}^{\prime}}{\longrightarrow} U_{\mathcal{F}_{M}^{\prime}}^{\check{\lambda}} \stackrel{\beta_{M}}{\longrightarrow} U_{\mathcal{F}_{M}}^{\check{\lambda}}
$$

has a zero of order $\left\langle\lambda_{j}-\mu_{j}, \check{\lambda}\right\rangle$ at $x_{j}$.

It is clear that the preimage of $\overline{\operatorname{Bun}}_{B}^{\lambda}$ under

$$
\operatorname{Bun}_{P} \underset{\operatorname{Bun}_{M}}{\times} \mathcal{H}_{M}^{\overline{\mu, \nu}} \underset{\operatorname{Bun}_{M}}{\times} \operatorname{Bun}_{B(M)} \rightarrow \widetilde{\mu, \nu} \widetilde{\operatorname{Bun}}_{B, P} \stackrel{\mathfrak{k}_{P}}{\rightarrow} \overline{\operatorname{Bun}}_{B}
$$


is exactly the union of those $Z^{\left\{\mu_{j}\right\},\left\{\nu_{j}\right\},\left\{\lambda_{j}\right\}}$ 's for which $\Sigma \lambda_{j}=\lambda$.

Theorem 6.2.10 implies that the $*-$ restriction of $\mathcal{C}\left(\mathrm{IC}_{\widetilde{\operatorname{Bun}}_{P}}, \mathrm{IC}_{\overline{\mathrm{Bun}}_{B(M)}}\right)$ to $\frac{}{\mu, \nu} \mathrm{Bun}_{B, P}$ is smooth along the fibers of the projection $\overline{\mu, \nu} \operatorname{Bun}_{B, P} \rightarrow X^{\overline{\mu, \nu}}$. Therefore, to prove point (a) of the proposition, it suffices to show that for any $\left(x_{1}, \ldots, x_{N}\right) \in X^{N}-\Delta$, the codimension of $\left(\operatorname{Bun}_{P} \underset{\operatorname{Bun}_{M}}{\times} \Pi_{x_{j}} \mathcal{H}_{M}^{\nu_{j}} \underset{\operatorname{Bun}_{M}}{\times} \operatorname{Bun}_{B(M)}\right) \cap Z^{\left\{\mu_{j}\right\},\left\{\nu_{j}\right\},\left\{\lambda_{j}\right\}}$ inside the stack $\operatorname{Bun}_{P} \underset{\operatorname{Bun}_{M}}{\times \Pi_{x_{j}}} \mathcal{H}_{M}^{\nu_{j}} \underset{\operatorname{Bun}_{M}}{\times} \operatorname{Bun}_{B(M)}$ is $\geq\left\langle\lambda+|\overline{\mu, \nu}|, \check{\rho}_{M}\right\rangle$.

However, as in Sect. 3.3 .7 we obtain that the latter codimension equals

$$
\sum_{j} \operatorname{codim}\left(S_{M}^{\mu_{j}-\lambda_{j}} \cap \mathrm{Gr}_{M}^{-\mathrm{w}_{0}^{M}\left(\nu_{j}\right)}, \operatorname{Gr}_{M}^{-\mathrm{w}_{0}^{M}\left(\nu_{j}\right)}\right)
$$

(cf. Sect. 3.2.5) which is greater or equal than $\sum_{j}\left\langle\nu_{j}+\lambda_{j}-\mu_{j}, \check{\rho}_{M}\right\rangle=\left\langle\lambda+|\overline{\mu, \nu}|, \check{\rho}_{M}\right\rangle$.

Now let us prove point (b) of the proposition. To obtain the desired estimate on the dimension, it suffices to analyze the fibers of the map from $Z^{\left\{\mu_{j}\right\},\left\{\nu_{j}\right\},\left\{\lambda_{j}\right\}}$ to $\overline{\operatorname{Bun}}_{B}$. Note, that if $Z^{\left\{\mu_{j}\right\},\left\{\nu_{j}\right\},\left\{\lambda_{j}\right\}}$ is non-empty, then none of the $\lambda_{j}$ 's can be zero. Let us denote by $\bar{\lambda}$ the element of $\operatorname{Sym}^{\infty}\left(\Lambda_{G}^{\text {pos }}-0\right)$ corresponding to the collection $\lambda_{1}, \ldots, \lambda_{N}$.

We have a natural map of stacks:

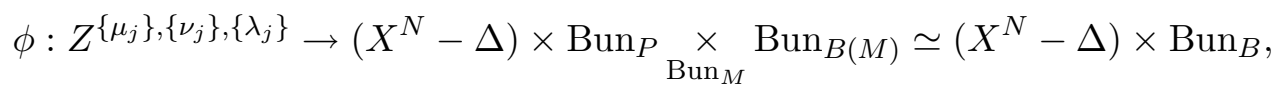

that sends

$$
\left(\left(x_{1}, \ldots, x_{N}\right), \mathcal{F}_{P}, \mathcal{F}_{M}^{\prime}, \beta_{M}, \mathcal{F}_{T}^{\prime}, \kappa_{M}^{\prime}\right) \mapsto\left(\left(x_{1}, \ldots, x_{N}\right), \mathcal{F}_{P}, \mathcal{F}_{T}^{\prime \prime}, \kappa_{M}^{\prime \prime}\right),
$$

where $\mathcal{F}_{T}^{\prime \prime}=\mathcal{F}_{T}^{\prime}\left(\Sigma\left(\lambda_{j}-\mu_{j}\right) \cdot x_{j}\right)$ and $\kappa_{M}^{\prime \prime}$ is the unique map that makes the square

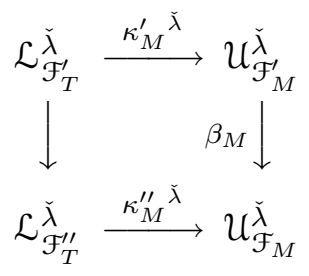

commute for each $\check{\lambda} \in \check{\Lambda}_{G}^{+}$. by

Now, the map $Z^{\left\{\mu_{j}\right\},\left\{\nu_{j}\right\},\left\{\lambda_{j}\right\}} \rightarrow \overline{\mathrm{Bun}}_{B}$ is a composition of the above map $\phi$ followed

$$
\left(X^{N}-\Delta\right) \times \operatorname{Bun}_{B} \rightarrow \overline{\operatorname{Bun}}_{B} \rightarrow X^{\bar{\lambda}} \times \operatorname{Bun}_{B} \stackrel{j_{\bar{\lambda}}}{\longrightarrow} \overline{\operatorname{Bun}}_{B}
$$

Since the map $\left(X^{N}-\Delta\right) \rightarrow X^{\bar{\lambda}}$ is étale and $j_{\bar{\lambda}}$ is a locally closed embedding, it suffices to analyze the fibers of $\phi$. However, as in Sect. 3.3.7, we obtain that they are isomorphic to $\Pi_{j}\left(\mathrm{Gr}_{M}^{\nu_{j}} \cap S_{M}^{\lambda_{j}-\mu_{j}}\right)$ and from Proposition 3.2.6 we obtain that their dimension is $\leq \sum_{j}\left\langle\nu_{j}+\lambda_{j}-\mu_{j}, \check{\rho}_{M}\right\rangle=\left\langle\lambda+|\overline{\mu, \nu}|, \check{\rho}_{M}\right\rangle$, which is what we had to prove.

\subsection{Composing Eisenstein series.}


7.2.1. At this point we are ready to prove the first part of Theorem 2.3.10, i.e. the existence of the isomorphism of functors: $\operatorname{Eis}_{T}^{G} \simeq \operatorname{Eis}_{M}^{G} \circ \operatorname{Eis}_{T}^{M}$ :

Proof. By definition, for $\mathcal{S} \in \operatorname{Sh}\left(\operatorname{Bun}_{T}\right)$,

$$
\operatorname{Eis}_{M}^{G} \circ \operatorname{Eis}_{T}^{M}(\mathcal{S}) \simeq \widetilde{\mathfrak{p}}_{P !}\left(\widetilde{\mathfrak{q}}_{P}^{*}\left(\overline{\mathfrak{p}}_{M !} \circ \overline{\mathfrak{q}}_{M}^{! *}(\mathcal{S})\right) \otimes \mathrm{IC}_{\widetilde{\operatorname{Bun}}_{P}}\right) \otimes\left(\overline{\mathbb{Q}_{\ell}}\left(\frac{1}{2}\right)[1]\right)^{\otimes-\operatorname{dim}\left(\operatorname{Bun}_{M}\right)},
$$

which, according to the projection formula, can be rewritten as

$$
\left(\widetilde{\mathfrak{p}}_{P} \circ \overline{\mathfrak{p}}_{M}^{\prime}\right)_{!}\left(\left(\overline{\mathfrak{q}}_{M} \circ \widetilde{\mathfrak{q}}_{P}^{\prime}\right)^{*}(\mathcal{S}) \otimes \mathcal{C}\left(\mathrm{IC}_{\widetilde{\operatorname{Bun}}_{P}}, \mathrm{IC}_{\overline{\overline{B u n}_{B(M)}}}\right)\right) \otimes\left(\overline{\mathbb{Q}_{\ell}}\left(\frac{1}{2}\right)[1]\right)^{\otimes-\operatorname{dim}\left(\operatorname{Bun}_{T}\right)} .
$$

However, the maps $\widetilde{\mathfrak{p}}_{P} \circ \overline{\mathfrak{p}}_{M}^{\prime}$ and $\overline{\mathfrak{p}} \circ \mathfrak{r}_{P}$ from $\widetilde{\operatorname{Bun}}_{B, P}$ to $\operatorname{Bun}_{G}$ coincide and so do the maps $\overline{\mathfrak{q}}_{M} \circ \widetilde{\mathfrak{q}}_{P}^{\prime}$ and $\overline{\mathfrak{q}} \circ \mathfrak{r}_{P}$ from $\widetilde{\operatorname{Bun}}_{B, P}$ to $\operatorname{Bun}_{T}$. Hence, (8) can be identified, using once again the projection formula, with

$$
\overline{\mathfrak{p}}_{!}\left(\overline{\mathfrak{q}}^{*}(\mathcal{S}) \otimes \mathfrak{r}_{P !}\left(\mathrm{C}\left(\mathrm{IC}_{\widetilde{\operatorname{Bun}}_{P}}, \mathrm{IC}_{\overline{\operatorname{Bun}}_{B(M)}}\right)\right)\right) \otimes\left(\overline{\mathbb{Q}_{\ell}}\left(\frac{1}{2}\right)[1]\right)^{\otimes-\operatorname{dim}\left(\operatorname{Bun}_{T}\right)},
$$

which, by Theorem 7.1.6 is the same as

$$
\overline{\mathfrak{p}}_{!}\left(\overline{\mathfrak{q}}^{*}(\mathcal{S}) \otimes \mathrm{IC}_{\overline{\overline{\operatorname{Bun}}_{B}}}\right) \otimes\left(\overline{\mathbb{Q}_{\ell}}\left(\frac{1}{2}\right)[1]\right)^{\otimes-\operatorname{dim}\left(\operatorname{Bun}_{T}\right)} \simeq \operatorname{Eis}_{T}^{G}(\mathcal{S}) .
$$

7.2.2. Now, we need to show that the constructed above isomorphism of functors $\operatorname{Eis}_{T}^{G} \simeq \operatorname{Eis}_{M}^{G} \circ \operatorname{Eis}_{T}^{M}$ is compatible with the action of Hecke functors. We will consider the local Hecke functors, i.e. ${ }_{x} H_{G},{ }_{x} H_{M}$ and ${ }_{x} H_{T}$, where $x$ is some fixed point of $X$.

Consider the fiber product

$$
\widetilde{\operatorname{Bun}}_{B, P}:={ }_{x, \infty} \widetilde{\operatorname{Bun}}_{P} \underset{\operatorname{Bun}_{M}}{\times} x, \infty \overline{\operatorname{Bun}}_{B(M)},
$$

and for $\mathcal{T}_{1} \in \operatorname{Sh}\left({ }_{x, \infty} \widetilde{\operatorname{Bun}}_{P}\right), \mathcal{T}_{2} \in \operatorname{Sh}\left({ }_{x, \infty} \overline{\operatorname{Bun}}_{B(M)}\right)$ we set

$$
\mathcal{C}\left(\mathcal{T}_{1}, \mathcal{T}_{2}\right):=\overline{\mathfrak{p}}_{M}^{\prime}\left(\mathcal{T}_{1}\right) \otimes \widetilde{\mathfrak{q}}_{P}^{\prime *}\left(\mathcal{T}_{2}\right) \otimes\left(\overline{\mathbb{Q}_{\ell}}\left(\frac{1}{2}\right)[1]\right)^{\otimes-\operatorname{dim}\left(\operatorname{Bun}_{M}\right)} \in \operatorname{Sh}\left({ }_{x, \infty} \widetilde{\operatorname{Bun}}_{B, P}\right) .
$$

Proposition 7.2.3. For $\mathcal{S} \in \mathrm{Sph}_{M}$ there is a functorial isomorphism

$$
\mathfrak{k}_{P !} \circ \mathcal{C}\left({ }_{x} H_{P, M}\left(\mathcal{S}, \mathcal{T}_{1}\right), \mathcal{T}_{2}\right) \simeq \mathfrak{k}_{P !} \circ \mathcal{C}\left(\mathcal{T}_{1},{ }_{x} H_{B(M), M}^{\leftarrow}\left(\mathcal{S}, \mathcal{T}_{2}\right)\right)
$$

Proof. Consider the fiber product

$$
{ }_{x, \infty} Z_{B, P}:={ }_{x, \infty} \widetilde{\operatorname{Bun}}_{P} \underset{\operatorname{Bun}_{M}}{\times} \mathcal{H}_{M} \underset{\operatorname{Bun}_{M}}{\times} x, \infty \overline{\operatorname{Bun}}_{B(M)} .
$$

It classifies the data of $\left(\mathcal{F}_{G}, \mathcal{F}_{M}, \widetilde{\kappa}_{P}, \mathcal{F}_{M}^{\prime}, \beta_{M}, \mathcal{F}_{T}^{\prime}, \kappa_{M}^{\prime}\right)$, with $\left(\mathcal{F}_{G}, \mathcal{F}_{M}, \widetilde{\kappa}_{P}\right) \in{ }_{x, \infty} \widetilde{\operatorname{Bun}}_{P}$, $\left(\mathcal{F}_{M}, \mathcal{F}_{M}^{\prime}, \beta_{M}\right) \in{ }_{x} \mathcal{H}_{M}$ and $\left(\mathcal{F}_{M}^{\prime}, \mathcal{F}_{T}^{\prime}, \kappa_{M}^{\prime}\right) \in{ }_{x, \infty} \overline{\operatorname{Bun}}_{B(M)}$. As in Sect. 4.1.2, there are two projections $h^{\leftarrow}$ and $h \rightarrow$ from this stack to ${ }_{x, \infty} \widetilde{\operatorname{Bun}}_{B, P}$ that "forget" $\mathcal{F}_{M}^{\prime}$ and $\mathcal{F}_{M}$, respectively.

By applying the projection formula, we obtain that for $\mathcal{S} \in \operatorname{Sph}_{M}, \mathcal{T}_{1} \in \operatorname{Sh}\left({ }_{x, \infty} \widetilde{\operatorname{Bun}}_{P}\right)$ and $\mathcal{T}_{2} \in \operatorname{Sh}\left({ }_{x, \infty} \overline{\operatorname{Bun}}_{B(M)}\right), \mathfrak{k}_{P !} \circ \mathcal{C}\left({ }_{x} H_{P, M}\left(\mathcal{S}, \mathcal{T}_{1}\right), \mathcal{T}_{2}\right)$ can be written as:

$$
\left(\mathfrak{k}_{P} \circ h^{\rightarrow}\right)_{!}\left(\left(\overline{\mathfrak{p}}_{M}^{\prime} \circ h^{\leftarrow}\right)^{*}\left(\mathcal{T}_{1}\right) \otimes\left(\widetilde{\mathfrak{q}}_{P}^{\prime} \circ h^{\rightarrow}\right)^{*}\left(\mathcal{T}_{2}\right) \otimes \mathcal{S}^{\prime}\right),
$$




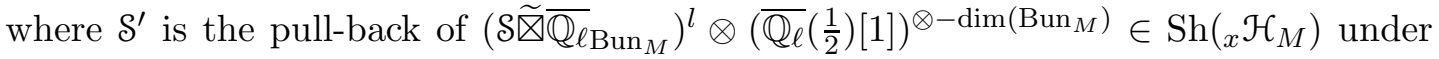
the map ${ }_{x, \infty} Z_{B, P} \rightarrow{ }_{x} \mathcal{H}_{M}$.

However, the maps $\mathfrak{k}_{P} \circ h^{\rightarrow}$ and $\mathfrak{k}_{P} \circ h^{\leftarrow}$ from ${ }_{x, \infty} Z_{B, P}$ to ${ }_{x, \infty} \overline{\operatorname{Bun}}_{B}$ coincide. Hence, (9) can be rewritten using the projection formula as

$$
\mathfrak{k}_{P !}\left(\overline{\mathfrak{p}}_{M}^{\prime}\left(\mathcal{T}_{1}\right) \otimes h_{!}^{\leftarrow}\left(\left(\widetilde{\mathfrak{q}}_{P}^{\prime} \circ h^{\rightarrow}\right)^{*}\left(\mathcal{T}_{2}\right) \otimes \mathcal{S}^{\prime}\right)\right)
$$

Moreover, it is easy to see that for any sheaf $\mathcal{T}^{\prime}$ on ${ }_{x, \infty} \widetilde{\operatorname{Bun}_{B, P}}$

$$
\left.\mathcal{T}^{\prime} \otimes h_{!}^{\leftarrow}\left(\left(\widetilde{\mathfrak{q}}_{P}^{\prime} \circ h^{\rightarrow}\right)^{*}\left(\mathcal{T}_{2}\right) \otimes \mathcal{S}^{\prime}\right) \simeq \mathcal{T}^{\prime} \otimes \widetilde{\mathfrak{q}}_{P}^{\prime}{ }^{*}{ }_{x} H_{B(M), M}^{\leftarrow}\left(\mathcal{S}, \mathcal{T}_{2}\right)\right) \otimes\left(\overline{\mathbb{Q}_{\ell}}\left(\frac{1}{2}\right)[1]\right)^{\otimes-\operatorname{dim}\left(\operatorname{Bun}_{M}\right)}
$$

and therefore, (9) can be identified with

$$
\left.\mathfrak{k}_{P !}\left(\overline{\mathfrak{p}}_{M}^{\prime}{ }^{*}\left(\mathcal{T}_{1}\right) \otimes \tilde{\mathfrak{q}}_{P}^{\prime}{ }^{*}{ }_{x} H_{B(M), M}^{\leftarrow}\left(\mathcal{S}, \mathcal{T}_{2}\right)\right)\right) \otimes\left(\overline{\mathbb{Q}}\left(\frac{1}{2}\right)[1]\right)^{\otimes-\operatorname{dim}\left(\operatorname{Bun}_{M}\right)}
$$

which is what we had to prove.

Observe now that we have the folowing natural isomorphisms of functors:

$$
\begin{aligned}
& \mathfrak{k}_{P} \circ \mathcal{C}\left({ }_{x} H_{P, G}^{?}\left(\mathcal{S}, \mathcal{T}_{1}\right), \mathcal{T}_{2}\right) \simeq{ }_{x} H_{B, G}^{?}\left(\mathcal{S}, \mathfrak{k}_{P !} \circ \mathcal{C}\left(\mathcal{T}_{1}, \mathcal{T}_{2}\right)\right), \mathcal{S} \in \operatorname{Sph}_{G} \text { and } \\
& \mathfrak{k}_{P} \circ \mathcal{C}\left(\mathcal{T}_{1},{ }_{x} H_{B(M), T}^{?}\left(\mathcal{S}^{\prime}, \mathcal{T}_{2}\right)\right) \simeq{ }_{x} H_{B, T}^{?}\left(\mathcal{S}^{\prime}, \mathfrak{k}_{P !} \circ \mathcal{C}\left(\mathcal{T}_{1}, \mathcal{T}_{2}\right)\right), \mathcal{S}^{\prime} \in \operatorname{Sph}_{T},
\end{aligned}
$$

where $?=$ either $\leftarrow$ or $\rightarrow$. It is easy to see that the above isomorphisms of functors are compatible in the following way:

Lemma 7.2.4. For $\mathcal{S} \in \operatorname{Sph}_{G}$ and $\mathcal{T}_{1}$ and $\mathcal{T}_{2}$ as above, the composition

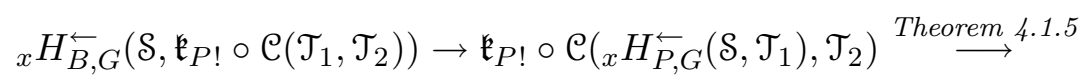

$$
\begin{aligned}
& \mathfrak{k}_{P !} \circ \mathrm{C}\left({ }_{x} H_{P, M}\left(\operatorname{gRes}_{M}^{G}(\mathcal{S}), \mathcal{T}_{1}\right), \mathcal{T}_{2}\right) \stackrel{\text { Proposition }}{\longrightarrow} \text { 7.2. } \mathfrak{k}_{P !} \circ \mathrm{C}\left(\mathcal{T}_{1},{ }_{x} H_{B(M), M}^{\leftarrow}\left(\operatorname{gRes}_{M}^{G}(\mathcal{S}), \mathcal{T}_{2}\right)\right)
\end{aligned}
$$

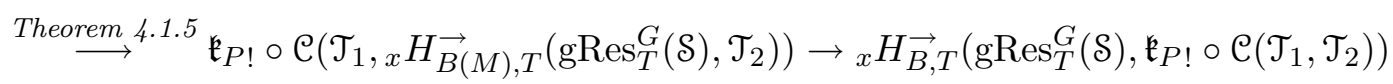

equals

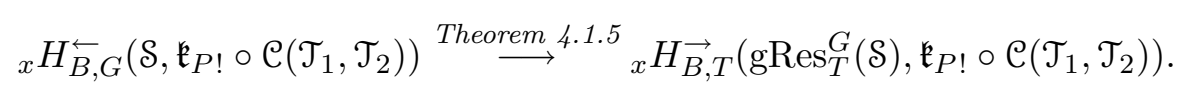

This readily implies what we need. Let $\mathcal{T}$ be an object of $\operatorname{Sh}\left(\operatorname{Bun}_{T}\right)$. Then, as in the proof of Theorem 2.3.7 given in Sect. 4.1.8, by taking $\mathcal{T}_{1}=\mathrm{IC}_{\overline{\operatorname{Bun}}_{P}}, \mathcal{T}_{2}=\mathrm{IC}_{\overline{\operatorname{Bun}}_{B(M)}}$, each of the two isomorphisms of functors of Lemma 7.2.4 produces an isomorphism ${ }_{x} H_{G}^{\leftarrow}\left(\mathcal{S}, \operatorname{Eis}_{T}^{G}(\mathcal{T})\right) \simeq \operatorname{Eis}_{T}^{G}\left({ }_{x} H_{T}^{\leftarrow}\left(\operatorname{gRes}_{T}^{G}(\mathcal{S}), \mathcal{T}\right)\right):$

For the latter, this will be the isomorphism of Theorem 2.1.5 and for the former, this will be the composition:

$$
\begin{aligned}
& { }_{x} H_{G}^{\leftarrow}\left(\mathcal{S}, \operatorname{Eis}_{T}^{G}(\mathcal{T})\right) \simeq{ }_{x} H_{G}^{\leftarrow}\left(\mathcal{S}, \operatorname{Eis}_{M}^{G} \circ \operatorname{Eis}_{T}^{M}(\mathcal{T})\right) \simeq \operatorname{Eis}_{M}^{G}\left({ }_{x} H_{M}^{\leftarrow}\left(\operatorname{gRes}_{M}^{G}(\mathcal{S}), \operatorname{Eis}_{T}^{M}(\mathcal{T})\right)\right) \simeq \\
& \operatorname{Eis}_{M}^{G} \circ \operatorname{Eis}_{T}^{M}\left({ }_{x} H_{T}^{\leftarrow}\left(\operatorname{Res}_{T}^{G}(\mathcal{S}), \mathcal{T}\right)\right) \simeq \operatorname{Eis}_{T}^{G}\left({ }_{x} H_{T}^{\leftarrow}\left(\operatorname{Res}_{T}^{G}(\mathcal{S}), \mathcal{T}\right)\right)
\end{aligned}
$$

Thus, the assertion of Lemma 7.2 .4 implies the second part of Theorem 2.3.10.

\subsection{Computation in rank 1.}


7.3.1. From this moment on we will be concerned with the proof of Theorem 2.1.8. Observe that Theorem 2.3.10 reduces the assertion of Theorem 2.1.8 to the case when $G$ is of semi-simple rank 1:

Indeed, by induction we may suppose that our element $\mathrm{w}$ is a simple reflection $s_{\mathfrak{i}}$ corresponding to $\mathfrak{i} \in \mathcal{J}$, and according to Theorem 2.3.10 it is enough to construct an isomorphism $\operatorname{Eis}_{T}^{M_{\mathfrak{i}}}\left(s_{\mathfrak{i}} \cdot \mathcal{S}\right) \simeq \operatorname{Eis}_{T}^{M_{\mathfrak{i}}}(\mathcal{S})$, where $M_{\mathfrak{i}}$ is the corresponding sub-minimal Levi, i.e. $\mathcal{J}_{M_{\mathrm{i}}}=\{\mathfrak{i}\}$.

Henceforth, $G$ will be a reductive group of semi-simple rank 1 . The (only) positive root, fundamental weight and the non-trivial Weyl group elements will be denoted by $\check{\alpha}$, $\check{\omega}$ and $\sigma$, respectively. Note that the Weyl module $\mathcal{V}^{\breve{\omega}}$ is 2-dimensional and irreducible.

Recall that the isomorphism $\operatorname{Eis}(\sigma \cdot \mathcal{S}) \stackrel{f \cdot e q}{\longrightarrow} \operatorname{Eis}(\mathcal{S})$ (for $\mathcal{S} \in \operatorname{Sh}\left(\operatorname{Bun}_{T}\right)^{r e g}$ ) should depend on a specific choice of a lift of $\sigma$ to $N(\check{T})$. The isomorphism that we will construct corresponds to the image of $\left(\begin{array}{cc}0 & 1 \\ -1 & 0\end{array}\right) \in S L(2)$ under the canonical map $S L(2) \rightarrow \check{G}$. If we multiply this element on the left by $\tau \in \check{T}$, the corresponding isomorphism must be multiplied by $\mu(\tau)$ for $\mathcal{S}$, which is concentrated on $\operatorname{Bun}_{T}^{\mu}$.

7.3.2. Consider the fiber product $\operatorname{Bun}_{G, T}:=\operatorname{Bun}_{G} \underset{\operatorname{Bun}_{G /[G, G]}}{\times} \operatorname{Bun}_{T}$. We will denote by $p_{G}$ and $p_{T}$ the projections from $\operatorname{Bun}_{G, T}$ to $\operatorname{Bun}_{G}$ and $\operatorname{Bun}_{T}$, respectively. The starting point of our discussion is the following observation:

Proposition 7.3.3. For $G$ as above, the stack $\overline{\mathrm{Bun}}_{B}$ classifies triples $\left(\mathcal{F}_{G}, \mathcal{F}_{T}, \kappa\right)$, where $\left(\mathcal{F}_{G}, \mathcal{F}_{T}\right) \in \operatorname{Bun}_{G, T}$ and $\kappa$ is an injective (=non-zero) map $\mathcal{L}_{\mathcal{F}_{T}}^{\breve{\omega}} \rightarrow \mathcal{V}_{\mathcal{F}_{G}}^{\breve{\omega}}$. In particular, $\overline{\mathrm{Bun}}_{B}$ is smooth.

Proof. Since $\check{\omega}$ is the only fundamental weight, $\overline{\mathrm{Bun}}_{B}$ is a evidently a closed substack in the stack classifying triples $\left(\mathcal{F}_{G}, \mathcal{F}_{T}, \kappa\right)$ as above. To show that this closed embedding is an isomorphism, one has to show that any map $\kappa$ satisfies the Plücker relations, which is evident.

To show that $\overline{\mathrm{Bun}}_{B}$ is smooth we argue as follows: consider the algebraic stack $C o h_{1}$ which classifies coherent sheaves on $X$ of generic rank 1. It is known (cf. [19]) that $\mathrm{Coh}_{1}$ is smooth.

We have a natural map $\overline{\operatorname{Bun}}_{B} \rightarrow C o h_{1}$, which sends a triple $\left(\mathcal{F}_{G}, \mathcal{F}_{T}, \kappa\right)$ as above to the quotient $\nu^{\check{\omega}} / \operatorname{Im}(\kappa)$. It is easy to see that this map is smooth, hence $\overline{\operatorname{Bun}}_{B}$ is smooth too.

Now, let $\overline{\operatorname{Bun}}_{B}^{r}$ be the stack that classifies triples $\left(\mathcal{F}_{G}, \mathcal{F}_{T}, \kappa\right)$, where $\left(\mathcal{F}_{G}, \mathcal{F}_{T}\right) \in$ $\operatorname{Bun}_{G, T}$ as before, but $\kappa$ is allowed to be an arbitrary map $\mathcal{L}_{\mathcal{F}_{T}}^{\breve{\omega}} \rightarrow \mathcal{V}_{\mathcal{F}_{G}}^{\breve{\omega}}$. We will denote the natural projections from $\overline{\operatorname{Bun}}_{B}^{r}$ to $\operatorname{Bun}_{G}$ and $\operatorname{Bun}_{T}$ by $\overline{\mathfrak{p}}^{r}$ and $\overline{\mathfrak{q}}^{r}$, respectively.

By construction, $\overline{\mathrm{Bun}}_{B}$ is an open substack in $\overline{\mathrm{Bun}}_{B}^{r}$, which corresponds to non-zero $\kappa$ 's. The complement, i.e. the locus $\kappa=0$ projects isomorphically onto $\operatorname{Bun}_{G, T}$.

For $\mathcal{S} \in \operatorname{Sh}\left(\operatorname{Bun}_{T}\right)$, we define its renormalized Eisenstein series as

$$
\operatorname{Eis}^{r}(\mathcal{S}):=\overline{\mathfrak{p}}_{!}^{r} \circ \overline{\mathfrak{q}}^{r *}(\mathcal{S}) \otimes\left(\overline{\mathbb{Q}_{\ell}}\left(\frac{1}{2}\right)[1]\right)^{\otimes ?},
$$


where ? equals $g-1+\langle\mu, \check{\rho}\rangle$ over the $\mu$-th connected component of $\operatorname{Bun}_{T}$.

Note that the map $\overline{\mathfrak{p}}^{r}$ is non-representable, since Bun$T$ is a stack and not a scheme. Therefore, $\operatorname{Eis}^{r}$ takes values not in $\operatorname{Sh}\left(\operatorname{Bun}_{G}\right)$, but rather in the corresponding "unbounded on the left" derived category.

Proposition 7.3.4. For $\mathcal{S} \in \mathrm{Sh}\left(\mathrm{Bun}_{T}\right)^{r e g}$, there is a canonical isomorphism $\operatorname{Eis}(\mathcal{S}) \simeq$ $\operatorname{Eis}^{r}(\mathcal{S})$.

Proof. By construction, we have a natural map $\operatorname{Eis}(\mathcal{S}) \rightarrow \operatorname{Eis}^{r}(\mathcal{S})$, which corresponds to the open embedding $\overline{\operatorname{Bun}}_{B} \hookrightarrow \overline{\operatorname{Bun}}_{B}^{r}$. Its cone is by definition $p_{G !} \circ p_{T}^{*}(\mathcal{S})$. However,the latter complex is the same as the pull-back under $\operatorname{Bun}_{G} \rightarrow \operatorname{Bun}_{G /[G, G]}$ of $\mathfrak{f}_{!}(\mathcal{S}) \otimes\left(\overline{\mathbb{Q}_{\ell}}\left(\frac{1}{2}\right)[1]\right)^{\otimes}$ ? (cf. Sect. 2.1.7). This implies our assertion.

Therefore, in order to construct an isomorphism $\operatorname{Eis}(\sigma \cdot \mathcal{S}) \stackrel{f . e q}{\longrightarrow} \operatorname{Eis}(\mathcal{S})$ for an object $\mathcal{S} \in \mathrm{Sh}\left(\operatorname{Bun}_{T}\right)^{r e g}$, it suffuces to construct a functorial isomorphism

$$
\operatorname{Eis}^{r}(\sigma \cdot \mathcal{S}) \rightarrow \operatorname{Eis}^{r}(\mathcal{S}), \forall \mathcal{S} \in \operatorname{Sh}\left(\operatorname{Bun}_{T}\right) .
$$

This will be done in the following general set-up:

7.3.5. Let $y$ be a stack and let $\varepsilon_{0}$ and $\varepsilon_{1}$ be two locally free coherent sheaves on $y$ and let $f: \mathcal{E}_{0} \rightarrow \mathcal{E}_{1}$ be a map between them; we will regard $\mathcal{E}_{0} \rightarrow \mathcal{E}_{1}$ as a length-2 complex on $y$; let $\chi(\mathcal{E})$ be its Euler characteristic, i.e. $\chi(\mathcal{E})=\operatorname{rk}\left(\mathcal{E}_{0}\right)-\operatorname{rk}\left(\mathcal{E}_{1}\right)$. We will denote by $\mathcal{E}^{*}$ the dual complex, in other words, $\mathcal{E}^{*}$ corresponds to the adjoint map $\mathcal{E}_{1}^{*} \stackrel{f^{*}}{\rightarrow} \mathcal{E}_{0}^{*}$.

Let $K_{\mathcal{E}}$ denote the following object of $\operatorname{Sh}(y)$ : we can regard $\mathcal{E}_{0}$ and $\mathcal{E}_{1}$ as groupschemes over $y$, and let $h^{0}(\varepsilon) \subset \mathcal{E}_{0}$ denote the group-scheme-theoretic kernel of $f$. Then $K_{\mathcal{E}}$ is the !-direct image of $\overline{\mathbb{Q}_{\ell}} h^{0}(\mathcal{E})$ on $y$ tensored by $\left(\overline{\mathbb{Q}_{\ell}}\left(\frac{1}{2}\right)[1]\right)^{\otimes \chi(\mathcal{E})}$.

Lemma 7.3.6. Under the above circumstances, there is a canonical isomorphism $K_{\mathcal{E}} \stackrel{e q_{\varepsilon}}{\longrightarrow} K_{\mathcal{E} *}$.

Proof. Indeed, 9 let us consider the fiber product $\mathcal{E}_{0} \underset{y}{\times} \mathcal{E}_{1}^{*}$. We have the evaluatuion map $\mathcal{E}_{0} \underset{y}{\times} \mathcal{E}_{1}^{*} \rightarrow \mathbb{A}^{1}$ and let us denote by $\Psi$ the pull-back of the Artin-Schreier sheaf on $\mathbb{A}^{1}$ under this map, tensored by $\left(\overline{\mathbb{Q}_{\ell}}\left(\frac{1}{2}\right)[1]\right)^{\mathrm{rk}\left(\varepsilon_{0}\right)} \otimes\left(\overline{\mathbb{Q}_{\ell}}\left(\frac{1}{2}\right)[1]\right)^{\mathrm{rk}\left(\mathcal{E}_{1}^{*}\right)}$.

To construct the isomorphism $e q_{\mathcal{E}}$, it is enough to show that both $K_{\mathcal{E}}$ and $K_{\mathcal{E}^{*}}$ can be canonically identified with the !-direct image of $\Psi$ under the projection $\mathcal{E}_{0} \underset{y}{\times} \mathcal{E}_{1}^{*} \rightarrow y$.

However, the !-direct image of $\Psi$ under the projection $\mathcal{E}_{0} \underset{y}{\times} \mathcal{E}_{1}^{*} \rightarrow \mathcal{E}_{0}$ is evidently isomorphic to $\left(\overline{\mathbb{Q}_{\ell}}\left(\frac{1}{2}\right)[1]\right)_{h^{0}(\mathcal{E})}^{\otimes \chi(\mathcal{E})}$ and hence is further push-forward onto $y$ can be identified with $K_{\mathcal{E}}$. The assertion for $K_{\mathcal{E}^{*}}$ follows by symmetry.

The following properties of the isomorphism $e q_{\mathcal{E}}$ follow from the construction:

\footnotetext{
${ }^{6}$ The construction described below is due to V. Drinfeld.
} 
Lemma 7.3.7. (a) The composition $e q_{\mathcal{E}^{*}} \circ e q_{\mathcal{E}}: K_{\mathcal{E}} \rightarrow K_{\mathcal{E}}$ is the identity map.

(b) Let $f^{\prime}: \mathcal{E}_{0}^{\prime} \rightarrow \mathcal{E}_{1}^{\prime}$ be another 2-complex consisting of locally free sheaves and let $\left(\mathcal{E}_{0} \stackrel{f}{\rightarrow} \mathcal{E}_{1}\right) \rightarrow\left(\mathcal{E}_{0}^{\prime} \stackrel{f^{\prime}}{\rightarrow} \mathcal{E}_{1}^{\prime}\right)$ be a quasi-isomorphism. Then there are canonical isomorphisms $h^{0}(\mathcal{E}) \simeq h^{0}\left(\mathcal{E}^{\prime}\right)$ and $h^{0}\left(\mathcal{E}^{*}\right) \simeq h^{0}\left(\mathcal{E}^{\prime *}\right)$ and $e q_{\mathcal{E}}$ coincides with eq $\mathcal{E}^{\prime}$ up to $(-1)^{\mathrm{rk}\left(\mathcal{E}_{1}\right)-\mathrm{rk}\left(\mathcal{E}_{1}^{\prime}\right)}$.

7.3.8. We will apply the above discussion to $y=\operatorname{Bun}_{G, T}$. First, let us consider the 2-dimensional vector bundle $\mathcal{M}$ over $X \times \operatorname{Bun}_{G, T}$, whose fiber over $\left(\mathcal{F}_{G}, \mathcal{F}_{T}\right) \in \operatorname{Bun}_{G, T}$ is $\underline{\operatorname{Hom}}\left(\mathcal{L}_{\mathcal{F}_{T}}^{\breve{\omega}}, \mathcal{V}_{\mathcal{F}_{G}}^{\breve{\omega}}\right)$. Set $\mathcal{E}$ to be the derived direct image of $\mathcal{M}$ with respect to the projection $X \times \operatorname{Bun}_{G, T} \rightarrow \operatorname{Bun}_{G, T}$.

It is clear that locally on $\operatorname{Bun}_{G, T}$, we can $\mathcal{E}$ represent by a 2 -complex $\mathcal{E}_{0} \rightarrow \mathcal{E}_{1}$ of vector bundles with a fixed parity of $\operatorname{rk}\left(\mathcal{E}_{1}\right)$ : Indeed, let $y \in X$ be an arbitrary point and let $n$ be a large postive integer. Let $S_{y, n}$ be an open substack of Bun $\operatorname{Bu}_{G, T}$ which corresponds to those $\left(\mathcal{F}_{G}, \mathcal{F}_{T}\right)$ for which $\operatorname{Ext}^{1}\left(\mathcal{L}_{\mathcal{F}_{T}}^{\check{\omega}}, \mathcal{V}_{\mathcal{F}_{G}}^{\check{\omega}}(n \cdot y)\right)=0$.

Then over a point $\left(\mathcal{F}_{G}, \mathcal{F}_{T}\right) \in S_{y, n}$ we set

$$
\mathcal{E}_{0}=\operatorname{Hom}\left(\mathcal{L}_{\mathcal{F}_{T}}^{\check{\omega}}, \mathcal{V}_{\mathcal{F}_{G}}^{\check{\omega}}(n \cdot y)\right) \text { and } \mathcal{E}_{1}=\operatorname{Hom}\left(\mathcal{L}_{\mathcal{F}_{T}}^{\check{\omega}}, \mathcal{V}_{\mathcal{F}_{G}}^{\check{\omega}}(n \cdot y) / \mathcal{V}_{\mathcal{F}_{G}}^{\check{\omega}}\right) .
$$

Thus, we obtained a correctly defined object $K_{\mathcal{E}} \in \operatorname{Sh}\left(\operatorname{Bun}_{G, T}\right)$. It is easy to see that $h^{0}(\mathcal{E}) \simeq \overline{\operatorname{Bun}}_{B}^{r}$ and hence $\mathcal{K}_{E}$ is the kernel of the renormalized Eisenstein series functor $\operatorname{Eis}^{r}$, i.e.

$$
\operatorname{Eis}^{r}(S) \simeq p_{G !}\left(p_{T}^{*}(\mathcal{S}) \otimes \mathcal{K}_{E}\right) .
$$

Consider now the action of the involution $\sigma \cdot$ on $\operatorname{Bun}_{G, T}$. We obtain from Lemma 7.3.6 and Lemma 7.3.7 (b) that in order to construct the isomorphism of (10), it suffices to show that $\sigma \cdot \mathcal{K}_{\mathcal{E}} \simeq \mathcal{K}_{\mathcal{E}^{*}}$. For that, by Serre's duality, it suffices to show that over $X \times \operatorname{Bun}_{G, T}$

$$
\sigma \cdot \mathcal{M} \simeq \mathcal{M}^{*} \otimes\left(\Omega_{X} \otimes \mathcal{O}_{\mathrm{Bun}_{G, T}}\right)
$$

where $\mathcal{M}^{*}$ is the dual of $\mathcal{M}$.

By definition, the fiber of $\sigma \cdot \mathcal{M}$ over $\left(\mathcal{F}_{G}, \mathcal{F}_{T}\right) \in \operatorname{Bun}_{G, T}$ is

$$
\operatorname{Hom}\left(\mathcal{L}_{\mathcal{F}_{T}}^{\check{\omega}-\check{\alpha}} \otimes \Omega_{X}^{-1}, \mathcal{V}_{\mathcal{F}_{G}}^{\check{\omega}}\right) \simeq \operatorname{Hom}\left(\mathcal{L}_{\mathcal{F}_{T}}^{-\check{\omega}}, \mathcal{L}_{\mathcal{F}_{T}}^{-2 \check{\omega}+\check{\alpha}} \otimes \mathcal{V}_{\mathcal{F}_{G}} \otimes \Omega_{X}\right)
$$

However, since $\operatorname{dim}\left(\mathcal{V}^{\check{\omega}}\right)=2, \mathcal{V}_{\mathcal{F}_{G}}^{\check{\omega}} \simeq\left(\mathcal{V}_{\mathcal{F}_{G}}^{\check{\omega}}\right)^{*} \otimes \operatorname{det}\left(\mathcal{V}_{\mathcal{F}_{G}}^{\check{\omega}}\right) \simeq\left(\mathcal{V}_{\mathcal{F}_{G}}^{\check{\omega}}\right)^{*} \otimes \mathcal{L}_{\mathcal{F}_{T}}^{2 \check{\omega}-\check{\alpha}}$.

Therefore, the above fiber of $\sigma \cdot \mathcal{M}$ can be identified with $\operatorname{Hom}\left(\left(\mathcal{L}_{\mathcal{F}_{T}}^{\check{\omega}}\right)^{*},\left(\mathcal{V}_{\mathcal{F}_{G}}^{\check{\omega}}\right)^{*} \otimes \Omega_{X}\right)$, which is what we had to show.

7.3.9. The above construction yields an isomorphism of functors $\operatorname{Eis}(\sigma \cdot \mathcal{S}) \stackrel{f \cdot e q^{\prime}}{\longrightarrow} \operatorname{Eis}(\mathcal{S})$. Our task now is to modify it in such a way that it becomes compatible with the isomorphism of Theorem 2.1.5 in the sense of Sect. 2.1.9 for the specified above choice of a lifting of $\sigma$ to $N(\check{T})$.

Remark. There is another way to obtain the functional equation isomorphism, using the geometric analog of the Whittaker model (cf. [14]). By comparing the two, one can show that the isomorphism $f . e q^{\prime}$ that we constructed corresponds to the element $\left(\begin{array}{cc}0 & 1 \\ -1 & 0\end{array}\right) \cdot \epsilon \in S L(2)$, where $\epsilon= \pm 1$, depending on the genus of $X$. 
Let us first consider the case of $G=G L(2)$, in which case the Langlands dual group is $G L(2)$ as well. For two integers $d_{1}, d_{2}$, we will denote by $\left(d_{1}, d_{2}\right)$ the corresponding coweight of $G L(2)$.

Proposition 7.3.10. For $\mathcal{S} \in \operatorname{Sh}\left(\operatorname{Bun}_{T}^{\mu}\right)$ with $\mu=\left(d_{1}, d_{2}\right)$, there is a functorial isomorphism $H_{G}^{(1,0)}\left(\operatorname{Eis}^{r}(\mathcal{S})\right) \simeq \operatorname{Eis}^{r}\left(H_{T}^{(1,0)}(\mathcal{S})\right) \oplus \operatorname{Eis}^{r}\left(H_{T}^{(0,1)}(\mathcal{S})\right)$, such that:

(a) For $\mathcal{S} \in \mathrm{Sh}\left(\mathrm{Bun}_{T}\right)^{r e g}$, it coincides with the isomorphism of Theorem 2.1.5.

(b) The square

$$
\begin{aligned}
{ }_{x} H_{G}^{(1,0)}\left(\operatorname{Eis}^{r}(\sigma \cdot \mathcal{S})\right) & \longrightarrow \operatorname{Eis}^{r}\left(\sigma \cdot\left({ }_{x} H_{T}^{(1,0)}(\mathcal{S})\right)\right) \oplus \operatorname{Eis}^{r}\left(\sigma \cdot\left({ }_{x} H_{T}^{(0,1)}(\mathcal{S})\right)\right) \\
f . e q^{\prime} \downarrow & -f \cdot e q^{\prime} \cdot \epsilon\left(d_{1}, d_{2}\right) \oplus f \cdot e q^{\prime} \cdot \epsilon\left(d_{1}, d_{2}\right) \downarrow \\
{ }_{x} H_{G}^{(1,0)}\left(\operatorname{Eis}^{r}(\mathcal{S})\right) & \longrightarrow \operatorname{Eis}^{r}\left({ }_{x} H_{T}^{(1,0)}(\mathcal{S})\right) \oplus \operatorname{Eis}^{r}\left({ }_{x} H_{T}^{(0,1)}(\mathcal{S})\right)
\end{aligned}
$$

commutes, where $\epsilon\left(d_{1}, d_{2}\right)= \pm 1$. Moreover, $\epsilon\left(d_{1}+d, d_{2}+d\right)=\epsilon\left(d_{1}, d_{2}\right)$.

Proof. Consider the fiber product $Z:={ }_{x} \mathcal{H}_{G}^{(1,0)} \underset{\operatorname{Bun}_{G}}{\times \overline{\operatorname{Bun}}_{B}^{r}}$, where ${ }_{x} \mathcal{H}_{G}^{(1,0)} \rightarrow \operatorname{Bun}_{G}$ is the map $h_{G}$. By definition, this stack classifies quintuples $\left(\mathcal{M}, \mathcal{M}^{\prime}, \mathcal{L}^{\prime}, \kappa^{\prime}, \beta\right)$, where $\mathcal{M}$, $\mathcal{M}^{\prime}$ are rank 2-bundles, $\beta: \mathcal{M}^{\prime} \hookrightarrow \mathcal{M}$ is an embedding of coherent sheaves such that $\mathcal{M} / \mathcal{M}^{\prime}$ is a skyscraper sheaf at $x, \mathcal{L}^{\prime}$ is a line bundle and $\kappa^{\prime}: \mathcal{L}^{\prime} \rightarrow \mathcal{M}^{\prime}$ is an arbitrary map.

We have a natural map $\phi: Z \rightarrow \overline{\operatorname{Bun}}_{B}^{r}$, which sends a quintuple $\left(\mathcal{M}, \mathcal{M}^{\prime}, \mathcal{L}^{\prime}, \kappa^{\prime}, \beta\right)$ as above to $(\mathcal{M}, \mathcal{L}, \kappa)$, where $\mathcal{L}=\mathcal{L}^{\prime}$ and $\kappa$ is the composition

$$
\mathcal{L}=\mathcal{L}^{\prime} \rightarrow \mathcal{M}^{\prime} \rightarrow \mathcal{M}
$$

Let ${ }_{x, \geq(1,-1)} \overline{\operatorname{Bun}}_{B}^{r} \subset \overline{\operatorname{Bun}}_{B}^{r}$ we the closed substack of $\overline{\operatorname{Bun}}_{B}^{r}$, which corresponds to triples $(\mathcal{M}, \mathcal{L}, \kappa)$ such that $\kappa: \mathcal{L} \rightarrow \mathcal{M}(-x)$. To prove point (a) of the proposition, it is enough to show that

$$
\phi_{!}\left(\overline{\mathbb{Q}_{\ell} Z}\right) \simeq \overline{\mathbb{Q}_{\ell}} \overline{\operatorname{Bun}}_{B}^{r} \oplus \overline{\mathbb{Q}}_{x, \geq(1,-1)} \overline{\operatorname{Bun}}_{B}^{r}(-1)[-2] .
$$

Remark. If we knew that $\overline{\mathbb{Q}_{\ell}} \overline{\operatorname{Bun}}_{B}^{r}$ is $\mathrm{IC}_{\overline{\mathrm{Bun}}_{B}^{r}}^{r}$ up to cohomological shift and Tate's twist, the last asserion would be obvious from the decomposition theorem (cf. below). Since the latter fact is not in general true, we have to proceed differently.

Take $y \neq x$ and let $S_{y, n} \subset \operatorname{Bun}_{G, T}$ be as above. Let $f: \mathcal{E}_{0} \rightarrow \mathcal{E}_{1}$ be as above as well, and let $\delta_{\mathcal{E}_{1}}$ be the constant sheaf on the 0 -section of $\mathcal{E}_{1}$. Consider $Z_{\mathcal{E}_{0}}:={ }_{x} \mathcal{H}_{G}^{(1,0)} \underset{\operatorname{Bun}_{G}}{\times} \mathcal{E}_{0}$; as above, we have a natural map $\phi_{\mathcal{E}_{0}}: Z_{\mathcal{E}_{0}} \rightarrow \mathcal{E}_{0}$. Let ${ }_{x, \geq(1,-1)} \mathcal{E}_{0} \subset \mathcal{E}_{0}$ be the closed substack that classifies triples $(\mathcal{L}, \mathcal{M}, \mathcal{L} \rightarrow \mathcal{M}(n \cdot y))$, for which $\operatorname{Im}(\mathcal{L}) \subset \mathcal{M}(n \cdot y-x)$.

It is clear that over the open subset $\mathcal{E}_{0}-{ }_{x, \geq(1,-1)} \mathcal{E}_{0}$, the map $\phi_{\mathcal{E}_{0}}$ is an isomorphism and over ${ }_{x, \geq(1,-1)} \mathcal{E}_{0}$ this is a fibration with the typical fiber $\mathbb{P}^{1}$. Since $\mathcal{E}_{0}$ is smooth, from the decomposition theorem we obtain that

$$
\phi_{\mathcal{E}_{0} !}\left(\overline{\mathbb{Q}}_{\ell Z_{\varepsilon_{0}}}\right) \simeq{\overline{\mathbb{Q}} \ell \varepsilon_{0}} \oplus \overline{\mathbb{Q}}_{\ell_{x, \geq(1,-1)} \varepsilon_{0}}(-1)[-2] .
$$

By construction, $\overline{\mathbb{Q}_{\ell}} \overline{\operatorname{Bun}}_{B}^{r} \simeq \overline{\mathbb{Q}_{\ell} \varepsilon_{0}} \otimes f^{*}\left(\delta_{\mathcal{E}_{1}}\right)$. Moreover, since $x \neq y, \mathcal{E}_{1}$ does not "distinguish" between $\mathcal{M}$ and $\mathcal{M}^{\prime}$ and we obatin:

$$
\phi_{!}\left(\overline{\mathbb{Q}_{\ell} Z}\right) \simeq \phi_{\mathcal{E}_{0} !}\left({\overline{\mathbb{Q}} \ell Z_{\varepsilon_{0}}}\right) \otimes f^{*}\left(\delta_{\mathcal{E}_{1}}\right) .
$$


Therefore,

$$
\begin{aligned}
& \phi_{!}\left(\overline{\mathbb{Q}}_{\ell Z}\right) \simeq \overline{\mathbb{Q}}_{\ell} \varepsilon_{0} \otimes f^{*}\left(\delta_{\mathcal{E}_{1}}\right) \oplus \overline{\mathbb{Q}}_{\ell_{x, \geq(1,-1)}} \varepsilon_{0}(-1)[-2] \otimes f^{*}\left(\delta_{\mathcal{E}_{1}}\right) \simeq \\
& \overline{\mathbb{Q}}_{\ell} \overline{\operatorname{Bun}}_{B}^{r} \oplus \overline{\mathbb{Q}}_{\ell_{x, \geq(1,-1)}} \overline{\operatorname{Bun}}_{B}^{r}(-1)[-2],
\end{aligned}
$$

which is what we had to prove.

The finishes the proof of point (a) of the proposition. Point (b) is a tedious but straightforward verification, which we omit.

Now, for our group $G$ let $\mu \mapsto d(\mu)$ be the map $\Lambda \rightarrow \Lambda_{G / Z(G)} \simeq \mathbb{Z}$. Let us choose a function $\epsilon^{\prime}: \mathbb{Z} \rightarrow \pm 1$, so that $\epsilon^{\prime}(d+1) \cdot \epsilon^{\prime}(d)=\epsilon(d, 0)$, where $\epsilon$ is as in the above proposition. We set the functional equation isomorphism $\operatorname{Eis}(\sigma \cdot \mathcal{S}) \stackrel{f . e q}{\longrightarrow} \operatorname{Eis}(\mathcal{S})$ to be $f . e q^{\prime} \cdot \epsilon^{\prime}(d(\mu))$ for $\mathcal{S} \in \operatorname{Sh}\left(\operatorname{Bun}_{T}^{\mu}\right)$. Let us show that it satifies the required compatibility condition with respect to the isomorphism of Theorem 2.1.5.

First, let us observe that if $G_{1} \rightarrow G_{2}$ is an embedding of groups whose cokernel is a torus, then the statement that we have to check for $G_{1}$ follows from that for $G_{2}$. It is easy to see that any $G$ with $[G, G] \simeq S L(2)$ can be embedded into a product of $G L(2)$ and a torus. This allows to replace our initial $G$ by $G L(2)$.

For $G L(2)$ every irreducible module $V$ over the Langlands dual group can be embedded into a tensor power of the tautologiocal representation $V^{(1,0)}$ times a 1-dimensional representation. Now, since the isomorphism of Theorem 2.1.5 is compatible with tensor products of $\breve{G}$-representaions and is evident for 1-dimensional representations, it

suffices to treat the case of the Hecke functor $H_{G L(2)}^{(1,0)}$. In the latter case the assertion follows from Lemma 7.3.10.

\section{APPENDiX}

In this Appendix we will prove Proposition 2.2.9. The proof presented below is a variant of an argument that was explained to us by G. Prasad.

Let $\check{G}$ be a connected reductive algebraic group over an algebraically closed field of characteristic 0 and let $\check{T}$ be a Cartan subgroup of $\check{G}$. Denote by $N(\check{T})$ the normalizer of $\check{T}$ in $\check{G}$. Suppose that we are given a closed subgroup $\Gamma \subset \check{T}$ and and element $F \in \check{G}$ such that $F$ normalizes $\Gamma$. Let $\widetilde{\Gamma}$ be the minimal closed subgroup of $\check{G}$ which contains $\Gamma$ and $F$.

Theorem A.1. Assume that $\widetilde{\Gamma}$ is irreducible, i.e. there is no proper parabolic subgroup $\check{P}$ in $\check{G}$ such that $\widetilde{\Gamma} \subset \check{P}$. Then

(a) For every root $\check{\alpha}: \check{T} \rightarrow \mathbb{G}_{m}$, the restriction of $\check{\alpha}$ to $\Gamma$ is not identically equal to 1 .

(b) F normalizes $\check{T}$.

Proposition A.2. Let $Z$ denote the centralizer of $\Gamma$ and let $Z^{0}$ denote the connected component of the identity in $Z$. Then $Z^{0}$ is commutative.

Let us show first that the above proposition imples the Theorem A.1. 
Proof. Assume that (a) does not hold, i.e. that there exists a root $\check{\alpha}$ of $\check{G}$ such that $\left.\check{\alpha}\right|_{\Gamma}=1$. Let $p_{\check{\alpha}}: S L(2) \rightarrow \check{G}$ denote the corresponding map. Then it is easy to see that $\Gamma$ commutes with the image of $p_{\check{\alpha}}$. Hence $Z^{0}$ contains $\operatorname{Im}\left(p_{\check{\alpha}}\right)$ and therefore it is not commutative.

To prove (b) let us note that $\check{T} \subset Z^{0}$. Hence, if $Z^{0}$ is commutative, it follows that $Z^{0}=\check{T}$. On the other hand, $F$ clearly normalizes $Z^{0}$. Hence $F \in N(\check{T})$.

Now let us prove Proposition A.2. The proof will use the following well-known lemma:

Lemma A.3. Let $H$ be an algebraic group (over an algebraically closed field of characteristic zero) and let $\sigma$ be an automorphism of $H$. Then there exists a $\sigma$-stable Borel subgroup in $H$.

Proof. (of Proposition A.2.)

Assume that $Z^{0}$ is not commutative. Let $\mathfrak{z}$ denote its Lie algebra. We claim that there exists a nilpotent element $n \in \mathfrak{z}, n \neq 0$, such that $\operatorname{ad}_{F}(n)=c \cdot n$ for a scalar $c$.

Indeed, $\operatorname{ad}_{F}$ defines an automorphism of $Z^{0}$ (it is obvious that $Z$ and hence also $Z^{0}$ is normalized by $F$ ). Therefore, the above lemma shows that there exists a Borel subgroup $B_{Z^{0}} \subset Z^{0}$, which is normalized by $F$. Let $\mathfrak{b}_{Z^{0}}$ be its Lie algebra and let $\mathfrak{n}_{Z^{0}} \subset \mathfrak{b}_{Z^{0}}$ be the nilpotent radical of this Lie algebra. Then $\mathfrak{n}_{Z^{0}}$ is also normalized by $F$. We choose $n \in \mathfrak{n}_{Z^{0}}$ to be an eigenvector of $\operatorname{ad}_{F}$.

Thus, let $n \in \mathfrak{z}$ be as above. It is well-known that to every nilpotent element $n$ in $\mathfrak{\mathfrak { g }}$ one can canonically associate a parabolic subgroup $\check{P}_{n}$ in $\check{G}$. The subgroup $\check{P}_{n}$ can be uniquely characterized in the following way:

Let $\check{\mathfrak{p}}_{n} \subset \check{\mathfrak{g}}$ be the Lie algebra of $\check{P}_{n}$. Fix a homomorphism $p: \operatorname{sl}(2) \rightarrow \check{\mathfrak{g}}$ in such a way that $p\left(\begin{array}{ll}0 & 1 \\ 0 & 0\end{array}\right)=n$. Let $\check{\mathfrak{g}}=\bigoplus_{i \in \mathbb{Z}} \check{\mathfrak{g}}_{i}$ be the weight decomposition of $\check{\mathfrak{g}}$ with respect to the adjoint action of $p(\operatorname{sl}(2))$. Then $\check{\mathfrak{p}}_{n}=\bigoplus_{i \geq 0} \check{\mathfrak{g}}_{i}$.

On the one hand, the fact that $n \neq 0$ implies that $\check{P}_{n} \neq \check{G}$. On the other hand, since $n$ is an eigenvector of $\widetilde{\Gamma}$ and the assignment $n \mapsto \check{P}_{n}$ is canonical, the group $\check{P}_{n}$ is also normalized by $\widetilde{\Gamma}$. But as $\check{P}_{n}$ is a parabolic subgroup in $\check{G}$, it follows that $\widetilde{\Gamma} \subset \check{P}_{n}$, which is a contradiction.

\section{REFERENCES}

[1] A. Beauville, Y. Laszlo, Un lemme de descente, C.R. Acad. Sci. Paris, Sér. I Math. 320 (1995), pp. 335-340.

[2] A. Beilinson, J. Bernstein and P. Deligne, Faisceaux Pervers, Astérisque 100 (1982), pp. 5-171.

[3] A. Beilinson and V. Drinfeld, Quantization of Hitchin's integrable system and Hecke eigensheaves, preprint.

[4] J. Bernstein, V. Lunts, Equivariant sheaves and functors, Lecture notes in mathematics 1578, Springer-Verlag, Berlin (1994).

[5] A. Braverman, D. Gaitsgory, Crystals via the affine Grassmannian, alg-geom/9909077, to appear in Duke Math. Jour.

[6] A. Braverman and D. Kazhdan, On the Schwartz space of the basic affine space, alg-geom/9809112. 
[7] A. Braverman, M. Finkelberg, D. Gaitsgory and I. Mirkovic Stalks of IC-sheaves on Drinfeld's spaces, forthcoming.

[8] P. Deligne, Théorèmes de finitude en cohomologie $\ell$-adique, SGA $4 \frac{1}{2}$, Lecture notes in mathematics 569, Springer-Verlag, Berlin-New York (1977).

[9] P. Deligne, La conjecture de Weil-II, Publications IHES 52 (1980), pp. 137-252.

[10] V. Drinfeld, C. Simpson B-structures on $G$-bundles and local triviality, Math. Res. Lett 2 (1995), pp. 823-829.

[11] M. Finkelberg and I. Mirkovic, Semi-infinite flags-I, alg-geom/9707010.

[12] B. Feigin, M. Finkelberg, A. Kuznetsov and I. Mirkovic, Semi-infinite flags-II, alg-geom/9711009.

[13] E. Frenkel, D. Gaitsgory and K. Vilonen, Whittaker patterns in the geometry of moduli spaces of bundles on curves, alg-geom/9907133.

[14] D. Gaitsgory, Automorphic sheaves and Eisenstein series, PhD Thesis (1997).

[15] D. Gaitsgory, Central elements in the affine Heckle algebra via nearby cycles, alg-geom/9912074.

[16] V. Ginzburg, Perverse sheaves on the loop group and Langlands' duality, preprint (1995).

[17] F. D. Grosshans, The invariants of unipotent radicals of parabolic subgroups, Invent. Math. 73 (1983), no. 1, 1-9.

[18] A. Kuznetsov, Laumon's resolution of Drinfeld's compactification is small, MRL 4 (1997), pp. 349-364.

[19] G. Laumon, Faisceaux automorphes liés aux séries d'Eisenstein, In: Automorphic forms, Shimura varieties and L-functions, Perspect. Math. 10, Academic Press, Boston, 1990.

[20] G. Lusztig, Singularities, character formulas and q-analog of weight multiplicities, Astérisque 101102 (1983), pp. 208-229.

[21] I. Mirković, K. Vilonen, Perverse sheaves on loop Grassmannians and Langlands duality, alggeom/9703010.

A.B.:Dept. of Math., Mit, Cambridge MA 02139; D.G: Dept. of Math., Harvard UniVERsity, CAMBridge MA 02138

E-mail address: braval@math.mit.edu, gaitsgde@math.harvard.edu 